NUREG-0020

Vol. 19

\title{
Licensed Operating Reactors
}

Status Summary Report

Data as of 12-31-94

Manuscript Completed: March 1995

Date Published: April 1995

Office of Information Resources Management

U.S. Nuclear Regulatory Commission

Washington, DC 20555-0001 


\section{DISCLAIMER}

This report was prepared as an account of work sponsored by an agency of the United States Government. Neither the United States Government nor any agency thereof, nor any of their employees, make any warranty, express or implied, or assumes any legal liability or responsibility for the accuracy, completeness, or usefulness of any information, apparatus, product, or process disclosed, or represents that its use would not infringe privately owned rights. Reference herein to any specific commercial product, process, or service by trade name, trademark, manufacturer, or otherwise does not necessarily constitute or imply its endorsement, recommendation, or favoring by the United States Government or any agency thereof. The views and opinions of authors expressed herein do not necessarily state or reflect those of the United States Government or any agency thereof. 


\section{DISCLAIMER}

Portions of this document may be illegible in electronic image products. Images are produced from the best available original document. 
The Nuclear Regulatory Commission's annual summary of licensed nuclear power reactor data is based primarily on the report of operating data submitted by licensees for each unit for the month of December because that report contains data for the month of December, the year to date (in this case calendar year 1994) and cumulative data, usually from the date of commercial operation. The data is not independently verified, but various computer checks are made.

The report is divided into two sections. The first contains summary highlights and the second contains data on each individual unit in commercial operation.

Section 1 capacity and availability factors are simple arithmetic averages. Section 2 items in the cumulative column are generaliy as reported by the licensee and notes as to the use of weighted averages and starting dates other than commercial operation are provided. 



\section{TABLE OF CONTENTS}

ABSTRACT

INTRODUCTION

vii

ACKNOWLEDGEMENT

ix

GLOSSARY

$x i$

SECTION 1 CURRENT DATA SUMMARIES

$1-1$

SECTION 2 OPERATING POWER REACTORS

ARKANSAS 1 through ZION 2

2-1 through 2-327

$-\mathrm{V}-$ 


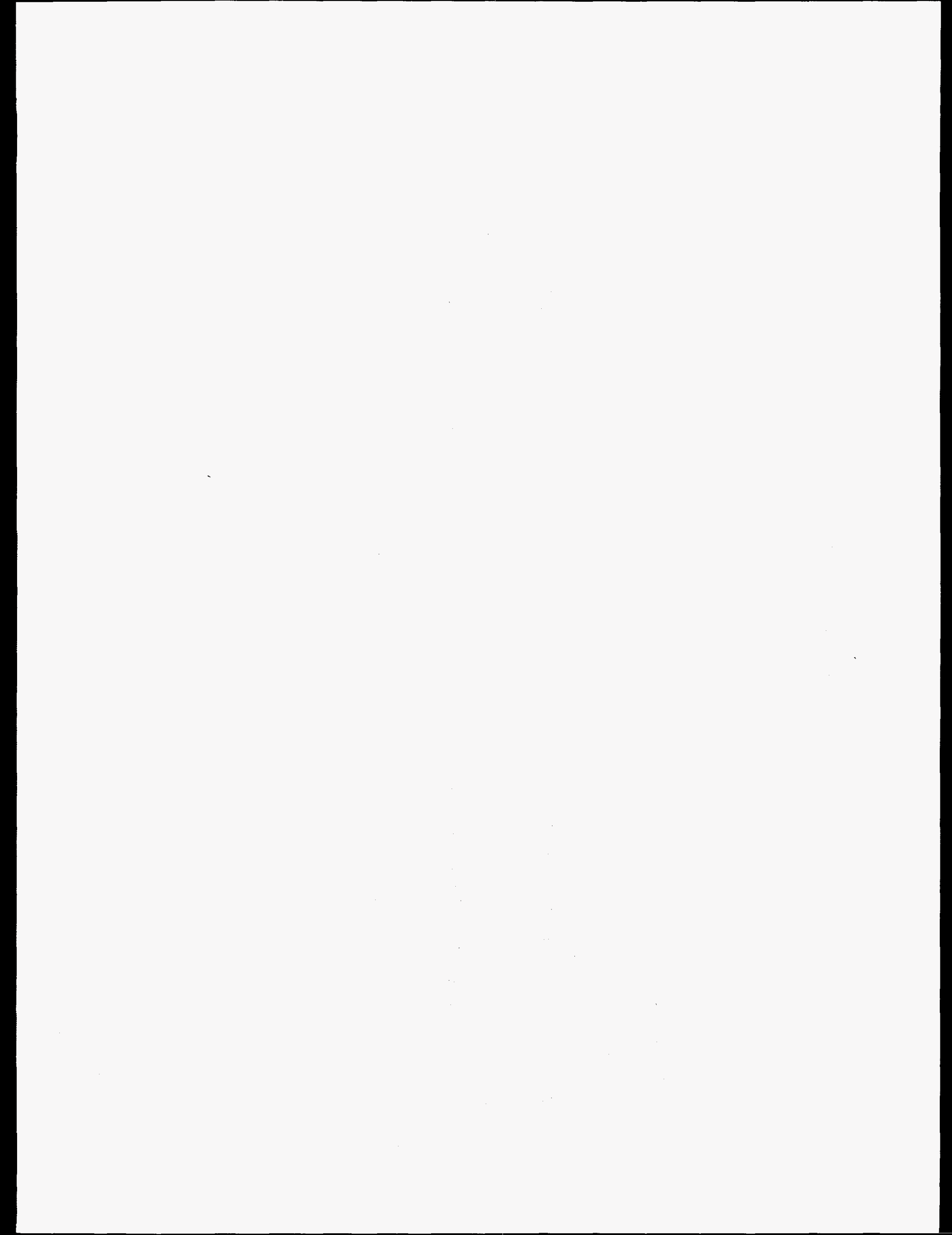




\section{INTRODUCTION}

The document titled "Licensed Operating Reactors - Status Summary Report" was issued on a monthly basis by the Nuclear Regulatory Commission since the mid-seventies and was commonly called the "Gray Book". The last monthly publication was issued as NUREG-0020, Vol.14, No. 3, including data as of February 28, 1990. A questionnaire/survey was enclosed in that publication to assess interest in the data in electronic format. A majority of respondents indicated interest in the electronic format, but many other users suggested an annual publication be printed that would contain a summary for a calendar year, similar to the January issue of the old Gray Book. This report, NUREG-0020, Vol. 19 is the fifth report of the new annual publication.

This report will not contain all of the information formerly contained in the Gray Book, but will contain the data the survey determined was essential to most users. In addition to this report, diskettes containing the same type of information in electronic form will be prepared by NRC and will be available for sale as a subscription from the Government Printing office.

For calendar year 1990 data a package of 12 diskettes is available from GPO as a single sales item. Future annual reports may be purchased from the Superintendent of Documents, U.S. Government Printing 0ffice, P.0.Box 37082, Washington, D.C., 20013-7082. 



\section{ACKNOWLEDGEMENT}

The work of maintaining the data bases, data quality assurance and report generation was performed by $L$. $L$. Brown, G. D. Roberts, T. W. Smith and C. D. White of the Idaho National Engineering Laboratory. 


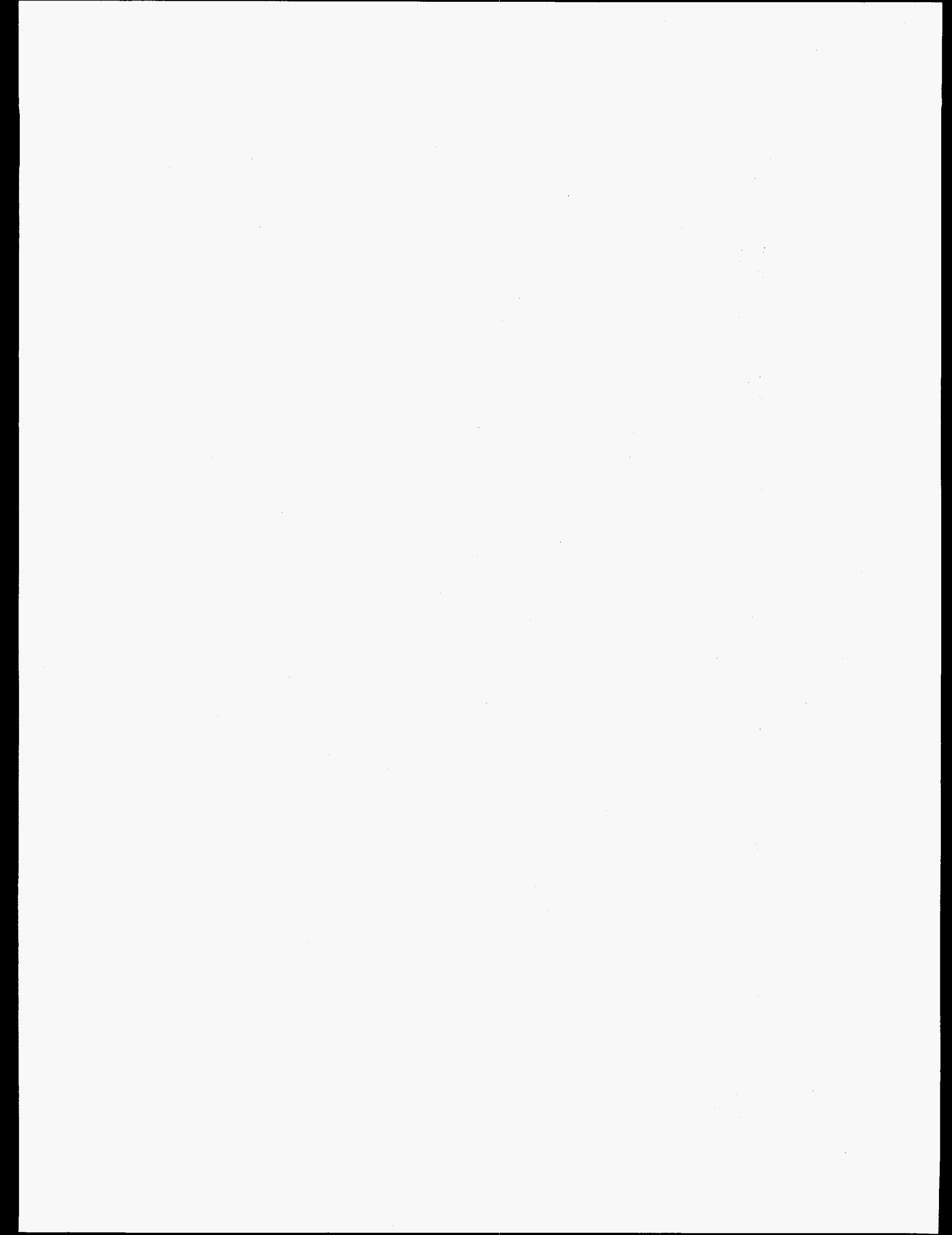




\section{GLOSSARY}

AVERAGE DAILY POWER LEVEL (MWO)

LICENSED THERMAL POWER (MWt)

DATE OF COMMERCIAL OPERATION

DESIGN ELECTRICAL RATING (DER) (NET MWe)

FORCED OUTAGE

FORCED OUTAGE HOURS

GROSS ELECTRICAL ENERGY GENERATED (MWH)

GROSS HOURS

GROSS THERMAL ENERGY GENERATED (MWH)

HOURS GENERATOR ON-LINE
The net electrical energy generated during the day (measured from 0001 to 2400 hours inclusivel in megawatts hours, divided by 24 hours.

The maximum thermal power of the reactor authorized by the NRC, expressed in megawatts.

Date unit was declared by utility owner to be available for the regular production of electricity; usually related to satisfactory completion of qualification tests as specified in the purchase contract and to accounting policies and practices of utility.

The nominal net electrical output of the unit specified by the utility and used for the purpose of plant design.

An outage required to be initiated no later than the weekend following discovery of an offnormal condition.

The clock hours during the report period that a unit is unavailable due to forced outages.

Electrical output of the unit during the report period as measured at the output terminals of the turbine generator, in megawatt hours.

The clock hours from the beginning of a specified situation until its end. For outage durations, the clock hours during which the unit is not in power production.

The thermal energy produced by the unit during the report period as measured or computed by the licensee in megawatt hours.

Also, "Unit Service Hours." The total clock hours in the report period during which the unit operated with breakers closed to the station bus. These hours added to the total outage hours experienced by the unit during the report period, shall equal the hours in the report period. 


\section{GLOSSARY (Continued)}

HOURS IN REPORTING PERIOD

\section{HOURS REACTOR CRITICAL}

MAXIMUM DEPENDABLE CAPACITY GROSS (MDC Gross) (Gross MWe)

MAXIMUM DEPENDABLE CAPACITY NET (MDC Net) (Net MWe)

NAMEPLATE RATING (GTOSS MNWE)

NET ELECTRICAL ENERGY GENERATED

OUTAGE

OUTAGE DATE
For units in power ascension at the end of the period, the gross hours from the beginning of the period or the first electrical production, whichever comes last, to the end of the period.

For units in commercial operation at the end of the period, the gross hours from the beginning of the period or of commercial operation, whichever comes last, to the end of the period or decommissioning, whichever comes first.

The total clock hours in the report period during which the reactor sustained a controlled chain reaction.

Dependable main-unit gross capacity, winter or summer, whichever is smaller. The dependable capacity varies because the unit efficiency varies during the year due to cooling water temperature variations. It is the gross electrical output as measured at the output terminals of the turbine generator during the most restrictive seasonal conditions (usually summer).

Maximum Dependable Capacity Gross less the normal station service loads.

The nameplate power designation of the generator in megavolt amperes (MVA) times the nameplate rating power factor of the generator. NOTE: The nameplate rating of the generator may not be indicative of the maximum dependable capacity, since some other item of equipment of a lesser rating (e.g., turbine) may limit unit output.

Gross electrical output of the unit measured at the output terminals of the turbine generator during the reporting period, minus the normal station service electrical energy utilization. If this quantity is less than zero, a negative number should be recorded.

A situation in which no electrical production takes place.

As reported on Appendix D of Reg. Guide 1.16, the date of the start of the outage. If continued from a previous month, report the same outage date but change "Method of Shutting Down Reactor" to " 4 (continuations)" and add a note: "Continuation from previous month." 


\section{GLOSSARY (Continued)}

OUTAGE DURATION

OUTAGE NUMBER

PERIOD HOURS

POWER REDUCTION

REACTOR AVAILABLE HOURS

REACTOR AVAILABILITY FACTOR

REACTOR RESERVE SHUTDOWN

REACTOR RESERVE SHUTDOWN HOURS

REACTOR SERVICE FACTOR

REPORT PERIOD
The total clock hours of the outage measured from the beginning of the report period or the outage, whichever comes last, to the end of the report period or the outage, whichever comes first.

A number unique to the outage assigned by the licensee. The same number is reported each month in which the outage is in progress. One format is "76-05" for the fifth outage to occur in 1976.

See "Hours in Reporting Period."

A reduction in the Average Daily Power Level of more than $20 \%$ from the previous day. All power reductions are defined as outages of zero hours duration for the purpose of computing unit service and availability factors, and forced outage rate.

The total clock hours in the report period during which the reactor was critical or was capable of being made critical. (Reactor Reserve Shutdown Hours + Hours Reactor Critical.)

\section{Reactor Available Hours $\times 100$ \\ Period Hours}

The cessation of criticality in the reactor for administrative or other similar reasons when operation could have been continued.

The total clock hours in the report period that the reactor is in reserve shutdown mode. NOTE: No credit is given for NRC imposed shutdowns.

Hours Reactor Critical $\times 100$

$$
\text { Period Hours }
$$

Usually, the preceding calendar month. Can also be the preceding calendar year lyearto-date), or the life-span of a unit (cumulative). 


\section{GLOSSARY (Continued)}

RESTRICTED POWER LEVEL

SCHEDULED OUTAGE

STARTUP AND POWER ASCENSION TEST PHASE

UNIT

UNIT AVAILABLE HOURS

UNIT AVAILABILITY FACTOR

UNIT CAPACITY FACTORS

- Using Licensed Thermal Power

- Using Nameplate Rating

- Using DER

- Using MDC Gross
Maximum net electrical generation to which the unit is restricted during the report period due to the state of equipment, external conditions, administrative reasons, or a direction by NRC.

Planned removal of a unit from service for refueling, inspection, training, or maintenance. Those outages which do not fit the definition of "Forced Outage" perforce are "Scheduled Outages."

Period following initial criticality during which the unit is tested at successively higher levels, culminating with operation at full power for a sustained period and completion of warranty runs. Following this phase, the utility generally considers the unit to be available for commercial operation.

The set of equipment uniquely associated with the reactor, including turbine generators and ancillary equipment, considered as a single electrical energy production facility.

The total clock hours in the report period during which the unit operated on-line or was capable of such operation. (Unit Reserve Shutdown Hours + Hours Generator OnLine.)

Unit Available Hours $\times 100$

Period Hours

Gross Thermal Eneray Generated $\times 100$

Period Hours $x$ Lic. Thermal Power

Gross Electrical Eneray Generated $\times 100$

Period Hours $\times$ Nameplate Rating

Net Electrical Eneroy Generated x 100

Period Hours $\times$ DER

Gross Electrical Enerov Generated $\times 100$

Period Hours x MDC Gross 


\section{GLOSSARY (Continued)}

- Using MDC Net

\section{Net Electrical Eneray Generated x 100}

Period Hours $x$ MDC Net

NOTE: If MDC Gross and/or MDC Net have not been determined, the DER Net is substituted for this quantity for Unit Capacity Factor calculations.

UNIT FORCED OUTAGE RATE

UNIT RESERVE SHUTDOWN

UNIT RESERVE SHUTDOWN HOURS

UNIT SERVICE FACTOR

UNIT SERVICE HOURS
Forced Outage Hours $\times 100$

Unit Service Hours + Forced Outage Hours

The removal of the unit from on-line operation for economic or other similar reasons when operation could have been continued.

The total clock hours in the report period during which the unit was in reserve shutdown mode.

\section{Unit Service Hours $\times 100$}

Period Hours

See "Hours Generator On-Line." 


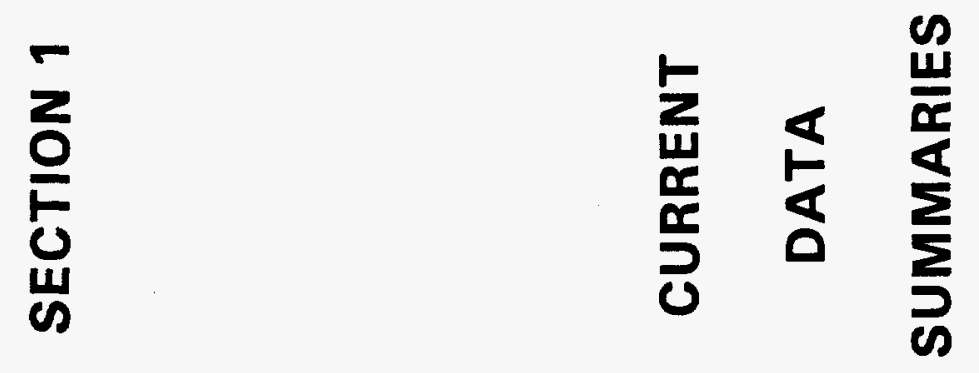




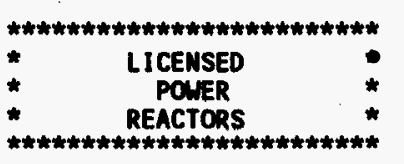

MOC Net
107 IN COMMERCIAL OPERATION. . . . . .
IN POUER ASCENSION .........
$\overline{107}$ LICENSED TO OPERATE. . . . . .
LICENSED FOR FUEL LOADING AND LOW PONER TESTING

(a)

(b) Excludes these plants licensed for operation wich are shut down indefinitely or permanently.

96,753

$\overline{96,753}$ TOTAL

\section{Net}

(c)
DATE

DER Net

$\begin{array}{lr}\text { 1. BRONNS FERRY } 1 & 0 \\ \text { 2. BROWNS FERRY } 3 & 0 \\ \text { 3. FORT ST VRAIN } & 330 \\ \text { 4. RANCHO SECO } & 873 \\ \text { 5. SAN ONOFRE 1 } & 436 \\ \text { 6. TROJAN } & 1095 \\ \text { 7. YANKEE-ROWE } & 167\end{array}$
CAPACITY we (Net) -- Based upon maximum dependable capacity (MDC Net); design electrical rating (DER Net) used if MOC Net not determined.

REPORT MONTH

$63,487,930.0$

$60,694,180.7$

86.9

86.9

84.7

82.7

6.7
YEAR TO DATE

$672,392,448.8$

$641,725,706.5$

79.1

79.1

76.4

74.6

9.0
Note: Values for $i$ tems 1 and 2 were calculated using data from all licensed reactors producing electrical power. Values for items 3 through 7 were calculated using data from only those reactors in commercial operation. Values for item 5 were calculated using DER Net if MOC Net was not determined. 


$$
\begin{aligned}
& N \\
& 2 \\
& 0 \\
& \omega
\end{aligned}
$$

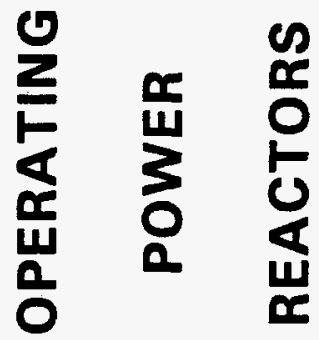


1. Docket: $50-313$

OPERATING STATUS

2. Reporting Period: DECEMBER 1994

Outage + On-Line Hrs: 744.0

3. Utility Contact: K. R. HAYES (501) 858-5535

4. Licensed Thermal Power (MUt):

5. Nameplate Rating (Gross Mue):

6. Design Electrical Rating (Net MWe):

7. Maximm Dependable Capecity (Gross MHe):

8. Maximum Dependable Capacity (Met MHe):

9. If Changes Occurred Above Since Last Report, Give Reasons:

10. Power Level To Which Restricted, If Any (Net MWe):

11. Reasons For Restrictions, If Any:

\begin{tabular}{|c|c|c|c|}
\hline & MONTH & YEAR & CUMULATIVE \\
\hline 12. Report Period Hrs & 744.0 & $8,760.0$ & $175,627.0$ \\
\hline 13. Hours Reactor Critical & 744.0 & $8,657.8$ & $129,256.2$ \\
\hline 14. Rx Reserve Shtdwn Hrs & 0.0 & 0.0 & $5,044.0$ \\
\hline 15. Hrs Generator on-Line & 744.0 & $8,644.4$ & $126,988.0$ \\
\hline 16. Unit Reserve Shtdin Hrs & 0.0 & 0.0 & 817.5 \\
\hline 17. Gross Therm Ener (MUH) & $1,891,208.5$ & $22,103,326.2$ & $294,153,895.2$ \\
\hline 18. Gross Elec Ener (MUH) & $646,780.0$ & $7,518,885.0$ & $98,311,920.0$ \\
\hline 19. Net Elec Ener (MWH) & $620,116.0$ & $7,198,561.0$ & $93,525,697.0$ \\
\hline 20. Unit Service Factor & 100.0 & 98.7 & 72.3 \\
\hline 21. Unit Avail Factor & 100.0 & 98.7 & 72.8 \\
\hline 22. Unit Cap Factor (MDC Net) & 99.7 & 98.3 & 63.7 \\
\hline 23. Unit Cap Factor (DER Net) & 98.1 & 96.7 & 62.7 \\
\hline 24. Unit Forced Outage Rate & 0.0 & 1.3 & 10.7 \\
\hline 25. Forced Outage Hours & 0.0 & 115.6 & $15,148.9$ \\
\hline
\end{tabular}

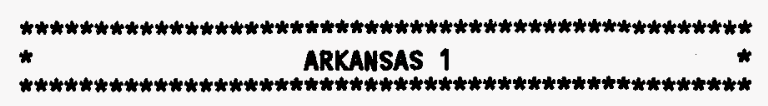

AVERAGE DAILY POWER LEVEL (Net MWE)

\begin{tabular}{|c|c|c|c|}
\hline DAY & PONER & DAY & POUER \\
\hline 1 & 841 & 16 & 843 \\
\hline 2 & 734 & 17 & 843 \\
\hline 3 & 691 & 18 & 843 \\
\hline 4 & 839 & 19 & 842 \\
\hline 5 & 841 & 20 & 843 \\
\hline 6 & 833 & 21 & 836 \\
\hline 7 & 837 & 22 & 839 \\
\hline 8 & 843 & 23 & 843 \\
\hline 9 & 843 & 24 & 842 \\
\hline 10 & 843 & 25 & 843 \\
\hline 11 & 843 & 26 & 841 \\
\hline 12 & 842 & 27 & 846 \\
\hline 13 & 843 & 28 & 845 \\
\hline 14 & 844 & 29 & 843 \\
\hline \multirow[t]{2}{*}{15} & 843 & 30 & 843 \\
\hline & & 31 & 844 \\
\hline
\end{tabular}

26. Shutdowns Scheduled Over Mext Six Months (Type, Date, Duration): REFUELING OUTAGE, februarY 14, 1995.

27. If Currently Shutdown, Estimated Startup Date:

Notes: 


\begin{tabular}{|c|c|c|c|c|c|c|c|c|c|}
\hline No. & Date & Type & Hours & Reason & Method & LER Number & System & Component & Cause and Corrective Action To Prevent Recurrence \\
\hline & $12 / 02 / 94$ & s & 0.0 & B & 5 & & & & $\begin{array}{l}\text { PONER REDUCTION FOR MAINTENANCE ON BOTH MAIN } \\
\text { FEEDWATER PUMPS AND E3A FEEDWATER HEATER: }\end{array}$ \\
\hline
\end{tabular}

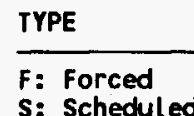

\section{REASON}

A-Equipment Fai Lure B-Maintenance or Test

D-Regulatory Restriction

E-Operator Training \&

License Examination

F-Administrative

G-Operational Error

H-other

\begin{tabular}{l} 
METHOD \\
\hline 1-Manual \\
2-Manual Scram \\
3-Auto Scram \\
4-Cont inued \\
5-Reduced Load \\
9-0ther
\end{tabular}

SYSTEM

IEEE Standard

805-1984 and/or
NUREG-0161 Exhibit F

\section{COMPONENT}

IEEE Standard

803A-1983 and/or

NUREG-0161 Exhibit H 


\section{FACILITY DESCRIPTION}

LOCATION

STATE

COUNTY.

ARKANSAS

POPE

\section{DIST AND DIRECTION FROM}

MEAREST POPULATION CTR..... 6 MI WWH OF RUSSELLVILLE, AR

TYPE OF REACTOR.

PUR

DATE INITIAL CRITICALITY...... AUGUST 06, 1974

DATE INITIAL ELECTRICITY...... AUGUST 17, 1974

DATE COMMERCIAL OPERATE....... DECEMBER 19, 1974

CONDENSER COOLING METHOD...... OHCE THRU

CONDENSER COOLING WATER....... DARDANELLE RESERVOIR

ELECTRIC RELIABILITY

COUNCIL................... SOUTHUEST PONER POOL
UTILITY \& CONTRACTOR INFORMATION

UTILITY

LICENSEE............... ENTERGY OPERATIONS, INC.

CORPORATE ADDRESS

ROUTE 3 BOX $137 \mathrm{G}$

RUSSEVILLE, ARKANSAS 72801

\section{CONTRACTOR}

ARCHITECT/ENGINEER.......... BECHTEL

NUC STEAM SYS SUPPLIER...... BABCOCK \& WILCOX

CONSTRUCTOR............. BECHTEL

TURBINE SUPPLIER........... MESTINGHOUSE

\section{REGULATORY INFORMATION}

IE REGION RESPONSIBLE........ 4

IE RESIDENT INSPECTOR......... KRISS KENNEDY

LICENSING PROJ MANAGER......... GEORGE KALMAN

DOCKET MUMBER............. $50-313$

LICENSE \& DATE ISSUANCE........ DPR 051, MAY 21, 1974 
1. Docket: $50-368$

DPERATING STATUS

2. Reporting Period: DECEMBER 1994 Outage + On-Line Hrs: $\mathbf{7 4 4 . 0}$

3. Utility Contact: M. S. HHITT (501) 858-5560

4. Licensed Thermal Power (MNt):

5. Nameplate Rating (Gross MWe):

6. Design Electrical Rating (Net MWe):

7. Maximum Dependable Capacity (Gross MWe):

8. Maximum Dependable Capacity (Net MWe):

9. If Changes Occurred Above Since Last Report, Give Reasons:

10. Power Level To Which Restricted, If Any (Net Wwe):

11. Reasons For Restrictions, If Any:

\begin{tabular}{|c|c|c|c|}
\hline & MONTH & YEAR & CUMULATIVE \\
\hline 12. Report Period Hrs & 744.0 & $8,760.0$ & $129,456.0$ \\
\hline 3. Hours Reactor Critical & 744.0 & $7,739.7$ & $100,536.2$ \\
\hline R. Rx Réserve Shtodun hrs & $\hat{0.0}$ & $\hat{0.00}$ & $\overline{0} . \overline{0}$ \\
\hline 5. Hrs Generator On-Line & 744.0 & $7,707.2$ & $98,633.1$ \\
\hline 6. Unit Reserve Shtdwn Hrs & 0.0 & 0.0 & 0.0 \\
\hline 17. Gross Therm Ener (MWH) & $2,078,706.0$ & $21,362,226.0$ & $262,342,209.3$ \\
\hline 18. Gross Elec Ener (МUH) & $699,534.0$ & $7,047,235.0$ & $86,369,572.0$ \\
\hline 19. Het Elec Ener (MUH) & $669,350.0$ & $6,724,879.0$ & $82,197,718.0$ \\
\hline . Unit Service Factor & 100.0 & 88.0 & 76.2 \\
\hline 1. Unit Avail Factor & 100.0 & 88.0 & 76.2 \\
\hline . Unit Cap Factor (MDC Net) & 104.9 & 89.5 & 74.0 \\
\hline Unit Cap Factor (DER Net) & 98.6 & 84.2 & 69.6 \\
\hline - Unit Forced Outage Rate & 0.0 & 0.0 & 10.5 \\
\hline Forced Outage Hours & 0.0 & 0.0 & $11,537.0$ \\
\hline
\end{tabular}

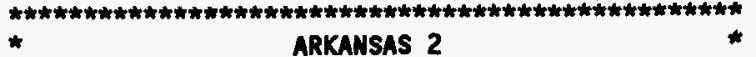

ARKANSAS 2

AVERAGe DAILY POWER LeVEL (Net MWe)

\begin{tabular}{ll} 
DAY & POWER \\
\hline 1 & 895 \\
\hline 2 & 896 \\
\hline 3 & 894 \\
\hline 4 & 894 \\
\hline 5 & 893 \\
\hline 6 & 894 \\
\hline 7 & 897 \\
\hline 8 & 908 \\
\hline 9 & 906 \\
\hline 10 & 909 \\
\hline 11 & 909 \\
\hline 12 & 910 \\
\hline 13 & 907 \\
\hline 14 & 901 \\
\hline 15 & 898 \\
\hline
\end{tabular}

\begin{tabular}{|c|c}
\hline DAY & PONER \\
\hline 16 & 897 \\
\hline 17 & 899 \\
\hline 18 & 899 \\
\hline 19 & 899 \\
\hline 20 & 898 \\
\hline 21 & 899 \\
\hline 22 & 899 \\
\hline 23 & 899 \\
\hline 24 & 899 \\
\hline 25 & 899 \\
\hline 26 & 899 \\
\hline 27 & 899 \\
\hline 28 & 898 \\
\hline 29 & 899 \\
\hline 30 & 899 \\
\hline 31 & 898 \\
\hline
\end{tabular}

26. Shutdowns Scheduled Over Mext six Months (Type, Date, Duration): MID-CYCLE STEAM GENERATOR IMSPECTION, JANUARY 7, 1995, 12 DAYS. 27. If Currently Shutdown, Estimated Startup Date: Notes: 


No. Date Type Hours Reason Method LER Number System Component Cause and Corrective Action To Prevent Recurrence

TYPE

F: Forced

s: Scheduled

\begin{tabular}{l} 
REASON \\
\hline A-Equipment Failure \\
B-Maintenance or Test \\
C-Refuel ing \\
D-Regulatory Restriction \\
E-Operator Training \& \\
License Examination \\
F-Administrative \\
G-Operational Error
\end{tabular}

\section{METHOD}

1-Manual

2-Manual Scran

3-Auto Scram

4-Cont inued

5-Reduced Load

9-other

Adminis

G-Operational Error

\section{SYSTEM \\ IEEE Standard \\ 805-1984 and/or}

MUREG-0161 Exhibit $F$

\section{COMPONENT}

IEEE Standard

NUREG-0161 Exhibit H 


\section{FACILITY DESCRIPTION}

\section{LOCATION}

STATE................. ARKANSAS

DIST AND DIRECTION FRO

TYPE OF REACTOR.

PUR

DATE INITIAL CRITICALITY...... DECEMBER 05, 1978

DATE INITIAL ELECTRICITY...... DECEMBER 26, 1978

DATE COMMERCIAL OPERATE........ MARCH 26, 1980

CONDENSER COOLING METHOO..... COOLING TONER

CONDENSER COOLING MATER....... DARDANELLE RESERVOIR

ELECTRIC RELIABILITY

COUNCIL................... SOUTHWEST POWER POOL
UTILITY \& CONTRACTOR INFORMATION

\section{UTILITY}

LICENSEE................. ENTERGY OPERATIONS, INC.

CORPORATE ADDRESS

\section{ROUTE 3 BOX 137G}

RUSSEVILLE, ARKANSAS 72801

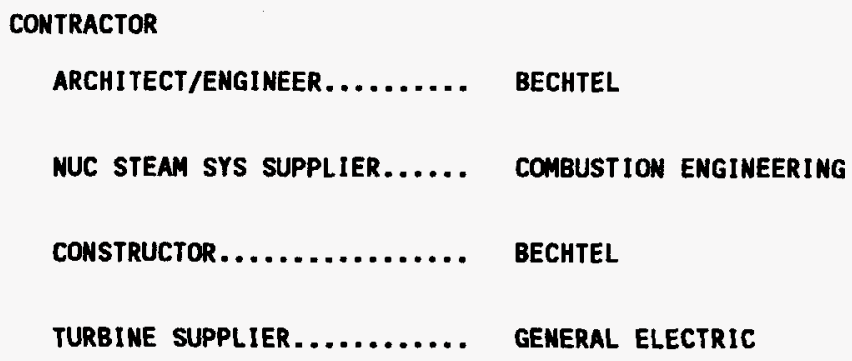

IE REGION RESPONSIBLE:....... 4

IE RESIDENT INSPECTOR

KRISS KENMEDY

LICENSING PROJ MANAGER.

GEORGE KALMAN

DOCKET NUMBER

$50-368$

LICENSE \& DATE ISSUANCE.

NPF 006, SEPTEMBER 01, 1978 
1. Docket: $50-334$

OPERATING STATUS

2. Reporting Period: DECEMBER 1994

Outage + On-Line Hrs:

744.0

3. Utility Contact: DAVID T. JONES (412) 393-7553

4. Licensed Thermal Power (MUt):

5. Nameplate Rating (Gross MHe):

6. Design Electrical Rating (Net MWe):

7. Maximum Dependable Capacity (Gross Me):

8. Maximum Dependable Capacity (Net MHe):

\begin{tabular}{c}
\hline 2652 \\
\hline 923 \\
\hline 835 \\
\hline 860 \\
\hline 810
\end{tabular}

9. If Changes Occurred Above since Last Report, Give Reasons:

10. Power Level To thich Restricted, If Any (Wet MWe):

11. Reasons For Restrictions, If Any:

\begin{tabular}{|c|c|c|c|}
\hline 12. Report Period Hrs & $\begin{array}{l}\text { MONTH } \\
744.0\end{array}$ & $\begin{array}{l}\text { YEAR } \\
8,760.0\end{array}$ & $\begin{array}{l}\text { CUMULATIVE } \\
163,656.0\end{array}$ \\
\hline 3. Hours Reactor Critical & 744.0 & $7,025.9$ & $106,556.8$ \\
\hline 14. Rx Reserve Shtdwn Hrs & 0.0 & 0.0 & $4,482.8$ \\
\hline 15. Hrs Generator On-Line & 744.0 & $6,994.5$ & $104,537.7$ \\
\hline 6. Unit Reserve Shtdwn Hrs & 0.0 & 0.0 & 0.0 \\
\hline 17. Gross Therm Ener (MWH) & $1,724,487.0$ & $17,921,590.0$ & $251,327,066.5$ \\
\hline 18. Gross Elec Ener (MHH) & $568,210.0$ & $5,868,444.0$ & $81,025,967.0$ \\
\hline 19. Net Elec Ener (MWH) & $534,480.0$ & $5,504,384.0$ & $75,723,904.0$ \\
\hline 20. Unit Service Factor & 100.0 & 79.8 & 65.8 \\
\hline 1. Unit Avail Factor & 100.0 & 79.8 & 65.8 \\
\hline 22. Unit Cap Factor (MDC Net) & 88.7 & 77.6 & 59.6 \\
\hline 23. Unit Cap Factor (DER Net) & 86.0 & 75.3 & 57.8 \\
\hline 4. Unit Forced Outage Rate & 0.0 & 20.2 & 16.1 \\
\hline Forced Outage Hours & 0.0 & 1765.5 & $19,477.5$ \\
\hline
\end{tabular}

***************************************************

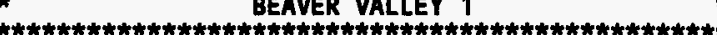

AVERAGe DAILY POHER LeVel (Net MHe)

\begin{tabular}{ll} 
DAY & POWER \\
\hline 1 & 833 \\
\hline 2 & 825 \\
\hline 3 & 821 \\
\hline 4 & 821 \\
\hline 5 & 825 \\
\hline 6 & 825 \\
\hline 7 & 825 \\
\hline 8 & 830 \\
\hline 9 & 829 \\
\hline 10 & 829 \\
\hline 11 & 775 \\
\hline 12 & 754 \\
\hline 13 & 775 \\
\hline 14 & 813 \\
\hline 15 & 792 \\
\hline
\end{tabular}

\begin{tabular}{ll} 
DAY & PONER \\
\hline 16 & 796 \\
\hline 17 & 775 \\
\hline 18 & 775 \\
\hline 19 & 779 \\
\hline 20 & 750 \\
\hline 21 & 754 \\
\hline 22 & 742 \\
\hline 23 & 754 \\
\hline 24 & 700 \\
\hline 25 & 492 \\
\hline 26 & 458 \\
\hline 27 & 475 \\
\hline 28 & 458 \\
\hline 29 & 462 \\
\hline 30 & 467 \\
\hline 31 & 463 \\
\hline
\end{tabular}

26. Shutdowns Scheduled Over Mext six Months (Type, Date, Duration): REFUELING OUTAGE, JANUARY 2, 1995, 60 DAYS.

27. If Currently Shutdown, Estimated Startup Date: Notes:

CUMULATIVE VALUES FOR ITEMS $12,13,15$, AND 17-19 INCLUDE PRECOMMERCIAL DATA, WHILE CUMULATIVE VALUES FOR ITEMS 20-25 ARE

CALCULATED SINCÉ COMMERCIAL OPERATION. 
TYPE

F: Forced

S: Scheduled

\begin{tabular}{l} 
REASON \\
\hline A-Equipment Failure \\
B-Maintenance or Test \\
C-Refueling \\
D-Regulatory Restriction \\
E-Operator Iraining \& \\
License Examination \\
F-Adninistrative \\
G-Operational Error
\end{tabular}

METHOD

1-Manual 2-Manual Scram 3-Auto Scram

4-Cont inued

5-Reduced Load

9-other

\begin{tabular}{|c|c|}
\hline SYSTEM & COMPONENT \\
\hline $\begin{array}{l}\text { IEEE Standard } \\
805-1984 \text { and/or } \\
\text { MUREG-0161 Exhibit F }\end{array}$ & $\begin{array}{l}\text { IEEE Standard } \\
803 A-1983 \text { and/o } \\
\text { NUREG-0161 Exhi }\end{array}$ \\
\hline
\end{tabular}

MUREG-0161 Exhibit F MURE-0161 Exhibit 
FACILITY DESCRIPTION

LOCATION

STATE

COUNTY

PENNSYLVANIA

BEAVER

DIST AND DIRECTION FROM

TYPE OF REACTOR

DATE IMITIAL CRITICALITY...... MAY 10, 1976

DATE INITIAL ELECTRICITY...... JUNE 14, 1976

dATE COMMERCIAL OPERATE....... OCTOBER 01, 1976

CONDENSER COOLIMG METHOD...... COOLING TONER

CONDENSER COOLING MATER....... OHIO RIVER

ELECTRIC RELIABILITY

COUNCIL................ EAST CENTRAL AREA RELIABILITY

\section{UTILITY \& CONTRACTOR INFORMATION}

UTILITY

LICENSEE................. DUQUESNE LIGHT CO.

CORPORATE ADDRESS........... P.O. BOX

SHIPPINGPORT, PENMSYLVANIA 1507

\section{CONTRACTOR}

ARCHITECT/ENGINEER.

STONE \& WEBSTER

MUC STEAM SYS SUPPLIER ......

MESTINGHOUSE

CONSTRUCTOR

STONE \& WEBSTER

TURBINE SUPPLIER.

WESTIMGHOUSE

\section{REGULATORY IMFORMATION}

IE REgION RESPONSIBLE........ 1

IE RESIDENT INSPECTOR

LAWREMCE H. ROSSBACH

LICENSING PROJ MANAGER.

GORDON E. EDISON

DOCKET MUMBER.

$50-334$

LICENSE \& DATE ISSUANCE.

DPR 066, JULY 02, 1976 
1. Docket: $50-412$

OPERATING STATUS

2. Reporting Period: DECEMBER 1994 Outage + On-Line Hrs: $\mathbf{7 4 4 . 0}$

3. Utility Contact: DAVID T. JONES (412) 393-7553

4. Licensed Thermal Power (MHt):

5. Nameplate Rating (Gross MWe):

6. Design Electrical Rating (Net Mue):

7. Maximm Dependable Capacity (Gross MWe):

8. Maximum Dependable Capacity (Net MHe):

9. If Changes Occurred Above Since Last Report, Give Reasons:

10. Power Level To Which Restricted, If Any (Net MHe):

11. Reasons For Restrictions, If Any:

\begin{tabular}{|c|c|c|c|}
\hline & MONTH & YEAR & CUMULATIVE \\
\hline 12. Report Period Hrs & 744.0 & $8,760.0$ & $62,439.0$ \\
\hline 13. Hours Reactor Critical & 744.0 & $8,494.2$ & $53,824.5$ \\
\hline 14. RX Reserve Shtdwn Hrs & 0.0 & 0.0 & 0.0 \\
\hline 15. Hrs Generator On-Line & 744.0 & $8,481.0$ & $53,477.6$ \\
\hline 16. Unit Reserve Shtdun Hrs & 0.0 & 0.0 & 0.0 \\
\hline 17. Gross Therm Ener (MUH) & $1,961,570.0$ & $22,289,437.0$ & $132,521,322.4$ \\
\hline 18. Gross Elec Ener (MUH) & $653,239.0$ & $7,399,788.0$ & $43,051,793.0$ \\
\hline 19. Net Elec Ener (MUH) & $621,193.0$ & $7,024,726.0$ & $40,675,005.0$ \\
\hline 20. Unit Service Factor & 100.0 & 96.8 & 85.6 \\
\hline 21. Unit Avail Factor & 100.0 & 96.8 & 85.6 \\
\hline 22. Unit Cap Factor (MDC Net) & 101.8 & 97.8 & 79.0 \\
\hline 23. Unit Cap Factor (DER Net) & 99.9 & 95.9 & 77.9 \\
\hline 24. Unit Forced Outage Rate & 0.0 & 3.2 & 2.9 \\
\hline 25. Forced Outage Hours & 0.0 & 279.0 & $1,622.8$ \\
\hline
\end{tabular}

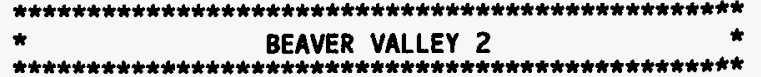

AVERAGE DAILY PONER LEVEL (Net MWe)

\begin{tabular}{ll} 
DAY & POWER \\
\hline & 835 \\
\hline 2 & 831 \\
\hline 3 & 830 \\
\hline 4 & 829 \\
\hline 5 & 825 \\
\hline 6 & 829 \\
\hline 7 & 832 \\
\hline 8 & 835 \\
\hline 9 & 831 \\
\hline 10 & 833 \\
\hline 11 & 832 \\
\hline 12 & 839 \\
\hline 13 & 837 \\
\hline 14 & 839 \\
\hline 15 & 835 \\
\hline
\end{tabular}

\begin{tabular}{ll} 
DAY & PONER \\
\hline 16 & 835 \\
\hline 17 & 835 \\
\hline 18 & 837 \\
\hline 19 & 838 \\
\hline 20 & 839 \\
\hline 21 & 838 \\
\hline 22 & 837 \\
\hline 23 & 836 \\
\hline 24 & 836 \\
\hline 25 & 837 \\
\hline 26 & 837 \\
\hline 27 & 840 \\
\hline 28 & 836 \\
\hline 29 & 838 \\
\hline 30 & 838 \\
\hline 31 & 834 \\
\hline
\end{tabular}

26. Shutdowns Scheduled Over Next six Months (Type, Date, Duration): REFUELING OUTAGE, MARCH 24, 1995, 60 DAYS.

27. If Currently Shutdoun, Estimated Startup Date:

Notes:

CUMULATIVE UNIT CAPACITY FACTOR (MDC NET) IS CALCULATED WITH A WEIGHTED AVERAGE. 


\begin{tabular}{|c|c|}
\hline TYPE & REASON \\
\hline $\begin{array}{l}\text { F: Forced } \\
\text { S: Scheduled }\end{array}$ & $\begin{array}{l}\text { A-Equipment Failure } \\
\text { B-Maintenance or Test } \\
\text { C-Refuel ing } \\
\text { D-Regulatory Restriction } \\
\text { E-Operator Iraining \& } \\
\text { License Examination } \\
\text { F-Administrative } \\
\text { G-Operat ional Error } \\
\text { H-Other }\end{array}$ \\
\hline
\end{tabular}

\begin{tabular}{l} 
METHOO \\
\hline 1-Manual \\
2-Manual Scram \\
3-Auto Scram \\
4-Continued \\
5-Reduced Load \\
9-Other
\end{tabular}

\section{SYSTEM}

IEEE Standard

NUREG-0161 Exhibit F

G-Operational Error

H-Other 


\section{FACILITY DESCRIPTION}

\section{LOCATION}

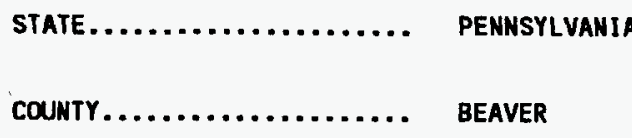

DIST AND DIRECTION FROM MEAREST POPULATION CTR..... 17 MI W OF MCCANDLESS, PA

TYPE OF REACTOR.

PWR

DATE INITIAL CRITICALITY.

DAATE INITIAL ELECTRICITY.

DATE COMMERCIAL OPERATE

CONDENSER COOLIMG METHOD

CONDENSER COOLING MATER

ELECTRIC RELIABILITY

COUNCIL................

\section{UTILITY \& CONTRACTOR INFORMATION}

UTILITY

LICENSEE DUQUESNE LIGHT CO.

CORPORATE ADDRESS

P.0. BOX 4 SHIPPINGPORT, PENNSYLVANIA 15077

\section{CONTRACTOR}

ARCHITECT/ENGINEER.

NUC STEAM SYS SUPPLIER.

CONSTRUCTOR

TURBINE SUPPLIER.

REGULATORY INFORMATION

IE REGION RESPONSIBLE........ 1

IE RESIDENT INSPECTOR.

LAURENCE W. ROSSBACH

LICENSING PROJ MANAGER.

GORDON E. EDISON

EAST CENTRAL AREA RELIAB
COORDINATION AGREEMENT 


\section{Docket: $50-155$}

OPERATING STATUS

2. Reporting Period: DECEMBER 1994

Outage + On-Line Hrs: 744.0

3. Utility Contact: J. R. JOHNSTON (616) 547-6537 EXT. 223

4. Licensed Thermal Power (MWt):

5. Nameplate Rating (Gross MWe):

6. Design Electrical Rating (Net MHe):

7. Maximum Dependable Capacity (Gross MHe):

8. Maximum Dependable Capacity (Net MWe):

9. If Changes Occurred Above Since Last Report, Give Reasons:

10. Power Level To Which Restricted, If Any (Net MWe):

11. Reasons For Restrictions, If Any:

\begin{tabular}{|c|c|c|c|}
\hline & MONTH & YEAR & CUMULATIVE \\
\hline 12. Report Period Hrs & 744.0 & $8,760.0$ & $278,419.0$ \\
\hline 13. Hours Reactor Critical & 744.0 & $6,598.9$ & $201,714.5$ \\
\hline 14. Rx Reserve Shtdun Hrs & 0.0 & 0.0 & 0.0 \\
\hline 15. Hrs Generator On-Line & 744.0 & $6,451.9$ & $198,531.1$ \\
\hline 16. Unit Reserve Shtdwn Hrs & 0.0 & 0.0 & 0.0 \\
\hline 17. Gross Therm Ener (MMH) & $141,868.0$ & $1,350,723.0$ & $38,253,714.0$ \\
\hline 18. Gross Elec Ener (MUH) & $45,796.0$ & $433,590.0$ & $12,155,705.0$ \\
\hline 19. Net Elec Ener (MWH) & $43,327.2$ & $410,472.7$ & $11,499,732.7$ \\
\hline 20. Unit Service Factor & 100.0 & 73.7 & 71.3 \\
\hline 21. Unit Avail Factor & 100.0 & 73.7 & 71.3 \\
\hline 22. Unit Cap Factor (MDC Net) & 86.9 & 69.9 & 61.4 \\
\hline 23. Unit Cap Factor (DER Net) & 80.9 & 65.1 & 57.4 \\
\hline 24. Unit Forced Outage Rate & 0.0 & 6.2 & 11.0 \\
\hline 25. Forced Outage Hours & 0.0 & 425.6 & $16,417.3$ \\
\hline
\end{tabular}

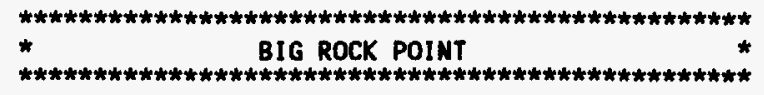

AVERAGE DAILY POWER LEVEL (Net MWe)

\begin{tabular}{lc} 
DAY & PONER \\
\hline 1 & 39 \\
\hline 2 & 44 \\
\hline 3 & 51 \\
\hline 4 & 52 \\
\hline 5 & 52 \\
\hline 6 & 53 \\
\hline 7 & 58 \\
\hline 8 & 60 \\
\hline 9 & 59 \\
\hline 10 & 62 \\
\hline 11 & 66 \\
\hline 12 & 67 \\
\hline 13 & 42 \\
\hline 14 & 41 \\
\hline 15 & 41 \\
\hline
\end{tabular}

\begin{tabular}{lc} 
DAY & POWER \\
\hline 16 & 41 \\
\hline 17 & 50 \\
\hline 18 & 66 \\
\hline 19 & 66 \\
\hline 20 & 66 \\
\hline 21 & 66 \\
\hline 22 & 66 \\
\hline 23 & 66 \\
\hline 24 & 67 \\
\hline 25 & 66 \\
\hline 26 & 66 \\
\hline 27 & 66 \\
\hline 28 & 66 \\
\hline 29 & 66 \\
\hline 30 & 66 \\
\hline 31 & 66 \\
\hline
\end{tabular}

26. Shutdowns Scheduled Over Next Six Months (Type, Date, Duration):

27. If Currently Shutdown, Estimated Startup Date:

Notes:

CUMULATIVE UNIT CAPACITY FACTOR (MDC NET) IS CALCULATED WITH A WEIGHTED AVERAGE. CUMULATIVE FORCED OUTAGE RATE IS CALCULATED

COMMENCING JANUARY 1, 1974. 


\begin{tabular}{|c|c|c|c|c|c|c|c|c|c|}
\hline No. & Date & Type & Hours & Reason & Method & LER Number & System & Component & Cause and Corrective Action To Prevent Recurrence \\
\hline $94-08$ & $12 / 13 / 94$ & $F$ & 0.0 & H & 5 & & & & $\begin{array}{l}\text { REDUCED LOAD DUE TO PACKING FAILURE ON \#2 REACTOR } \\
\text { FEEDWATER PUMP. }\end{array}$ \\
\hline
\end{tabular}
FEEDWATER PUMP.

\begin{tabular}{|c|c|}
\hline TYPE & REASON \\
\hline $\begin{array}{l}\text { F: Forced } \\
\text { S: Scheduled }\end{array}$ & $\begin{array}{l}\text { A-Equipment Fail lure } \\
\text { B-Maintenance or Test } \\
\text { C-Refuel ing } \\
\text { D-Regulatory Restriction } \\
\text { E-Operator Training \& } \\
\text { License Examination } \\
\text { F-Administrative } \\
\text { G-Operat ional Error } \\
\text { H-Other }\end{array}$ \\
\hline
\end{tabular}

\begin{tabular}{l} 
METHOD \\
\hline 1-Manual \\
2-Manual Scram \\
3-Auto Scram \\
4-Cont inued \\
5-Reduced Load \\
9-0ther
\end{tabular}

SYSTEM

IEEE Standard

805-1984 and/or

NUREG-0161 Exhibit $F$

5-Reduced Loed

9-0ther

\section{COMPONENT \\ IEEE Standard \\ 803A-1983 and/or
NUREG-0161 Exhibit H}




\section{FACILITY DESCRIPTION}

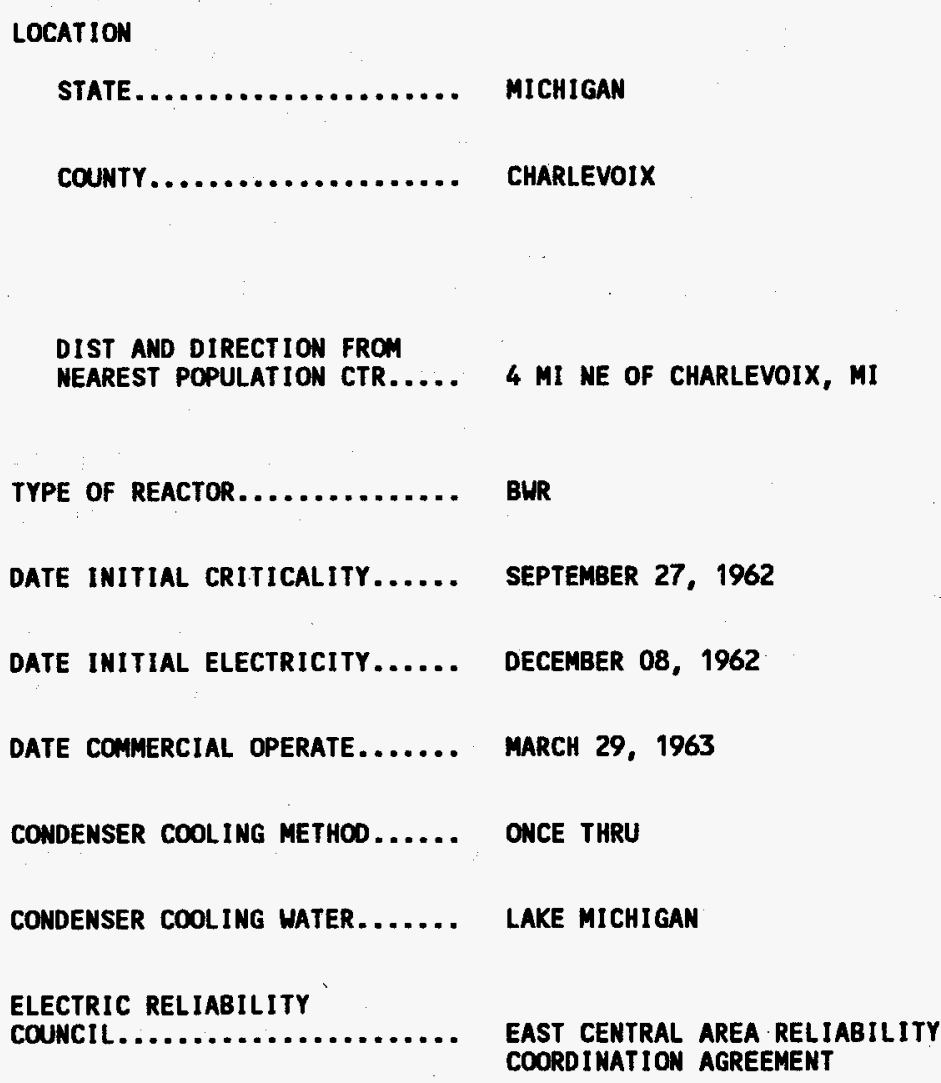

\section{UTILITY \& CONTRACTOR INFORMATION}

\section{UTILITY}

LICENSEE. CONSUMERS POUER CO.

CORPORATE ADDRESS

\section{WEST MICHIGAN AVENUE} JACKSON, MICHIGAN 49201

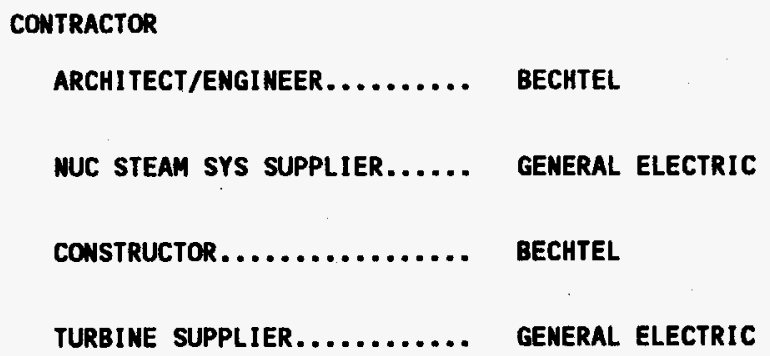

IE REGION RESPONSIBLE......... 3

IE RESIDENT IMSPECTOR......... ROY LEEMON

LICENSING PROJ MANAGER......... LEOHARD N. OLSHAN

DOCKET MUMBER.............. 50-155

LICENSE \& DATE ISSUANCE........ DPR O06, AUGUST 30, 1964 
1. Docket: $50-456$

OPERATING STATUS

2. Reporting Period: DECEMBER 1994

Outage + On-Line Hrs: $\quad 744.0$

3. Utility Contact: PAUL STANCZAK (815) 458-2801 EXT. 2486

4. Licensed Thermal Power (MWt):

5. Nameplate Rating (Gross MWe):

6. Design Electrical Rating (Net MWe):

7. Maximum Dependable Capacity (Gross MWe):

8. Maximum Dependable Capacity (Net MWe):

9. If Changes Occurred Above Since Last Report, Give Reasons:

10. Power Level To Which Restricted, If Any (Net MHe):

11. Reasons For Restrictions, If Any:

\begin{tabular}{|c|c|c|c|}
\hline & MONTH & YEAR & CUMULATIVE \\
\hline 12. Report Period Hrs & 744.0 & $8,760.0$ & $56,313.0$ \\
\hline 13. Hours Reactor Critical & 744.0 & $7,000.6$ & $44,598.8$ \\
\hline 14. Rx Reserve Shtdwn Hrs & 0.0 & 0.0 & $\overline{0.0}$ \\
\hline 15. Hrs Generator On-Line & 744.0 & $6,940.8$ & $43,966.3$ \\
\hline 16. Unit Reserve Shtdwn Hrs & 0.0 & 0.0 & $\overline{0.0}$ \\
\hline 17. Gross Therm Ener (MUH) & $2,312,753.5$ & $22,186,017.2$ & $137,439,297.7$ \\
\hline 18. Gross Elec Ener (MWH) & $809,844.0$ & $7,689,235.0$ & $46,503,822.0$ \\
\hline 19. Net Elec Ener (MUH) & $780,534.0$ & $7,390,003.0$ & $44,532,820.0$ \\
\hline 20. Unit Service Factor & 100.0 & 79.2 & 78.1 \\
\hline 21. Unit Avail Factor & 100.0 & 79.2 & 78.1 \\
\hline 22. Unit Cap Factor (MOC Net) & 93.7 & 75.3 & 70.6 \\
\hline 23. Unit Cap Factor (DER Net) & 93.7 & 75.3 & 70.6 \\
\hline 24. Unit Forced Outage Rate & 0.0 & 1.8 & 8.4 \\
\hline 25. Forced Outage Hours & 0.0 & 125.4 & $4,030.5$ \\
\hline
\end{tabular}

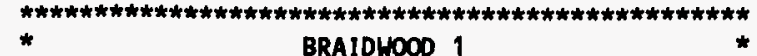
* BRA1DHOOO 1

AVERAGE DAILY POUER LEVEL (Net MWe)

\begin{tabular}{ll} 
DAY & PONER \\
\hline 1 & 1146 \\
\hline 3 & 1122 \\
\hline 4 & 328 \\
\hline 5 & 287 \\
\hline 6 & 810 \\
\hline 7 & 1143 \\
\hline 8 & 1144 \\
\hline 9 & 1145 \\
\hline 10 & 1149 \\
\hline 11 & 1034 \\
\hline 12 & 1034 \\
\hline 13 & 1148 \\
\hline 14 & 1149 \\
\hline 15 & 1147 \\
\hline
\end{tabular}

\begin{tabular}{ll} 
DAY & POWER \\
16 & 1140 \\
\hline 17 & 1145 \\
\hline 18 & 1145 \\
\hline 19 & 1144 \\
\hline 20 & 1141 \\
\hline 21 & 1146 \\
\hline 22 & 1145 \\
\hline 23 & 1145 \\
\hline 24 & 1076 \\
\hline 25 & 852 \\
\hline 26 & 1029 \\
\hline 27 & 1132 \\
\hline 28 & 1040 \\
\hline 29 & 1140 \\
\hline 30 & 1140 \\
\hline 31 & 1015 \\
\hline
\end{tabular}

26. Shutdowns Scheduled Over Next Six Months (Type, Date, Duration): FEBRUARY 19, 1995.

27. If Currently Shutdown, Estimated Startup Date:

Notes:

LICENSEE REVISED CUMULATIVE GROSS THERMAL ENERGY FROM $135,126,545.7$ TO $137,439,297.7$. 


\begin{tabular}{|c|c|c|c|c|c|c|c|c|c|}
\hline No. & Date & Type & Hours & Reason & Method & LER Number & System & Component & Cause and Corrective Action To Prevent Re \\
\hline 21 & $12 / 03 / 94$ & $\mathbf{s}$ & 0.0 & $\mathbf{H}$ & 5 & & FW & & FEEDWATER ISOLATION VALVE OIL CHANGE. \\
\hline 22 & $12 / 25 / 94$ & $\mathbf{s}$ & 0.0 & B & 5 & & & & $\begin{array}{l}\text { REDUCED LOAD AT THE REQUEST OF THE POWER } \\
\text { DISPATCHER TO FOLLOW SYSTEM REQUIREMENTS. }\end{array}$ \\
\hline
\end{tabular}

\begin{tabular}{ll} 
TYPE & REASON \\
\hline F: Forced & $\begin{array}{l}\text { A-Equipment Failure } \\
\text { B-Maintenance or Test } \\
\text { C-Refuel ing } \\
\text { D-Regulatory Restriction } \\
\text { E-Operator Training \& } \\
\text { License Examination } \\
\text { F-Administrative } \\
\text { G-Operational Error } \\
\text { H-Other }\end{array}$
\end{tabular}

METHOD
1-Manual
2-Manual Scram
3-Auto Scram
4-Cont inued
5-Reduced Load
9-0ther

SYSTEM

IEEE Standard

NUREG-0161 Exhibit F

\section{COMPONENT}

IEEE Standard

and/or

NUREG-0161 Exhibit H

G-Operational Error

H-Other 


\section{FACILITY DESCRIPTION}

LOCATION

STATE.................... ILLINOIS

COUNTY.................. WILL

\section{DIST AND DIRECTION FROM}

MEAREST POPULATION CTR..... 24 MI SSW OF JOLIET, IL

TYPE OF REACTOR.

PUR

DATE INITIAL CRITICALITY...... MAY 29, 1987

DATE INITIAL ELECTRICITY...... JULY 12, 1987

DATE COMMERCIaL OPERATE....... JULY 29, 1988

CONDENSER COOLING METHOD...... CC ART

CONDENSER COOLING WATER....... KANKAKEE RIVER

ELECTRIC RELIABILITY

COUNCIL.................. MID-AMERICA INTERPOOL NETWORK
UTILITY \& CONTRACTOR INFORMATION

UTILITY

LICENSEE................. COMMONHEALTH EDISON CO.

CORPORATE ADDRESS......... 1400 OPUS PL., OPUS MEST III

DOUITE 300 'S GROVE, ILLINOIS 60515

CONTRACTOR

ARCHITECT/ENGINEER.......... SARGENT \& LUMDY

NUC STEAM SYS SUPPLIER...... MESTINGHOUSE

CONSTRUCTOR.............. COMMONUEALTH EDISON

TURBINE SUPPLIER........... WESTINGHOUSE

REGULATORY INFORMATION

IE REGION RESPONSIBLE......... 3

IE RESIDENT INSPECTOR.......... STEVIE DU PONT

LICENSING PROJ MANAGER......... RAMIN R. ASSA

DOCKET NUMBER............. $50-456$

LICENSE \& DATE ISSUANCE....... NPF 072, JULY 02, 1987 
1. Docket: $50-457$

OPERATING STATUS

2. Reporting Period: DECEMBER 1994

Outage + On-Line Hrs: $\mathbf{7 4 4 . 0}$

3. Utility Contact: PAUL STANCZAK (815) 458-2801 EXT. 2486

4. Licensed Thermal Power (MWt):

5. Nameplate Rating (Gross Mue):

6. Design Electrical Rating (Net Mue):

7. Maximum Dependable Capacity (Gross MHe):

8. Maximum Dependable Capacity (Wet MHe):

9. If Changes Occurred Above since Last Report, Give Reasons:

10. Power Level To Which Restricted, If Any (Net MWe):

11. Reasons For Restrictions, If Any:

\begin{tabular}{|c|c|c|c|}
\hline & MONTH & YEAR & CUMULATIVE \\
\hline 12. Report Period Hrs & 744.0 & $8,760.0$ & $54,395.0$ \\
\hline 13. Hours Reactor Critical & 744.0 & $6,518.3$ & $44,832.5$ \\
\hline 14. Rx Reserve Shtdwn Hrs & 0.0 & 0.0 & 0.0 \\
\hline 15. Hrs Generator On-Line & 744.0 & $6,456.0$ & $44,442.3$ \\
\hline 16. Unit Reserve Shtdun Hrs & 0.0 & 0.0 & 0.0 \\
\hline 17. Gross Therm Ener (MUH) & $2,497,819.0$ & $20,040,146.7$ & $137,026,162.7$ \\
\hline 18. Gross Elec Ener (MWH) & $863,554.0$ & $6,905,966.0$ & $46,019,789.0$ \\
\hline 19. Net Elec Ener (MWH) & $833,881.0$ & $6,636,147.0$ & $44,102,730.0$ \\
\hline 20. Unit Service Factor & 100.0 & 73.7 & 81.7 \\
\hline 21. Unit Avail Factor & 100.0 & 73.7 & 81.7 \\
\hline 22. Unit Cap Factor (MDC Net) & 100.1 & 67.6 & 72.4 \\
\hline 23. Unit Cap Factor (DER Net) & 100.1 & 67.6 & 72.4 \\
\hline 24. Unit Forced Outage Rate & 0.0 & 17.0 & 5.9 \\
\hline 25. Forced Outage Hours & 0.0 & 1325.3 & $2,771.3$ \\
\hline
\end{tabular}

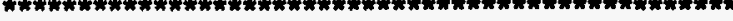

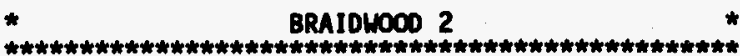

AVERAGE DAILY PONER LEVEL (Net MMe)

\begin{tabular}{ll} 
DAY & PONER \\
\hline 1 & 1118 \\
\hline 2 & 1125 \\
\hline 3 & 1129 \\
\hline 4 & 1128 \\
\hline 5 & 1132 \\
\hline 6 & 1136 \\
\hline 7 & 1136 \\
\hline 8 & 1137 \\
\hline 9 & 1135 \\
\hline 10 & 1134 \\
\hline 11 & 1134 \\
\hline 12 & 1135 \\
\hline 13 & 1133 \\
\hline 14 & 1134 \\
\hline 15 & 1132 \\
\hline
\end{tabular}

\begin{tabular}{ll} 
DAY & POWER \\
\hline 16 & 1133 \\
\hline 17 & 1133 \\
\hline 18 & 1044 \\
\hline 19 & 1131 \\
\hline 20 & 1129 \\
\hline 21 & 1133 \\
\hline 22 & 1132 \\
\hline 23 & 1131 \\
\hline 24 & 1130 \\
\hline 25 & 1109 \\
\hline 26 & 1124 \\
\hline 27 & 1131 \\
\hline 28 & 1131 \\
\hline 29 & 1129 \\
\hline 30 & 969 \\
\hline 31 & 1064 \\
\hline
\end{tabular}

26. Shutdowns Scheduled Over Mext Six Months (Type, Date, Duration):

27. If Currently Shutdown, Estimated Startup Date:

Notes:

LICENSEE REVISED CUMULATIVE GROSS THERMAL EMERGY FROM $134,528,343.8$ TO $137,026,162.7$. 


\begin{tabular}{l} 
REASON \\
\hline A-Equipment Failure \\
B-Maintenance or Test \\
C-Refuel ing \\
D-Regulatory Restriction \\
E-Operator Training \& \\
License Examination \\
F-Administrat ive \\
G-Operational Error \\
H-Other
\end{tabular}

\section{METHOD}

1-Manual

2-Manual Scram

3-Auto Scram

4-Cont inued

5-Reduced Load

9-0ther

\section{SYSTEM}

IEEE Standard

805-1984 and/or

NUREG-0161 Exhibit F
COMPONENT

IEEE Standard

803A-1983 and/or

NUREG-0161 Exhibit

G-Operational Error

H-other 
FACILITY DESCRIPTION

LOCATION

STATE.................. ILLINOIS
COUNTY................ WILL

DIST AND DIRECTION FROM

NEAREST POPULATION CTR..... 24 MI SSW OF JOLIET, IL

TYPE OF REACTOR

DATE INITIAL CRITICALITY.

DATE INITIAL ELECTRICITY...... MAY 25, 1988

DATE COMMERCIAL OPERATE....... OCtOber 17, 1988

CONDENSER COOLING METHOD...... CCART

CONDENSER COOLING MATER....... KAMKAKEE RIVER

ELECTRIC RELIABILITY

COUNCIL........... MID-AMERICA INTERPOOL METWORK

\section{UTILITY \& CONTRACTOR INFORMATION}

UTILITY

LICENSEE.

CORPORATE ADDRESS.

COMMONUEALTH EDISON CO.

1400 OPUS PL., OPUS WEST III

SUITE 300
DOWNER'S GROVE, ILLINOIS 60515

\section{CONTRACTOR}

ARCHITECT/ENGIMEER.

SARGENT \& LUNDY

NUC STEAM SYS SUPPLIER...... MESTIMGHOUSE

CONSTRUCTOR................ COMMONHEALTH EDISON

TURBINE SUPPLIER........... MESTINGHOUSE

\section{REGULATORY INFORMATION}

IE REGION RESPONSIBLE........ 3

IE RESIDENT INSPECTOR.......... STEVIE DU PONT

LICENSING PROJ MANAGER......... RAMIN R. ASSA

DOCKET NUMBER

$50-457$

LICENSE \& DATE ISSUANCE

NPF 077, MAY 20, 1988 


\section{Docket: $50-259$}

OPERATING STATUS

2. Reporting Period: DECEMBER 1994

Outage + On-Line Hrs:

0.0

3. Utility Contact: T. R. SMITH (205) 729-2955

4. Licensed Thermal Power (Mut):

5. Nameplate Rating (Gross Mue):

6. Design Electrical Rating (Net MHe):

7. Maximum Dependable Capacity (Gross MHe):

8. Maximum Dependable Capacity (Net MWe):

$\frac{3293}{\frac{1152}{1065}}$

9. If Changes Occurred Above Since Last Report, Give Reasons:

10. Power Level To thich Restricted, If Any (Net Mue):

11. Reasons For Restrictions, If Any:

\begin{tabular}{|c|c|c|c|}
\hline & MONTH & YEAR & CUMULATIVE \\
\hline 12. Report Period Hrs & 0.0 & 0.0 & $95,743.0$ \\
\hline 13. Hours Reactor Critical & 0.0 & 0.0 & $59,521.0$ \\
\hline 14. Rx Reserve Shtdwn Hrs & 0.0 & 0.0 & $6,997.0$ \\
\hline 15. Hrs Generator On-Line & 0.0 & 0.0 & $58,267.0$ \\
\hline 16. Unit Reserve Shtdwn Hrs & 0.0 & 0.0 & 0.0 \\
\hline 17. Gross Therm Ener (MNH) & 0.0 & 0.0 & $168,066,787.0$ \\
\hline 18. Gross Elec Ener (MUH) & 0.0 & 0.0 & $55,398,130.0$ \\
\hline 19. Net Elec Ener (MUH) & 0.0 & 0.0 & $53,796,427.0$ \\
\hline 20. Unit Service Factor & 0.0 & 0.0 & 60.9 \\
\hline 21. Unit Avail Factor & 0.0 & 0.0 & 60.9 \\
\hline 22. Unit Cap Factor (MDC Net) & 0.0 & 0.0 & 52.8 \\
\hline 23. Unit Cap Factor (DER Net) & 0.0 & 0.0 & 52.8 \\
\hline 24. Unit Forced Outage Rate & 0.0 & 0.0 & 25.6 \\
\hline 25. Forced Outage Hours & 0.0 & 0.0 & $20,022.0$ \\
\hline
\end{tabular}

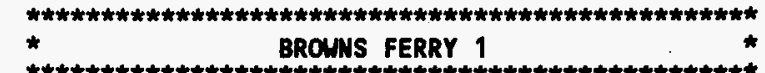

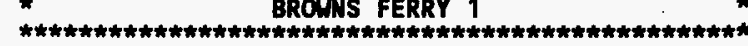

AVERAGE DAILY PONER LEVEL (Net MUe)

\begin{tabular}{lc} 
DAY & POUER \\
\hline 1 & 0 \\
\hline 2 & 0 \\
\hline 3 & 0 \\
\hline 4 & 0 \\
\hline 5 & 0 \\
\hline 6 & 0 \\
\hline 7 & 0 \\
\hline 8 & 0 \\
\hline 9 & 0 \\
\hline 10 & 0 \\
\hline 11 & 0 \\
\hline 12 & 0 \\
\hline 13 & 0 \\
\hline 14 & 0 \\
\hline 15 & 0 \\
\hline
\end{tabular}

\begin{tabular}{ll} 
DAY & PONER \\
\hline 16 & 0 \\
\hline 17 & 0 \\
\hline 18 & 0 \\
\hline 19 & 0 \\
\hline 20 & 0 \\
\hline 21 & 0 \\
\hline 22 & 0 \\
\hline 23 & 0 \\
\hline 24 & 0 \\
\hline 25 & 0 \\
\hline 26 & 0 \\
\hline 27 & 0 \\
\hline 28 & 0 \\
\hline 29 & 0 \\
\hline 30 & 0 \\
\hline 31 & \\
\hline
\end{tabular}

26. Shutdowns Scheduled Over Next Six Months (Type, Date, Duration):

27. If Currently Shutdown, Estimated Startup Date:

Notes:

LICENSEE HAS SUSPENDED THE ACCRUAL OF REPORTING DATA TO REFLECT THE ASSIGNMENT OF ADMINISTRATIVE HOLD EFFECTIVE JUNE 1, 1985. 


$\begin{array}{llllll}1 & 06 / 01 / 85 & S & 744.0 & F & 4\end{array}$

\begin{tabular}{ll} 
TYPE & REASON \\
\hline F: Forced & $\begin{array}{l}\text { A-Equipment Failure } \\
\text { B-Maintenance or Test } \\
\text { C-Refuel ing } \\
\text { D-Regulatory Restriction } \\
\text { E-Operator Training \& } \\
\text { License Examination } \\
\text { F-Administrative } \\
\text { G-Operational Error } \\
\text { H-other }\end{array}$
\end{tabular}

\begin{tabular}{l} 
METHOD \\
\hline 1-Manual \\
2-Manual Scram \\
3-Auto Scram \\
4-Cont inued \\
5-Reduced Load \\
9-Other
\end{tabular}

\section{SYSTEM}

IEEE Standard

805-1984 and/or

NUREG-0161 Exhibit F
COMPONENT

IEEE Standard

803A-1983 and/o

NUREG-0161 Exhibit H 


\section{FACILITY DESCRIPTION}

LOCATION

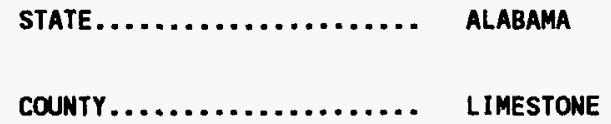

\section{UTILITY \& CONTRACTOR INFORMATION}

UTILITY

LICENSEE.

TENNESSEE VALLEY AUTHORITY

CORPORATE ADDRESS.

400 WEST SUMMIT HILL DRIVE KNOXVILLE, TENNESSEE 37933

\section{CONTRACTOR}

ARCHITECT/ENGINEER......... TENMESSEE VALLEY AUTHORITY

NUC STEAM SYS SUPPLIER...... GENERAL ELECTRIC

CONSTRUCTOR............... TEMMESSEE VALLEY AUTHORITY

TURBINE SUPPLIER.......... GENERAL ELECTRIC

\section{REGULATORY INFORMATION}

IE REGION RESPONSIBLE......... 2

IE RESIDENT INSPECTOR......... LEONARD MERT

LICENSING PROJ MANAGER........ DAVID C. TRIMBLE JR.

DOCKET NUMBER

50-259

LICENSE \& DATE ISSUANCE.
DPR 033, DECEMBER 20, 1973 
1. Docket: $50-260$

OPERATING STATUS

2. Reporting Period: DECEMBER 1994

Outage + On-Line Hrs:

744.0

3. Utility Contact: T. R. SMITH (205) 729-2955

4. Licensed Thermal Power (MWt):

5. Nameplate Rating (Gross MWe):

6. Design Electrical Rating (Net MWe):

7. Maximum Dependable Capacity (Gross MHe):

8. Maximum Dependable Capacity (Net MWe):

\begin{tabular}{c}
3293 \\
\hline 1152 \\
\hline 1065 \\
\hline 1098 \\
\hline 1065
\end{tabular}

9. If Changes Occurred Above since Last Report, Give Reasons:

10. Power Level To Which Restricted, If Any (Net MWe):

11. Reasons For Restrictions, If Any:

\begin{tabular}{|c|c|c|c|}
\hline & MONTH & YEAR & CUNULAT IVE \\
\hline 12. Report Period Hrs & 744.0 & $8,760.0$ & $122,071.0$ \\
\hline 13. Hours Reactor Critical & 729.0 & $7,310.0$ & $82,166.2$ \\
\hline 14. Rx Reserve Shtdwn Hrs & 0.0 & 0.0 & $14,200.0$ \\
\hline 15. Hrs Generator On-Line & 711.0 & $7,234.0$ & $79,859.2$ \\
\hline 16. Unit Reserve Shtdun Hrs & 0.0 & 0.0 & 0.0 \\
\hline 17. Gross Therm Ener (MWH) & $2,148,854.0$ & $22,621,315.0$ & $231,168,916.0$ \\
\hline 18. Gross Elec Ener (MWH) & $726,260.0$ & $7,535,263.0$ & $76,743,181.0$ \\
\hline 19. Net Elec Ener (MUH) & $708,446.0$ & $7,345,174.0$ & $74,589,836.0$ \\
\hline 20. Unit Service Factor & 95.6 & 82.6 & 65.4 \\
\hline 21. Unit Avail Factor & 95.6 & 82.6 & 65.4 \\
\hline 22. Unit Cap Factor (MDC Net) & 89.4 & 78.7 & 57.4 \\
\hline 23. Unit Cap factor (DER Net) & 89.4 & 78.7 & 57.4 \\
\hline 24. Unit Forced Outage Rate & 2.7 & 2.2 & 17.5 \\
\hline 25. Forced Outage Hours & 20.0 & 166.0 & $16,956.5$ \\
\hline
\end{tabular}

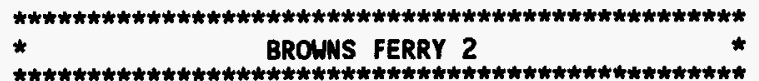

AVERAGE DAILY POWER LEVEL (Net MWe)

\begin{tabular}{lc} 
DAY & POWER \\
\hline 1 & 125 \\
\hline 2 & 154 \\
\hline 3 & 367 \\
\hline 4 & 519 \\
\hline 5 & 778 \\
\hline 6 & 745 \\
\hline 7 & 915 \\
\hline 8 & 1048 \\
\hline 9 & 955 \\
\hline 10 & 1067 \\
\hline 11 & 1090 \\
\hline 12 & 1095 \\
\hline 13 & 1095 \\
\hline 14 & 1096 \\
\hline 15 & 1094 \\
\hline
\end{tabular}

\begin{tabular}{ll} 
DAY & PONER \\
\hline 16 & 1095 \\
\hline 17 & 1098 \\
\hline 18 & 1091 \\
\hline 19 & 1091 \\
\hline 20 & 1029 \\
\hline 21 & 1054 \\
\hline 22 & 1079 \\
\hline 23 & 1092 \\
\hline 24 & 1093 \\
\hline 25 & 1092 \\
\hline 26 & 1091 \\
\hline 27 & 1098 \\
\hline 28 & 1098 \\
\hline 29 & 1097 \\
\hline 30 & 1091 \\
\hline 31 & 1087 \\
\hline
\end{tabular}

26. Shutdowns Scheduled Over Next Six Months (Type, Date, Duration):

27. If Currently Shutdown, Estimated Startup Date:

Notes:

REVISED CUMULATIVE NET ELECTRICAL ENERGY TO REFLECT THE UNIT 2 ADMINISTRATIVE HOLD STATUS FROM JUNE 1, 1985 UNTIL THE RESTART

FOR CYCLE 6 ON MAY 24, 1991. 
UNIT SHUTDOWNS

A N 0

POWE

REDUCTIONS

-

BROWNS FERRY 2 ,

\begin{tabular}{|c|c|c|c|c|c|c|c|c|c|c|}
\hline No. & Date & Type & Hours & Reason & Method & LER & Number & System & Component & Cause and Corrective Action To Prevent Recurrence \\
\hline
\end{tabular}

$5 \quad 11 / 28 / 94$

$6 \quad 12 / 02 / 94$

13.0

4

F 20.0

A 3

JJ

TIS

PLANNED POST OUTAGE ACTIVITIES.

AUTOMATIC SCRAM CAUSED BY BALANCE OF PLANT EQUIPMENT FAILURE.

\begin{tabular}{l} 
TYPE \\
\hline F: Forced \\
S: Scheduled
\end{tabular}

\section{REASON}

A-Equipment Failure

B-Maintenance or Test

C-Refuel ing

D-Regulatory Restriction

-Operator Training \&

License Examinat

G-Operational Error

G-Operatio
H-Other
METHOO

1-Manual

2-Manual Scram

3-Auto Scram

4-Continued

5-Reduced Load

9-other

\section{SYSTEM}

IEEE Standard

NUREG-0161 Exhibit F

\section{COMPONENT}

IEEE Standard

NUREG-0161 Exhibit H 


\section{FACILITY DESCRIPTION}

LOCATION

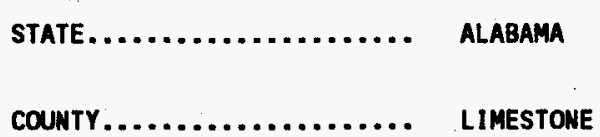

\section{DIST AND DIRECTION FROM} MEAREST POPULATION CTR.

TYPE OF REACTOR

10 MI NW OF DECATUR, AL

DATE INITIAL CRITICALITY...... JULY 20, 1974

DATE IMITIAL ELECTRICITY...... AUGUST 28, 1974

DATE COMMERCIAL OPERATE....... MARCH 01, 1975

CONDENSER COOLING METHOD...... ONCE THRU

CONDENSER COOLING WATER....... TENNESSEE RIVER

ELECTRIC RELIABILITY

COUNCIL................... SOUTHEASTERN ELECTRIC RELIABILITY COUNCIL

\section{UTILITY \& CONTRACTOR INFORMATION}

\section{UTILITY}

LICENSEE

CORPORATE ADDRESS

TENNESSEE VALLEY AUTHORITY

400 UEST SUMMIT HILL DRIVE KNOXVILLE, TENNESSEE 37933

\section{CONTRACTOR}

ARCHITECT/ENGIMEER.

NUC STEAM SYS SUPPLIER.

CONSTRUCTOR.

TURBINE SUPPLIER

\section{REGULATORY INFORMATION}

IE REGION RESPONSIBLE..........

IE RESIDENT INSPECTOR......... LEONARD MERT

LICENSING PROJ MANAGER......... DAVID C. TRIMBLE JR.

DOCKET NUMBER............. $50-260$

LICENSE \& DATE ISSUANCE........ DPR 052, AUGUST 02, 1974 
1. Docket: $50-296$

O PERATING

STATUS

2. Reporting Period: DECEMBER 1994

Outage + On-Line Hrs:

0.0

3. Utility contact: T. R. SMITH (205) 729-2955

4. Licensed Thermal Power (MWt):

5. Nameplate Rating (Gross MWe):

6. Design Electrical Rating (Net MWe):

7. Maximum Dependable Capacity (Gross MHe):

8. Maximum Dependable Capacity (Net MUe):

\begin{tabular}{c}
$\frac{3293}{1152}$ \\
\hline 1065 \\
\hline 0 \\
\hline 0 \\
\hline
\end{tabular}

9. If Changes Occurred Above since Last Report, Give Reasons:

10. Power Level To Which Restricted, If Any (Net MWe):

11. Reasons for Restrictions, If Any:

MONTH

12. Report Period Hrs

13. Hours Reactor Critical

14. Rx Reserve Shtdwn Hrs

15. Hrs Generator On-Line

16. Unit Reserve Shtdwn Hrs

17. Gross Therm Ener (MWH)

18. Gross Elec Ener (MUH)

19. Net Elec Ener (MWH)

20. Unit Service Factor

21. Unit Avail Factor

22. Unit Cap factor (MDC Net)

23. Unit Cap Factor (DER Net)

24. Unit Forced Outage Rate

25. Forced Outage Hours

$$
\text { }
$$

YEAR

CUMULAT IVE

\begin{tabular}{|c|c|c|}
\hline 0.0 & 0.0 & $73,055.0$ \\
\hline 0.0 & 0.0 & $45,306.0$ \\
\hline 0.0 & 0.0 & $5,150.0$ \\
\hline 0.0 & 0.0 & $44,195.0$ \\
\hline 0.0 & 0.0 & 0.0 \\
\hline 0.0 & 0.0 & $131,868,267.0$ \\
\hline 0.0 & 0.0 & $43,473,760.0$ \\
\hline 0.0 & 0.0 & $42,114,009.0$ \\
\hline 0.0 & 0.0 & 60.5 \\
\hline 0.0 & 0.0 & 60.5 \\
\hline 0.0 & 0.0 & 54.2 \\
\hline 0.0 & 0.0 & 54.2 \\
\hline 0.0 & 0.0 & 21.6 \\
\hline 0.0 & 0.0 & $12,155.0$ \\
\hline
\end{tabular}

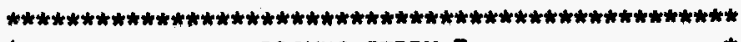

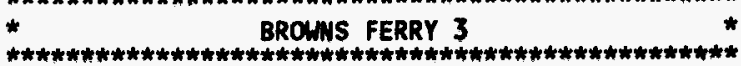

AVERAGE DAILY PONER LEVEL (Net MHe)

\begin{tabular}{lc} 
DAY & PQNER \\
\hline 1 & 0 \\
\hline 2 & 0 \\
\hline 3 & 0 \\
\hline 4 & 0 \\
\hline 5 & 0 \\
\hline 6 & 0 \\
\hline 7 & 0 \\
\hline 8 & 0 \\
\hline 9 & 0 \\
\hline 10 & 0 \\
\hline 11 & 0 \\
\hline 12 & 0 \\
\hline 13 & 0 \\
\hline 14 & 0 \\
\hline 15 & 0 \\
\hline
\end{tabular}

\begin{tabular}{cc} 
DAY & PONER \\
\hline 16 & 0 \\
\hline 17 & 0 \\
\hline 18 & 0 \\
\hline 19 & 0 \\
\hline 20 & 0 \\
\hline 21 & 0 \\
\hline 22 & 0 \\
\hline 23 & 0 \\
\hline 24 & 0 \\
\hline 25 & 0 \\
\hline 26 & 0 \\
\hline 27 & 0 \\
\hline 28 & 0 \\
\hline 29 & 0 \\
\hline 30 & 0 \\
\hline 31 & 0 \\
\hline
\end{tabular}

26. Shutdowns Scheduled Over Next Six Months (Type, Date, Duration):

27. If Currently Shutdown, Estimated Startup Date:

Notes:

LICENSEE HAS SUSPENDED THE ACCRUAL OF REPORTING DATA TO REFLECT THE ASSIGNMENT OF ADMINISTRATIVE HOLD EFFECTIVE JUNE 1 , 1985. 


\begin{tabular}{|c|c|c|c|c|c|c|c|c|c|}
\hline No. & Date & Type & Hours & Reason & Method & LER Number. & System & Component & Cause and Corrective Action To Prevent Recurrence \\
\hline 1 & $06 / 01 / 85$ & $\mathbf{s}$ & 744.0 & $\mathbf{F}$ & 4 & . & & & ADMINISTRATIVE HOLD TO RESOLVE VARIOUS TVA AND \\
\hline
\end{tabular}

NRC CONCERNS.

TYPE

F: Forced

s: Scheduled

\section{REASON}

A-Equipment Failure

B-Maintenance or Test

C-Refuel ing

D-Regulatory Restriction

E-Operator Training \&

License Examination

F-Administrative

G-Operational Error

$\mathrm{H}$-other

\begin{tabular}{l} 
METHOD \\
\hline 1-Manual \\
2-Manual Scram \\
3-Auto Scram \\
4-Cont inued \\
5-Reduced Load \\
9-Other
\end{tabular}

SYSTEM

IEEE Standard

805-1984 and/or

NUREG-0161 Exhibit F

COMPONENT

IEEE Standard

803A-1983 and/or

NUREG-0161 Exhibit H

5-Reduced Lad 


\section{FACILITY DESCRIPTION}

\section{LOCATION}

STATE.

countr.

\section{DIST AND DIRECTION FROM}

NEAREST POPULATION CTR .....

TYPE OF REACTOR.

BWR

DATE INITIAL CRITICALITY....... AUGUST 08, 1976

DATE initial ELECTRICITY...... SEPTENBER 12, i976

DATE COMMERCIAL OPERATE....... MARCH 01, 1977

CONDENSER COOLING METHOD...... ONCE THRU

CONOENSER COOLING WATER....... TENNESSEE RIVER

ELECTRIC RELIABILITY

COUNCIL.................. SOUTHEASTERN ELECTRIC
UTILITY \& CONTRACTOR INFORMATION

\section{UTILITY}

LICENSEE................. TENMESSEE VALLEY AUTHORITY

CORPORATE ADDRESS

\section{WEST SUMMIT HILL DRIVE} KNOXVILLE, TENMESSEE 37933

\section{CONTRACTOR}

ARCHITECT/ENGINEER......... TENMESSEE VALLEY AUTHORITY

NUC STEAM SYS SUPPLIER...... GEMERAL ELECTRIC

CONSTRUCTOR............... TENMESSEE VALLEY AUTHORITY

TURBINE SUPPLIER........... GENERAL ELECTRIC

\section{REGULATORY INFORMATION}

IE REGION RESPONSIBLE........ 2

IE RESIDENT INSPECTOR

LEONARD WERT

LICENSING PROJ MANAGER.

JOSEPH F. WILLIAMS

DOCKET NUMBER.

$50-296$

LICENSE \& DATE ISSUANCE.

DPR 068, AUGUST 18, 1976 
1. Docket: $50-325$

OPERATING STATUS

2. Reporting Period: DECEMBER 1994

Outage + On-Line Hrs: $\quad 744.0$

3. Utility Contact: FRANCES HARRISON (910) 457-2756

4. Licensed Thermal Power (MUt):

5. Nameplate Rating (Gross MHe):

6. Design Electrical Rating (Net MWe):

7. Maximum Dependable Capacity (Gross MHe):

8. Maximum Dependable Capacity (Net MWe):

9. If Changes Occurred Above Since Last Report, Give Reasons:

10. Power Level To Which Restricted, If Any (Net MWe):

11. Reasons For Restrictions, If Any:

\begin{tabular}{|c|c|c|c|}
\hline & MONTH & & CUMULATIVE \\
\hline 12. Report Period Hrs & 744.0 & $8,760.0$ & $155,952.0$ \\
\hline 13. Hours Reactor Critical & 744.0 & $7,990.0$ & $95,871.3$ \\
\hline 14. Rx Reserve Shtdwn Hrs & 0.0 & 0.0 & $1,647.1$ \\
\hline 15. Hrs Generator On-Line & 744.0 & $7,755.7$ & $91,957.0$ \\
\hline 16. Unit Reserve Shtchun Hrs & 0.0 & 0.0 & 0.0 \\
\hline 17. Gross Therm Ener (MUH) & $1,763,525.6$ & $18,513,783.1$ & $201,535,726.6$ \\
\hline 18. Gross Elec Ener (MWH) & $587,155.0$ & $6,140,440.0$ & $66,159,725.0$ \\
\hline 19. Net Elec Ener (МนH) & $570,872.0$ & $5,950,090.0$ & $63,599,039.0$ \\
\hline 20. Unit Service Factor & 100.0 & 88.5 & 59.0 \\
\hline 21. Unit Avail Factor & 100.0 & 88.5 & 59.0 \\
\hline 22. Unit Cap factor (MDC Net) & 100.0 & 88.6 & 52.0 \\
\hline 23. Unit Cap Factor (DER Net) & 93.5 & 82.7 & 49.7 \\
\hline 24. Unit Forced Outage Rate & 0.0 & 0.2 & 14.9 \\
\hline 25. Forced Outage Hours & 0.0 & 18.6 & $16,106.4$ \\
\hline
\end{tabular}

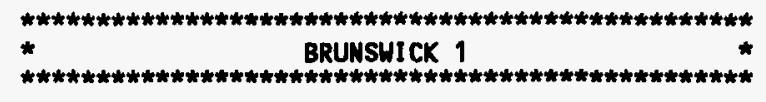

AVERAGE DAILY POUER LEVEL (Net MUe)

\begin{tabular}{|c|c|c|c|}
\hline DAY & POUER & DAY & POUER \\
\hline 1 & 791 & 16 & 790 \\
\hline 2 & 791 & 17 & 660 \\
\hline 3 & 790 & 18 & 729 \\
\hline 4 & 773 & 19 & 791 \\
\hline 5 & 789 & 20 & 791 \\
\hline 6 & 789 & 21 & 515 \\
\hline 7 & 788 & 22 & 789 \\
\hline 8 & 789 & 23 & 790 \\
\hline 9 & 790 & 24 & 791 \\
\hline 10 & 789 & 25 & 792 \\
\hline 11 & 789 & 26 & 791 \\
\hline 12 & 709 & 27 & 791 \\
\hline 13 & 650 & 28 & 790 \\
\hline 14 & 789 & 29 & 791 \\
\hline 15 & 790 & 30 & 791 \\
\hline & & 31 & 790 \\
\hline
\end{tabular}

26. Shutdowns Scheduled Over Mext Six Months (Type, Date, Duration):

27. If Currently Shutdown, Estimated Startup Date:

Notes:

CUMULATIVE UNIT CAPACITY FACTOR (MDC NET) IS CALCULATED WITH A WEIGHTED AVERAGE. 


W M S A N D P OWE R RED U C T I O M S

\begin{tabular}{|c|c|c|c|c|c|c|c|c|c|}
\hline No. & Date & Type & Hours & Reason & Method & LER Number & System & Component & Cause and Corrective Action To Prevent Recurrence \\
\hline $94-071$ & $12 / 12 / 94$ & $\mathbf{F}$ & 0.0 & A & 5 & & EG & GENERG & $\begin{array}{l}\text { IA UNINTERRUPTIBLE PONER SUPPLY TRIPPED. } \\
\text { REPLACED THE VOLTAGE SENSOR ASSEMBLY IN THE IA } \\
\text { UPS. }\end{array}$ \\
\hline $94-072$ & $12 / 17 / 94$ & $\mathbf{F}$ & 0.0 & A & 5 & & SD & COND & $\begin{array}{l}\text { REDUCED REACTOR PONER TO REPAIR LEAK ON B-S } \\
\text { WATERBOX. }\end{array}$ \\
\hline $94-073$ & $12 / 20 / 94$ & $\mathbf{F}$ & 0.0 & A & 5 & & SD & COND & $\begin{array}{l}\text { REDUCED REACTOR POWER TO REPAIR TUBE LEAKS ON B-S } \\
\text { HATERBOX. }\end{array}$ \\
\hline
\end{tabular}

\begin{tabular}{|c|c|}
\hline TYPE & REASOW \\
\hline $\begin{array}{l}\text { F: Forced } \\
\text { S: Scheduled }\end{array}$ & $\begin{array}{l}\text { A-Equipment Failure } \\
\text { B-Maintenance or Test } \\
\text { C-Refueling } \\
\text { D-Regulatory Restriction } \\
\text { E-Operator Training \& } \\
\text { License Examination } \\
\text { F-Administrative } \\
\text { G-Operational Error } \\
\text { H-Other }\end{array}$ \\
\hline
\end{tabular}

\begin{tabular}{l} 
METHOD \\
\hline 1-Manual \\
2-Manual Scram \\
3-Auto Scram \\
4-Cont inued \\
5-Reduced Load \\
9-0ther
\end{tabular}

\section{SYSTEM}

IEEE Standard

$805-1984$ and/or
NUREG-0161 Exhibit F
COMPONENT

IEEE Standard

NUREG-0161 Exhibit H

G-Operational Error

5-Reduced Load 


\section{FACILITY DESCRIPTION}

LOCATION

STATE.$\ldots \ldots \ldots \ldots \ldots \ldots \ldots \ldots$
COUNTY................... MORTH CAROLIMA

DIST AND DIRECTION FROM

MEAREST POPULATION CTR..... 2 MI N OF SOUTHPORT, NC

TYPE OF REACTOR

BUR

DATE INITIAL CRITICALITY...... OCTOBER 08, 1976

DATE INITIAL ELECTRICITY...... DECEMBER 04, 1976

date commercial operate....... MarCh 18, 1977

CONDENSER COOLING METHOD...... ONCE THRU

CONDENSER COOLING WATER....... CAPE FEAR RIVER

ELECTRIC RELIABILITY

COUNCIL.................. SOUTHEASTERN ELECTRIC RELIABILITY COUNCIL

\section{UTILITY \& CONTRACTOR INFORMATION}

UTILITY

LICENSEE.

CAROLIMA POWER \& LIGHT CO.

CORPORATE ADDRESS.

P.O. BOX 1551

P.O. BOX 1551
RALEIGH, NORTH CAROLIMA 27602

\section{CONTRACTOR}

ARCHITECT/ENGINEER.

UNITED ENG. \& CONSTRUCTORS

HUC STEAM SYS SUPPLIER.......

GENERAL ELECTRIC

CONSTRUCTOR.

BRON \& ROOT

TURBINE SUPPLIER

General ELECTRIC

\section{REGULATORY IMFORMATION}

IE REGION RESPONSIBLE.........

IE RESIDENT INSPECTOR

CHARLES PATTERSON

LICENSING PROJ MANAGER

PATRICK D. MILANO

DOCKET NUMBER

50-325

LICENSE \& DATE ISSUANCE. 
1. Docket: $50-324$

2. Reporting Period: DECEMBER 1994

OPERATING STATUS

3. Utility Contact:

Outage + On-Line Hrs:

744.0

4. Licensed Thermal Power (MWt):

5. Nameplate Rating (Gross MWe):

6. Design Electrical Rating (Net MWe):

7. Maximum Dependable Capacity (Gross MWe):

8. Maximum Dependable Capacity (Net MHe):

\begin{tabular}{c}
\hline 2436 \\
\hline 867 \\
\hline 821 \\
\hline 782 \\
\hline 754
\end{tabular}

9. If Changes Occurred Above Since Last Report, Give Reasons:

10. Power Level To Which Restricted, If Any (Net MWe):

11. Reasons For Restrictions, If Any:

\begin{tabular}{|c|c|c|c|}
\hline & MONTH & YEAR & CUMULATIVE \\
\hline 12. Report Period Hrs & 744.0 & $8,760.0$ & $167,976.0$ \\
\hline 13. Hours Reactor Critical & 744.0 & $6,549.0$ & $104,555.6$ \\
\hline 14. Rx Reserve Shtuinn Hirs & 0.0 & 0.0 & 0.0 \\
\hline 15. Hrs Generator On-Line & 744.0 & $6,437.2$ & $99,117.6$ \\
\hline 16. Unit Reserve Shtdwn Hrs & 0.0 & 0.0 & 0.0 \\
\hline 17. Gross Therm Ener (MWH) & $1,802,772.5$ & $15,353,546.2$ & $212,250,334.7$ \\
\hline 18. Gross Elec Ener (MUH) & $587,080.0$ & $4,970,307.0$ & $68,649,621.0$ \\
\hline 19. Net Elec Ener (MUH) & $570,646.0$ & $4,809,206.0$ & $65,833,259.0$ \\
\hline 20. Unit Service Factor & 100.0 & 73.5 & 59.0 \\
\hline 21. Unit Avail Factor & 100.0 & 73.5 & 59.0 \\
\hline 22. Unit Cap Factor (MDC Net) & 101.7 & 72.8 & 50.1 \\
\hline 23. Unit Cap Factor (DER Net) & 93.4 & 66.9 & 47.7 \\
\hline 24. Unit Forced Outage Rate & 0.0 & 0.0 & 12.0 \\
\hline 25. Forced Outage Hours & 0.0 & 0.0 & $13,562.5$ \\
\hline
\end{tabular}

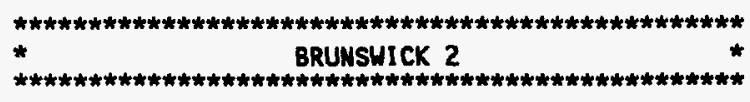

AVERAGE DAILY POWER LEVEL (Net MWe)

\begin{tabular}{ll} 
DAY & PONER \\
\hline 1 & 770 \\
\hline 2 & 770 \\
\hline 3 & 769 \\
\hline 4 & 768 \\
\hline 5 & 769 \\
\hline 6 & 769 \\
\hline 7 & 769 \\
\hline 8 & 769 \\
\hline 9 & 770 \\
\hline 10 & 770 \\
\hline 11 & 770 \\
\hline 12 & 771 \\
\hline 13 & 772 \\
\hline 14 & 771 \\
\hline 15 & 771 \\
\hline
\end{tabular}

\begin{tabular}{ll} 
DAY & POUER \\
\hline 16 & 771 \\
\hline 17 & 770 \\
\hline 18 & 771 \\
\hline 19 & 771 \\
\hline 20 & 771 \\
\hline 21 & 771 \\
\hline 22 & 771 \\
\hline 23 & 742 \\
\hline 24 & 683 \\
\hline 25 & 772 \\
\hline 26 & 773 \\
\hline 27 & 773 \\
\hline 28 & 772 \\
\hline 29 & 773 \\
\hline 30 & 774 \\
\hline 31 & 773 \\
\hline
\end{tabular}

26. Shutdowns Scheduled Over Next Six Months (Type, Date, Duration):

27. If Currently Shutdown, Estimated Startup Date:

Notes:

CUMULATIVE UNIT CAPACITY FACTOR (MDC NET) IS CALCULATED WITH A WEIGHTED AVERAGE. 


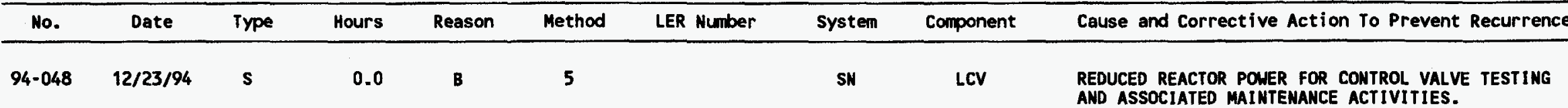
AND ASSOCIATED MAINTENANCE ACTIVITIES.

\begin{tabular}{|c|c|}
\hline TYPE & REASON \\
\hline $\begin{array}{l}\text { F: Forced } \\
\text { S: Scheduled }\end{array}$ & $\begin{array}{l}\text { A-Equipment Failure } \\
\text { B-Maintenance or Test } \\
\text { C-Refuel ing } \\
\text { D-Regulatory Restriction } \\
\text { E-Operator Iraining \& } \\
\text { License Examination } \\
\text { F-Administrative } \\
\text { G-Operational Error } \\
\text { H-Other }\end{array}$ \\
\hline
\end{tabular}

\begin{tabular}{l} 
METHOD \\
\hline 1-Manual \\
2-Manual Scram \\
3-Auto Scram \\
4-Cont inued \\
5-Reduced Load \\
9-0ther
\end{tabular}

\section{SYSTEM}

IEEE Standard

NUREG-0161 Exhibit $F$

\section{COMPONENT}

IEEE Standard

NUREG-0161 Exhibit $H$

G-Operational Error

H-Other 


\section{FACILITY DESCRIPTION}

\section{LOCATION}

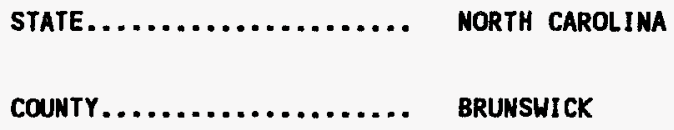

\section{UTILITY \& CONTRACTOR INFORMATION}

UTILITY

LICENSEE................ CAROLIMA POUER \& LIGHT CO.

CORPORATE ADDRESS........... P.O. BOX 1551

P.O. BOX 1551
RALEIGH, NORTH CAROLINA 27602

\section{CONTRACTOR}

ARCHITECT/ENGIMEER..........

UNITED ENG. \& CONSTRUCTORS

NUC STEAM SYS SUPPLIER

GENERAL ELECTRIC

CONSTRUCTOR.

BRONN \& ROOT

TURBINE SUPPLIER

GENERAL ELECTRIC

REGULATORY INFORMATION

IE REGION RESPONSIBLE......... 2

IE RESIDENT INSPECTOR.

CHARLES PATTERSON

LICENSING PROJ MAMAGER

PATRICK D. MILAMO

DOCKET NUMBER.

$50-324$

LICENSE \& DATE ISSUANCE 
1. Docket: $50-454$

OPERATING STATUS

2. Reporting Period: DECEMBER 1994

Outage + On-Line Hrs: 744.0

3. Utility Contact: R. COLGLAZIER (815) 234-5441 EXT. 2282

4. Licensed Thermal Power (MWt):

5. Nameplate Rating (Gross MWe):

6. Design Electrical Rating (Wet MWe):

7. Maximum Dependable Capacity (Gross MWe):

8. Maximum Dependable Capacity (Net MWe):

9. If Changes Occurred Above Since Last Report, Give Reasons:

10. Power Level To Which Restricted, If Any (Net MWe):

11. Reasons For Restrictions, If Any:

\begin{tabular}{|c|c|c|c|}
\hline & MONTH & YEAR & CUAMLATIVE \\
\hline 12. Report Period Hrs & 744.0 & $8,760.0$ & $81,457.0$ \\
\hline 13. Hours Reactor Critical & 454.4 & $7,174.8$ & $67,985.3$ \\
\hline 14. Rx Reserve Shtdwn Hrs & 0.0 & 0.0 & 38.0 \\
\hline 15. Hrs Generator On-Line & 447.7 & $7,138.1$ & $67,270.4$ \\
\hline 16. Unit Reserve Shtown Hrs & 0.0 & 0.0 & 0.0 \\
\hline 17. Gross Therm Ener (MWH) & $868,645.0$ & $20,981,890.0$ & $204,042,989.0$ \\
\hline 18. Gross Elec Ener (MUH) & $281,094.0$ & $7,159,737.0$ & $68,978,282.0$ \\
\hline 19. Net Elec Ener (MUH) & $258,271.0$ & $6,791,894.0$ & $65,355,223.0$ \\
\hline 20. Unit Service Factor & 60.2 & 81.5 & 82.6 \\
\hline 21. Unit Avail Factor & 60.2 & 81.5 & 82.6 \\
\hline 22. Unit Cap Factor (MDC Net) & 31.4 & 70.2 & 72.6 \\
\hline 23. Unit Cap Factor (DER Net) & 31.0 & 69.2 & 71.6 \\
\hline 24. Unit Forced Outage Rate & 39.8 & 4.0 & 2.6 \\
\hline 25. Forced Outage Hours & 296.3 & 296.3 & $1,794.6$ \\
\hline
\end{tabular}

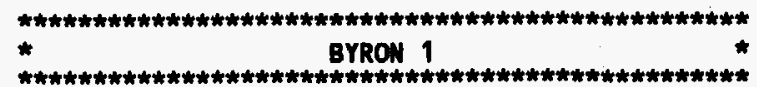

AVERAGe DAILY PONER LEVEL (Net MHe)

\begin{tabular}{ll} 
DAY & PONER \\
\hline 1 & 1082 \\
\hline 2 & 1077 \\
\hline 3 & 1026 \\
\hline 4 & -4 \\
\hline 5 & -14 \\
\hline 6 & -14 \\
\hline 7 & -14 \\
\hline 8 & -14 \\
\hline 9 & -14 \\
\hline 10 & -14 \\
\hline 11 & -14 \\
\hline 12 & -14 \\
\hline 13 & -14 \\
\hline 14 & -15 \\
\hline 15 & -14 \\
\hline
\end{tabular}

\begin{tabular}{ll} 
DAY & PONER \\
\hline 16 & 106 \\
\hline 17 & 214 \\
\hline 18 & 215 \\
\hline 19 & 211 \\
\hline 20 & 195 \\
\hline 21 & 211 \\
\hline 22 & 207 \\
\hline 23 & 273 \\
\hline 24 & 443 \\
\hline 25 & 445 \\
\hline 26 & 500 \\
\hline 27 & 542 \\
\hline 28 & 961 \\
\hline 29 & 1067 \\
\hline 30 & 1069 \\
\hline 31 & 1070 \\
\hline
\end{tabular}

26. Shutdowns Scheduled Over Mext Six Months (Type, Date, Duration):

27. If Currently Shutdown, Estimated Startup Date: Notes: 


\begin{tabular}{ccccccccc}
\hline No. Date & Type & Hours & Reason & Method & LER Number & System Component & Cause and Corrective Action To Prevent Recurrence \\
\hline 7 & $12 / 04 / 94$ & $F$ & 296.3 & & &
\end{tabular}

$7 \quad 12 / 04 / 94$

296.3

A

CD

CONDENSER TUBE FAILURE IN "D" WATERBOX.

\section{TYPE}

F: Forced

S: Scheduled
REASON

A-Equipment Failure

B-Maintenance or Test

C-Refueling

D-Regulatory Restriction

E-Operator Training \&

License Examination

F-Administrative

G-Operational Error

$H$-other

\begin{tabular}{l} 
METHCO \\
\hline 1-Manual \\
2-Manual Scram \\
3-Auto Scram \\
4-Cont inued \\
5-Reduced Load \\
9-0ther
\end{tabular}

SYSTEM

IEEE Standard

805-1984 and/or

NUREG-0161 Exhibit F

IEEE Standard

803A-1983 and/or

NUREG-0161 Exhibit H 


\section{FACILITY DESCRIPTION}

LOCATION

STATE.$\ldots \ldots \ldots \ldots \ldots \ldots \ldots$ ILLINOIS
COUNTY.$\ldots \ldots \ldots \ldots \ldots \ldots \ldots$ OGLE

DIST AND DIRECTION FROM

MEAREST POPULATION CTR..... 17 MI SH OF ROCKFORD, IL

TYPE OF REACTOR.

PUR

DATE INITIAL CRITICALITY...... FEBRUARY 02, 1985

DATE INITIAL ELECTRICITY...... MARCH 01, 1985

DATE COMMERCIAL OPERATE....... SEPTEMBER 16, 1985

CONDENSER COOLING METHOD...... CC HNDCT

CONDENSER COOLING WATER....... ROCK RIVER

ELECTRIC RELIABILITY

COUNCIL.................. MID-AMERICA INTERPOOL METWORK

\section{UTILITY \& CONTRACTOR INFORMATION}

\section{UTILITY}

LICENSEE.

CORPORATE ADDRESS

COMMONMEALTH EDISON CO.

1400 OPUS PL., OPUS WEST III SUITE 300

DOWNER'S GROVE, ILLINOIS 60515

\section{CONTRACTOR}

ARCHITECT/ENGIMEER .

NUC STEAM SYS SUPPLIER.

CONSTRUCTOR .

TURBINE SUPPLIER.

REGULATORY INFORMATION

IE REGION RESPONSIBLE......... 3

IE RESIDENT INSPECTOR.......... PETE PETERSON

LICENSING PROJ MANAGER......... GEORGE F. DICK

DOCKET MUMBER.............. $50-454$

LICENSE \& DATE ISSUANCE........ NPF 037, FEBRUARY 14, 1985 
1. Docket: $50-455$

OPERATING STATUS

2. Reporting Period: DECEMBER 1994 Outage + On-Line Hrs: 744.0

3. Utility Contact: R. COLGLAZIER (815) 234-5441 EXT. 2282

4. Licensed Thermal Power (MUt):

5. Nameplate Rating (Gross MHe):

6. Design Electrical Rating (Net MHe):

7. Maximum Dependable Capacity (Gross MWe):

8. Maximum Dependable Capacity (Het MWe):

9. If Changes Occurred Above Since Last Report, Give Reasons:

10. Power Level To Which Restricted, If Any (Wet MWe):

11. Reasons For Restrictions, If Any:

\begin{tabular}{|c|c|c|c|}
\hline & MONTH & YEAR & CUMULATIVE \\
\hline 12. Report Period Hrs & 744.0 & $8,760.0$ & $64,561,0$ \\
\hline 13. Hours Reactor Critical & 744.0 & $8,709.5$ & $56,515.4$ \\
\hline 14. Rx Reserve Shtdwn Hrs & 0.0 & $0=0$ & 0.0 \\
\hline 15. Hrs Generator On-Line & 744.0 & $8,704.2$ & $55,909.8$ \\
\hline 16. Unit Reserve Shtdwn Mrs & 0.0 & 0.0 & 0.0 \\
\hline 17. Gross Therm Ener (MUH) & $2,502,958.0$ & $28,911,971.0$ & $164,983,899.0$ \\
\hline 18. Gross Elec Ener (MUH) & $864,764.0$ & $9,950,910.0$ & $56,134,184.0$ \\
\hline 19. Net Elec Ener (MWH) & $820,665.0$ & $9,504,170.0$ & $53,287,397.0$ \\
\hline 20. Unit Service factor & 100.0 & 99.4 & 86.6 \\
\hline 21. Unit Avail Factor & 100.0 & 99.4 & 86.6 \\
\hline 22. Unit Cap Factor (MDC Net) & 99.8 & 98.2 & 74.7 \\
\hline 23. Unit Cap Factor (DER Net) & 98.5 & 96.9 & 73.7 \\
\hline 24. Unit Forced Outage Rate & 0.0 & 0.6 & 2.4 \\
\hline 25. Forced Outage Hours & 0.0 & 55.8 & $1,399.2$ \\
\hline
\end{tabular}

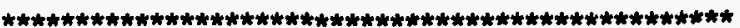

* BYRON 2 B

AVERAge daily poner leVel (Net MHe)

\begin{tabular}{ll} 
DAY & POWER \\
\hline 1 & 1105 \\
\hline 2 & 1104 \\
\hline 3 & 1101 \\
\hline 4 & 1089 \\
\hline 5 & 1099 \\
\hline 6 & 1006 \\
\hline 7 & 1101 \\
\hline 8 & 1103 \\
\hline 9 & 1100 \\
\hline 10 & 1106 \\
\hline 11 & 1104 \\
\hline 12 & 1099 \\
\hline 13 & 1097 \\
\hline 14 & 1096 \\
\hline 15 & 1095 \\
\hline
\end{tabular}

\begin{tabular}{ll} 
DAY & POWER \\
\hline 16 & 1100 \\
\hline 17 & 1112 \\
\hline 18 & 1116 \\
\hline 19 & 1115 \\
\hline 20 & 1110 \\
\hline 21 & 1109 \\
\hline 22 & 1108 \\
\hline 23 & 1106 \\
\hline 24 & 1113 \\
\hline 25 & 1109 \\
\hline 26 & 1105 \\
\hline 27 & 1103 \\
\hline 28 & 1112 \\
\hline 29 & 1117 \\
\hline 30 & 1119 \\
\hline 31 & 1120 \\
\hline
\end{tabular}

26. Shutdowns Scheduled Over Next Six Months (Type, Date, Duration):

27. If Currently Shutdown, Estimated Startup Date:

Notes: 
TYPE

F: Forced

S: Scheduled

\begin{tabular}{l} 
REASON \\
\hline A-Equipment Failure \\
B-Maintenance or Test \\
C-Refuel ing \\
D-Regulatory Restriction \\
E-Operator Training \& \\
License Examination \\
F-Administrative \\
G-Operational Error \\
H-Other
\end{tabular}

METHOD

1-Manual

2-Manual Scram

3-Auto Scram

4-Continued

5-Reduced Load

9-0ther

\section{SYSTEM}

IEEE Standard

805-1984 and/or
NUREG-0161 Exhibit F

\section{COMPONENT}

IEEE Standard

803A-1983 and/or

NUREG-0161 Exhibit 


\section{FACILITY DESCRIPTION}

\section{LOCATION}

STATE................ ILLINOIS
COUNTY.............. OGLE

\section{DIST AMD DIRECTION FROM}

MEAREST POPULATION CTR..... 17 MI SW OF ROCKFORD, IL

TYPE OF REACTOR

DATE INITIAL CRITICALITY...... JAMUARY 09, 1987

DATE INITIAL ELECTRICITY...... FEBRUARY 06, 1987

DATE COMMERCIAL OPERATE....... AUGUST 21, 1987

CONDENSER COOLING METHOD...... CCHNDCT

CONDENSER COOLING WATER....... ROCK RIVER

ELECTRIC RELIABILITY

COUNCIL................... MID-AMERICA INTERPOOL NETWORK

\section{UTILITY \& CONTRACTOR INFORMATION}

UTILITY

LICENSEE................ COMMONHEALTH EDISON CO.

CORPORATE ADDRESS.......... 1400 OPUS PL., OPUS WEST III SUITE 300 DOWER'S GROVE, ILLIMOIS 60515

\section{CONTRACTOR}

ARCHITECT/ENGINEER.

SARGENT \& LUNDY

NUC STEAM SYS SUPPLIER

\section{WESTINGHOUSE}

CONSTRUCTOR.

COMMONUEALTH EDISON

TURBINE SUPPLIER

WESTINGHOUSE

REGULATORY INFORMATION

IE REGION RESPONSIBLE......... 3

IE RESIDENT INSPECTOR......... PETE PETERSON

LICENSING PROJ MAMAGER......... GEORGE F. DICK

DOCKET NUMBER

$50-455$

LICENSE \& DATE ISSUANCE 


\section{Docket: $50-483$}

OPERATING STATUS

2. Reporting Period: DECEMBER 1994

Outage + On-Line Hrs:

744.0

3. Utility Contact: J. B. MCINVALE (314) 676-8247

4. Licensed Thermal Power (MUt):

5. Nameplate Rating (Gross MUe):

6. Design Electrical Rating (Net MWe):

7. Maximum Dependable Capacity (Gross MWe):

8. Maximm Dependable Capacity (Net MWe):

$\begin{array}{r}\hline 3565 \\ \hline 1236 \\ \hline 1171 \\ \hline 1232 \\ \hline 1115 \\ \hline\end{array}$

9. If Changes Occurred Above Since Last Report, Give Reasons:

10. Power Level To which Restricted, If Any (Net MWe):

11. Reasons For Restrictions, If Any:

\begin{tabular}{|c|c|c|c|}
\hline Report Period Hrs & $\begin{array}{l}\text { MONTH } \\
744.0\end{array}$ & $\begin{array}{l}\text { YEAR } \\
8,760.0\end{array}$ & $\begin{array}{l}\text { CUMULATIVE } \\
87,926.0\end{array}$ \\
\hline Hours Reactor Critical & 744.0 & $8,760.0$ & $77,697.7$ \\
\hline Rx Reserve Shtdwn Hrs & 0.0 & $\overline{0.0}$ & 0.0 \\
\hline Hrs Generator on-Line & 744.0 & $8,726.7$ & $76,281.8$ \\
\hline Unit Reserve Shtdun Hrs & 0.0 & 0.0 & 0.0 \\
\hline Gross Therm Ener (MUH) & $2,632,215.0$ & $30,691,681.0$ & $257,556,769.0$ \\
\hline Gross Elec Ener (MWH) & $906,918.0$ & $10,480,645.0$ & $87,851,768.0$ \\
\hline Net Elec Ener (MWH) & $866,630.0$ & $\overline{10,006,491.0}$ & $83,570,542.0$ \\
\hline Unit Service Factor & 100.0 & 99.6 & 86.8 \\
\hline Unit Avail Factor & 100.0 & 99.6 & 86.8 \\
\hline Unit Cap factor (MDC Net) & 104.5 & 102.4 & 85.2 \\
\hline Unit Cap Factor (DER Net) & 99.5 & 97.5 & 81.2 \\
\hline Init Forced Outage Rate & 0.0 & 0.0 & 2.3 \\
\hline orced Outage Hours & 0.0 & 0.0 & $1,775.6$ \\
\hline
\end{tabular}

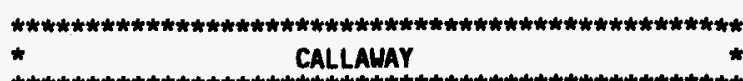

************) CALLAUAY

AVERAGe dAILY PONER LEVEL (Net MHe)

\begin{tabular}{ll} 
DAY & PONER \\
\hline 1 & 1163 \\
\hline 2 & 1157 \\
\hline 3 & 1162 \\
\hline 4 & 1160 \\
\hline 5 & 1164 \\
\hline 6 & 1166 \\
\hline 7 & 1166 \\
\hline 8 & 1167 \\
\hline 9 & 1163 \\
\hline 10 & 1170 \\
\hline 11 & 1170 \\
\hline 12 & 1169 \\
\hline 13 & 1171 \\
\hline 14 & 1168 \\
\hline 15 & 1167 \\
\hline
\end{tabular}

\begin{tabular}{cc} 
DAY & POWER \\
\hline 16 & 1156 \\
\hline 17 & 1164 \\
\hline 18 & 1166 \\
\hline 19 & 1166 \\
\hline 20 & 1167 \\
\hline 21 & 1170 \\
\hline 22 & 1165 \\
\hline 23 & 1165 \\
\hline 24 & 1169 \\
\hline 25 & 1169 \\
\hline 26 & 1167 \\
\hline 27 & 1159 \\
\hline 28 & 1144 \\
\hline 29 & 1167 \\
\hline 30 & 1167 \\
\hline 31 & 1168 \\
\hline
\end{tabular}

26. Shutdowns Scheduled Over Next six Months (Type, Date, Duration): REFUELING OUTAGE, MARCH 25, 1995, 56 DAYS.

27. If Currently Shutdown, Estimated Startup Date:

Notes: 


No. Date Type Hours Reason Method LER Number System Component Cause and Corrective Action To Prevent Recurrence

\begin{tabular}{l} 
TYPE \\
\hline F: Forced \\
S: Scheduled
\end{tabular}

REASON

A-Equipment Failure

C-Refuel ing

D-Regulatory Restriction

E-Operator Training \&

License Examination

F-Administrative

G-Operational Error

H-Other
METHOD

1-Manual

2-Manual Scram

3-Auto Scram

4-Cont inued

5-Reduced Load

9-other

\begin{tabular}{|c|c|}
\hline SYSTEM & COMPONENT \\
\hline $\begin{array}{l}\text { IEEE Standard } \\
805-1984 \text { and/or } \\
\text { MUREG-0161 Exh ibit F }\end{array}$ & $\begin{array}{l}\text { IEEE Standard } \\
803 \mathrm{~A}-1983 \text { and/or } \\
\text { MUREG-0161 Exhibit } \mathrm{H}\end{array}$ \\
\hline
\end{tabular}

Page 2-44 


\section{FACILITY DESCRIPTION}

LOCATION

STATE................... MISSOURI

COUNTY................ CALLAMAY

DIST AND DIRECTION FROM

MEAREST POPULATION CTR..... 10 MI SE OF FULTON, MO

TYPE OF REACTOR.

DATE INITIAL CRITICALITY...... OCTOBER 01, 1984

DATE INITIAL ELECTRICITY..... OCTOBER 24, 1984

DATE COMMERCIAL OPERATE.

CONDENSER COOLING METHOD

CONDENSER COOLING WATER.

ELECTRIC RELIABILITY

COUNCIL.
PUR

DECEMBER 19, 1984

COOLING TOWER

MISSOURI RIVER

MID-AMERICA INTERPOOL NETWORK

\section{UTILITY \& CONTRACTOR INFORMATION}

UT ILITY

LICENSEE.

CORPORATE ADDRESS

UNION ELECTRIC CO.

P.D. BOX 149

ST LOUIS, MISSOURI 63166

CONTRACTOR

ARCHITECT/ENGINEER......... BECHTEL

MUC STEAM SYS SUPPLIER...... WESTINGHOUSE

CONSTRUCTOR.............. DANIEL INTERNATIONAL

TURBINE SUPPLIER.......... GEMERAL ELECTRIC

\section{REGULATORY INFORMATION}

IE REGION RESPONSIBLE........ 3

IE RESIDENT INSPECTOR.

BRUCE BARTLETT

LICENSING PROJ MANAGER

RAYNARD WHARTON

DOCKET NUMBER............. 50-483

LICENSE \& DATE ISSUANCE.

NPF 030, OCTOBER 18, 1984 


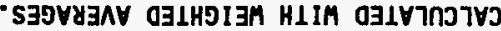

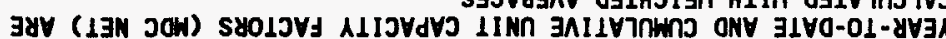

$$
\text { :sojoN }
$$

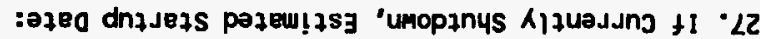

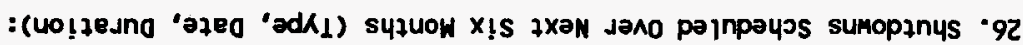

\begin{tabular}{ll}
\hline 998 & 15 \\
\hline 998 & $0 \varepsilon$ \\
\hline 298 & 62 \\
\hline 998 & 82 \\
\hline 998 & 22 \\
\hline 298 & 92 \\
\hline 298 & 52 \\
\hline 298 & 72 \\
\hline 298 & $\varepsilon 2$ \\
\hline 998 & 22 \\
\hline 998 & 12 \\
\hline 298 & 02 \\
\hline 298 & 61 \\
\hline 998 & 81 \\
\hline 998 & 21 \\
\hline 998 & 91 \\
$8990 d$ & 170
\end{tabular}

\begin{tabular}{|c|c|}
\hline 298 & Sl \\
\hline 598 & h \\
\hline 998 & $\boldsymbol{\varepsilon l}$ \\
\hline 998 & Zl \\
\hline 298 & $\boldsymbol{l l}$ \\
\hline 998 & ol \\
\hline 998 & 6 \\
\hline 998 & 8 \\
\hline 998 & $L$ \\
\hline 998 & 9 \\
\hline 998 & $\mathbf{s}$ \\
\hline 998 & 7 \\
\hline 998 & $\boldsymbol{\varepsilon}$ \\
\hline 598 & 2 \\
\hline 598 & $l$ \\
\hline yamod & Avo \\
\hline
\end{tabular}

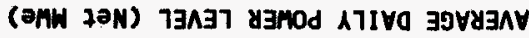

$* * * * * * * * * * * * * * * * * * * * * * * * * * * * * * * * * * * * * * * * * * * * * * * * * * *$

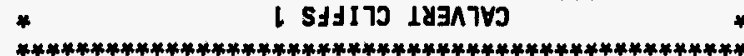

\begin{tabular}{|c|c|c|}
\hline$\varepsilon^{\circ} 00 L^{\prime}$ U & 9. slL & $0=0$ \\
\hline 6.8 & $2 \cdot 11$ & 0.0 \\
\hline$\varepsilon .99$ & 789 & 5.201 \\
\hline 6.29 & $\varepsilon \cdot 79$ & L. $\mathrm{EOL}$ \\
\hline 8.69 & 9.49 & 0.001 \\
\hline 8.69 & $9 \cdot 79$ & 0.001 \\
\hline 0.892'767'96 & $0^{\circ} 986^{\prime} 689^{\prime}$ ' & 0.86ฑ"לtด \\
\hline $0^{\circ} 919^{\prime} 0 \angle \varepsilon^{\prime} 101$ & $0^{\circ} 020^{\prime} \angle 68^{\prime} \Rightarrow$ & $0^{\circ} \angle 56^{\circ} 0 \angle 9$ \\
\hline $0^{\circ} 乏 68^{\prime} \angle 9 l^{\prime}$ 'SOE & $0^{\circ} 7 \angle 0^{\circ} \varepsilon 58^{\prime} \mathrm{hl}$ & $0^{\circ} L \angle l^{\prime} 900^{\prime} z$ \\
\hline 0.0 & 0.0 & 0.0 \\
\hline S.9ع2'0zl & $1.859^{\prime} 5$ & $0 \cdot ヤ タ<$ \\
\hline $7.610^{\prime} \mathrm{c}$ & 0.0 & 0.0 \\
\hline 9.088'221 & $20166^{\prime} 5$ & 0・サクく \\
\hline $0^{\circ} 192^{\prime} 2 \angle 1$ & $0.09 L^{\prime} 8$ & 0゙ヤクL \\
\hline כณโมทากพกว & צ४ $\exists \lambda$ & HLNOW \\
\hline
\end{tabular}

s.Jnoh abezno pəojof ' $\varsigma 2$ ajey a6ezno pajsos 7 ! un $\cdot 72$

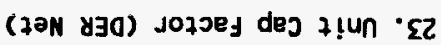
(7әN JOW) Jozoed I!eny tịn $\cdot 12$

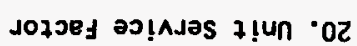

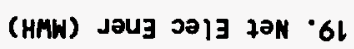

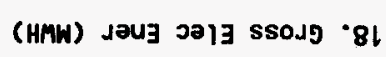
(HNW) JaUg سدaY1 ssojg $\cdot 21$ SJH UmpzyS әAJәsəy 7 ! un 91

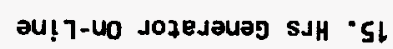
SJH Umpłys әAjasay $x y-7 l$ |

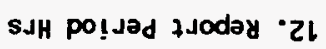

:Ruy +1 'suo!ta!jtsay jog suoseay ' IL

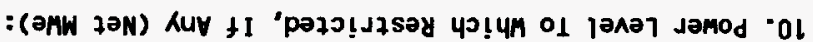

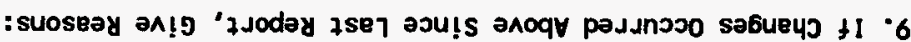

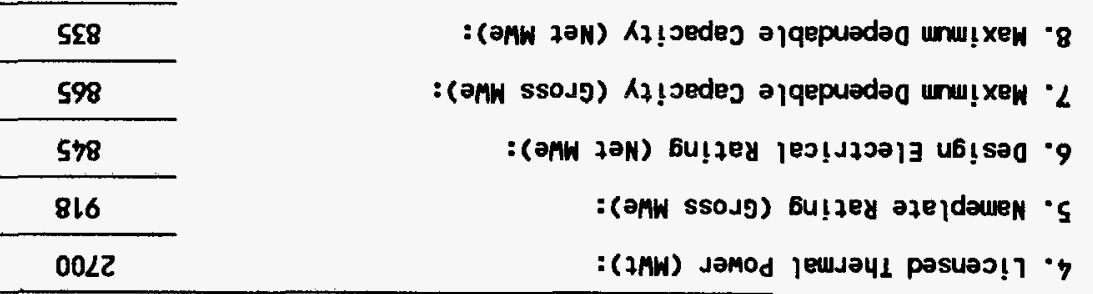

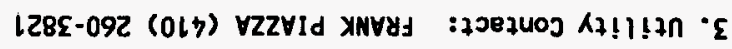

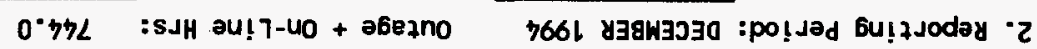

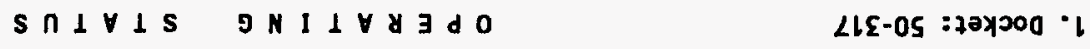




\begin{tabular}{|c|c|}
\hline TYPE & REASOH \\
\hline $\begin{array}{l}\text { F: Forced } \\
\text { S: Scheduled }\end{array}$ & $\begin{array}{l}\text { A-Equipment Failure } \\
\text { B-Maintenance or Test } \\
\text { C-Refuel ing } \\
\text { D-Regulatory Restriction } \\
\text { E-Operator Training \& } \\
\text { License Examination } \\
\text { F-Administrative } \\
\text { G-Operational Error } \\
\text { H-Other }\end{array}$ \\
\hline
\end{tabular}

\section{METHOD}

1-Manual

2-Manual Scram

3-Auto scram

4-Continued

5-Reduced Load

9-Other

\begin{tabular}{l} 
SYSTEM \\
\hline IEEE Standard \\
$805-1984$ and/or \\
MUREG-0161 Exhibit F
\end{tabular}

NUREG-0169 Exhibit $F$
CONPONENT

IEEE Standard

803A-1983 and/or

NUREG-0161 Exhibit H

H-other 


\section{FACILITY DESCRIPTION}

\section{LOCATION}

STATE.

COUNTY

DIST AND DIRECTION FROM

MEAREST POPULATION CTR.....

TYPE OF REACTOR.

DATE IMITIAL CRITICALITY...... OCTOBER 07, 1974

DATE INITIAL ELECTRICITY...... DECEMBER 30, 1974

DATE COMMERCIAL OPERATE....... MAY 08, 1975

CONDENSER COOLING METHOD...... ONCE THRU

CONDENSER COOLING HATER....... CHESAPEAKE BAY

ELECTRIC RELIABILITY

COUNCIL..............
MARYLAND

CALVERT

$40 \mathrm{MI} S \mathrm{~S}$ OF ANNAPOLIS, MD

PWR

MID-ATLANTIC AREA COUNCIL

\section{UTILITY \& CONTRACTOR INFORMATION}

UTILITY

LICENSEE.$\ldots \ldots \ldots \ldots \ldots \ldots$ BALtimORE GAS \& ELEC CO.

CORPORATE ADDRESS.

P.O. BOX 1475

BALTIMORE, MARYLAND 21203

CONTRACTOR

ARCH I TECT /ENGINEER .

BECHTEL

HUC STEAM SYS SUPPLIER...... COMBUSTION ENGIMEERING

CONSTRUCTOR

BECHTEL

TURBINE SUPPLIER

GENERAL ELECTRIC

\section{REGULATORY INFORMATION}

IE REGION RESPONSIBLE......... 1

IE RESIDENT INSPECTOR.

PETER R. HILSON

LICENSING PROJ MANAGER.

DANIEL G. MCDONALD

DOCKET MUMBER.

50-317

LICENSE \& DATE ISSUARCE........ DPR 053, JULY 31, 1974 


\section{Docket: $50-318$}

OPERATING STATUS

2. Reporting Period: DECEMBER 1994 Outage + On-Line Hrs: $\quad 744.0$

3. Utility Contact: FRANK PIAZZA (410) 260-3821

4. Licensed Thermal Power (MWt):

5. Nameplate Rating (Gross MWe):

6. Design Electrical Rating (Net MHe):

7. Maximum Dependable Capacity (Gross MWe):

8. Maximum Dependable Capacity (Net MWe):

9. If Changes Occurred Above Since Last Report, Give Reasons:

10. Power Level To thich Restricted, If Any (Net Mue):

11. Reasons For Restrictions, If Any:

\begin{tabular}{|c|c|c|c|}
\hline & MONTH & YEAR & CUMULATIVE \\
\hline 12. Report Period Hrs & 744.0 & $8,760.0$ & $155,616.0$ \\
\hline 13. Hours Reactor Critical & 744.0 & $8,000.2$ & $114,037.0$ \\
\hline 14. Rx Reserve Shtdun Hrs & 0.0 & 0.0 & $1,296.8$ \\
\hline 15. Hrs Generator On-Line & 744.0 & $7,927.1$ & $112,450.7$ \\
\hline 16. Unit Reserve Shtdun Hrs & 0.0 & 0.0 & $\overline{0.0}$ \\
\hline 17. Gross Therm Ener (MUH) & $2,000,640.0$ & $20,631,386.0$ & $287,496,929.0$ \\
\hline 18. Gross Elec Ener (MWH) & $671,503.0$ & $6,859,440.0$ & $95,015,309.0$ \\
\hline 19. Net Elec Ener (MWH) & $645,523.0$ & $6,578,476.0$ & $90,821,610.0$ \\
\hline 20. Unit Service Factor & 100.0 & 90.5 & 72.3 \\
\hline 21. Unit Avail Factor & 100.0 & 90.5 & 72.3 \\
\hline 22. Unit Cap Factor (MDC Net) & 103.3 & 89.8 & 70.7 \\
\hline 23. Unit Cap Factor (DER Net) & 102.7 & 88.9 & 69.1 \\
\hline 24. Unit Forced Outage Rate & 0.0 & 7.9 & 5.8 \\
\hline 25. Forced Outage Hours & 0.0 & 684.1 & $6,977.6$ \\
\hline
\end{tabular}

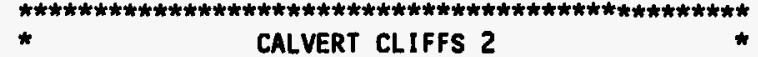

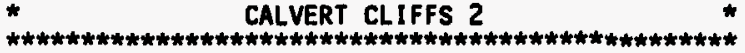

AVERAGE DAILY POWER LEVEL (Net MWe)

\begin{tabular}{cc}
\hline DAY & PONER \\
\hline 1 & 868 \\
\hline 2 & 868 \\
\hline 3 & 868 \\
\hline 4 & 869 \\
\hline 5 & 868 \\
\hline 6 & 868 \\
\hline 7 & 868 \\
\hline 8 & 868 \\
\hline 9 & 868 \\
\hline 10 & 868 \\
\hline 11 & 868 \\
\hline 12 & 868 \\
\hline 13 & 867 \\
\hline 14 & 868 \\
\hline 15 & 868 \\
\hline
\end{tabular}

\begin{tabular}{cc} 
DAY & POWER \\
\hline 16 & 868 \\
\hline 17 & 867 \\
\hline 18 & 867 \\
\hline 19 & 867 \\
\hline 20 & 867 \\
\hline 21 & 868 \\
\hline 22 & 867 \\
\hline 23 & 868 \\
\hline 24 & 867 \\
\hline 25 & 868 \\
\hline 26 & 868 \\
\hline 27 & 867 \\
\hline 28 & 868 \\
\hline 29 & 867 \\
\hline 30 & 866 \\
\hline 31 & 868 \\
\hline
\end{tabular}

26. Shutdowns Scheduled Over Next six Months (Type, Date, Duration): REFUELING OUTAGE, MARCH 17, 1995, 65 DAYS.

27. If Currently Shutdown, Estimated Startup Date: Notes:

YEAR-TO-DATE AND CUMULATIVE UNIT CAPACITY FACTORS (MDC NET) ARE CALCULATED WITH WEIGHTED AVERAGES. 


\section{A-Equipment Failure} B-Maintenance or Test C-Refuel ing

D-Regulatory Restriction

E-operator Training \&

License Examination

F-Adninistrative

G-operational Error

H-Other

\section{METHOD}

1-Manual

2-Manual Scram

3-Auto Scram

4-Cont inued

5-Reduced Load

9-Other

\section{SYSTEM}

IEEE Standard

805-1984 and/or

NUREG-0161 Exhibit
COMPONENT

IEEE Standard

803A-1983 and/or

NUREG-0161 Exhibit 


\section{FACILITY DESCRIPTION}

\section{LOCATION}

STATE

COUNTY

DIST AND DIRECTION FROM

MEAREST POPULATION CTR..... 40 MI S OF AMHAPOLIS, MD

TYPE OF REACTOR.

PUR

DATE INITIAL CRITICALITY...... NOVEMBER 30, 1976

DATE INITIAL ELECTRICITY...... DECEMBER 07, 1976

DATE COMMERCIAL OPERATE....... APRIL 01, 1977

CONDENSER COOLING METHOO...... ONCE THRU

CONDENSER COOLING WATER...... CHESAPEAKE BAY

ELECTRIC RELIABILITY

COUNCIL................... MID-ATLANTIC AREA COUNCIL

\section{UTILITY \& CONTRACTOR INFORMATION}

UTILITY

LICENSEE.

CORPORATE ADDRESS.

BALTIMORE GAS \& ELEC CO.

P.0. BOX 1475

BALTIMORE, MARYLAND 21203

CONTRACTOR

ARCHITECT/ENGINEER.......... BECHTEL

MUC STEAM SYS SUPPLIER...... COMBUSTION ENGIMEERING

CONSTRUCTOR

BECHTEL

TURBINE SUPPLIER

MEST INGHOUSE

\section{REGULATORY INFORMATION}

IE REGION RESPONSIBLE.

1

IE RESIDENT INSPECTOR

PETER R. WILSON

LICENSING PROJ MANAGER.

DANIEL G. MCDONALD

DOCKET NUMBER.

$50-318$

LICENSE \& DATE ISSUANCE.

DPR 069, NOVEMBER 30, 1976 
1. Docket: $50-413$

OPERATING STATUS

2. Reporting Period: DECEMBER 1994

Outage + On-Line Hrs: 744.0

3. Utility Contact: R. A. WILLIAMS (704) 382-5346

4. Licensed Thermal Power (MUt):

5. Nameplate Rating (Gross MHe):

6. Design Electrical Rating (Net MHe):

7. Maximum Dependable Capacity (Gross MWe):

8. Maximum Dependable Capacity (Net MUe):

9. If Changes Occurred Above Since Last Report, Give Reasons:

10. Power Level To Which Restricted, If Any (Net MHe):

11. Reasons For Restrictions, If Any:

\begin{tabular}{|c|c|c|c|}
\hline & MONTH & YEAR & CUMULATIVE \\
\hline 12. Report Period Hrs & 744.0 & $8,760.0$ & $83,353.0$ \\
\hline 13. Hours Reactor Critical & 744.0 & $8,733.6$ & $64,511.7$ \\
\hline 14. Rx Reserve Shtdun Hrs & 0.0 & 0.0 & 0.0 \\
\hline 15. Hrs Generator On-Line & 744.0 & $8,722.1$ & $63,375.8$ \\
\hline 16. Unit Reserve Shtdwn Hrs & 0.0 & 0.0 & 0.0 \\
\hline 17. Gross Therm Ener (MUH) & $2,497,634.0$ & $28,900,895.0$ & $206,143,431.0$ \\
\hline 18. Gross Elec Ener (MUH) & $900,745.0$ & $10,319,615.0$ & $72,683,750.0$ \\
\hline 19. Net Elec Ener (MUH) & $855,397.0$ & $9,778,833.0$ & $68,323,462.0$ \\
\hline 20. Unit Service Factor & 100.0 & 99.6 & 76.0 \\
\hline 21. Unit Avail Factor & 100.0 & 99.6 & 76.0 \\
\hline 22. Unit Cap Factor (MDC Net) & 101.8 & 98.9 & 72.3 \\
\hline 23. Unit Cap Factor (DER Net) & 100.4 & 97.5 & 71.6 \\
\hline 24. Unit Forced Outage Rate & 0.0 & 0.4 & 9.0 \\
\hline 25. Forced Outage Hours & 0.0 & 36.7 & $6,305.2$ \\
\hline
\end{tabular}

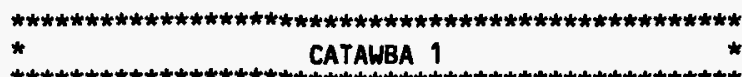

AVERAGE DAILY POWER LEVEL (Net MWe)

\begin{tabular}{ll} 
DAY & POWER \\
\hline 1 & 1156 \\
\hline 2 & 1156 \\
\hline 3 & 1121 \\
\hline 4 & 1145 \\
\hline 5 & 1143 \\
\hline 6 & 1147 \\
\hline 7 & 1147 \\
\hline 8 & 1151 \\
\hline 9 & 1154 \\
\hline 10 & 1151 \\
\hline 11 & 1152 \\
\hline 12 & 1155 \\
\hline 13 & 1155 \\
\hline 14 & 1153 \\
\hline 15 & 1151 \\
\hline
\end{tabular}

\begin{tabular}{ll} 
DAY & POWER \\
\hline 16 & 1153 \\
\hline 17 & 1152 \\
\hline 18 & 1152 \\
\hline 19 & 1154 \\
\hline 20 & 1153 \\
\hline 21 & 1152 \\
\hline 22 & 1149 \\
\hline 23 & 1149 \\
\hline 24 & 1151 \\
\hline 25 & 1152 \\
\hline 26 & 1151 \\
\hline 27 & 1152 \\
\hline 28 & 1152 \\
\hline 29 & 1151 \\
\hline 30 & 1152 \\
\hline 31 & 1132 \\
\hline
\end{tabular}

26. Shutdowns Scheduled Over Next Six Months (Type, Date, Duration): REFUELING OUTAGE, FEBRUARY 11, 1995, 53 DAYS.

27. If Currently Shutdown, Estimated Startup Date:

Notes:

CUMULATIVE UNIT CAPACITY FACTOR (MDC NET) IS CALCULATED WITH A WEIGHTED AVERAGE. 
TYPE

F: Forced

S: Scheduled

\section{REASON}

A-Equipment Failure B-Maintenance or Test C-Refueling

D-Regulatory Restriction

E-Operator Training \&

License Examination

F-Administrative

G-Operational Error

H-Other

\section{SYSTEM}

METHOD

1-Manual

2-Manual Scram

3-Auto Scram

4-Continued

5-Reduced Load

9-0ther

\section{COMPONENT}

IEEE Standard

IEEE Standard

805-1984 and/or
MUREG-0161 Exhibit

NUREG-0161 Exhibit H 


\section{FACILITY DESCRIPTION}

\section{LOCATION}

STATE.

COUNTY

DIST AND DIRECTION FROM

MEAREST POPULATION CTR..... 6 MI NNH OF ROCK HILL, SC

TYPE OF REACTOR

PUR

DATE INITIAL CRITICALITY...... JANUARY 07, 1985

DATE INITIAL ELECTRICITY...... JAMUARY 22, 1985

DATE COMMERCIAL OPERATE....... JUNE 29, 1985

CONDENSER COOLING METHOD...... MDCT

CONDENSER COOLING WATER....... LAKE WYLIE

ELECTRIC RELIABILITY

COUNCIL.................. SOUTHEASTERN ELECTRIC

RELIABILITY COUNCIL

\section{UTILITY \& CONTRACTOR INFORMATION}

UTILITY

LICENSEE.

DUKE PONER CO.

CORPORATE ADORESS

422 SOUTH CHURCH STREET

CHARLOTTE, NORTH CAROLINA 28242

\section{CONTRACTOR}

ARCHITECT/ENGINEER

DUKE POWER

NUC STEAM SYS SUPPLIER..... MESTINGHOUSE

CONSTRUCTOR

DUKE POWER

TURBINE SUPPLIER

GENERAL ELECTRIC

\section{REGULATORY INFORMATION}

IE REGION RESPONSIBLE......... 2

IE RESIDENT INSPECTOR......... RICHARD FREUDENBERGER

LICENSING PROJ MANAGER......... ROBERT E. MARTIN

DOCKET NUMBER.............. 50-413

LICENSE \& DATE ISSUANCE........ MPF 035, JANUARY 17, 1985 
1. Docket: $50-414$

OPERATING STATUS

2. Reporting Period: DECEMBER 1994

Outage + On-Line Hrs: 744.0

3. Utility Contact: R. A. HILLIAMS (704) 382-5346

4. Licensed Thermal Power (MUt):

5. Nameplate Rating (Gross MHe):

6. Design Electrical Rating (Net MWe):

7. Maximum Dependable Capacity (Gross MWe):

8. Maximum Dependable Capacity (Net MWe):

9. If Changes Occurred Above Since Last Report, Give Reasons:

10. Power Level To thich Restricted, If Any (Net MHe):

11. Reasons For Restrictions, If Any:

\begin{tabular}{|c|c|c|c|}
\hline & MONTH & YEAR & CUMULATIVE \\
\hline 12. Report Period Hrs & 744.0 & $8,760.0$ & $73,369.0$ \\
\hline 13. Hours Reactor Critical & 744.0 & $7,068.5$ & $57,009.3$ \\
\hline 14. Rx Reserve Shtdwn Hrs & 0.0 & 0.0 & 0.0 \\
\hline 15. Hrs Generator On-Line & 744.0 & $6,982.0$ & $56,061.7$ \\
\hline 16. Unit Reserve Shtdun Hrs & 0.0 & 0.0 & 0.0 \\
\hline 17. Gross Therm Ener (MUH) & $2,527,428.0$ & $22,809,844.0$ & $179,698,883.0$ \\
\hline 18. Gross Elec Ener (MUH) & $901,752.0$ & $8,122,121.0$ & $63,699,380.0$ \\
\hline 19. Net Elec Ener (MWH) & $856,984.0$ & $7,675,496.0$ & $59,965,553.0$ \\
\hline 20. Unit Service Factor & 100.0 & 79.7 & 76.4 \\
\hline 21. Unit Avail Factor & 100.0 & 79.7 & 76.4 \\
\hline 22. Unit Cap Factor (MDC Net) & 102.0 & 77.6 & 72.2 \\
\hline 23. Unit Cap Factor (DER Net) & 100.6 & 76.5 & 71.4 \\
\hline 24. Unit Forced Outage Rate & 0.0 & 2.7 & 8.9 \\
\hline 25. Forced Outage Hours & 0.0 & 196.6 & $5,457.8$ \\
\hline
\end{tabular}

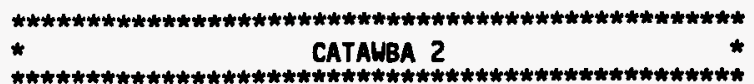

AVERAGE DAILY POWER LEVEL (Net MUe)

\begin{tabular}{ll} 
DAY & PONER \\
\hline 1 & 1154 \\
\hline 2 & 1154 \\
\hline 3 & 1152 \\
\hline 4 & 1146 \\
\hline 5 & 1142 \\
\hline 6 & 1148 \\
\hline 7 & 1146 \\
\hline 8 & 1150 \\
\hline 9 & 1153 \\
\hline 10 & 1150 \\
\hline 11 & 1153 \\
\hline 12 & 1155 \\
\hline 13 & 1154 \\
\hline 14 & 1153 \\
\hline 15 & 1152 \\
\hline
\end{tabular}

\begin{tabular}{ll} 
DAY & PONER \\
\hline 16 & 1153 \\
\hline 17 & 1135 \\
\hline 18 & 1152 \\
\hline 19 & 1155 \\
\hline 20 & 1155 \\
\hline 21 & 1155 \\
\hline 22 & 1153 \\
\hline 23 & 1153 \\
\hline 24 & 1154 \\
\hline 25 & 1155 \\
\hline 26 & 1154 \\
\hline 27 & 1155 \\
\hline 28 & 1154 \\
\hline 29 & 1154 \\
\hline 30 & 1155 \\
\hline 31 & 1155 \\
\hline
\end{tabular}

26. Shutdowns Scheduled Over Next Six Months (Type, Date, Duration):

27. If Currently Shutdown, Estimated Startup Date:

Notes:

CUMULATIVE UNIT CAPACITY FACTOR (MDC MET) IS CALCULATED WITH A WEIGHTED AVERAGE. 


\begin{tabular}{|c|c|}
\hline TYPE & REASON \\
\hline $\begin{array}{l}\text { F: Forced } \\
\text { S: Scheduled }\end{array}$ & $\begin{array}{l}\text { A-Equipment Failure } \\
\text { B-Maintenance or Test } \\
\text { C-Refuel ing } \\
\text { D-Regulatory Restriction } \\
\text { E-Operator Training \& } \\
\text { License Examination } \\
\text { F-Administrative } \\
\text { G-Operational Error } \\
\text { H-Other }\end{array}$ \\
\hline
\end{tabular}

\begin{tabular}{l} 
METHOD \\
\hline 1-Manual \\
2-Manual Scram \\
3-Auto Scram \\
4-Cont inued \\
5-Reduced Load \\
9-0ther
\end{tabular}

\section{SYSTEM}

IEEE Standard

805-1984 and/or

NUREG-0161 Exhibit $F$
COMPONENT

IEEE Standard

803A-1983 and/or

NUREG-0161 Exhibit H

G-Operational Error 


\section{FACILITY DESCRIPTION}

\section{LOCATION}

STATE.

COUNTY.

SOUTH CAROLINA

YORK

DIST AND DIRECTION FROM

MEAREST POPULATION CTR..... 6 MI NNH OF ROCK HILL, SC

TYPE OF REACTOR.

PUR

DATE InITIAL CRITICALITY...... MAY 08, 1986

DATE INITIAL ELECTRICITY...... MAY 18, 1986

DATE COMMERCIAL OPERATE....... AUgust 19,1986

CONDENSER COOLING METHOD...... MDCT

CONDENSER COOLING WATER...... LAKE WYLIE

ELECTRIC RELIABILITY

COUNCIL................. SOUTHEASTERN ELECTRIC

RELIABILITY COUNCIL

\section{UTILITY \& CONTRACTOR INFORMATION}

\section{UTILITY}

LICENSEE

CORPORATE ADDRESS

\section{CONTRACTOR}

ARCHI TECT/ENGINEER

NUC STEAM SYS SUPPLIER.

CONSTRUCTOR

TURBINE SUPPLIER

\section{REGULATORY INFORMATION}

IE REGION RESPONSIBLE.........

IE RESIDENT INSPECTOR.......... RICHARD FREUDENBERGER

LICENSING PROJ MANAGER......... ROBERT E. MARTIN

DOCKET NUMBER............. $50-414$

LICENSE \& DATE ISSUANCE....... NPF 052, MAY 15, 1986 
1. Docket: $50-461$

OPERATING STATUS

2. Reporting Period: DECEMBER 1994

Outage + On-Line Hrs: 744.0

3. Utility Contact: M. C. HOLLON (217) 935-8881 EXT. 3537

4. Licensed Thermal Power (MWt):

5. Nameplate Rating (Gross MMe):

6. Design Electrical Rating (Net MWe):

7. Maximum Dependable Capacity (Gross MUe):

8. Maximum Dependable Capacity (Het MWe):

\begin{tabular}{c}
744.0 \\
\hline 2894 \\
\hline 985 \\
\hline 933 \\
\hline 973 \\
\hline 930
\end{tabular}

9. If Changes Occurred Above Since Last Report, Give Reasons:

10. Power Level To Which Restricted, If Any (Net MWe):

11. Reasons for Restrictions, If Any:

MONTH YEAR CUMULATIVE

\section{Report Period Hrs}

13. Hours Reactor Critical

14. RX Reserve Shtoiwn Hirs

15. Hrs Generator On-Line

16. Unit Reserve Shtown Hrs

17. Gross Therm Ener (MUH)

18. Gross Elec Ener (MWH)

19. Net Elec Ener (MWH)

20. Unit Service Factor

21. Unit Avail Factor

22. Unit Cap factor (MDC Net)

23. Unit Cap Factor (DER Net)

24. Unit Forced Outage Rate

25. Forced Outage Hours

\begin{tabular}{|c|c|c|}
\hline 744.0 & $8,760.0$ & $62,266.0$ \\
\hline 744.0 & $8,308.1$ & $45, \pi 51.7$ \\
\hline 0.0 & 0.0 & 0.0 \\
\hline 744.0 & $8,218.3$ & $44,424.4$ \\
\hline 0.0 & 0.0 & 4.0 \\
\hline $2,155,951.0$ & $23,408,863.0$ & $118,466,198.0$ \\
\hline $712,406.0$ & $7,738,087.0$ & $39,156,951.0$ \\
\hline $684,494.0$ & $7,410,335.0$ & $37,276,157.0$ \\
\hline 100.0 & 93.8 & 71.3 \\
\hline 100.0 & 93.8 & 71.4 \\
\hline 98.9 & 91.0 & 64.4 \\
\hline 98.6 & 90.7 & 64.2 \\
\hline 0.0 & 0.2 & 9.2 \\
\hline 0.0 & 16.3 & $4,523.7$ \\
\hline
\end{tabular}

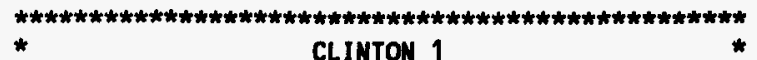

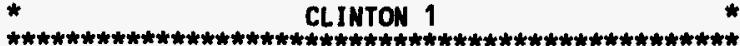

AVERAGE DAILY POWER LEVEL (Net MWe)

\begin{tabular}{ll} 
DAY & PONER \\
\hline 1 & 923 \\
\hline 2 & 925 \\
\hline 3 & 926 \\
\hline 4 & 926 \\
\hline 5 & 925 \\
\hline 6 & 925 \\
\hline 7 & 924 \\
\hline 8 & 924 \\
\hline 9 & 923 \\
\hline 10 & 923 \\
\hline 11 & 904 \\
\hline 12 & 904 \\
\hline 13 & 920 \\
\hline 14 & 920 \\
\hline 15 & 901 \\
\hline
\end{tabular}

\begin{tabular}{cc} 
DAY & PONER \\
16 & 921 \\
\hline 17 & 924 \\
\hline 18 & 920 \\
\hline 19 & 921 \\
\hline 20 & 921 \\
\hline 21 & 922 \\
\hline 22 & 923 \\
\hline 23 & 923 \\
\hline 24 & 918 \\
\hline 25 & 918 \\
\hline 26 & 925 \\
\hline 27 & 919 \\
\hline 28 & 910 \\
\hline 29 & 917 \\
\hline 30 & 918 \\
\hline 31 & 923 \\
\hline
\end{tabular}

26. Shutdowns Scheduled Over Mext Six Months (Type, Date, Duration):

REFUELING OUTAGE, MARCH 12, 1995, 50 DAYS.

27. If Currently Shutdown, Estimated Startup Date:

Notes: 


\begin{tabular}{|c|c|}
\hline TYPE & REASON \\
\hline $\begin{array}{l}\text { F: Forced } \\
\text { s: Scheduled }\end{array}$ & $\begin{array}{l}\text { A-Equipment Failure } \\
\text { B-Maintenance or Test } \\
\text { C-Refuel ing } \\
\text { D-Regulatory Restriction } \\
\text { E-Operator Training \& } \\
\text { License Examination } \\
\text { F-Administrat ive } \\
\text { G-Operat ional Error } \\
\text { H-Other }\end{array}$ \\
\hline
\end{tabular}

\begin{tabular}{l} 
METHOD \\
\hline 1-Manual Scram \\
2-Manual Scram \\
3-Auto Scram \\
4-Cont inued \\
5-Reduced Load \\
9-0ther
\end{tabular}

SYSTEN

IEEE Standard

805-1984 and/or

NUREG-0161 Exhibit $F$
COMPONENT

IEEE Standard

803A-1983 and/or

NUREG-0161 Exhibit H

F-Administrat ive

H-Other 


\section{FACILITY DESCRIPTION}

\section{LOCATION}

STATE................. ILLINOIS
COUNTY............... DEHITT

\section{DIST AND DIRECTION FRO}

MEAREST POPUI

TYPE OF REACTOR............. B B

DATE INITIAL CRITICALITY....... FEBRUARY 27, 1987

DATE INITIAL ELECTRICITY...... APRIL 24, 1987

DATE COMMERCIAL OPERATE........ NOVEMBER 24, 1987

CONDENSER COOLING METHOD...... ONCE THRU

CONDENSER COOLING WATER....... SALT CREEK

ELECTRIC RELIABILITY

COUNCIL................... MID-AMERICA INTERPOOL NETHORK
UTILITY \& CONTRACTOR INFORMATION

\section{UTILITY}

LICENSEE.................. ILLINOIS PONER Co.

CORPORATE ADDRESS.

500 SOUTH 27TH STREET

DECATUR, ILLINOIS 62525

\section{CONTRACTOR \\ ARCHITECT/ENGINEER.......... SARGENT \& LUNDY \\ NUC STEAM SYS SUPPLIER...... GEMERAL ELECTRIC \\ CONSTRUCTOR.............. BALDWIN ASSOCIATES \\ TURBINE SUPPLIER........... GENERAL ELECTRIC}

REGULATORY INFORMATION

IE REGION RESPONSIBLE........ 3

IE RESIDENT INSPECTOR.

MICHAEL MILLER

LICENSING PROJ MANAGER.

DOUGLAS V. PICKETT

DOCKET NUMBER

$50-461$

LICENSE \& DATE ISSUANCE

NPF 062, OCTOBER 09, 1987 
1. Docket: $50-445$

OPERATING STATUS

2. Reporting Period: DECEMBER 1994

Outage + On-Line Hrs:

744.0

3. Utility Contact: JANET HUGHES (817) 897-5331

4. Licensed Thermal Power (MHt):

5. Nameplate Rating (Gross MWe):

6. Design Electrical Rating (Net MWe):

7. Maximum Dependable Capacity (Gross MWe):

8. Maximum Dependable Capacity (Net MHe):

9. If Changes Occurred Above Since Last Report, Give Reasons:

10. Power Level To thich Restricted, If Any (Net MWe):

11. Reasons For Restrictions, If Any:

\begin{tabular}{|c|c|c|c|}
\hline & MONTH & YEAR & CUMULATIVE \\
\hline 12. Report Period Hrs & 744.0 & $8,760.0$ & $38,441.0$ \\
\hline 13. Hours Reactor Critical & 732.0 & $8,674.0$ & $31,213.3$ \\
\hline 14. Rx Reserve Shtcdun Hrs & 12.0 & 86.0 & $2,439.8$ \\
\hline 15. Hrs Generator on-Line & 715.0 & $8,653.0$ & $30,745.2$ \\
\hline 16. Unit Reserve Shtdwn Hrs & 0.0 & 0.0 & 0.0 \\
\hline 17. Gross Therm Ener (MUH) & $2,358,420.0$ & $29,185,302.0$ & $98,935,107.6$ \\
\hline 18. Gross Elec Ener (MWH) & $789,257.0$ & $9,761,370.0$ & $32,896,627.0$ \\
\hline 19. Net Elec Ener (MHH) & $755,700.0$ & $9,367,596.0$ & $31,363,447.0$ \\
\hline 20. Unit Service Factor & 96.1 & 98.8 & 80.0 \\
\hline 21. Unit Avail Factor & 96.1 & 98.8 & 80.0 \\
\hline 22. Unit Cap Factor (MDC Net) & 88.3 & 93.0 & 70.9 \\
\hline 23. Unit Cap Factor (DER Net) & 88.3 & 93.0 & 70.9 \\
\hline 24. Unit Forced Outage Rate & 3.9 & 1.2 & 4.8 \\
\hline 25. Forced Outage Hours & 29.0 & 107.0 & $1,539.7$ \\
\hline
\end{tabular}

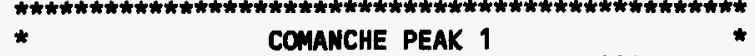

COMANCHE PEAK 1 ,

AVERAGE DAILY POWER LEVEL (Net MHe)

\begin{tabular}{lc} 
DAY & PONER \\
\hline 1 & 0 \\
\hline 2 & 52 \\
\hline 3 & 589 \\
\hline 4 & 1085 \\
\hline 5 & 1103 \\
\hline 6 & 1104 \\
\hline 7 & 1104 \\
\hline 8 & 1105 \\
\hline 9 & 1104 \\
\hline 10 & 1104 \\
\hline 11 & 1104 \\
\hline 12 & 1103 \\
\hline 13 & 1103 \\
\hline 14 & 1104 \\
\hline 15 & 1103 \\
\hline
\end{tabular}

\begin{tabular}{ll} 
DAY & PONER \\
\hline 16 & 1103 \\
\hline 17 & 1103 \\
\hline 18 & 1102 \\
\hline 19 & 1103 \\
\hline 20 & 1102 \\
\hline 21 & 1102 \\
\hline 22 & 1102 \\
\hline 23 & 1101 \\
\hline 24 & 1102 \\
\hline 25 & 1102 \\
\hline 26 & 1102 \\
\hline 27 & 1103 \\
\hline 28 & 1103 \\
\hline 29 & 1103 \\
\hline 30 & 1102 \\
\hline 31 & 1103 \\
\hline
\end{tabular}

26. Shutdowns Scheduled Over Mext Six Months (Type, Date, Duration): REFUELING OUTAGE, MARCH 2, 1995, 63 DAYS.

27. If Currently shutdown, Est imated Startup Date:

Notes: 


\begin{tabular}{|c|c|c|c|c|c|c|c|c|c|}
\hline No. & Date & Type & Hours & Reason & Method & LER Number & System & Component & Cause and Corrective Action To Prevent Recurrence \\
\hline 8 & $11 / 29 / 94$ & $\mathbf{F}$ & 19.0 & A & 4 & 94006 & & & $\begin{array}{l}\text { CONTINUED OUTAGE DUE TO REACTOR/TURBINE TRIP DUE } \\
\text { TO GENERATOR PRIMARY WATER STATOR LOW FLOW } \\
\text { SIGNAL. }\end{array}$ \\
\hline 9 & $12 / 02 / 94$ & $\mathbf{F}$ & 10.0 & A & 1 & & & & TURBINE TRIP DUE TO GENERATOR LOCKOUT ACTUATION. \\
\hline
\end{tabular}

\begin{tabular}{l} 
TYPE \\
\hline F: Forced \\
S: Scheduled
\end{tabular}

\section{REASON}

A-Equipment Failure

B-Maintenance or Test

C-Refuel ing

D-Regulatory Restriction

E-Operator Training \&

License Examination

F-Administrative

G-Operational Error

H-other

\begin{tabular}{l} 
METHOD \\
\hline 1-Manual \\
2-Manual Scram \\
3-Auto Scram \\
4-Continued \\
5-Reduced Losd \\
9-0ther
\end{tabular}

SYSTEM

IEEE Standard

805-1984 and/or

NUREG-0161 Exhibit F
COMPONENT

IEEE Standard

803A-1983 and/or

NUREG-0161 Exhibit H 


\section{FACILITY DESCRIPTION}

LOCATION

STATE.................. TEXAS
COUNTY................. SONERVELLE

DIST AND DIRECTION FROM

NEAREST POPULATION CTR.

TYPE OF REACTOR

DATE INITIAL CRITICALITY

DATE INITIAL ELECTRICITY

DATE COMMERCIAL OPERATE........ AUGUST 13, 1990

CONDENSER COOLING METHOD...... CC ART

CONDENSER COOLING WATER....... SQUAH CREEK RES

ELECTRIC RELIABILITY

COUNCIL................. ELECTRIC RELIABILITY COUNCIL OF TEXAS

\section{UTILITY \& CONTRACTOR INFORMATION}

UTILITY

LICENSEE.

CORPORATE ADDRESS.

TEXAS UTILITIES ELECTRIC CO.

400 N. OLIVE STREET

DALLAS, TEXAS 75201

\section{CONTRACTOR}

ARCHI TECT/ENGINEER

STONE \& MEBSTER

NUC STEAM SYS SUPPLIER...... WESTINGHOUSE

CONSTRUCTOR............... BROWN \& ROOT

TURBINE SUPPLIER........... ALLIS-CHALMERS

REGULATORY INFORMATION

IE REGION RESPONSIBLE......... 4

IE RESIDENT INSPECTOR......... ANTHONY GODY, JR.

LICENSING PROJ MANAGER......... THOMAS A. BERGMAN

DOCKET MUMBER .

$50-445$

LICENSE \& DATE ISSUANCE.

NPF-087, APRIL 16, 1990 
1. Docket: $50-446$

OPERATING STATUS

2. Reporting Period: DECEMBER 1994

Outage + On-Line Hrs: $\quad 744.0$

3. Utility Contact: JANET HUGHES (817) 897-5331

4. Licensed Thermal Power (MWt):

5. Nameplate Rating (Gross MWe):

6. Design Electrical Rating (Net MHe):

7. Maximum Dependable Capacity (Gross MWe):

8. Maximum Dependable Capacity (Net MHe):

9. If Changes Occurred Above Since Last Report, Give Reasons:

10. Power Level To Which Restricted, If Any (Net MWe):

11. Reasons For Restrictions, If Any:

\begin{tabular}{|c|c|c|c|}
\hline & MONTH & YEAR & CUMULAT IVE \\
\hline 12. Report Period Hrs & 744.0 & $8,760.0$ & $12,376.0$ \\
\hline 13. Hours Reactor Critical & 744.0 & $5,828.0$ & $9,116.0$ \\
\hline 14. Rx Reserve Shtdwn Hrs & 0.0 & $1,827.0$ & $2,155.0$ \\
\hline 15. Hrs Generator On-Line & 744.0 & $5,697.0$ & $8,942.3$ \\
\hline 16. Unit Reserve Shtdwn Hrs & 0.0 & 0.0 & 0.0 \\
\hline 17. Gross Therm Ener (MWH) & $2,534,851.0$ & $16,681,968.0$ & $27,299,926.0$ \\
\hline 18. Gross Elec Ener (MWH) & $864,418.0$ & $5,568,828.0$ & $9,156,055.0$ \\
\hline 19. Het Elec Ener (MWH) & $832,833.0$ & $5,263,150.0$ & $8,694,956.0$ \\
\hline 20. Unit Service Factor & 100.0 & 65.0 & 72.3 \\
\hline 21. Unit Avail Factor & 100.0 & 65.0 & 72.3 \\
\hline 22. Unit Cap Factor (MDC Net) & 97.3 & 52.2 & 61.1 \\
\hline 23. Unit Cap Factor (DER Net) & 97.3 & 52.2 & 61.1 \\
\hline 24. Unit Forced Outage Rate & 0.0 & 17.1 & 12.1 \\
\hline 25. Forced Outage Hours & 0.0 & 1172.3 & $1,229.3$ \\
\hline
\end{tabular}

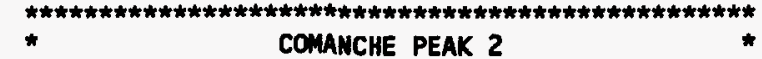

COMANCHE PEAK 2

AVERAGE DAILY POUER LEVEL (Net MHe)

\begin{tabular}{ll} 
DAY & PONER \\
1 & 1113 \\
\hline 2 & 1113 \\
\hline 3 & 1113 \\
\hline 4 & 1113 \\
\hline 5 & 1122 \\
\hline 6 & 1120 \\
\hline 7 & 1119 \\
\hline 8 & 1116 \\
\hline 9 & 1119 \\
\hline 10 & 1119 \\
\hline 11 & 1119 \\
\hline 12 & 1118 \\
\hline 13 & 1119 \\
\hline 14 & 1119 \\
\hline 15 & 1120 \\
\hline
\end{tabular}

\begin{tabular}{ll} 
DAY & PONER \\
16 & 1120 \\
\hline 17 & 1121 \\
\hline 18 & 1120 \\
\hline 19 & 1120 \\
\hline 20 & 1121 \\
\hline 21 & 1121 \\
\hline 22 & 1121 \\
\hline 23 & 1122 \\
\hline 24 & 1121 \\
\hline 25 & 1121 \\
\hline 26 & 1124 \\
\hline 27 & 1121 \\
\hline 28 & 1122 \\
\hline 29 & 1120 \\
\hline 30 & 1122 \\
\hline 31 & 1123 \\
\hline
\end{tabular}

26. Shutdowns Scheduled Over Mext Six Months (Type, Date, Duration):

27. If Currently Shutdown, Estimated startup Date:

Notes: 


\begin{tabular}{llllllll} 
No. Date & Type Hours Reason Method LER Number System Component Cause and Corrective Action To Prevent Recurrence \\
\hline
\end{tabular}

TYPE

F: Forced

S: Scheduled

\section{REASON}

A-Equipment Failure
B-Maintenance or Test

B-Maintenance or Test

C-Refuel ing

D-Regulatory Restriction

E-Operator Training \&

License Examination

F-Administrative

G-Operational Error

H-other

\section{METHOO}

1-Manua

2-Manual Scram

3-Auto Scram

4-Cont inued

5-Reduced Load

9-Other

\section{SYSTEM}

IEEE Standard

805-1984 and/or

NUREG-0161 Exhibit F

\section{COMPONENT}

IEEE Standard

803A-1983 and/or

NUREG-0161 Exhibit 


\section{FACILITY DESCRIPTION}

\section{LOCATION}

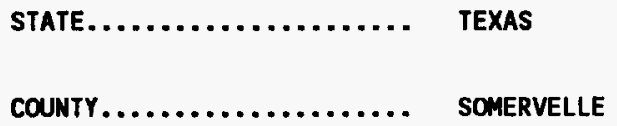

\section{UTILITY \& CONTRACTOR INFORMATION}

UTILITY

LICENSEE. .

CORPORATE ADDRESS

400 N. OLIVE STREET DALLAS, TEXAS 75201

\section{CONTRACTOR}

ARCHITECT/ENGINEER.......... STONE \& WEBSTER

NUC STEAM SYS SUPPLIER...... WESTINGHOUSE

CONSTRUCTOR

BRONN \& ROOT

TURBINE SUPPLIER.

\section{ALLIS-CHALMERS}

\section{REGULATORY INFORMATION}

IE REGION RESPONSIBLE........ 4

IE RESIDENT INSPECTOR.

ANTHONY GCOY, JR.

LICENSING PROJ MANAGER

THOMAS A. BERGMAN

DOCKET NUMBER

50-446

LICENSE \& DATE ISSUANCE.

NPF-089, APRIL 06, 1993 
1. Docket: $50-315$

OPERATING

S T A T U S

2. Reporting Period: DECEMBER 1994

Outage + On-Line Hrs:

744.0

3. Utility Contact: J. D. JANISSE (616) 465-5901 EXT. 1570

4. Licensed Thermal Power (MHt):

5. Nameplate Rating (Gross MWe):

6. Design Electrical Rating (Net MWe):

7. Maximum Dependable Capacity (Gross MUe):

8. Maximum Dependable Capacity (Net MWe):

9. If Changes Occurred Above Since Last Report, Give Reasons:

10. Power Level To Which Restricted, If Any (Net MHe):

11. Reasons for Restrictions, If Any:

\begin{tabular}{|c|c|c|c|}
\hline & MONTH & YEAR & CUMULATIVE \\
\hline 12. Report Period Hrs & 744.0 & $8,760.0$ & $175,320.0$ \\
\hline 13. Hours Reactor Critical & 744.0 & $6,256.5$ & $131,909.9$ \\
\hline 14. RX Reserve Shtdun Hrs & 0.0 & 0.0 & 463.0 \\
\hline 15. Hrs Generator On-Line & 744.0 & $6,215.0$ & $129,822.6$ \\
\hline 16. Unit Reserve Shtdun Hrs & 0.0 & 0.0 & 321.0 \\
\hline 17. Gross Therm Ener (MUH) & $2,414,279.0$ & $18,594,801.0$ & $382,120,584.0$ \\
\hline 18. Gross Elec Ener (MWH) & $790,390.0$ & $5,982,780.0$ & $124,005,440.0$ \\
\hline 19. Net Elec Ener (MWH) & $763,062.0$ & $5,759,517.0$ & $119,281,315.0$ \\
\hline 20. Unit Service Factor & 100.0 & 70.9 & 74.0 \\
\hline 21. Unit Avail Factor & 100.0 & 70.9 & 74.2 \\
\hline 22. Unit Cap Factor (MDC Net) & 102.6 & 65.7 & 67.8 \\
\hline 23. Unit Cap Factor (DER Net) & 100.6 & 64.5 & 66.0 \\
\hline 24. Unit Forced Outage Rate & 0.0 & 0.0 & 5.7 \\
\hline 25. Forced outage Hours & 0.0 & 0.0 & $7,800.6$ \\
\hline
\end{tabular}

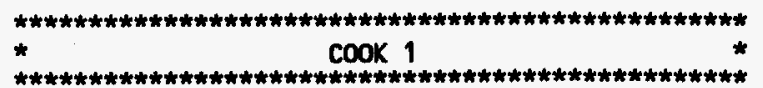

AVERAge dAILY POWER LeVEL (Net MWe)

\begin{tabular}{ll} 
DAY & PONER \\
\hline 1 & 1023 \\
\hline 2 & 1030 \\
\hline 3 & 1022 \\
\hline 4 & 1024 \\
\hline 5 & 1019 \\
\hline 6 & 1024 \\
\hline 7 & 1019 \\
\hline 8 & 1025 \\
\hline 9 & 1023 \\
\hline 10 & 1025 \\
\hline 11 & 1021 \\
\hline 12 & 1024 \\
\hline 13 & 1024 \\
\hline 14 & 1025 \\
\hline 15 & 1024 \\
\hline
\end{tabular}

\begin{tabular}{cc} 
DAY & PONER \\
\hline 16 & 1025 \\
\hline 17 & 1026 \\
\hline 18 & 1031 \\
\hline 19 & 1029 \\
\hline 20 & 1027 \\
\hline 21 & 1028 \\
\hline 22 & 1027 \\
\hline 23 & 1029 \\
\hline 24 & 1027 \\
\hline 25 & 1033 \\
\hline 26 & 1031 \\
\hline 27 & 1029 \\
\hline 28 & 1030 \\
\hline 29 & 1030 \\
\hline 30 & 1017 \\
\hline 31 & 1023 \\
\hline
\end{tabular}

26. Shutdowns Scheduled Over Mext Six Months (Type, Date, Duration):

27. If Currently Shutdown, Estimated Startup Date:

Notes:

CUMULATIVE UNIT CAPACITY FACTORS (MDC \& DER) ARE CALCULATED WITH WEIGHTED AVERAGES. 
TYPE

F: Forced

S: Scheduled

\section{REASON}

A-Equipment Failure

B-Maintenance or Test

C-Refuel ing

D-Regulatory Restriction

E-Operator Training \&

License Examination

F-Administrative

G-Operational Error

H-Other

\begin{tabular}{l} 
METHOD \\
\hline 1-Manual \\
2-Manual Scram \\
3-Auto Scram \\
4-Cont inued \\
5-Reduced Load \\
9-Other
\end{tabular}

SYSTEM

IEEE Standard

$805-1984$ and/or

NUREG-0161 Exhibit F

COMPONEN

IEEE Standard

$803 A-1983$ and/or

NUREG-0161 Exhibit H 


\section{UTILITY \& CONTRACTOR INFORMATION}

\section{FACILITY DESCRIPTION}

\section{LOCATION}

STATE

MICHIGAN

COUNTY

BERRIEN

\section{DIST AND DIRECTION FROM}

MEAREST POPULATION CTR...

TYPE OF REACTOR

11 MI S OF BENTON HARBOR, MI

PUR

DATE INITIAL CRITICALITY...... JANUARY 18, 1975

DATE INITIAL ELECTRICITY...... FEBRUARY 10, 1975

DATE COMmERCial OPERATE....... August 27, 1975

CONDENSER COOLING METHOD...... ONCE THRU

CONDENSER COOLING HATER....... LAKE MICHIGAN

ELECTRIC RELIABILITY

COUNCIL.................. EAST CENTRAL AREA RELIABILITY

COORDINATION AGREEMENT

\section{UTILITY}

LICENSEE.

CORPORATE ADDRESS.

\section{CONTRACTOR}

ARCHI TECT/ENGINEER.

NUC STEAM SYS SUPPLIER...... MESTIMGHOUSE

CONSTRUCTOR

TURBINE SUPPLIER

AMERICAN ELEC. POWER SERVICE CORP.

GENERAL ELECTRIC

\section{REGULATORY INFORMATION}

IE REGION RESPONSIBLE.

3

IE RESIDENT INSPECTOR

JAMES ISOM

LICENSING PROJ MANAGER.

JOHN B. HICKMAN

DOCKET NUMBER .

$50-315$

LICENSE \& DATE ISSUANCE.

IMDIANA MICHIGAN ELECTRIC CO.

1 RIVERSIDE PLAZA

COLUMBUS, OHIO 43215

MERICAN ELEC. PONER SERVICE CORP. 
1. Docket: $50-316$

OPERATING STATUS

2. Reporting Period: DECEMBER 1994

Outage + On-Line Hrs: $\quad \mathbf{7 4 4 . 0}$

3. Utility Contact: J. D. JANISSE (616) 465-5901 EXT. 1570

4. Licensed Thermal Power (MWt):

\begin{tabular}{c}
3411 \\
\hline 1133 \\
\hline 1090 \\
\hline 1100 \\
\hline 1060
\end{tabular}

5. Nameplate Rating (Gross MWe):

6. Design Elactrical Rating (Net MWe):

7. Maximum Dependable Capacity (Gross MHe):

8. Maximum Dependable Capacity (Net MHe):

9. If Changes Occurred Above Since Last Report, Give Reasons:

10. Power Level To which Restricted, If Any (Net MHe):

11. Reasons for Restrictions, If Any:

\begin{tabular}{|c|c|c|c|}
\hline & MONTH & YEAR & CUMULATIVE \\
\hline 12. Report Periad Hrs & 744.0 & $8,760.0$ & 149.016 .0 \\
\hline 13. Hours Reactor Critical & 492.4 & $5,167.5$ & $100,016.5$ \\
\hline 14. Rx Reserve Shtdwn Hrs & 0.0 & 0.0 & 0.0 \\
\hline 15. Hrs Generator On-Line & 438.1 & 4.759 .2 & $96,535.1$ \\
\hline 16. Unit Reserve Shtdwn Hrs & 0.0 & 0.0 & 0.0 \\
\hline 17. Gross Therm Ener (MUH) & $1,006,215.0$ & $11,984,087.0$ & $294,098,183.0$ \\
\hline 18. Gross Elec Ener (MHH) & $312,580.0$ & $3,699,090.0$ & $95,306,990.0$ \\
\hline 19. Het Elec Ener (MWH) & $297,086.0$ & $3,531,453.0$ & $91,757,435.0$ \\
\hline 20. Unit Service Factor & 58.9 & 54.3 & 64.8 \\
\hline 21. Unit Avail Factor & 58.9 & 54.3 & 64.8 \\
\hline 22. Unit Cap Factor (MDC Net) & 37.7 & 38.0 & 58.9 \\
\hline 23. Unit Cap Factor (DER Net) & 36.6 & 37.0 & 57.4 \\
\hline 24. Unit Forced Outage Rate & 10.4 & 26.1 & 15.7 \\
\hline 25. Forced Outage Hours & 50.7 & 1684.0 & $18,044.3$ \\
\hline
\end{tabular}

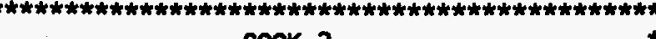 \\ $\operatorname{coOK} 2$}

AVERAGE DAILY POWER LEVEL (Net MWe)

\begin{tabular}{|c|c|c|c|}
\hline DAY & POWER & DAY & POUER \\
\hline 1 & 0 & 16 & 426 \\
\hline 2 & 0 & 17 & 426 \\
\hline 3 & 0 & 18 & 434 \\
\hline 4 & 0 & 19 & 536 \\
\hline 5 & 0 & 20 & 558 \\
\hline 6 & 0 & 21 & 566 \\
\hline 7 & 0 & 22 & 683 \\
\hline 8 & 0 & 23 & 699 \\
\hline 9 & 0 & 24 & 706 \\
\hline 10 & 0 & 25 & 705 \\
\hline 11 & 9 & 26 & 803 \\
\hline 12 & 0 & 27 & 972 \\
\hline 13 & 28 & 28 & 1058 \\
\hline 14 & 237 & 29 & 1084 \\
\hline \multirow[t]{2}{*}{15} & 278 & 30 & 1087 \\
\hline & & 31 & 1083 \\
\hline
\end{tabular}

26. Shutdowns Scheduled Over Next Six Months (Type, Date, Duration):

27. If Currently Shutdoun, Estimated Startup Date:

Notes:

CUMULATIVE UNIT CAPACITY FACTORS (MDC \& DER) ARE CALCULATED WITH WEIGHTED AVERAGES. 


\begin{tabular}{|c|c|c|c|c|c|c|c|c|c|}
\hline No. & Date & Type & Hours & Reason & Method & LER Number & System & Component & Cause and Corrective Action To Prevent Recurrence \\
\hline 239 & $09 / 06 / 94$ & $\mathbf{s}$ & 255.2 & C & 4 & & RC & FUELXX & REFUELING/MAINTENANCE OUTAGE CONTINUED. \\
\hline 240 & $12 / 11 / 94$ & $\mathbf{F}$ & 50.7 & H & 3 & 94008 & IB & LS & $\begin{array}{l}\text { DURING PONER ESCALATION, AN MSR HIGH LEVEL TRIP } \\
\text { SIGNAL WAS GENERATED FOLLOWED BY A REACTOR TRIP. } \\
\text { THE CAUSE OF THE TRIP SIGMAL COULD NOT BE } \\
\text { CONCLUSIVELY DETERMINED. }\end{array}$ \\
\hline
\end{tabular}

\begin{tabular}{|c|c|}
\hline TYPE & REASON \\
\hline $\begin{array}{l}\text { F: Forced } \\
\text { S: Scheduled }\end{array}$ & $\begin{array}{l}\text { A-Equipment Fai lure } \\
\text { B-Maintenance or Test } \\
\text { C-Refuel ing } \\
\text { D-Regulatory Restriction } \\
\text { E-Operator Training \& } \\
\text { License Examination } \\
\text { F-Administrat ive } \\
\text { G-Operat ional Error } \\
\text { H-Other }\end{array}$ \\
\hline
\end{tabular}

\begin{tabular}{l} 
METHOD \\
\hline 1-Manual \\
2-Manual Scram \\
3-Auto Scram \\
4-Cont inued \\
5-Reduced Load \\
9-0ther
\end{tabular}

SYSTEN

IEEE Standard

805-1984 and/or

NUREG-0161 Exhibit F
COMPONENT

IEEE Standard

803A-1983 and/or

NUREG-0161 Exhibit H 


\section{FACILITY DESCRIPTION}

\section{LOCATIOH}

$$
\begin{aligned}
& \text { STATE } \ldots \ldots \ldots \ldots \ldots \ldots \ldots \ldots \text { MICHIGAN } \\
& \text { COUNTY................. BERRIEN }
\end{aligned}
$$

\section{DIST AND DIRECTION FROM}

NEAREST POPULATION CTR..... 11 MI S OF BENTON HARBOR, MI

TYPE OF REACTOR

DATE INITIAL CRITICALITY....... MARCH 10, 1978

DATE INITIAL ELECTRICITY...... MARCH 22, 1970

DATE COMMERCIAL OPERATE....... JULY 01, 1978

CONDENSER COOLING METHOO...... ONCE THRU

CONDENSER COOLING MATER....... LAKE MICHIGAN

ELECTRIC RELIABILITY

COUNCIL.................. EAST CENTRAL AREA RELIABILITY

\section{UTILITY \& CONTRACTOR INFORMATION}

UTILITY

LICENSEE...

CORPORATE ADDRESS.

IMDIAMA MICHIGAN ELECTRIC CO.

1 RIVERSIDE PLAZA

COLUMBUS, OHIO 43215

\section{CONTRACTOR}

ARCHITECT/ENGINEER.......... AMERICAN ELEC. PONER SERVICE CORP.

NUC STEAM SYS SUPPLIER ..... MESTIMGHOUSE

CONSTRUCTOR............... AMERICAN Elec. PONER service CORP.

TURBINE SUPPLIER.......... BROWN BOVERI

\section{REGULATORY INFORMATION}

IE REGION RESPONSIBLE......... 3

IE RESIDENT INSPECTOR.

JAMES ISOW

LICENSING PROJ MANAGER.

JOHN B. HICKMAI

DOCKET NUMBER

$50-316$

LICENSE \& DATE ISSUANCE.

DPR 074, DECEMBER 23, 1977 
1. Docket: $50-298$

OPERATING STATUS

2. Reporting Period: DECEMBER 1994

Outage + On-Line Hrs: $\mathbf{7 4 4 . 0}$

3. Utility Contact: E. A. KERNES KRAUSE (402) 825-5829

4. Licensed Thermal Power (MUt):

5. Nameplate Rating (Gross MHe):

6. Design Electrical Rating (Net MHe):

7. Maximum Dependable Capacity (Gross MWe):

8. Maximum Dependable Capacity (Net MWe):

9. If Changes Occurred Above Since Last Report, Give Reasons:

10. Power Level To which Restricted, If Any (Net MHe):

11. Reasons for Restrictions, If Any:

\begin{tabular}{|c|c|c|c|}
\hline & MONTH & YEAR & CUMULATIVE \\
\hline 12. Report Period Hrs & 744.0 & $8,760.0$ & 179.737 .0 \\
\hline 13. Hours Reactor Critical & 0.0 & $3,076.4$ & $133,190.2$ \\
\hline 14. Rx Reserve Shtdwn Hrs & 0.0 & 0.0 & 0.0 \\
\hline 15. Hrs Generator On-Line & 0.0 & $3,033.0$ & $131,279.3$ \\
\hline 16. Unit Reserve Shtdwn Hrs & 0.0 & 0.0 & 0.0 \\
\hline 17. Gross Therm Ener (MWH) & 0.0 & $6,953,352.0$ & $271,311,244.0$ \\
\hline 18. Gross Elec Ener (MWH) & 0.0 & $2,296,438.0$ & $88,118,336.0$ \\
\hline 19. Net Elec Ener (MWH) & 0.0 & $2,227,260.0$ & $85,098,438.0$ \\
\hline 20. Unit Service Factor & 0.0 & 34.6 & 73.0 \\
\hline 21. Unit Avail Factor & 0.0 & 34.6 & 73.0 \\
\hline 22. Unit Cap Factor (MDC Net) & 0.0 & 33.3 & 62.0 \\
\hline 23. Unit Cap Factor (DER Net) & 0.0 & 32.7 & 60.9 \\
\hline 24. Unit Forced Outage Rate & 100.0 & 65.4 & 8.0 \\
\hline 25. Forced Outage Hours & 744.0 & 5727.0 & $11,422.0$ \\
\hline
\end{tabular}

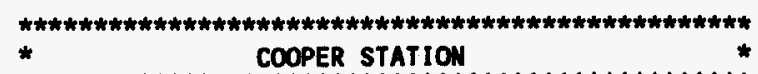

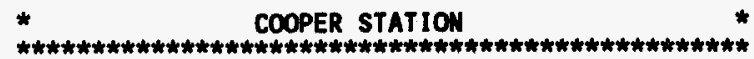

AVERAGE DAILY PONER LEVEL (Net MWe)

\begin{tabular}{lr} 
DAY & POWER \\
\hline 2 & 0 \\
\hline 3 & 0 \\
\hline 4 & 0 \\
\hline 5 & 0 \\
\hline 6 & 0 \\
\hline 7 & 0 \\
\hline 8 & 0 \\
\hline 9 & 0 \\
\hline 10 & 0 \\
\hline 11 & 0 \\
\hline 12 & 0 \\
\hline 13 & 0 \\
\hline 14 & 0 \\
\hline 15 & 0 \\
\hline
\end{tabular}

\begin{tabular}{cc} 
DAY & PONER \\
\hline 16 & 0 \\
\hline 17 & 0 \\
\hline 18 & 0 \\
\hline 19 & 0 \\
\hline 20 & 0 \\
\hline 21 & 0 \\
\hline 22 & 0 \\
\hline 23 & 0 \\
\hline 24 & 0 \\
\hline 25 & 0 \\
\hline 26 & 0 \\
\hline 27 & 0 \\
\hline 28 & 0 \\
\hline 29 & 0 \\
\hline 30 & 0 \\
\hline 31 & 0 \\
\hline
\end{tabular}

26. Shutdowns Scheduled Over Mext Six Months (Type, Date, Duration):

27. If Currently Shutdown, Estimated Startup Date: 01/29/95

Notes: 
A N D

POWER

REDUCTIONS

\begin{tabular}{cccccccccc} 
No. & Date & Type & Hours & Reason & Method & LER Number & System & Component & Cause and Corrective Action To Prevent Recurrence \\
\hline $94-5$ & $05 / 25 / 94$ & F & 744.0 & $H$ & 4 & 94009 & EK & 27 & EDG 1 AND EDG 2 DECLARED INOPERABLE DUE TO \\
INSUFFICIENT UV RELAY TESTING.
\end{tabular}

$\frac{\text { TYPE }}{\text { F: Forced }}$

\section{REASON}

A-Equipment Failure

B-Maintenance or Test

C-Refuel ing

D-Regulatory Restriction

E-operator Training \&

License Examination

F-Administrative

G-Operational Error

H-other

\begin{tabular}{l} 
METHOD \\
\hline 1-Manual \\
2-Manual Scram \\
3-Auto Scram \\
4-Cont inued \\
5-Reduced Load \\
9-0ther
\end{tabular}

SYSTEM

IEEE Standard

805-1984 and/or

NUREG-0161 Exhibit $F$

IEEE Standard

803A-1983 and/or

NUREG-0161 Exhibit H

5-Cont inued 


\section{FACILITY DESCRIPTION}

\section{LOCATION}

$$
\begin{aligned}
& \text { STATE................. MEBRASKA } \\
& \text { COUNTY............... MEMAHA }
\end{aligned}
$$

\section{DIST AND DIRECTION FROM}

NEAREST POPULATION CTR..... 23 MI S OF NEBRASKA CITY, NE

TYPE OF REACTOR

DATE INITIAL CRITICALITY....... FEBRUARY 21, 1974

DATE INITIAL ELECTRICITY...... MAY 10, 1974

DATE COMMERCIAL OPERATE....... JULY 01, 1974

CONDENSER COOLING METHOD..... ONCE THRU

CONDENSER COOLING WATER....... MISSOURI RIVER

ELECTRIC RELIABILITY

COUNCIL................... MID-CONTINENT AREA PONER POOL

\section{UTILITY \& CONTRACTOR INFORMATION}

UTILITY

LICENSEE............... MEBRASKA PUBLIC PONER DISTRICT

CORPORATE ADDRESS........... P.0. BOX 499

\section{P.O. BOX 499 . 68601}

\author{
CONTRACTOR \\ ARCHITECT/ENGINEER......... : BURNS \& ROE \\ NUC STEAM SYS SUPPLIER...... GEMERAL ELECTRIC \\ CONSTRUCTOR.............. BURNS \& ROE \\ TURBIME SUPPLIER........... VESTINGHOUSE
}

REGULATORY INFORMATION

IE REGION RESPONSIBLE........ 4

IE RESIDENT INSPECTOR.......... RONALD KOPRIVA

LICENSING PROJ MAMAGER......... JAMES RANDALL HALL

DOCKET NUMBER............... 50-298

LICENSE \& DATE ISSUANCE........ DPR 046, JANUARY 18, 1974 


\section{Docket: $50-302$}

OPERATING STATUS

2. Reporting Period: DECEMBER 1994

Outage + On-Line Hrs: 744.0

3. Utility Contact: T. V. DAO (904) 795-6486

4. Licensed Thermal Power (MWt):

5. Nameplate Rating (Gross MHe):

6. Design Electrical Rating (Net MWe):

7. Maximum Dependable Capacity (Gross MWe):

8. Maximum Dependable Capacity (Net MWe):

9. If Changes Occurred Above Since Last Report, Give Reasons:

10. Power Level To Which Restricted, If Any (Net MWe):

11. Reasons For Restrictions, If Any:

\begin{tabular}{|c|c|c|c|}
\hline & MONTH & YEAR & CUMULATIVE \\
\hline 12. Report Period Hrs & 744.0 & $8,760.0$ & $156,072.0$ \\
\hline 13. Hours Reactor Critical & 744.0 & $7,382.0$ & $105,348.0$ \\
\hline 14. Rx Reserve Shtdwn Hrs & 0.0 & 0.0 & $1,280.6$ \\
\hline 15. Hrs Generator On-Line & 702.7 & $7,293.7$ & $103,486.0$ \\
\hline 16. Unit Reserve Shtdwn Hrs & 0.0 & 0.0 & 0.0 \\
\hline 17. Gross Therm Ener (MUH) & $1,777,658.0$ & $18,205,765.0$ & $239,347,168.0$ \\
\hline 18. Gross Elec Ener (MUH) & $609,946.0$ & $6,236,617.0$ & $81,757,519.0$ \\
\hline 19. Net Elec Ener (MWH) & $569,608.0$ & $5,939,857.0$ & $77.750,068.0$ \\
\hline 20. Unit Service Factor & 94.4 & 83.3 & 66.3 \\
\hline 21. Unit Avail Factor & 94.4 & 83.3 & 66.3 \\
\hline 22. Unit Cap Factor (MDC Net) & 93.6 & 82.9 & 61.7 \\
\hline 23. Unit Cap Factor (DER Net) & 92.8 & 82.2 & 60.4 \\
\hline 24. Unit forced Outage Rate & 0.0 & 0.0 & 16.3 \\
\hline 25. Forced Outage Hours & 0.0 & 0.0 & $20,142.9$ \\
\hline
\end{tabular}

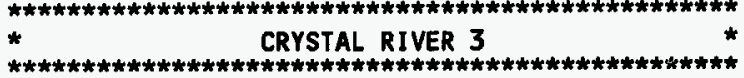

AVERAGE DAILY POWER LEVEL (Net MHe)

\begin{tabular}{|c|c|c|c|}
\hline DAY & POWER & DAY & PONER \\
\hline 1 & 842 & 16 & 839 \\
\hline 2 & 779 & 17 & 844 \\
\hline 3 & 12 & 18 & 844 \\
\hline 4 & 45 & 19 & 844 \\
\hline 5 & 696 & 20 & 843 \\
\hline 6 & 841 & 21 & 844 \\
\hline 7 & 843 & 22 & 844 \\
\hline 8 & 842 & 23 & 844 \\
\hline 9 & 842 & 24 & 844 \\
\hline 10 & 843 & 25 & 844 \\
\hline 11 & 842 & 26 & 844 \\
\hline 12 & 842 & 27 & 844 \\
\hline 13 & 843 & 28 & 844 \\
\hline 14 & 844 & 29 & 844 \\
\hline \multirow[t]{2}{*}{15} & 844 & 30 & 844 \\
\hline & & 31 & 844 \\
\hline
\end{tabular}

26. Shutdowns Scheduled Over Next six Months (Type, Date, Duration):

27. If Currently Shutdown, Estimated Startup Date:

Notes:

YEAR-TO-DATE AND CUMULATIVE UNIT CAPACITY FACTORS (MDC NET) ARE CALCULATED WITH WEIGHTED AVERAGES. 
$x \times x \times x x$

GENERATOR WAS TAKEN OFF LIME TO REPLACE ISOPHASE BUS DUCT GROUND STRAPS.

TYPE

F: Forced

S: Scheduled
REASON

A-Equipment Failure B-Maintenance or Test C-Refuel ing

D-Regulatory Restriction

E-Operator Training \&

License Examination

F-Administrative

G-Operational Error

H-other

\section{SYSTEM}

IEEE Standard

805-1984 and/or

NUREG-0161 Exhibit $F$

\section{COMPONENT}

1-Manual

3-Auto Scram

4-Cont inued

5 -Reduced Load

9-other

\section{IEEE Standard \\ 803A-1983 and/or}

NUREG-0161 Exhibit H 


\section{FACILITY DESCRIPTION}

LOCATION

STATE.

COUNTY

DIST AND DIRECTION FROM MEAREST POPULATION CTR.

TYPE OF REACTOR.

DATE INITIAL CRITICALITY...... JANUARY 14, 1977

DATE INITIAL ELECTRICITY...... J JANUARY 30, 1977

DATE COMMERCIAL OPERATE....... MARCH 13,1977

CONDENSER COOLING METHOD...... ONCE THRU

CONDENSER COOLING MATER....... GULF OF MEXICO

ELECTRIC RELIABILITY

COUNCIL.................. SOUTHEASTERH ELECTRIC RELIABILITY COUNCIL

\section{UTILITY \& CONTRACTOR INFORMATION}

UTILITY

LICENSEE.

FLORIDA PONER CORPORATION

CORPORATE ADDRESS.

\section{P.O. BOX 14042}

ST. PETERSBURG, FLORIDA 33733

CONTRACTOR

ARCHI TECT/ENGINEER

GILBERT ASSOCIATES

NUC STEAM SYS SUPPLIER

BABCOCK \& HILCOX

CONSTRUCTOR.

J. A. JONES CONSTRUCTION

TURBINE SUPPLIER

WESTINGHOUSE

\section{REGULATORY INFORMATION}

IE REGION RESPONSIBLE......... 2

IE RESIDENT INSPECTOR.......... ROSS BUTCHER

LICENSING PROJ MANAGER........ LAKSHMINARASIMH RAGHAVAN

DOCKET MUMBER.............. $50-302$

LICENSE \& DATE ISSUANCE....... DPR 072, JANUARY 28, 1977 
1. Docket: $50-346$

OPERATING

S TA T U S

2. Reporting Period: DECEMBER 1994

Outage + On-Line Hrs:

744.0

3. Utility Contact: GERRY WOLF (419) 321-8114

4. Licensed Thermal Power (MUt):

5. Naneplate Rating (Gross MHe):

6. Design Electrical Rating (Net MHe):

7. Maximum Dependable Capacity (Gross MWe):

8. Maximum Dependable Capacity (Net MWe):

\begin{tabular}{c}
\hline 2772 \\
\hline 925 \\
\hline 906 \\
\hline 913 \\
\hline 868
\end{tabular}

9. If Changes Occurred Above Since Last Report, Give Reasons:

10. Power Level To thich Restricted, If Any (Net MWe):

11. Reasons for Restrictions, If Any:

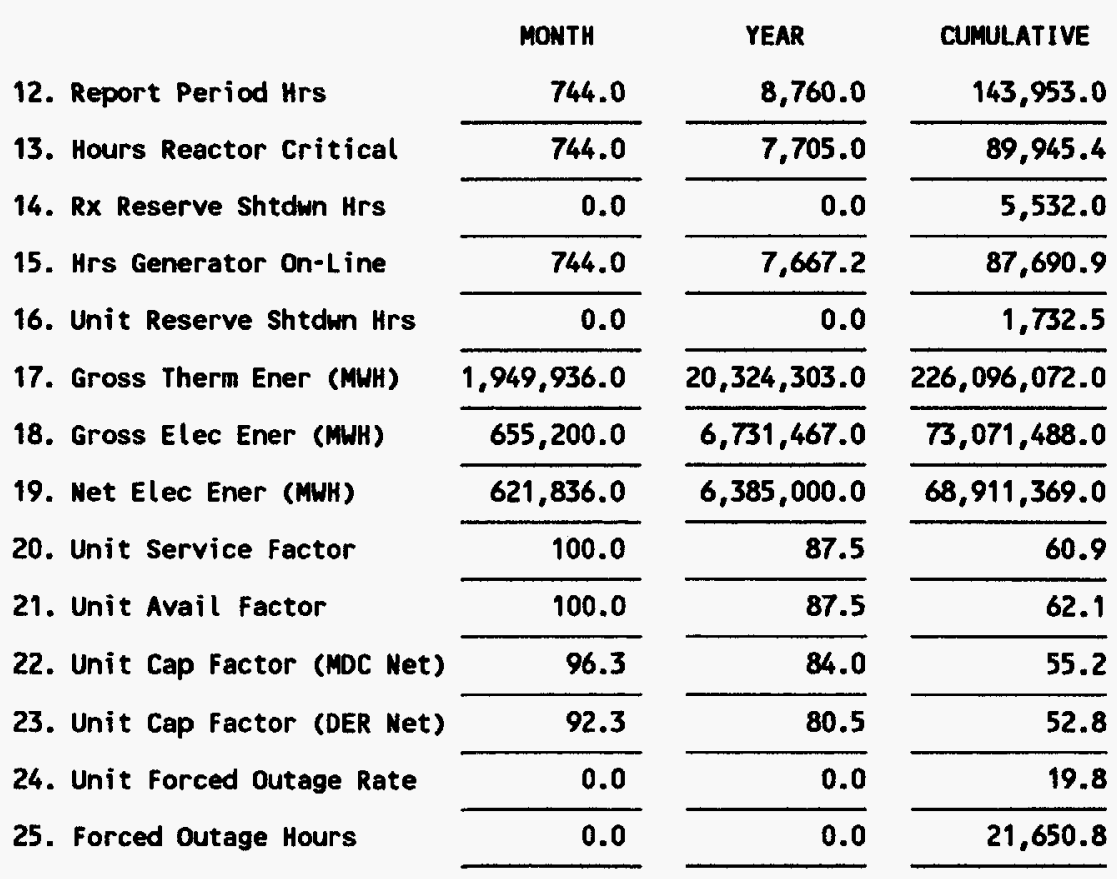

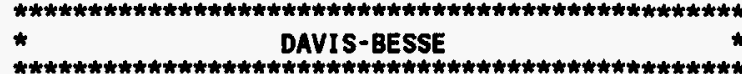

AVERAge DAILY PONER LEVEL (Net MUe)

\begin{tabular}{lc} 
DAY & PONER \\
\hline 1 & 742 \\
\hline 2 & 521 \\
\hline 3 & 518 \\
\hline 4 & 520 \\
\hline 5 & 689 \\
\hline 6 & 881 \\
\hline 7 & 881 \\
\hline 8 & 884 \\
\hline 9 & 885 \\
\hline 10 & 885 \\
\hline 11 & 885 \\
\hline 12 & 885 \\
\hline 13 & 883 \\
\hline 14 & 880 \\
\hline 15 & 886 \\
\hline
\end{tabular}

\begin{tabular}{cc} 
DAY & POUER \\
\hline 16 & 883 \\
\hline 17 & 883 \\
\hline 18 & 884 \\
\hline 19 & 885 \\
\hline 20 & 884 \\
\hline 21 & 883 \\
\hline 22 & 884 \\
\hline 23 & 885 \\
\hline 24 & 884 \\
\hline 25 & 851 \\
\hline 26 & 860 \\
\hline 27 & 885 \\
\hline 28 & 882 \\
\hline 29 & 884 \\
\hline 30 & 884 \\
\hline 31 & 884 \\
\hline
\end{tabular}

26. Shutdowns Scheduled Over Mext six Months (Type, Date, Duration):

27. If Currently Shutdown, Estimated Startup Date:

Notes:

LICENSEE REVISED CUMULATIVE GROSS THERMAL EMERGY VALUE TO ACCOUNT FOR PAST CALCULATIONAL ERRORS. 


$\begin{array}{llllll}12 & 12 / 01 / 94 & F & 0.0 & B & 5\end{array}$

POWER REDUCED TO CORRECT THE HIGH VIBRATION ON MAIN FEED PUMP TURBINE ONE.

\begin{tabular}{|c|c|}
\hline TYPE & REASON \\
\hline $\begin{array}{l}\text { F: Forced } \\
\text { s: Scheduled }\end{array}$ & $\begin{array}{l}\text { A-Equipment Failure } \\
\text { B-Maintenance or Test } \\
\text { C-Refuel ing } \\
\text { D-Regulatory Restriction } \\
\text { E-Operator Training \& } \\
\text { License Examination } \\
\text { F-Administrative } \\
\text { G-Operational Error } \\
\text { H-Other }\end{array}$ \\
\hline
\end{tabular}

\begin{tabular}{l} 
METHOD \\
\hline 1-Manual \\
2-Manual Scram \\
3-Auto Scram \\
4-Cont inued \\
5-Reduced Load \\
9-Other
\end{tabular}

\section{SYSTEN}

IEEE Standard

$805-1984$ and/or

NUREG-0161 Exhibit $F$

\section{COMPONENT}

IEEE Standard

$803 \mathrm{~A}-1983$ and/or

NUREG-0161 Exhibit H 


\section{FACILITY DESCRIPTION}

LOCATION

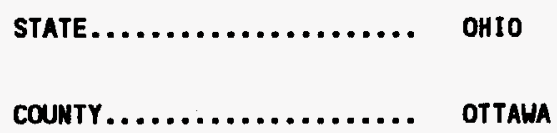

\section{DIST AND DIRECTION FROM}

MEAREST POPULATION CTR.

TYPE OF REACTOR

21 MI ESE OF TOLEDO, OH

PUR

DATE INITIAL CRITICALITY...... AUGUST 12, 1977

DATE INITIAL ELECTRICITY...... AUGUST 28, 1977

DATE COMMERCIAL OPERATE........ JULY 31, 1978

CONDENSER COOLING METHOD...... COOLING TOMER

CONDENSER COOLING WATER....... LAKE ERIE

ELECTRIC RELIABILITY

COUNCIL.................. EAST CENTRAL AREA RELIABILITY COORD INATION AGREEMENT

\section{UTILITY \& CONTRACTOR INFORMATION}

UTILITY

LICENSEE.

TOLEDO EDISON CO.

CORPORATE ADDRESS.

300 MADISON AVENUE TOLEDO, OHIO 43652

\section{CONTRACTOR}

ARCHITECT/ENGINEER......... BECHTEL

NUC STEAM SYS SUPPLIER...... BABCOCK \& WILCOX

CONSTRUCTOR............... BECHTEL

TURBINE SUPPLIER........... GENERAL ELECTRIC

\section{REGULATORY INFORMATION}

IE REGION RESPONSIBLE......... 3

IE RESIDENT INSPECTOR.......... STANLEY STASEK

LICENSING PROJ MANAGER......... LINDA GUNDRUM

DOCKET NUMBER............. 50-346

LICENSE \& DATE ISSUANCE......... NPF 003, APRIL 22, 1977 
1. Docket: $50-275$

OPERATING STATUS

2. Reporting Period: DECEMBER 1994

Outage + On-Line Hrs:

744.0

3. Utility Contact: T. EUBANK (805) 545-4867

4. Licensed Thermal Power (MUt):

5. Nameplate Rating (Gross MHe):

6. Design Electrical Rating (Net MWe):

7. Maximum Dependable Capacity (Gross MWe):

8. Maximum Dependable Capacity (Net MWe):

\begin{tabular}{c}
\hline 3338 \\
\hline 1137 \\
\hline 1086 \\
\hline 1124 \\
\hline 1073
\end{tabular}

9. If Changes Occurred Above since Last Report, Give Reasons:

10. Power Level To Which Restricted, If Any (Net MWe):

11. Reasons for Restrictions, If Any:

12. Report Period Hrs
13. Hours Reactor Critical
14. Rx Reserve Shtdun Hrs
15. Hrs Generator On-Line
16. Unit Reserve Shtdwn Hrs
17. Gross Therm Ener (MHH)
18. Gross Elec Ener (MUH)
19. Net Elec Ener (MUH)
20. Unit Service Factor
21. Unit Avail Factor
22. Unit Cap Factor (MDC Net)
23. Unit Cap Factor (OER Net)
24. Unit Forced Outage Rate
25. Forced Outage Hours

\section{YEAR CUMULATIVE}

\begin{tabular}{|c|c|c|c|c|}
\hline \multirow{2}{*}{744.0} & \multirow{2}{*}{$8,760.0$} & \multirow{2}{*}{$84,622.3$} & 10 & 1085 \\
\hline & & & 11 & 1082 \\
\hline 654.1 & $7,041.0$ & $71,284.3$ & 12 & 1081 \\
\hline 0.0 & 0.0 & 0.0 & 13 & 1081 \\
\hline 645.1 & $6,992.4$ & $70,335.4$ & 14 & -19 \\
\hline 0.0 & 0.0 & 0.0 & $\overline{15}$ & -40 \\
\hline .212 .0 & $342,233.0$ & $181,742.0$ & & \\
\hline
\end{tabular}

DIABLO CANYON 1

AVERAGe DAILY POWER LEVEL (Net MHe)

\begin{tabular}{ll} 
DAY & PONER \\
\hline 1 & 1081 \\
\hline 2 & 1085 \\
\hline 3 & 1082 \\
\hline 4 & 1086 \\
\hline 5 & 1081 \\
\hline 6 & 1082 \\
\hline 7 & 1085 \\
\hline 8 & 1077 \\
\hline 9 & 1085 \\
\hline 10 & 1085 \\
\hline 11 & 1082 \\
\hline 12 & 1081 \\
\hline 13 & 1081 \\
\hline 14 & -19 \\
\hline 15 & -40 \\
\hline
\end{tabular}

\begin{tabular}{cc} 
DAY & PONER \\
\hline 16 & -40 \\
\hline 17 & -49 \\
\hline 18 & 262 \\
\hline 19 & 571 \\
\hline 20 & 1009 \\
\hline 21 & 1080 \\
\hline 22 & 1076 \\
\hline 23 & 1077 \\
\hline 24 & 1081 \\
\hline 25 & 1073 \\
\hline 26 & 1082 \\
\hline 27 & 1077 \\
\hline 28 & 1077 \\
\hline 29 & 1077 \\
\hline 30 & 1081 \\
\hline 31 & 1078 \\
\hline
\end{tabular}

26. Shutdowns Scheduled Over Mext Six Months (Type, Date, Duration):

27. If Currently Shutdown, Estimated Startup Date:

Notes:

LICENSEE REVISED CUMULATIVE GROSS THERMAL EMERGY TO IMCLUDE A MAY 1985 REPORT REVISION. 


No. Date TYpe

\begin{tabular}{|c|c|c|c|c|}
\hline TYPE & REASON & METHOD & SYSTEM & COMPONENT \\
\hline $\begin{array}{l}\text { F: Forced } \\
\text { S: Scheduled }\end{array}$ & $\begin{array}{l}\text { A-Equipment Failure } \\
\text { B-Maintenance or Test } \\
\text { C-Refuel ing } \\
\text { D-Regulatory Restriction } \\
\text { E-Operator Training \& } \\
\text { License Examination } \\
\text { F-Administrative } \\
\text { G-Operational Error } \\
\text { H-Other }\end{array}$ & $\begin{array}{l}\text { 1-Manual } \\
\text { 2-Manual Scram } \\
\text { 3-Auto Scram } \\
\text { 4-Cont inued } \\
\text { 5-Reduced Load } \\
\text { 9-0ther }\end{array}$ & $\begin{array}{l}\text { IEEE Standard } \\
805-1984 \text { and/or } \\
\text { MUREG-0161 Exhibit F }\end{array}$ & $\begin{array}{l}\text { IEEE Standard } \\
803 \mathrm{~A}-1983 \text { and/or } \\
\text { NUREG-0161 Exhibit H }\end{array}$ \\
\hline
\end{tabular}




\section{FACILITY DESCRIPTION}

LOCATION

$$
\text { STATE. }
$$

COUNTY.

DIST AND DIRECTION FROM

MEAREST POPULATION CTR

TYPE OF REACTOR

DATE INITIAL CRITICALITY.....

DATE INITIAL ELECTRICITY......

DATE COMMERCIAL OPERATE....... MAY 07, 1985

CONDENSER COOLING METHOD...... ONCE THRU

CONDENSER COOLIMG WATER....... PACIFIC OCEAN

ELECTRIC RELIABILITY

COUNCIL................... WESTERN SYSTEMS COORDINATION \\ UTILITY \& CONTRACTOR INFORMATION}

\section{UTILITY}

LICENSEE.

CORPORATE ADDRESS

PACIFIC GAS \& ELECTRIC CO.

\section{BEALE STREET}

SAN FRANCISCO, CALIFORNIA 94106

\section{CONTRACTOR}

ARCHITECT/ENGINEER

PACIFIC GAS \& ELECTRIC

NUC STEAM SYS SUPPLIER...... MESTINGHOUSE

CONSTRUCTOR.

PACIFIC GAS \& ELECTRIC

TURBINE SUPPLIER.......... WESTINGHOUSE

\section{REGULATORY INFORMATION}

IE REGION RESPONSIBLE........ 4

IE RESIDENT INSPECTOR......... MICHAEL TSCHILTZ

LICENSING PROJ MANAGER......... SHERI R. PETERSON

DOCKET NUMBER............ 50-275

LICENSE \& DATE ISSUANCE........ DPR 080, NOVEMBER 11, 1984 
1. Docket: $50-323$

OPERATING STATUS

2. Reporting Period: DECEMBER 1994

Outage + On-Line Hrs: 744.0

3. Utility Contact: T. EUBANK (805) 545-4867

4. Licensed Thermal Power (MUt):

5. Nameplate Rating (Gross MUe):

6. Design Electrical Rating (Net MWe):

7. Maximum Dependable Capacity (Gross MMe):

8. Maximum Dependable Capacity (Net MWe):

9. If Changes Occurred Above Since Last Report, Give Reasons:

10. Power Level To Which Restricted, If Any (Net MHe):

11. Reasons For Restrictions, If Any:

\begin{tabular}{|c|c|c|c|}
\hline & MONTH & YEAR & CUMULATIVE \\
\hline 12. Report Period Hrs & 744.0 & $8,760.0$ & $77,181.0$ \\
\hline 3. Hours Reactor Critical & 609.8 & $7,560.2$ & $65,780.1$ \\
\hline 4. Rx Reserve Shtdin Hrs & 0.0 & 0.0 & 0.0 \\
\hline 15. Hrs Generator On-Line & 552.9 & $7,441.1$ & $64,772.6$ \\
\hline 6. Unit Reserve Shtdwn Hrs & 0.0 & 0.0 & 0.0 \\
\hline 17. Gross Therm Ener (MUH) & $1,719,242.0$ & $24,810,055.0$ & $212,642,282.0$ \\
\hline 18. Gross Elec Ener (MUH) & $571,900.0$ & $8,289,904.0$ & $70,801,403.0$ \\
\hline 19. Net Elec Ener (MUH) & $537,162.0$ & $7,896,101.0$ & $67,317,865.0$ \\
\hline 20. Unit Service Factor & 74.3 & 84.9 & 83.9 \\
\hline 21. Unit Avail Factor & 74.3 & 84.9 & 83.9 \\
\hline 22. Unit Cap Factor (MDC Net) & 66.4 & 82.9 & 80.4 \\
\hline 23. Unit Cap Factor (DER Net) & 64.5 & 80.6 & 77.9 \\
\hline 24. Unit Forced Outage Rate & 25.7 & 6.2 & 4.2 \\
\hline 5. Forced outage Hours & 191.1 & 491.1 & $2,816.6$ \\
\hline
\end{tabular}

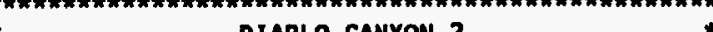 \\ * diablo CANYON 2}

OIABLO CANYON 2

AVERAGE DAILY POWER LEVEL (Net MHe)

\begin{tabular}{ll} 
DAY & POWER \\
\hline 1 & 1096 \\
\hline 2 & 1096 \\
\hline 3 & 1096 \\
\hline 4 & 1101 \\
\hline 5 & 1096 \\
\hline 6 & 1096 \\
\hline 7 & 1101 \\
\hline 8 & 1096 \\
\hline 9 & 1101 \\
\hline 10 & 1097 \\
\hline 11 & 1092 \\
\hline 12 & 1101 \\
\hline 13 & 1197 \\
\hline 14 & -16 \\
\hline 15 & -39 \\
\hline
\end{tabular}

\begin{tabular}{lc} 
DAY & POWER \\
\hline 16 & 107 \\
\hline 17 & 617 \\
\hline 18 & 1062 \\
\hline 19 & 421 \\
\hline 20 & -34 \\
\hline 21 & -38 \\
\hline 22 & -38 \\
\hline 23 & -38 \\
\hline 24 & -42 \\
\hline 25 & 235 \\
\hline 26 & 522 \\
\hline 27 & 1020 \\
\hline 28 & 1092 \\
\hline 29 & 1095 \\
\hline 30 & 1097 \\
\hline 31 & 1092 \\
\hline
\end{tabular}

26. Shutdowns Scheduled Over Mext Six Months (Type, Date, Duration):

27. If Currently Shutdown, Estimated Startup Date:

Notes:

CUMULATIVE UNIT CAPACITY FACTOR (MDC NET) IS CALCULATED WITH A WEIGHTED AVERAGE. 


\begin{tabular}{|c|c|c|c|c|c|c|c|c|c|}
\hline No. & Date & Type & Hours & Reason & Method & LER Number & System & Component & Cause and Corrective Action To Prevent Recurrenc \\
\hline 1 & $12 / 14 / 94$ & $\mathbf{F}$ & 58.4 & H & 3 & 94020 & & & $\begin{array}{l}\text { WESTERN PONER POOL TRANSMISSION SYSTEM VOLTAGE } \\
\text { TRANSIENT. }\end{array}$ \\
\hline 2 & $12 / 19 / 94$ & $\mathbf{F}$ & 132.7 & H & 2 & 94012 & NN & & $\begin{array}{l}\text { MANUAL TRIP DUE TO HIGH CIRCULATING WATER } \\
\text { DIFFERENTIAL PRESSURE CAUSED BY HEAVY SEAS. }\end{array}$ \\
\hline
\end{tabular}

\begin{tabular}{|c|c|}
\hline TYPE & REASON \\
\hline $\begin{array}{l}\text { F: Forced } \\
\text { S: Scheduled }\end{array}$ & $\begin{array}{l}\text { A-Equipment Failure } \\
\text { B-Maintenance or Test } \\
\text { C-Refuel ing } \\
\text { D-Regulatory Restriction } \\
\text { E-Operator Training \& } \\
\text { License Examination } \\
\text {-Administrative } \\
\text { G-Operat ional Error } \\
\text { H-Other }\end{array}$ \\
\hline
\end{tabular}

\begin{tabular}{l} 
METHOD \\
\hline 1-Manual \\
2-Manual Scram \\
3-Auto Scram \\
4-Cont inued \\
5-Reduced Load \\
9-other
\end{tabular}

SYSTEM

IEEE Standard 805-1984 and/or

NUREG-0161 Exhibit F
COMPONENT

IEEE Standard

803A-1983 and/or

NUREG-0161 Exhibit H 


\section{FACILITY DESCRIPTION}

LOCATION

STATE................. CALIFORNIA
COUNTY............... SAN LUIS OBISPO

DIST AND DIRECTION FROM

MEAREST POPULATION CTR..... 12 MI USW OF SAN LUIS OBISPO

TYPE OF REACTOR.

PUR

DATE INITIAL CRITICALITY

AUGUST 20, 1985

DATE INITIAL ELECTRICITY.

OCTOBER 20, 1985

DATE COMARCIAL OPERATE.

CONDENSER COOLING METHOD

MARCH 12, 1986

CONDENSER COOLING WATER.

ELECTRIC RELIABILITY

COUNCIL.................. WESTERN SYSTEMS COORDIMATION COUNCIL

\section{UTILITY \& CONTRACTOR INFORMATION}

UTILITY

LICENSEE.................. PACIFIC gAS \& EleCtric co.

CORPORATE ADDRESS.......... 77 BEALE STREET

SAN FRANCISCO, CALIFORUIA 94106

\section{CONTRACTOR}

ARCHI TECT/ENGIMEER.

PACIFIC GAS \& ELECTRIC

MUC STEAM SYS SUPPLIER...... WESTINGHOUSE

CONSTRUCTOR

PACIFIC GAS \& ELECTRIC

TURBINE SUPPLIER.

MESTIMGHOUSE

REGULATORY INFORMATION

IE REGION RESPONSIBLE........ 4

IE RESIDENT INSPECTOR.

MICHAEL TSCHILTZ

LICENSING PROJ MANAGER

SHERI R. PETERSON

DOCKET MUMBER

50-323

LICENSE \& DATE ISSUANCE.

DPR 082, AUGUST 26, 1985 
1. Docket: $50-237$

OPERATING STATUS

2. Reporting Period: DECEMBER 1994 Outage + On-Line Hrs: 744.0

3. Utility Contact: K. H. SYKES (815) 942-2920 EXT. 2704

4. Licensed Thermal Power (MUt):

5. Nameplate Rating (Gross MHe):

6. Design Electrical Rating (Net MUe):

7. Maximum Dependable Capacity (Gross MWe):

8. Maximum Dependable Capacity (Net MWe):

9. If Changes Occurred Above Since Last Report, Give Reasons:

10. Power Level To Which Restricted, If Any (Net MWe):

11. Reasons For Restrictions, If Any:

\begin{tabular}{|c|c|c|c|}
\hline 2. Report Period Hrs & $\begin{array}{l}\text { MONTH } \\
744.0\end{array}$ & $\begin{array}{l}\text { YEAR } \\
8,760.0\end{array}$ & $\begin{array}{l}\text { CUMULATIVE } \\
215,952.0\end{array}$ \\
\hline - Hours Reactor Critical & 670.0 & $5,980.6$ & $160,438.5$ \\
\hline - Rx Reserve Shtdun Hrs & 0.0 & 0.0 & 0.0 \\
\hline - Hrs Generator On-Line & 628.0 & $5,808.6$ & $154,025.2$ \\
\hline - Unit Reserve Shtdwn Hrs & 0.0 & 0.0 & 0.0 \\
\hline Gross Therm Ener (MUH) & $1,304,121.0$ & $13,642,971.0$ & $319,634,259.0$ \\
\hline - Gross Elec Ener (MHH) & $413,236.0$ & $4,286,711.0$ & $101,898,122.0$ \\
\hline - Net Elec Ener (MUH) & $392,787.0$ & $4,069,330.0$ & $96,343,139.0$ \\
\hline - Unit Service Factor & 84.4 & 66.3 & 71.3 \\
\hline Unit Avail Factor & 84.4 & 66.3 & 71.3 \\
\hline - Unit Cap Factor (MDC Net) & 68.4 & 60.2 & 57.8 \\
\hline - Unit Cap Factor (DER Net) & 66.5 & 58.5 & 56.2 \\
\hline Unit Forced Outage Rate & 15.6 & 33.7 & 13.3 \\
\hline Forced Outage Hours & 116.0 & 2951.4 & $23,590.8$ \\
\hline
\end{tabular}

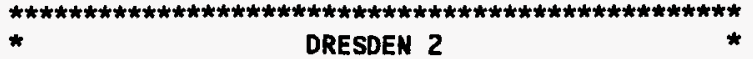

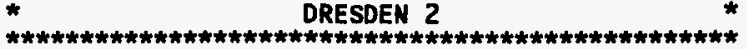

AVERAGE DAILY POWER LEVEL (Net MWe)

\begin{tabular}{|c|c|c|c|}
\hline DAY & POWER & DAY & POWER \\
\hline 1 & 0 & 16 & 745 \\
\hline 2 & 0 & 17 & 746 \\
\hline 3 & 0 & 18 & 668 \\
\hline 4 & 0 & 19 & 746 \\
\hline 5 & 42 & 20 & 746 \\
\hline 6 & 144 & 21 & 747 \\
\hline 7 & 162 & 22 & 740 \\
\hline 8 & 163 & 23 & 736 \\
\hline 9 & 407 & 24 & 514 \\
\hline 10 & 694 & 25 & 487 \\
\hline 11 & 733 & 26 & 564 \\
\hline 12 & 745 & 27 & 674 \\
\hline 13 & 749 & 28 & 743 \\
\hline 14 & 751 & 29 & 747 \\
\hline 15 & 748 & 30 & 746 \\
\hline & & 31 & 737 \\
\hline
\end{tabular}

26. Shutdowns Scheduled Over Next Six Months (Type, Date, Duration):

27. If Currently Shutdown, Estimated Startup Date:

Notes: 


\begin{tabular}{|c|c|c|c|c|c|c|c|c|c|}
\hline No. & Date & Type & Hours & Reason & Method & LER Number & System & Component & Cause and Corrective Action to Prevent Recurrence \\
\hline
\end{tabular}
SHUTDOWN WAS INITIATED DUE TO PROBLEMS WITH THE STH STOP CHECK VALVE.

\begin{tabular}{l} 
TYPE \\
\hline F: Forced \\
Scheduled
\end{tabular}

$\begin{array}{lll}\text { REASON } & \text { METHOD } \\ \text { A-Equipment Failure } & & \text { 1-Manual } \\ \text { B-Maintenance or Test } & & \text { 2-Manual Scram } \\ \text { C-Refuel ing } & & \text { 3-Auto Scram } \\ \text { D-Regulatory Restriction } & & \text { 4-Cont inued } \\ \text { E-Operator Training \& } & & 5 \text {-Reduced Load } \\ \text { License Examination } & & 9 \text {-Other } \\ \text { F-Administrative } & & \end{array}$

\section{SYSTEM}

IEEE Standard

NUREG-0161 Exhibit F

\section{COMPONENT}

\section{IEEE Standard}

NUREG-0161 Exhibit H 


\section{FACILITY DESCRIPTION}

\section{LOCATION}

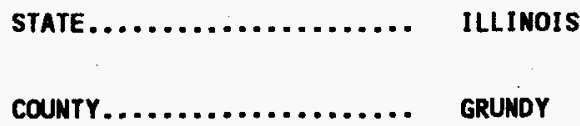

\section{DIST AND DIRECTION FROM}

NEAREST POPULATION CTR..... 9 MI E OF MORRIS, IL

TYPE OF REACTOR

DATE IMITIAL CRITICALITY......

DATE INITIAL ELECTRICITY......

DATE COMMERCIAL OPERATE.

CONDENSER COOLING METHOD......

CONDENSER COOLING MATER.

ELECTRIC RELIABILITY

COUNCIL..............

\section{UTILITY \& CONTRACTOR INFORMATION}

\section{UTILITY}

LICENSEE.

CORPORATE ADDRESS

\section{CONTRACTOR}

ARCHITECT/ENGINEER.......... SARGENT \& LUMDY

NUC STEAM SYS SUPPLIER......

GEMERAL ELECTRIC

CONSTRUCTOR

UNITED ENG. \& CONSTRUCTORS

TURBIME SUPPLIER.

\section{GENERAL ELECTRIC}

\section{REGULATORY IMFORMATION}

\author{
IE REGION RESPONSIBLE......... 3 \\ IE RESIDENT INSPECTOR......... MELVYN LEACH \\ LICEMSIMg PROJ MANAGER......... JOHN F. STAMG \\ DOCKET NUMBER............. 50-237 \\ LICENSE \& DATE ISSUANCE........ DPR 019, DECEMBER 22, 1969
}


1. Docket: $50-249$

OPERATING STATUS

2. Reporting Period: DECEMBER 1994

Outage + On-Line Hrs:

744.0

3. Utility Contact: K. H. SYKES (815) 942-2920 EXT. 2704

4. Licensed Thermal Power (MUt):

5. Nameplate Rating (Gross MWe):

6. Design Electrical Rating (Net MWe):

7. Maximum Dependable Capacity (Gross MHe):

8. Maximum Dependable Capacity (Het MWe):

\begin{tabular}{c}
\hline 2527 \\
\hline 840 \\
\hline 794 \\
\hline 840 \\
\hline 773
\end{tabular}

9. If Changes Occurred Above Since Last Report, Give Reasons:

10. Power Level To thich Restricted, If Any (Net MWe):

11. Reasons for Restrictions, If Any:

\begin{tabular}{|c|c|c|c|}
\hline 12. Report Period Hrs & $\begin{array}{l}\text { MONTH } \\
744.0\end{array}$ & $\begin{array}{l}\text { YEAR } \\
8,760.0\end{array}$ & $\begin{array}{l}\text { CUMULATIVE } \\
205,537.0\end{array}$ \\
\hline 3. Hours Reactor Critical & 744.0 & $3,085.0$ & $145,790.7$ \\
\hline 14. Rx Reserve Shtdwn Hrs & 0.0 & $\overline{0.0}$ & 0.0 \\
\hline j. Hrs Generator On-Line & 744.0 & $3,009.0$ & $140,323.6$ \\
\hline 5. Unit Reserve Shtdwn Mrs & 0.0 & $\overline{0.0}$ & 0.0 \\
\hline 7. Gross Therm Ener (MUH) & $1,789,358.0$ & $5,540,941.0$ & $289,275,758.0$ \\
\hline 8. Gross Elec Ener (MUH) & $579,164.0$ & $1,759,411.0$ & $93,110,296.0$ \\
\hline 9. Net Elec Ener (MHH) & $554,165.0$ & $1,624,540.0$ & $88,195,569.0$ \\
\hline . Unit Service Factor & 100.0 & 34.3 & 68.3 \\
\hline . Unit Avail Factor & 100.0 & $\overline{34.3}$ & 68.3 \\
\hline . Unit Cap Factor (mDC Net) & 96.4 & 24.0 & 55.5 \\
\hline Unit Cap Factor (DER Net) & 93.8 & 23.4 & 54.0 \\
\hline Unit Forced Outage Rate & 0.0 & 0.0 & 11.5 \\
\hline Forced Outage Hours & 0.0 & 0.0 & $18,265.8$ \\
\hline
\end{tabular}

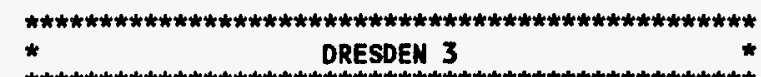

DRESDEN 3

AVERAGE DAILY POWER LEVEL (Net MWe)

\begin{tabular}{ll} 
DAY & PONER \\
\hline 1 & 689 \\
\hline 2 & 755 \\
\hline 3 & 738 \\
\hline 4 & 758 \\
\hline 5 & 760 \\
\hline 6 & 766 \\
\hline 7 & 772 \\
\hline 8 & 770 \\
\hline 9 & 772 \\
\hline 10 & 513 \\
\hline 11 & 686 \\
\hline 12 & 754 \\
\hline 13 & 767 \\
\hline 14 & 766 \\
\hline 15 & 767 \\
\hline
\end{tabular}

\begin{tabular}{ll} 
DAY & PONER \\
\hline 16 & 761 \\
\hline 17 & 753 \\
\hline 18 & 766 \\
\hline 19 & 765 \\
\hline 20 & 765 \\
\hline 21 & 765 \\
\hline 22 & 761 \\
\hline 23 & 759 \\
\hline 24 & 744 \\
\hline 25 & 721 \\
\hline 26 & 725 \\
\hline 27 & 758 \\
\hline 28 & 756 \\
\hline 29 & 760 \\
\hline 30 & 750 \\
\hline 31 & 733 \\
\hline
\end{tabular}

26. Shutdowns Scheduled Over Next Six Months (Type, Date, Duration):

27. If Currently Shutdown, Estimated Startup Date:

Notes: 


No. Date Type Hours Reason Method LER Mumber System Component Cause and Corrective Action To Prevent Recurrence

\section{METHOD}

1 -Manual

2-Manual Scram

3-Auto Scram

4-Cont inued

5-Reduced Load

9-0ther

\begin{tabular}{|c|c|}
\hline SYSTEM & COMPONENT \\
\hline $\begin{array}{l}\text { IEEE Standard } \\
805-1984 \text { and/or } \\
\text { NUREG-0161 Exhibit } F\end{array}$ & $\begin{array}{l}\text { IEEE Standard } \\
803 A-1983 \text { and/or } \\
\text { NUREG-0161 Exhibit } H\end{array}$ \\
\hline
\end{tabular}




\section{FACILITY DESCRIPTION}

\section{LOCATION}

STATE.................. ILLINOIS

COUNTY................. GRUNDY

\section{DIST AND DIRECTION FRO}

NEAREST POPULATION CTR..... 9 MI E OF MORRIS, IL

TYPE OF REACTOR.

BWR

DATE INITIAL CRITICALITY......

DATE INITIAL ELECTRICITY......

DATE COMMERCIAL OPERATE.

CONDENSER COOLING METHOD......

COMDENSER COOLING WATER

ELECTRIC RELIABILITY

COUNCIL.....................
JAMUARY 31, 1971

JULY 22, 1971

NOVEMBER 16, 1971

COOLING LAKE

KANKAKEE RIVER

MID-AMERICA INTERPOOL NETWORK
UTILITY \& CONTRACTOR INFORMATION

UTILITY

LICENSEE................ COMMONUEALTH EDISON CO.

CORPORATE ADDRESS......... 1400 OPUS PL., OPUS MEST III SUITE 300
DOWNER'S GROVE, ILLINOIS 60515

\section{CONTRACTOR}

ARCHITECT/ENGINEER

NUC STEAM SYS SUPPLIER

\section{SARGENT \& LUADY}

GEMERAL ELECTRIC

CONSTRUCTOR

UNITED ENG. \& CONSTRUCTORS

TURBINE SUPPLIER

GENERAL ELECTRIC

\section{REGULATORY INFORMATION}

IE REGION RESPONSIBLE......... 3

IE RESIDENT INSPECTOR

MELVYN LEACH

LICENSING PROJ MANAGER.

JOHN F. STANG

DOCKET NUMBER

$50-249$

LICENSE \& DATE ISSUANCE. 
1. Docket: $50-331$

OPERATING STATUS

2. Reporting Period: DECEMBER 1994

Outage + On-Line Hrs: $\mathbf{7 4 4 . 0}$

3. Utility Contact: RICHARD WOOOWARD (319) 851-7318

4. Licensed Thermal Power (MWt):

5. Nameplate Rating (Gross MWe):

6. Design Electrical Rating (Net MHe):

7. Maximum Dependable Capacity (Gross MHe):

8. Maximum Dependable Capacity (Net MHe):

9. If Changes Occurred Above Since Last Report, Give Reasons:

10. Power Level To Which Restricted, If Any (Net MWe):

11. Reasons for Restrictions, If Any:

\begin{tabular}{|c|c|c|c|}
\hline 12. Report Period Hrs & $\begin{array}{r}\text { MONTH } \\
744.0\end{array}$ & $\begin{array}{l}\text { YEAR } \\
8,760.0 \\
\end{array}$ & $\begin{array}{l}\text { CUMULATIVE } \\
174,576.0 \\
\end{array}$ \\
\hline 13. Hours Reactor Critical & 744.0 & $8,236.0$ & $131,180.6$ \\
\hline 14. Rx Reserve Shtdin Hrs & 0.0 & 0.0 & 192.8 \\
\hline 15. Hrs Generator On-Line & 743.8 & $8,080.4$ & $127,859.1$ \\
\hline 16. Unit Reserve Shtdwn Hrs & 0.0 & 0.0 & 0.0 \\
\hline 17. Gross Therm Ener (MUH) & $1,116,944.5$ & $13,069,795.6$ & $177,528,360.3$ \\
\hline 18. Gross Elec Ener (MMH) & $377,901.0$ & $4,367,501.0$ & $59,447,886.5$ \\
\hline 19. Net Elec Ener (MUH) & $355,493.6$ & $4,108,361.4$ & $55,740,024.2$ \\
\hline 20. Unit Service Factor & 100.0 & 92.2 & 73.2 \\
\hline 21. Unit Avail Factor & 100.0 & 92.2 & 73.2 \\
\hline 22. Unit Cap Factor (MDC Net) & 92.8 & 91.1 & 67.5 \\
\hline 23. Unit Cap Factor (DER Net) & 88.8 & 87.2 & 64.6 \\
\hline 24. Unit Forced Outage Rate & 0.0 & 2.4 & 11.4 \\
\hline 25. Forced Outage Hours & 0.0 & 202.8 & $16,388.1$ \\
\hline
\end{tabular}

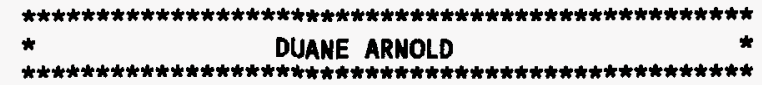

AVERAGE DAILY POWER LEVEL (Net MHe)

\begin{tabular}{ll} 
DAY & POUER \\
\hline 1 & 170 \\
\hline 2 & 458 \\
\hline 3 & 506 \\
\hline 4 & 527 \\
\hline 5 & 530 \\
\hline 6 & 531 \\
\hline 7 & 531 \\
\hline 8 & 531 \\
\hline 9 & 533 \\
\hline 10 & 475 \\
\hline 11 & 188 \\
\hline 12 & 194 \\
\hline 13 & 238 \\
\hline 14 & 510 \\
\hline 15 & 529 \\
\hline
\end{tabular}

\begin{tabular}{ll} 
DAY & PONER \\
16 & 531 \\
\hline 17 & 534 \\
\hline 18 & 535 \\
\hline 19 & 533 \\
\hline 20 & 531 \\
\hline 21 & 533 \\
\hline 22 & 535 \\
\hline 23 & 537 \\
\hline 24 & 534 \\
\hline 25 & 536 \\
\hline 26 & 534 \\
\hline 27 & 532 \\
\hline 28 & 535 \\
\hline 29 & 535 \\
\hline 30 & 533 \\
\hline 31 & 355 \\
\hline
\end{tabular}

26. Shutdowns Scheduled Over Next Six Months (Type, Date, Duration): REFUELING OUTAGE, FEBrUARY 23, 1995, 54 DAYS.

27. If Currently Shutdown, Estimated Startup Date: Notes:

CUMULATIVE UNIT CAPACITY FACTORS (MDC \& DER) ARE CALCULATED HITH WEIGHTED AVERAGES. 


\begin{tabular}{|c|c|c|c|c|c|c|c|c|c|}
\hline No. & Date & Type & Hours & Reason & Method & LER Number & System & Component & Cause and Corrective Action To Prevent Recurrenc \\
\hline 1 & $11 / 16 / 94$ & $\mathbf{s}$ & 0.3 & B & 4 & & SB & RV & PREVIOUS OUTAGE CONTINUED. \\
\hline 3 & $12 / 10 / 94$ & $\mathbf{F}$ & 0.0 & B & 5 & & AD & MG & $\begin{array}{l}\text { REDUCED PONER DURING SINGLE RECIRCULATION LOOP } \\
\text { OPERATION FOLLOWING RECIRCULATION PUMP TRIP. }\end{array}$ \\
\hline 4 & $12 / 31 / 94$ & $\mathbf{s}$ & 0.0 & B & 5 & & KH & ISV & $\begin{array}{l}\text { REDUCED PONER TO PERFORM REPAIR ON CONDENSATE } \\
\text { DEMINERALIZER INFLUENT ISOLATION VALVE. }\end{array}$ \\
\hline
\end{tabular}

\begin{tabular}{|c|c|}
\hline TYPE & REASOM \\
\hline $\begin{array}{l}\text { F: Forced } \\
\text { S: Scheduled }\end{array}$ & $\begin{array}{l}\text { A-Equipment Failure } \\
\text { B-Maintenance or Test } \\
\text { C-Refuel ing } \\
\text { D-Regulatory Restriction } \\
\text { E-Operator Training \& } \\
\text { License Examination } \\
\text { F-Administrat ive } \\
\text { G-Operational Error } \\
\text { H-Other }\end{array}$ \\
\hline
\end{tabular}

\begin{tabular}{l} 
METHOD \\
\hline 1-Manual \\
2-Manual Scram \\
3-Auto Scram \\
4-Cont inued \\
5-Reduced Load \\
9-0ther
\end{tabular}

\section{SYSTEM}

IEEE Standard

805-1984 and/or

NUREG-0161 Exhibit $F$

\section{COMPONENT}

IEEE Standard

$803 A-1983$ and/or

NUREG-0161 Exhibit 


\section{FACILITY DESCRIPIION}

\section{LOCATION}

STATE

COUNTY

IOWA

LINN

\section{DIST AND DIRECTION FROM}

NEAREST POPULATION CTR..... 8 MI NH OF CEDAR RAPIDS, IA

TYPE OF REACTOR.

DATE INITIAL CRITICALITY...... MARCH 23, 1974

OATE IHITIAL ELECTRICITY...... MAY i9, 1974

DATE COMMERCIAL OPERATE....... FEBRUARY 01, 1975

CONDENSER COOLING METHOD...... COOLING TOWER

CONDENSER COOLING WATER

ELECTRIC RELIABILITY

COUNCIL.................. MID-CONTINENT AREA RELIABILITY

\section{UTILITY \& CONTRACTOR INFORMATION}

UTILITY

LICENSEE.................. IOWA EleCTRIC LIGHT \& PONER CO.

CORPORATE ADDRESS........... P.0. BOX 351

CEDAR RAPIDS, IOWA 52406

\author{
CONTRACTOR \\ ARCHITECT/ENGINEER.......... BECHTEL \\ MUC STEAM SYS SUPPLIER...... GENERAL ELECTRIC \\ CONSTRUCTOR.............. BECHTEL \\ TURBIME SUPPLIER.......... GENERAL ELECTRIC
}

\section{REGULATORY INFORMATION}

IE REGION RESPONSIBLE......... 3

IE RESIDENT IHSPECTOR......... JAY HOPKINS

LICENSING PROJ MANAGER......... ROBERT M. PULSIFER

DOCKET MUMBER.............. 50-331

LICENSE \& DATE ISSUANCE....... DPR 049, FEBRUARY 22, 1974 


\section{Docket: $50-348$}

OPERATING STATUS

2. Reporting Period: DECEMBER 1994

Outage + On-Line Hrs: $\mathbf{7 4 4 . 0}$

3. Utility Contact: R. D. HILL (205) 899-5156

4. Licensed Thermal Power (MWt):

5. Nameplate Rating (Gross MHe):

6. Design Electrical Rating (Net MHe):

7. Maximum Dependable Capacity (Gross MUe):

8. Maximum Dependable Capacity (Net MWe):

9. If Changes Occurred Above Since Last Report, Give Reasons:

10. Power Level To Which Restricted, If Any (Net MWe):

11. Reasons for Restrictions, If Any:

\begin{tabular}{|c|c|c|c|}
\hline & MONTH & YEAR & CUMULATIVE \\
\hline 12. Report Period Hrs & 744.0 & $8,760.0$ & $149,760.0$ \\
\hline 13. Hours Reactor Critical & 744.0 & $7,592.9$ & $119,256.8$ \\
\hline 14. Rx Reserve Shtdwn Hrs & 0.0 & 0.0 & $3,650.0$ \\
\hline 15. Hrs Generator On-Line & 744.0 & $7,547.3$ & 117.453 .9 \\
\hline 16. Unit Reserve Shtdwn Hrs & 0.0 & 0.0 & 0.0 \\
\hline 17. Gross Therm Ener (MUH) & $1,972,719.6$ & $19,643,311.8$ & $302,135,716.8$ \\
\hline 18. Gross Elec Ener (МUH) & $649,957.0$ & $6,398,659.0$ & $97,413,605.0$ \\
\hline 19. Net Elec Ener (MWH) & $618,121.0$ & $6,059,835.0$ & $91,999,989.0$ \\
\hline 20. Unit Service Factor & 100.0 & 86.2 & 78.4 \\
\hline 21. Unit Avail Factor & 100.0 & 86.2 & 78.4 \\
\hline 22. Unit Cap Factor (MDC Net) & 102.3 & 85.2 & 75.4 \\
\hline 23. Unit Cap Factor (DER Net) & 100.2 & 83.4 & 74.1 \\
\hline 24. Unit Forced Outage Rate & 0.0 & 0.0 & 6.0 \\
\hline 25. Forced Outage Hours & 0.0 & 0.0 & $7,482.3$ \\
\hline
\end{tabular}

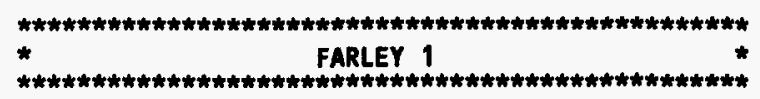

AVERAge dAILY PONER LeVel (Net MHe)

\begin{tabular}{ll} 
DAY & PONER \\
\hline 1 & 834 \\
\hline 2 & 831 \\
\hline 3 & 827 \\
\hline 4 & 827 \\
\hline 5 & 825 \\
\hline 6 & 829 \\
\hline 7 & 829 \\
\hline 8 & 829 \\
\hline 9 & 828 \\
\hline 10 & 826 \\
\hline 11 & 833 \\
\hline 12 & 834 \\
\hline 13 & 834 \\
\hline 14 & 833 \\
\hline 15 & 833 \\
\hline
\end{tabular}

\begin{tabular}{cc} 
DAY & PONER \\
\hline 16 & 831 \\
\hline 17 & 832 \\
\hline 18 & 832 \\
\hline 19 & 834 \\
\hline 20 & 833 \\
\hline 21 & 831 \\
\hline 22 & 832 \\
\hline 23 & 830 \\
\hline 24 & 832 \\
\hline 25 & 833 \\
\hline 26 & 833 \\
\hline 27 & 832 \\
\hline 28 & 832 \\
\hline 29 & 831 \\
\hline 30 & 830 \\
\hline 31 & 831 \\
\hline
\end{tabular}

26. Shutdowns Scheduled Over Next Six Months (Type, Date, Duration):

27. If Currently Shutdown, Estimated Startup Date:

Notes:

CUMULATIVE UNIT CAPACITY FACTOR (MDC MET) IS CALCULATED MITH A MEIGHTED AVERAGE. 


No. Date Type Hours Reason Method LER Number System Component Cause and Corrective Action To Prevent Recurrence

\begin{tabular}{|c|c|}
\hline TYPE & REASON \\
\hline $\begin{array}{l}\text { F: Forced } \\
\text { S: Scheduled }\end{array}$ & $\begin{array}{l}\text { A-Equipment Failure } \\
\text { B-Maintenance or Test } \\
\text { C-Refuel ing } \\
\text { D-Regulatory Restriction } \\
\text { E-Operator Training \& } \\
\text { License Examination } \\
\text { F-Administrat ive } \\
\text { G-Operational Error } \\
\text { H-other }\end{array}$ \\
\hline
\end{tabular}

\begin{tabular}{l} 
METHOD \\
\hline 1-Manual \\
2-Manual Scram \\
3--Auto Scram \\
4-Cont inued \\
5-Reduced Load \\
9-0ther
\end{tabular}

\section{SYSTEM}

IEEE Standard

NUREG-0161 Exhibit F

\section{COMPONENT}

IEEE Standard

803A-1983 and/or

NUREG-0161 Exhibit H

Administrat ive

H-other 
UTILITY \& CONTRACTOR INFORMATION

\section{FACILITY DESCRIPTIOH}

\section{LOCATION}

STATE.

ALABAMA

COUNTY

HOUSTON

\section{DIST AND DIRECTION FROM}

MEAREST P

TYPE OF REACTOR.

DATE INITIAL CRITICALITY...... AUGUST 09, 1977

DATE IMITIAL ELECTRICITY...... AUGUST 18, 1977

DATE COMmercial OPERATE....... DeCEMBER 01, 1977

CONDENSER COOLING METHOD...... COOLING TONERS

CONDENSER COOLING WATER.

CHATAHOOCHEE RIVER

ELECTRIC RELIABILITY

COUNCIL.................... SOUTHEASTERN ELECTRIC RELIABILITY COUNCIL

\section{UTILITY}

LICENSEE.

CORPORATE ADDRES

CONTRACTOR

ARCHITECT/ENGIMEER......... SOUTHERN SERVICES IMCORPORATED

MUC STEAM SYS SUPPLIER...... WESTINGHOUSE

CONSTRUCTOR .............. DANIEL IHTERMATIOMAL

TURBINE SUPPLIER.......... WESTINGHOUSE

\section{REGULATORY INFORMATION}

IE REGION RESPONSIBLE........ 2

IE RESIDENT INSPECTOR

THIERRY ROSS

LICENSING PROJ MANAGER.

BYRON L. SIEGEL

DOCKET NUMBER

$50-348$

LICENSE \& DATE ISSUANCE.

NPF 002, JUNE 25, 1977 


\section{Docket: $50-364$}

OPERATING STATUS

2. Reporting Period: DECEMBER 1994

Outage + On-Line Hrs: 744.0

3. Utility Contact: R. D. HILL (205) 899-5156

4. Licensed Thermal Power (MWt):

5. Nameplate Rating (Gross Whe):

6. Design Electrical Rating (Net MWe):

7. Maximum Dependable Capacity (Gross MWe):

8. Maximum Dependable Capacity (Net MUe):

9. If Changes Occurred Above Since Last Report, Give Reasons:

10. Power Level To Which Restricted, If Any (Net MWe):

11. Reasons For Restrictions, If Any:

\begin{tabular}{|c|c|c|c|}
\hline & MONTH & YEAR & CUMULATIVE \\
\hline 12. Report Period Hrs & 744.0 & $8,760.0$ & $117,673.0$ \\
\hline 13. Hours Reactor Critical & 702.0 & $8,704.0$ & $101,657.7$ \\
\hline 14. RX Reserve Shtown Hirs & $0 . \overline{0}$ & $0 . \overline{0}$ & $\mathbf{1 3 8} \overline{8} .0$ \\
\hline 15. Hrs Generator On-Line & 676.0 & $8,660.8$ & $100,222.6$ \\
\hline 16. Unit Reserve Shtdwn Hrs & 0.0 & 0.0 & 0.0 \\
\hline 17. Gross Therm Ener (MWH) & $1,709,587.1$ & $22,804,352.9$ & $256,339,704.2$ \\
\hline 18. Gross Elec Ener (MUH) & $562,813.0$ & $7,505,081.0$ & $84,054,520.0$ \\
\hline 19. Net Elec Ener (MUH) & $532,685.0$ & $7,147,209.0$ & $79,722,298.0$ \\
\hline 20. Unit Service Factor & 90.9 & 98.9 & 85.2 \\
\hline 21. Unit Avail Factor & 90.9 & 98.9 & 85.2 \\
\hline 22. Unit Cap Factor (MDC Net) & 87.1 & 99.3 & 82.6 \\
\hline 23. Unit Cap Factor (DER Net) & 86.4 & 98.4 & 81.7 \\
\hline 24. Unit Forced Outage Rate & 9.1 & 1.1 & 4.0 \\
\hline 25. Forced Outage Hours & 68.0 & 99.2 & $4,144.8$ \\
\hline
\end{tabular}

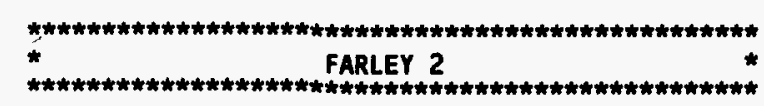

AVERAGE DAILY POMER LEVEL (Net MWe)

\begin{tabular}{ll} 
DAY & PONER \\
\hline 1 & 846 \\
\hline 2 & 843 \\
\hline 4 & 836 \\
\hline 5 & 831 \\
\hline 6 & 838 \\
\hline 7 & 838 \\
\hline 8 & 838 \\
\hline 9 & 837 \\
\hline 10 & 845 \\
\hline 11 & 845 \\
\hline 12 & 845 \\
\hline 13 & 843 \\
\hline 14 & 843 \\
\hline 15 & 843 \\
\hline
\end{tabular}

\begin{tabular}{cc} 
DAY & PONER \\
\hline 16 & 841 \\
\hline 17 & 840 \\
\hline 18 & 107 \\
\hline 19 & 8 \\
\hline 20 & 331 \\
\hline 21 & 820 \\
\hline 22 & 839 \\
\hline 23 & 839 \\
\hline 24 & 842 \\
\hline 25 & 535 \\
\hline 26 & 0 \\
\hline 27 & 334 \\
\hline 28 & 777 \\
\hline 29 & 837 \\
\hline 30 & 836 \\
\hline 31 & 839 \\
\hline
\end{tabular}

26. Shutdowns Scheduled Over Next Six Months (Type, Date, Duration): REFUELING/MAINTENANCE OUTAGE, MARCH 10, 1995, 39 DAYS.

27. If Currently Shutdown, Estimated Startup Date:

Notes:

CUMULATIVE UNIT CAPACITY FACTOR (MOC MET) IS CALCULATEO WITH A MEIGHTED AVERAGE. 


\begin{tabular}{|c|c|c|c|c|c|c|c|c|c|}
\hline No. & Date & Type & Hours & Reason & Method & LER Number & System & Component & Cause and Corrective Action To Prevent Recurrence \\
\hline 004 & $12 / 18 / 94$ & $\mathbf{F}$ & 38.2 & A & 3 & 94003 & & & $\begin{array}{l}\text { REACTOR TRIPPED DUE TO LON-LON WATER LEVEL IN THE } \\
\text { 2C STEAM GENERATOR (SG). THIS WAS A RESULT OF A } \\
\text { TRANSIENT INITIATED FROA THE CLOSING OF ALL FOUR } \\
\text { GOVERNOR VALVES DUE TO AN INTERMITTENT FAILURE OF } \\
\text { THE TURBIME CONTROL SYSTEM. }\end{array}$ \\
\hline 005 & $12 / 25 / 94$ & $\mathbf{F}$ & 29.8 & $\mathbf{A}$ & 3 & 94004 & & & $\begin{array}{l}\text { THE REACTOR TRIPPED DUE TO A TURBINE TRIP CAUSED } \\
\text { BY AN INTERMITTENT DEHC SYSTEM FAILURE. UHEN } \\
\text { COMPARED TO THE 12/18/94 REACTOR TRIP, BOTH TRIPS } \\
\text { MERE MOST LIKELY CAUSED BY AN INTERMITTENT DEHC } \\
\text { SYSTEM PROCESSOR FAILURE. DEHC CARDS WERE } \\
\text { REPLACED. }\end{array}$ \\
\hline
\end{tabular}

\begin{tabular}{|c|c|c|c|c|}
\hline TYPE & REASON & METHOD & SYSTEM & COMPONENT \\
\hline $\begin{array}{l}\text { F: Forced } \\
\text { s: Scheduled }\end{array}$ & $\begin{array}{l}\text { A-Equipment Failure } \\
\text { B-Maintenance or Test } \\
\text { C-Refuel ing } \\
\text { D-Regulatory Restriction } \\
\text { E-Operator Training \& } \\
\text { License Examination } \\
\text { F-Administrative } \\
\text { G-Operational Error } \\
\text { H-Other }\end{array}$ & $\begin{array}{l}\text { 1-Manual } \\
\text { 2-Manual Scram } \\
\text { 3-Auto Scram } \\
\text { 4-Cont inued } \\
5 \text {-Reduced Load } \\
9 \text {-0ther }\end{array}$ & $\begin{array}{l}\text { IEEE Standard } \\
805-1984 \text { and/or } \\
\text { NUREG-0161 Exhibit F }\end{array}$ & $\begin{array}{l}\text { IEEE Standard } \\
803 \mathrm{~A}-1983 \text { and/or } \\
\text { NUREG-0161 Exhibit H }\end{array}$ \\
\hline
\end{tabular}




\section{FACILITY DESCRIPTION}

LOCATION

STATE.

COUNTY.

DIST AND DIRECTION FROM

MEAREST POPULATION CTR

TYPE OF REACTOR.

DATE INITIAL CRITICALITY.

DATE INITIAL ELECTRICITY $=====$

DATE COMERCIAL OPERATE........

CONDENSER COOLING METHOD......

CONDENSER COOLING MATER.......

ELECTRIC RELIABILITY

COUNCIL.................. SOUTHEASTERN ELECTRIC RELIABILITY COUNCIL

\section{ALABAMA}

HOUSTON

18 MI SE OF DOTHAN, AL

PWR

MAY 08, 1981

MaY 25, 1981

JULY 30, 1981

COOLING TOWERS

CHATAHOOCHEE RIVER

\section{UTILITY}

LICENSEE.

CORPORATE ADDRESS.

\section{CONTRACTOR}

ARCHITECT/ENGINEER

NUC STEAM SYS SUPPLIER

CONSTRUCTOR

MESTIMGHOUSE

TURBINE SUPPLIER.......... WESTINGHOUSE

BECHTEL

\section{REGULATORY INFORMATION}

IE REGION RESPONSIBLE......... 2

IE RESIDENT INSPECTOR.......... THIERRY ROSS

LICENSING PROJ MANAGER......... BYRON L. SIEGEL

DOCKET NUMBER.............. 50-364

LICENSE \& DATE ISSUANCE........ MPF 008, MARCH 31, 1981 
1. Docket: $50-341$

OPERATING STATUS

2. Reporting Period: DECEMBER 1994

Outage + On-Line Hrs:

744.0

3. Utility Contact: B. J. STONE (313) 586-5148

4. Licensed Thermal Power (MWt):

5. Nameplate Rating (Gross MWe):

6. Design Electrical Rating (Net Me):

7. Maximum Dependable Capacity (Gross MUe):

8. Maximum Dependable Capacity (Net MHe):

9. If Changes Occurred Above since Last Report, Give Reasons:

10. Power Level To thich Restricted, If Any (Net MHe):

11. Reasons for Restrictions, If Any:

CURRENTLY LIMITING PONER TO 93.5\% CTP MAXIMUM TO LIMIT TURBINE COMPONENT VIBRATION. ALL RATINGS REFLECT 98\% CTP DUE TO TURBINE THROTTLE VALVE LIMITATIONS.

12. Report Period Hrs

13. Hours Reactor Critical

14. Rx Reserve Shtdwn Hrs

15. Hrs Generator On-Line

16. Unit Reserve Shtdwn Hrs

17. Gross Therm Ener (MUH)

18. Gross Elec Ener (MWH)

19. Net Elec Ener (MWH)

20. Unit Service Factor

21. Unit Avail Factor

22. Unit Cap Factor (MDC Net)

23. Unit Cap factor (DER Net)

24. Unit Forced Outage Rate

25. Forced Outage Hours
MONTH YEAR CUMULATIVE

\begin{tabular}{|c|c|c|}
\hline 744.0 & $8,760.0$ & $60,830.0$ \\
\hline 190.6 & 190.6 & $40,735.6$ \\
\hline 0.0 & 0.0 & 0.0 \\
\hline 0.0 & 0.0 & $39,130.9$ \\
\hline 0.0 & 0.0 & 0.0 \\
\hline $11,364.0$ & $11,364.0$ & $119,714,051.0$ \\
\hline 0.0 & 0.0 & $39,973,921.0$ \\
\hline 0.0 & 0.0 & $38,233,715.0$ \\
\hline 0.0 & 0.0 & 64.3 \\
\hline 0.0 & 0.0 & 64.3 \\
\hline 0.0 & 0.0 & 58.5 \\
\hline 0.0 & 0.0 & 57.1 \\
\hline 100.0 & 100.0 & 21.0 \\
\hline 744.0 & 7417.0 & $10,402.7$ \\
\hline
\end{tabular}

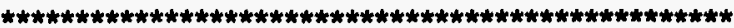

* FERMI 2 (

AVERAGE DAILY, POWER LEVEL (Net MHe)

\begin{tabular}{lc} 
DAY & PONER \\
\hline 1 & 0 \\
\hline 2 & 0 \\
\hline 3 & 0 \\
\hline 4 & 0 \\
\hline 5 & 0 \\
\hline 6 & 0 \\
\hline 7 & 0 \\
\hline 8 & 0 \\
\hline 9 & 0 \\
\hline 10 & 0 \\
\hline 11 & 0 \\
\hline 12 & 0 \\
\hline 13 & 0 \\
\hline 14 & 0 \\
\hline 15 & 0 \\
\hline
\end{tabular}

\begin{tabular}{cc} 
DAY & POWER \\
\hline 16 & 0 \\
\hline 17 & 0 \\
\hline 18 & 0 \\
\hline 19 & 0 \\
\hline 20 & 0 \\
\hline 21 & 0 \\
\hline 22 & 0 \\
\hline 23 & 0 \\
\hline 24 & 0 \\
\hline 25 & 0 \\
\hline 26 & 0 \\
\hline 27 & 0 \\
\hline 28 & 0 \\
\hline 29 & 0 \\
\hline 30 & 0 \\
\hline 31 & 0 \\
\hline
\end{tabular}

26. Shutdowns Scheduled Over Next Six Months (Type, Date, Duration):

27. If Currently Shutdown, Estimated Startup Date:

Notes:

CUMULATIVE UNIT CAPACITY FACTORS (MDC \& DER) ARE CALCULATED WITH WEIGHTED AVERAGES. 
POUER REDUCTIONS

\begin{tabular}{|c|c|c|c|c|c|c|c|c|c|}
\hline No. & Date & Type & Hours & Reason & Method & LER Number & System & Component & Cause and Corrective Action To Prevent Recurrenc \\
\hline $593-06$ & $12 / 25 / 93$ & $\mathbf{F}$ & 744.0 & A & 4 & 93014 & TA & TRB & $\begin{array}{l}\text { REACTOR TRIPPED FOLLONING TRIP OF MAIN TURBINE. } \\
\text { TURBINE TRIPPED DUE TO IMBALANCE CAUSED BY LOSS } \\
\text { OF } 5 \text { 8TH STAGE BLADES IN LON PRESSURE TURBINE } \\
\text { NUMBER } 3 \text {. OPERATION WITHOUT 7TH AND 8TH STAGES } \\
\text { IN ALL } 3 \text { LP TURBINES IS PLANNED FOR UPCOMING } \\
\text { OPERATING CYCLE. }\end{array}$ \\
\hline
\end{tabular}

TYPE

F: Forced

S: Scheduled

\section{REASON}

A-Equipment Failure B-Maintenance or Test C-Refuel ing

D-Regulatory Restriction

E-operator Training \&

License Examination

F-Administrat ive

G-Operational Error

H-Other

\begin{tabular}{l} 
METHOD \\
\hline 1-Manual \\
2-Manual Scram \\
3-Auto Scram \\
4-Cont inued \\
5-Reduced Load \\
9-0ther
\end{tabular}

SYSTEM

IEEE Standard

805-1984 and/or

NUREG-0161 Exhibit F
COMPONENT

IEEE Standard

803A-1983 and/or

NUREG-0161 Exhibit H 


\section{FACILITY DESCRIPTION}

\section{LOCATION}

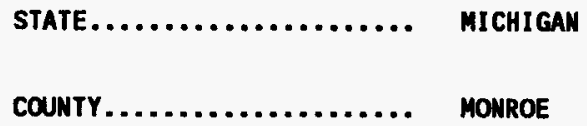

\section{DIST AND DIRECTION FRON}

NEAREST POPULATION CTR..... 25 MI NE OF TOLEDO, OH

TYPE OF REACTOR.

BWR

DATE INITIAL CRITICALITY...... JUNE 21, 1985

DATE IMITIAL ELECTRICITY...... SEPTEMBER 21, 1986

DATE COMMERCIAL OPERATE...... Jamuary 23,1988

CONDENSER COOLING METHOD...... ONCE THRU

CONDENSER COOLING WATER...... LAKE ERIE

ELECTRIC RELIABILITY

COUNCIL................. EAST CENTRAL AREA RELIABILITY

\section{UTILITY \& CONTRACTOR IMFORMATION}

UTILITY

LICENSEE.................. DETROIT EDISON CO.

CORPORATE ADDRESS......... 2000 SECOND AVENUE DETROIT, MICHIGAN 48226

\section{CONTRACTOR}

ARCHI TECT/ENGINEER.

SARGENT \& LUNDY

NUC STEAM SYS SUPPLIER

GENERAL ELECTRIC

CONSTRUCTOR.

DANIEL INTERMATIONAL

TURBINE SUPPLIER

DETROIT EDISON

RECULATORY INFORMATION

IE REGION RESPONSIBLE......... 3

IE RESIOENT INSPECTOR.......... ANTON VEGEL

LICENSING PROJ MANAGER......... TIMOTHY G. COLBURN

DOCKET MUMBER.............. 50-341

LICENSE \& DATE ISSUANCE........ NPF 043, JULY 16, 1985 
1. Docket: $50-333$

OPERATING STATUS

2. Reporting Period: DECEMBER 1994

Outage + On-Line Hrs: $\mathbf{7 4 4 . 0}$

3. Utility Contact: RUSSELL FLAGG (315) 349-6768

4. Licensed Thermal Power (MWt):

5. Nameplate Rating (Gross MWe):

6. Design Electrical Rating (Net MHe):

7. Maximum Dependable Capacity (Gross MHe):

8. Maximum Dependable Capacity (Net MHe):

9. If Changes Occurred Above Since Last Report, Give Reasons:

10. Power Level To thich Restricted, If Any (Net MWe):

11. Reasons For Restrictions, If Any:

\begin{tabular}{|c|c|c|c|}
\hline & MONTH & YEAR & CUMULATIVE \\
\hline 12. Report Period Hrs & 744.0 & $8,760.0$ & $170,329.0$ \\
\hline 13. Hours Reactor Critical & 0.0 & $7,291.9$ & $119,280.7$ \\
\hline 14. Rx Reserve Shtdun Hrs & 0.0 & 0.0 & 0.0 \\
\hline 15. Hrs Generator on-Line & 0.0 & $7,225.4$ & $115,260.5$ \\
\hline 16. Unit Reserve Shtdwn Hrs & 0.0 & 0.0 & 0.0 \\
\hline 17. Gross Therm Ener (MUH) & 0.0 & $15,428,232.0$ & $254,209,267.0$ \\
\hline 18. Gross Elec Ener (MHH) & 0.0 & $5,154,810.0$ & $86,392,810.0$ \\
\hline 19. Net Elec Ener (MWH) & 0.0 & $4,972,050.0$ & $82,987,875.0$ \\
\hline 20. Unit Service factor & 0.0 & 82.5 & 67.7 \\
\hline 21. Unit Avail Factor & 0.0 & 82.5 & 67.7 \\
\hline 22. Unit Cap Factor (MDC Net) & 0.0 & 73.3 & 66.2 \\
\hline 23. Unit Cap Factor (DER Net) & 0.0 & 69.6 & 59.7 \\
\hline 24. Unit Forced Outage Rate & 0.0 & 0.0 & $\overline{12.2}$ \\
\hline 25. Forced Outage Hours & 0.0 & 0.0 & $16,036.5$ \\
\hline
\end{tabular}

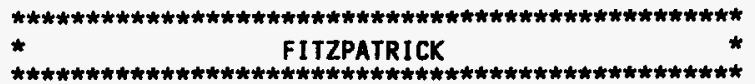

AVERAGE DAILY POWER LEVEL (Net MiNe)

\begin{tabular}{lr} 
DAY & PONER \\
\hline 1 & 0 \\
\hline 2 & 0 \\
\hline 3 & 0 \\
\hline 4 & 0 \\
\hline 5 & 0 \\
\hline 6 & 0 \\
\hline 7 & 0 \\
\hline 8 & 0 \\
\hline 9 & 0 \\
\hline 10 & 0 \\
\hline 11 & 0 \\
\hline 12 & 0 \\
\hline 13 & 0 \\
\hline 14 & 0 \\
\hline 15 & 0 \\
\hline
\end{tabular}

\begin{tabular}{cc} 
DAY & PONER \\
\hline 16 & 0 \\
\hline 17 & 0 \\
\hline 18 & 0 \\
\hline 19 & 0 \\
\hline 20 & 0 \\
\hline 21 & 0 \\
\hline 22 & 0 \\
\hline 23 & 0 \\
\hline 24 & 0 \\
\hline 25 & 0 \\
\hline 26 & 0 \\
\hline 27 & 0 \\
\hline 28 & 0 \\
\hline 29 & 0 \\
\hline 30 & 0 \\
\hline 31 & 0 \\
\hline
\end{tabular}

26. Shutdowns Scheduled Over Next Six Months (Type, Date, Duration):

27. If Currently Shutdown, Estimated Startup Date: 02/08/95

Notes:

CUMULATIVE UNIT CAPACITY FACTOR (MDC NET) IS CALCULATED WITH A WEIGHTED AVERAGE. 
Report Period DECEMBER 1994

\begin{abstract}
U N I T
\end{abstract}
S H U T D OUM

A N D

P O W E

REDUCTIONS

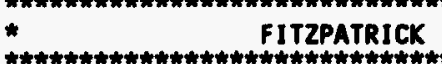

\begin{tabular}{cccccccccc}
\hline No. & Date & Type & Hours & Reason & Method & LER Number & system & Component & Cause and Corrective Action To Prevent Recurrence \\
\hline 2 & $11 / 30 / 94$ & S & 744.0 & C & 4 & RC & FUELXX & REFUELING OUTAGE CONTINUED.
\end{tabular}

REFUELING OUTAGE CONTINUED.

TYPE
F: Forced
S: Scheduled

\begin{tabular}{|c|c|}
\hline REASON & METHOD \\
\hline $\begin{array}{l}\text { A-Equipment Failure } \\
\text { B-Maintenance or Test } \\
\text { C-Refuel ing } \\
\text { D-Regulatory Restriction } \\
\text { E-Operator Training \& } \\
\text { License Examination }\end{array}$ & $\begin{array}{l}\text { 1-Manual } \\
\text { 2-Manual Scram } \\
\text { 3-Auto Scram } \\
\text { 4-Cont inued } \\
\text { 5-Reduced Load } \\
\text { 9-0ther }\end{array}$ \\
\hline
\end{tabular}

License Examination

G-Operational Error

-other

5-Reduced Load

9-0ther
COMPONENT
SYSTEN

IEEE Standard

MUREG-0161 Exhibit
IEEE Standard

$803 \mathrm{~A}-1983$ and/or

NUREG-0161 Exhibit H 


\section{FACILITY DESCRIPTION}

\section{LOCATION}

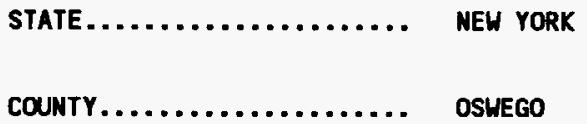

\section{DIST AND DIRECTION FRO}

MEAREST POPULATION CTR

TYPE OF REACTOR.

8 MI NE OF OSHEGO, NY

DATE INITIAL CRITICALITY...... NOVEMBER 17, 1974

DÂTE INITTIAL ELECTRICITYY......

DATE COMMERCIAL OPERATE.

CONDENSER COOLING METHOD

CONDENSER COOLING WATER.

ELECTRIC RELIABILITY

COUNCIL................... $\begin{aligned} & \text { NORTHEASTERH POWER } \\ & \text { COORDINATION COUNCIL }\end{aligned}$

\section{UTILITY \& CONTRACTOR INFORMATION}

UTILITY

LICENSEE. . . ............... NEW YORK PONER AUTHORITY

CORPORATE ADDRESS.......... 10 COLUMBUS CIRCLE

NEW YORK, NEW YORK 10019

\section{CONTRACTOR}

ARCHI TECT/ENGINEER.

STONE \& WEBSTER

NUC STEAM SYS SUPPLIER.

GENERAL ELECTRIC

CONSTRUCTOR.

STONE \& WEBSTER

TURBINE SUPPLIER

GENERAL ELECTRIC

\section{REGULATORY INFORMATION}

IE REGION RESPONSIBLE........ 1
IE RESIDENT INSPECTOR........ HILLIAM ANDREH COOK
LICENSING PROJ MANAGER....... JOHN E. MENNING
DOCKET MUMBER............... $50-333$
LICENSE \& DATE ISSUANCE....... DPR 059, OCTOBER 17,1974


1. Docket: $50-285$

OPERATING STATUS

2. Reporting Period: DECEMBER 1994

Outage + On-Line Hrs: $\quad 744.0$

3. Utility Contact: T. C. MATTHEWS (402) 553-6938

4. Licensed Thermal Power (MWt):

5. Nameplate Rating (Gross MWe):

6. Design Electrical Rating (Net MWe):

7. Maximum Dependable Capacity (Gross MWe):

8. Maximum Dependable Capacity (Net MWe):

9. If Changes Occurred Above since Last Report, Give Reasons:

10. Power Level To which Restricted, If Any (Net MWe):

11. Reasons For Restrictions, If Any:

\begin{tabular}{|c|c|c|c|}
\hline & MONTH & YEAR & CUMULATIVE \\
\hline 12. Report Period Hrs & 744.0 & $8,760.0$ & $186,434.0$ \\
\hline 13. Hours Reactor Critical & 744.0 & $8,726.2$ & $146,417.9$ \\
\hline 14. Rx Reserve Shtdwn Hrs & 0.0 & 0.0 & $1,309.5$ \\
\hline 15. Hrs Generator on-Line & 744.0 & $8,711.1$ & $144,774.1$ \\
\hline 16. Unit Reserve Shtdun Hrs & 0.0 & 0.0 & 0.0 \\
\hline 17. Gross Therm Ener (MUH) & $1,113,276.2$ & $12,862,900.9$ & $192,148,700.5$ \\
\hline 18. Gross Elec Ener (MHH) & $378,780.0$ & $4,316,858.0$ & $63,404,882.2$ \\
\hline 19. Net Elec Ener (MUH) & $362,103.6$ & $4,118,729.4$ & $60,491,792.3$ \\
\hline 20. Unit Service Factor & 100.0 & 99.4 & 77.7 \\
\hline 21. Unit Avail Factor & 100.0 & 99.4 & 77.7 \\
\hline 22. Unit Cap Factor (MDC Net) & 101.8 & 98.4 & 70.2 \\
\hline 23. Unit Cap Factor (DER Net) & 101.8 & 98.4 & 68.6 \\
\hline 24. Unit Forced Outage Rate & 0.0 & 0.6 & 4.0 \\
\hline 25. Forced Outage Hours & 0.0 & 48.9 & $5,980.0$ \\
\hline
\end{tabular}

\section{***************************************************

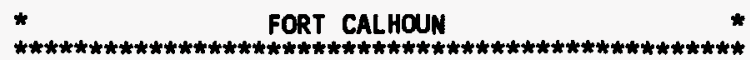

AVERAGE DAILY POUER LEVEL (Net MWe)

\begin{tabular}{ll} 
DAY & POWER \\
\hline 1 & 486 \\
\hline 2 & 487 \\
\hline 3 & 486 \\
\hline 4 & 486 \\
\hline 5 & 486 \\
\hline 6 & 486 \\
\hline 7 & 486 \\
\hline 8 & 486 \\
\hline 9 & 486 \\
\hline 10 & 487 \\
\hline 11 & 487 \\
\hline 12 & 487 \\
\hline 13 & 487 \\
\hline 14 & 487 \\
\hline 15 & 487 \\
\hline
\end{tabular}

\begin{tabular}{ll} 
DAY & POWER \\
\hline 16 & 487 \\
\hline 17 & 487 \\
\hline 18 & 487 \\
\hline 19 & 487 \\
\hline 20 & 487 \\
\hline 21 & 487 \\
\hline 22 & 487 \\
\hline 23 & 487 \\
\hline 24 & 487 \\
\hline 25 & 487 \\
\hline 26 & 487 \\
\hline 27 & 487 \\
\hline 28 & 487 \\
\hline 29 & 487 \\
\hline 30 & 487 \\
\hline 31 & 487 \\
\hline
\end{tabular}

26. Shutdowns Scheduled Over Mext Six Months (Type, Date, Duration): REFUELING OUTAGE, MARCH 11, 1995, 49 DAYS.

27. If Currently Shutdown, Estimated Startup Date:

Notes:

CUMULATIVE UNIT CAPACITY FACTORS (MDC \& DER) ARE CALCULATED WITH WEIGHTED AVERAGES. 
Report Period DECEMBER 1994 U N I

\begin{tabular}{l} 
TYPE \\
\hline F: Forced \\
S: Scheduled
\end{tabular}

\begin{tabular}{l} 
REASON \\
\hline A-Equipment Failure \\
B-Maintenance or Test \\
C-Refuel ing \\
D-Regulatory Restriction \\
E-Operator Training \& \\
License Examination \\
F-Administrat ive \\
G-Operat ional Error
\end{tabular}

H-other

\begin{tabular}{l} 
METHCD \\
\hline 1-Manual \\
2-Manual Scram \\
3-Auto Scram \\
4-Cont inued \\
5-Reduced Load \\
9-0ther
\end{tabular}

SYSTEM

IEEE Standard

805-1984 and/or

NUREG-0161 Exhibit F

COMPONENT

IEEE Standard

803A-1983 and/or

NUREG-0161 Exhibit H 


\section{FACILITY DESCRIPTION}

\section{LOCATION}

STATE

COUNTY

\section{DIST AND DIRECTION FRON}

MEAREST POPULATION CTR..... 19 MI N OF OMAHA, ME

TYPE OF REACTOR

PWR

DATE INITIAL CRITICALITY...... AUGUST 06, 1973

DATE INITIAL ELECTRICITY...... AUGUST 25, 1973

DATE COMmERCial operate....... JUNE 20, 1974

CONDENSER COOLING METHOD...... ONCE THRU

CONDENSER COOLING WATER....... MISSOURI RIVER

ELECTRIC RELIABILITY

COUNCIL.................. MID-CONTINENT AREA RELIABILITY COUNCIL

\section{UTILITY \& CONTRACTOR INFORMATION}

\section{UTILITY}

LICENSEE

CORPORATE ADDRESS.

OMAHA PUBLIC PONER DISTRICT

1623 HARNEY STREET

OMAHA, MEBRASKA 68102

\section{CONTRACTOR}

ARCHITECT/ENGINEER.

NUC STEAM SYS SUPPLIER

CONSTRUCTOR

TURBINE SUPPLIER.

GIBBS, HILL, DURHAM \& RICHARDSON

COMBUSTION ENGINEERING

GIBBS, HILL, DURHAM \& RICHARDSON

GENERAL ELECTRIC

\section{REGULATORY INFORMATION}

IE REGION RESPONSIBLE

IE RESIDENT INSPECTOR

LICENSING PROJ MANAGER.

RAYMOND MULLIKEN

STEVEN D. BLOON

DOCKET NUMBER.

50-285

LICENSE \& DATE ISSUANCE

DPR 040, AUGUST 09, 1973 


\section{Docket: $50-244$}

OPERATING STATUS

2. Reporting Period: DECEMBER 1994

Outage + On-Line Hrs:

744.0

3. Utility Contact: JOHN V. WALDEN (315) 524-4446 EXT. 588

4. Licensed Thermal Power (MWt):

5. Mameplate Rating (Gross MWe):

6. Design Electrical Rating (Net MWe):

7. Maximum Dependable Capacity (Gross MHe):

8. Maximum Dependable Capacity (Net MHe):

9. If Changes Occurred Above Since Last Report, Give Reasons:

10. Power Level To which Restricted, If Any (Net MHe):

11. Reasons for Restrictions, If Any:

\begin{tabular}{|c|c|c|c|}
\hline & MONTH & YEAR & CUMULATIVE \\
\hline 12. Report Period Hrs & 744.0 & $8,760.0$ & $220,008.0$ \\
\hline 13. Hours Reactor Critical & 744.0 & $7,288.8$ & $175,815.2$ \\
\hline 14. RX Reserve Shtdun Hrs & 0.0 & 0.0 & 1.687 .6 \\
\hline 15. Hrs Generator On-Line & 744.0 & $7,219.9$ & $172,972.7$ \\
\hline 16. Unit Reserve Shtdun Hrs & 0.0 & 0.0 & 8.5 \\
\hline 17. Gross Therm Ener (МนH) & $1,115,511.0$ & $10,559,224.0$ & $245,515,237.0$ \\
\hline 18. Gross Elec Ener (MUH) & $377,181.0$ & $3,550,693.0$ & $81,234,052.0$ \\
\hline 19. Net Elec Ener (MWH) & $358,923.0$ & $3,373,857.0$ & $77,109,230.0$ \\
\hline 20. Unit Service Factor & 100.0 & 82.4 & 78.6 \\
\hline 21. Unit Avail Factor & 100.0 & 82.4 & 78.6 \\
\hline 22. Unit Cap Factor (MDC Net) & 102.6 & 81.9 & 75.7 \\
\hline 23. Unit Cap Factor (DER Net) & 102.6 & 81.9 & 75.7 \\
\hline 24. Unit Forced Outage Rate & 0.0 & 5.7 & 5.6 \\
\hline 25. Forced Outage Hours & 0.0 & 434.6 & $10,353.8$ \\
\hline
\end{tabular}

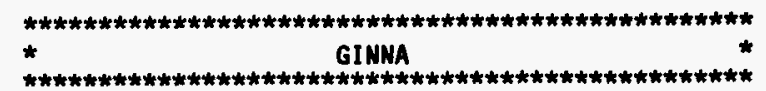

AVERAGE DAILY POWER LEVEL (Net MUe)

\begin{tabular}{ll} 
DAY & PONER \\
\hline 1 & 482 \\
\hline 2 & 482 \\
\hline 3 & 483 \\
\hline 4 & 483 \\
\hline 5 & 482 \\
\hline 6 & 482 \\
\hline 7 & 482 \\
\hline 8 & 482 \\
\hline 9 & 482 \\
\hline 10 & 483 \\
\hline 11 & 482 \\
\hline 12 & 482 \\
\hline 13 & 482 \\
\hline 14 & 483 \\
\hline 15 & 483 \\
\hline
\end{tabular}

\begin{tabular}{ll} 
DAY & PONER \\
\hline 16 & 483 \\
\hline 17 & 483 \\
\hline 18 & 483 \\
\hline 19 & 483 \\
\hline 20 & 482 \\
\hline 21 & 483 \\
\hline 22 & 483 \\
\hline 23 & 483 \\
\hline 24 & 483 \\
\hline 25 & 483 \\
\hline 26 & 483 \\
\hline 27 & 483 \\
\hline 28 & 483 \\
\hline 29 & 484 \\
\hline 30 & 484 \\
\hline 31 & 483 \\
\hline
\end{tabular}

26. Shutdowns Scheduled Over Mext Six Months (Type, Date, Duration): REFUELING/MAINTENANCE OUTAGE, MARCH 26, 1995, 36 DAYS.

27. If Currently Shutdown, Estimated Startup Date:

Notes:

CUMULATIVE REACTOR AND UNIT RESERVE SHUTDOWN HOURS ARE FROM JANUARY 1, 1975. CUMULATIVE UNIT CAPACITY FACTORS (MDC \& DER) ARE CALCULATED WITH WEIGHTED AVERAGES. LICENSEE REVISED APRIL 1994 NET ELECTRIC ENERGY FRON 88,852.0 TO 89,103.0. 


\section{TYPE}

F: Forced

S: Scheduled

\section{REASON}

A-Equipment Failure

B-Maintenance or test

D-Regulatory Restriction

E-Operator Training \&

License Examination

G-Operational Error

G-Operational Error

H-Other

\section{SYSTEM}

IEEE Standard

805-1984 and/or

MUREG-0161 Exhibit F
1-Manual

2-Manual Scran

3-Auto Scran

5-Reduced Load

9-0ther
COMPONENT

IEEE Standard

$803 A-1983$ and/or

MUREG-0161 Exhibit 
FACILITY DESCRIPTION

LOCATION

STATE ............... NEW YORK

DIST AND DIRECTION FROM

MEAREST POPULATION CTR..... $20 \mathrm{MI}$ NE OF ROCHESTER, NY

TYPE OF REACTOR

PUR

DATE INITIAL CRITICALITY...... NOVEMBER 08, 1969

DATE INITIAL ELECTRICITY $=====$ DECEMBER D2, 1969

DATE COMMERCIAL OPERATE....... JULY 01, 1970

CONDENSER COOLING METHOD...... ONCE THRU

CONDENSER COOLING WATER....... LAKE ONTARIO

ELECTRIC RELIABILITY

COUNCIL......... NORTHEASTERN POWER COORDINATION COUNCIL

\section{UTILITY \& CONTRACTOR INFORMATION}

\section{UTILITY}

LICENSEE.

CORPORATE ADDRESS.

ROCHESTER GAS \& ELECTRIC CO.

89 EAST AVENUE

ROCHESTER, NEW YORK 14649

\section{CONTRACTOR}

ARCHITECT/ENGINEER......... GILBERT ASSOCIATES

MUC STEAM SYS SUPPLIER...... MESTINGHOUSE

CONSTRUCTOR.............. BECHTEL

TURBINE SUPPLIER........... MESTINGHOUSE

\section{REGULATORY INFORMATION}

IE REGION RESPONSIBLE......... 1

IE RESIDENT INSPECTOR.......... TOM MOSLAK

LICENSING PROJ MANAGER......... ALLEN R . JOHMSON

DOCKET MUMBER.............. 50-244

LiCENSE \& DATE ISSUANCE........ DPR 018, SePtember 19, 1969 
1. Docket: $50-416$

OPERATING STATUS

2. Reporting Period: DECEMBER 1994

Outage + On-Line Hrs: $\mathbf{7 4 4 . 0}$

3. Utility Contact: S. D. LIN (601) 437-6793

4. Licensed Thermal Power (MUt):

5. Nameplate Rating (Gross MHe):

6. Design Electrical Rating (Net MHe):

7. Maximun Dependable Capacity (Gross MWe):

8. Maximum Dependable Capacity (Net Mve):

9. If Changes Occurred Above Since Last Report, Give Reasons:

10. Power Level To Which Restricted, If Any (Net MWe):

11. Reasons For Restrictions, If Any:

\begin{tabular}{|c|c|c|c|}
\hline & MONTH & YEAR & CUMULATIVE \\
\hline 12. Report Period Hrs & 744.0 & $8,760.0$ & $89,386.0$ \\
\hline 13. Hours Reactor Critical & 744.0 & $8,464.5$ & $73,151.1$ \\
\hline 14. RX Reserve Shtdwn Hrs & 0.0 & 0.0 & 0.0 \\
\hline 15. Hrs Generator On-Line & 744.0 & $8,286.5$ & $70,346.3$ \\
\hline 16. Unit Reserve Shtdwn Hrs & 0.0 & 0.0 & 0.0 \\
\hline 17. Gross Therm Ener (MUH) & $2,845,051.0$ & $31,025,924.0$ & $248,843,485.0$ \\
\hline 18. Gross Elec Ener (ММH) & $932,150.0$ & $10,013,995.0$ & $79,321,499.0$ \\
\hline 19. Net Elec Ener (MUH) & $895,417.0$ & $9,614,754.0$ & $\pi, 986,141.0$ \\
\hline 20. Unit Service Factor & 100.0 & 94.6 & 80.8 \\
\hline 21. Unit Avail Factor & 100.0 & 94.6 & 80.8 \\
\hline 22. Unit Cap Factor (MDC Net) & 105.3 & 96.0 & 78.4 \\
\hline 23. Unit Cap Factor (DER Net) & 96.3 & 87.8 & 71.2 \\
\hline 24. Unit Forced Outage Rate & 0.0 & 5.4 & 6.4 \\
\hline 25. Forced Outage Hours & 0.0 & 473.5 & $4,884.9$ \\
\hline
\end{tabular}

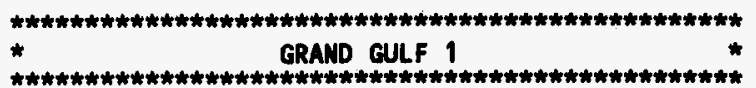

AVERAGe daily POWER LeVel (Net Mue)

\begin{tabular}{ll} 
DAY & PONER \\
\hline 1 & 1210 \\
\hline 2 & 1205 \\
\hline 3 & 1188 \\
\hline 4 & 1195 \\
\hline 5 & 1195 \\
\hline 6 & 1200 \\
\hline 7 & 1187 \\
\hline 8 & 1183 \\
\hline 9 & 1190 \\
\hline 10 & 1191 \\
\hline 11 & 1219 \\
\hline 12 & 1221 \\
\hline 13 & 1214 \\
\hline 14 & 1209 \\
\hline 15 & 1203 \\
\hline
\end{tabular}

\begin{tabular}{cl} 
DAY & POWER \\
\hline 16 & 1198 \\
\hline 17 & 1199 \\
\hline 18 & 1211 \\
\hline 19 & 1212 \\
\hline 20 & 1204 \\
\hline 21 & 1201 \\
\hline 22 & 1208 \\
\hline 23 & 1210 \\
\hline 24 & 1204 \\
\hline 25 & 1217 \\
\hline 26 & 1215 \\
\hline 27 & 1210 \\
\hline 28 & 1204 \\
\hline 29 & 1202 \\
\hline 30 & 1201 \\
\hline 31 & 1204 \\
\hline
\end{tabular}

26. Shutdowns Scheduled Over Mext Six Months (Type, Date, Duration): REFUELING OUTAGE, APRIL 15, 1995, 40 DAYS.

27. If Currently Shutdoun, Estimated Startup Date:

Notes:

CUMULATIVE VALUES FOR ITEMS $12,13,15$, AND 17-19 IMCLUDED PRE-

COMMERCIAL DATA, WHILE CUMULATIVE VALUÉS FOR ITEMS 20-25 ARE

CALCULATED SINCE COMMERCIAL OPERATION. 


No. Date Type Hours Reason Method LER Number System Component Cause and Corrective Action To Prevent Recurrence

\section{TYPE}

F: Forced

S: Scheduled

\section{REASON}

A-Equipment Failure

B-Maintenance or Test

C-Refuel ing

D-Regulatory Restriction

E-Operator Training \&

License Examination

F-Administrative

G-Operational Error

H-other

\section{METHOD \\ 1-Manual \\ 2-Manual scram \\ 3-Auto Scram \\ 4-Cont inued \\ 5-Reduced Load \\ 9-other}

\begin{tabular}{l} 
SYSTEM \\
\hline IEEE Standard \\
$805-1984$ and/or \\
MUREG-0161 Exhibit F
\end{tabular}

\section{COMPONENT}

IEEE Standard

803A-1983 and

NUREG-0161 Exhibit H 


\section{FACILITY DESCRIPTION}

\section{LOCATION}

STATE.................. MISSISSIPPI
COUNTY................ CLAIBORME

\section{DIST AND DIRECTION FROM}

MEAREST POPULATION CTR..... 25 MI S OF VICKSBURG, MS

TYPE OF REACTOR.

BUR

DATE INITIAL CRITICALITY...... AUGUST 18, 1982

DATE INITIAL ELECTRICITY...... OCTOBER 20, 1984

dATE COMmercial OPERATE....... JULY 01, 1985

CONDENSER COOLING METHOO...... CCHNDCT

CONDENSER COOLING WATER....... MISSISSIPPI RIVER

ELECTRIC RELIABILITY

COUNCIL.................... SOUTHMEST POWER POOL

\section{UTILITY \& CONTRACTOR INFORMATION}

UTILITY

LICENSEE.

CORPORATE ADDRESS

ENTERGY OPERATIONS, INC.

\section{P.O. BOX 756}

PORT GIBSON, MISSISSIPPI 39150

\section{CONTRACTOR}

ARCHITECT/ENGINEER.

BECHTEL

MUC STEAM SYS SUPPLIER...... GENERAL ELECTRIC

CONSTRUCTOR.............. BECHTEL

TURBIME SUPPLIER.......... ALLIS-CHALMERS PONER SYSTEMS INC.

\section{REGULATORY INFORMATION}

IE REGION RESPONSIBLE......... 2

IE RESIDENT INSPECTOR.......... JEFFERSON TEDRON

LICENSING PROJ MANAGER......... PAUL W. OCONMOR

DOCKET NUMBER.............. $50-416$

LICENSE \& DATE ISSUANCE........ NPF 029, AUGUST 31, 1984 
1. Docket: $50-213$

OPERATING STATUS

2. Reporting Period: DECEMBER 1994

Outage + On-Line Hrs: $\quad 744.0$

3. Utility Contact: W. M. HERWIG (203) 267-3198

4. Licensed Thermal Power (MWt):

5. Nameplate Rating (Gross MUe):

6. Design Electrical Rating (Net MHe):

7. Maximum Dependable Capacity (Gross MHe):

8. Maximum Dependable Capacity (Net MWe):

9. If Changes Occurred Above Since Last Report, Give Reasons:

10. Power Level To which Restricted, If Any (Net MWe):

11. Reasons For Restrictions, If Any:

\begin{tabular}{|c|c|c|c|}
\hline & MONTH & YEAR & CUMULATIVE \\
\hline 12. Report Period Hrs & 744.0 & $8,760.0$ & $236,688.0$ \\
\hline 13. Hours Reactor Critical & 744.0 & $6,810.2$ & $188,762.6$ \\
\hline 14. Rx Reserve Shtdun Hrs & 0.0 & 0.0 & $1,285.0$ \\
\hline 15. Hrs Generator On-Line & 711.3 & $6,756.2$ & $181,958.9$ \\
\hline 16. Unit Reserve Shtdwn Hrs & 0.0 & 0.0 & 398.0 \\
\hline 17. Gross Therm Ener (MWH) & $1,295,447.0$ & $12,182,192.0$ & $315,002,460.0$ \\
\hline 18. Gross Elec Ener (MWH) & $430,318.0$ & $3,990,621.0$ & $103,253,540.0$ \\
\hline 19. Net Elec Ener (MHH) & $410,469.4$ & $3,795,902.9$ & $98,097,176.3$ \\
\hline 20. Unit Service Factor & 95.6 & 77.1 & 76.9 \\
\hline 21. Unit Avail Factor & 95.6 & 77.1 & 77.0 \\
\hline 22. Unit Cap Factor (MDC Net) & 98.5 & 77.4 & 75.2 \\
\hline 23. Unit Cap Factor (DER Met) & 94.8 & 74.5 & 71.2 \\
\hline 24. Unit Forced Outage Rate & 4.4 & 22.9 & 6.3 \\
\hline 25. Forced Outage Hours & 32.7 & 2003.8 & $12,239.1$ \\
\hline
\end{tabular}

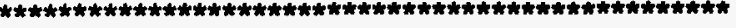 \\ ๓ HADDAM NECK}

AVERAGE DAILY PONER LEVEL (Net MWe)

\begin{tabular}{lc} 
DAY & PONER \\
\hline 1 & 579 \\
\hline 2 & 579 \\
\hline 3 & 579 \\
\hline 4 & 579 \\
\hline 5 & 578 \\
\hline 6 & 578 \\
\hline 7 & 578 \\
\hline 8 & 579 \\
\hline 9 & 579 \\
\hline 10 & 580 \\
\hline 11 & 580 \\
\hline 12 & 580 \\
\hline 13 & 580 \\
\hline 14 & 580 \\
\hline 15 & 580 \\
\hline
\end{tabular}

\begin{tabular}{cc} 
DAY & PONER \\
\hline 16 & 580 \\
\hline 17 & 580 \\
\hline 18 & 581 \\
\hline 19 & 580 \\
\hline 20 & 580 \\
\hline 21 & 579 \\
\hline 22 & 579 \\
\hline 23 & 580 \\
\hline 24 & 579 \\
\hline 25 & 578 \\
\hline 26 & 577 \\
\hline 27 & 578 \\
\hline 28 & 578 \\
\hline 29 & 578 \\
\hline 30 & 528 \\
\hline 31 & 0 \\
\hline
\end{tabular}

26. Shutdowns Scheduled Over Mext Six Months (Type, Date, Duration): REFUELING OUTAGE, JANUARY 28, 1995, 48 DAYS.

27. If Currently Shutdown, Estimated Startup Date:

Notes:

CUM VALUES FOR ITEMS 17-19 ARE FROM FIRST CRIT (JULY 24, 1967). REMAINING CUM VALUES ARE FRON COMIERCIAL OPERATION (JANUARY 1, 1968). CUM UNIT CAPACITY FACTOR (MDC NET) IS CALCULATED WITH A WEIGHTED AVERAGE. SEPTEMBER 1994 NET ELEC ENERGY REVISED. 


\begin{tabular}{cccccccccc}
\hline No. & Date & Type & Hours & Reason & Method & LER Number & system & Component & Cause and Corrective Action To Prevent Recurrence \\
\hline $94-07$ & $12 / 30 / 94$ & F & 32.7 & A & 1 & SB & V & LEAKING GASKET IN MS-NRV-2. REPLACED GASKET AND \\
RETURNED VALVE TO SERVICE.
\end{tabular}

\begin{tabular}{|c|c|}
\hline TYPE & REASON \\
\hline $\begin{array}{l}\text { F: Forced } \\
\text { S: Scheduled }\end{array}$ & $\begin{array}{l}\text { A-Equipment Failure } \\
\text { B-Maintenance or Test } \\
\text { C-Refuel ing } \\
\text { D-Regulatory Restriction } \\
\text { E-Operator Training \& } \\
\text { License Examination } \\
\text { F-Administrative } \\
\text { G-Operational Error } \\
\text { H-Other }\end{array}$ \\
\hline
\end{tabular}

\begin{tabular}{l} 
METHOD \\
\hline 1-Manual \\
2-Manual Scram \\
3-Auto Scram \\
4-Cont inued \\
5-Reduced Load \\
9-Other
\end{tabular}

\section{SYSTEM}

IEEE Standard

805-1984 and/or

MUREG-0161 Exhibit $F$

5-Reduced Load

G-Operational Error

H-Other 
*

\section{FACILITY DESCRIPTION}

\section{LOCATION}

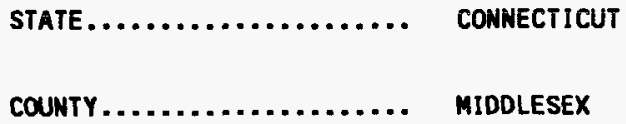

\section{DIST AND DIRECTION FROM}

\section{MEAPEST POPULATIOH CTR.}

TYPE OF REACTOR

DATE INITIAL CRITICALITY......

DATE INITIAL ELECTRICITY......

DATE COMMERCIAL OPERATE.

CONDENSER COOLING METHOD.

CONDENSER COOLING WATER.

ELECTRIC RELIABILITY

COUNCIL...............

\section{UTILITY \& CONTRACTOR INFORMATION}

\section{UTILITY}

LICENSEE.

CORPORATE ADDRESS.

CONTRACTOR

ARCHITECT/ENGINEER.......... STONE \& HEBSTER

NUC STEAM SYS SUPPLIER...... WESTINGHOUSE

CONSTRUCTOR. . . . . . . . . . . .

TURBINE SUPPLIER........... WESTINGHOUSE

\section{REGULATORY INFORMATION}

IE REGION RESPONSIBLE........ 1

IE RESIDENT INSPECTOR.

WILLIAM J. RAYMOND

LICENSING PROJ MANAGER.

ALAN B. WANG

COORDINATION COUNCIL
DOCKET NUMBER

$50-213$

LICENSE \& DATE ISSUANCE
DPR 061, DECEMBER 27, 1974 
1. Docket: $50-400$

2. Reporting Period: DECEMBER 1994

Outage + On-Line Hrs:

744.0

3. Utility Contact: SHEILA ROGERS (919) 362-2573

4. Licensed Thermal Power (MUt):

5. Nameplate Rating (Gross MWe):

6. Design Electrical Rating (Net MHe):

7. Maximum Dependable Capacity (Gross MWe):

8. Maximum Dependable Capacity (Net MHe):

\begin{tabular}{c}
\hline 2775 \\
\hline 951 \\
\hline 900 \\
\hline 920 \\
\hline 860 \\
\hline
\end{tabular}

9. If Changes Occurred Above Since Last Report, Give Reasons:

10. Power Level To Which Restricted, If Any (Net MWe):

11. Reasons For Restrictions, If Any:

\begin{tabular}{|c|c|c|c|}
\hline & MONTH & YEAR & CUMULATIVE \\
\hline 12. Report Period Hrs & 744.0 & $8,760.0$ & $67,225.0$ \\
\hline 13. Hours Reactor Critical & 744.0 & $7,247.6$ & $55,549.9$ \\
\hline 14. Rx Reserve Shtdwn Hrs & 0.0 & 0.0 & 0.0 \\
\hline 15. Hrs Generator On-Line & 744.0 & $7,196.4$ & $54,973.9$ \\
\hline 16. Unit Reserve Shtdwn Hrs & 0.0 & 0.0 & 0.0 \\
\hline 17. Gross Therm Ener (MUH) & $2,015,342.6$ & $19,556,637.0$ & $146,815,283.8$ \\
\hline 18. Gross Elec Ener (МHH) & 671.951 .0 & $6,489,305.0$ & $48,923,526.0$ \\
\hline 19. Net Elec Ener (MHH) & $630,066.0$ & $6,057,236.0$ & $45,590,637.0$ \\
\hline 20. Unit Service Factor & 100.0 & 82.2 & 81.8 \\
\hline 21. Unit Avail Factor & 100.0 & 82.2 & 81.8 \\
\hline 22. Unit Cap Factor (MDC Net) & 98.5 & 80.4 & 78.9 \\
\hline 23. Unit Cap Factor (DER Net) & 94.1 & 76.8 & 75.4 \\
\hline 24. Unit Forced Outage Rate & 0.0 & 0.0 & 2.9 \\
\hline 25. Forced Outage Hours & 0.0 & 0.0 & $1,660.0$ \\
\hline
\end{tabular}

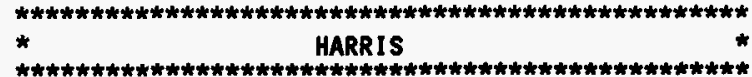

AVERAGE DAILY PONER LEVEL (Net MHe)

\begin{tabular}{ll} 
DAY & POWER \\
1 & 879 \\
\hline 2 & 877 \\
\hline 3 & 871 \\
\hline 4 & 816 \\
\hline 5 & 859 \\
\hline 6 & 867 \\
\hline 7 & 867 \\
\hline 8 & 874 \\
\hline 9 & 879 \\
\hline 10 & 759 \\
\hline 11 & 379 \\
\hline 12 & 723 \\
\hline 13 & 874 \\
\hline 14 & 872 \\
\hline 15 & 869 \\
\hline
\end{tabular}

\begin{tabular}{ll} 
DAY & PONER \\
16 & 872 \\
\hline 17 & 873 \\
\hline 18 & 875 \\
\hline 19 & 878 \\
\hline 20 & 878 \\
\hline 21 & 876 \\
\hline 22 & 873 \\
\hline 23 & 873 \\
\hline 24 & 872 \\
\hline 25 & 876 \\
\hline 26 & 876 \\
\hline 27 & 875 \\
\hline 28 & 872 \\
\hline 29 & 873 \\
\hline 30 & 874 \\
\hline 31 & 873 \\
\hline
\end{tabular}

26. Shutdowns Scheduled Over Next Six Months (Type, Date, Duration):

27. If Currently Shutdown, Estimated Startup Date:

Notes: 


\begin{tabular}{|c|c|c|c|c|c|c|c|c|c|}
\hline Mo. & Date & Type & Hours & Reason & Method & LER Nunber & Systen & Conponent & Cause and Corrective Action To Prevent Recurrence \\
\hline 94-012 & $12 / 10 / 94$ & $\mathbf{F}$ & 0.0 & A & 5 & & Нн & HTEXCH & $\begin{array}{l}\text { "A" CONDENSATE BOOSTER PUIP RENONED FROM SERVICE. } \\
\text { EXCESSIVE WATER IN OIL DUE TO A FAILED TUBE IN } \\
\text { LUBE OIL HEAT EXCHANGER. }\end{array}$ \\
\hline
\end{tabular}

\begin{tabular}{|c|c|}
\hline TYPE & REASON \\
\hline $\begin{array}{l}\text { F: Forced } \\
\text { S: Scheduled }\end{array}$ & $\begin{array}{l}\text { A-Equipment Failure } \\
\text { B-Maintenance or Test } \\
\text { C-Refuel ing } \\
\text { D-Regulatory Restriction } \\
\text { E-Operator Training \& } \\
\text { License Examination } \\
\text { F-Administrative } \\
\text { G-Operational Error } \\
\text { H-Other }\end{array}$ \\
\hline
\end{tabular}

\begin{tabular}{l} 
METHCo \\
\hline 1-Manual \\
2-Manual Scram \\
3-Auto Scram \\
4-Cont inued \\
5-Reduced Load \\
9-0ther
\end{tabular}

\section{SYSTEM}

IEEE Standard

and/or

NUREG-0161 Exhibit $F$
COMPONENT

IEEE Standard
$8034-1983$ and/o

MUREG-0161 Exhibit 


\section{FACILITY DESCRIPTION}

\section{LOCATION}

STATE................. NORTH CAROLINA
COUNTY............... WAKE \& CHAPHAM cos.

\section{DIST AMD DIRECTION FROM}

NEAREST POPULATION CTR..... 20 MI SW OF RALEIGH, NC

TYPE OF REACTOR.

PUR

DATE INITIAL CRITICALITY

DATE INITIAL ELECTRICITY

DATE COMmERCIAL OPERATE.

CONDENSER COOLING METHOO.

CONDENSER COOLING WATER.

ELECTRIC RELIABILITY

COUNCIL.................. SOUTHEASTERN ELECTRIC

RELIABILITY COUNCIL

MaY 02, 1987

NDCT

MAKEUP RESERVOIR

\section{UTILITY \& CONTRACTOR INFORMATION}

\section{UTILITY}

LICENSEE. .

CORPORATE AODRESS.

CAROLIMA POWER \& LIGHT CO.

P.O. BOX 1551

RALEIGH, NORTH CAROLIMA 27602

\section{CONTRACTOR}

ARCHI TECT/ENGINEER.

NUC STEAM SYS SUPPLIER

CONSTRUCTOR.

TURBINE SUPPLIER 
1. Docket: $50-321$

OPERATING STATUS

2. Reporting Period: DECEMBER 1994

Outage + On-Line Hrs: $\quad 744.0$

3. Utility Contact: T. W. TIDWELL (912) 367-7781 EXT. 2878

4. Licensed Thermal Power (MWt):

5. Nameplate Rating (Gross MWe):

6. Design Electrical Rating (Net MWe):

7. Maximum Dependable Capacity (Gross MWe):

8. Maximum Dependable Capacity (Net MHe):

9. If Changes Occurred Above Since Last Report, Give Reasons:

10. Power Level To Which Restricted, If Any (Net MHe):

11. Reasons For Restrictions, If Any:

\begin{tabular}{c}
\hline 2436 \\
\hline 850 \\
\hline 776 \\
\hline 774 \\
\hline 741
\end{tabular}

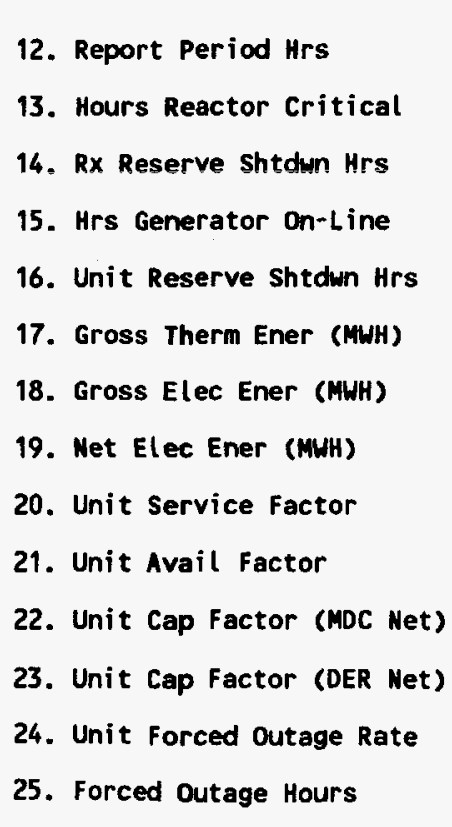

$\begin{array}{r}\text { MONTH } \\ 744.0 \\ \hline 744.0 \\ \hline 0.0 \\ \hline 744.0 \\ \hline 0.0 \\ \hline 1,807,154.0 \\ \hline 594,536.0 \\ \hline 570,074.0 \\ \hline 100.0 \\ \hline 100.0 \\ \hline 103.4 \\ \hline 98.7 \\ \hline 0.0 \\ \hline 0.0 \\ \hline\end{array}$

\begin{tabular}{|c|c|c|c|}
\hline \multirow{3}{*}{$\begin{array}{l}\text { YEAR } \\
8,760.0\end{array}$} & \multirow{3}{*}{$\begin{array}{l}\text { CUMULATIVE } \\
166,559.0\end{array}$} & 9 & 762 \\
\hline & & 10 & 762 \\
\hline & & 11 & 769 \\
\hline $7,638.1$ & $125,641.6$ & & \\
\hline 0.0 & 0.0 & 16 & I \\
\hline & & 13 & 768 \\
\hline & & 14 & 770 \\
\hline 0.0 & 0.0 & 15 & 769 \\
\hline
\end{tabular}

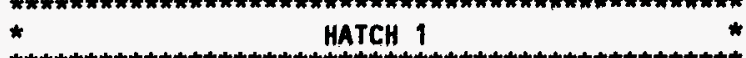

AVERAGE DAILY POWER LEVEL (Net MHe)

$5,767,986.0 \quad 87,112,316.0$

$\overline{5,507,212.0} \quad \overline{82,897,706.0}$

$86.1-72.3$

86.1
84.8

81.0
1.5

11.3

$15,378.5$

\begin{tabular}{ll} 
DAY & PONER \\
\hline 1 & 769 \\
\hline 2 & 769 \\
\hline 3 & 747 \\
\hline 4 & 758 \\
\hline 5 & 761 \\
\hline 6 & 739 \\
\hline 7 & 763 \\
\hline 8 & 762 \\
\hline 9 & 762 \\
\hline 10 & 762 \\
\hline 11 & 769 \\
\hline 12 & 771 \\
\hline 13 & 768 \\
\hline 14 & 770 \\
\hline 15 & 769 \\
\hline
\end{tabular}

\begin{tabular}{cc} 
DAY & POWER \\
\hline 16 & 767 \\
\hline 17 & 770 \\
\hline 18 & 769 \\
\hline 19 & 773 \\
\hline 20 & 770 \\
\hline 21 & 770 \\
\hline 22 & 770 \\
\hline 23 & 769 \\
\hline 24 & 768 \\
\hline 25 & 772 \\
\hline 26 & 771 \\
\hline 27 & 772 \\
\hline 28 & 772 \\
\hline 29 & 769 \\
\hline 30 & 763 \\
\hline 31 & 769 \\
\hline
\end{tabular}

26. Shutdowns Scheduled Over Next Six Months (Type, Date, Duration):

27. If Currently Shutdown, Estimated Startup Date:

Notes:

CUMULATIVE UNIT CAPACITY FACTORS (MDC \& DER) ARE CALCULATED WITH WEIGHTED AVERAGES. 


No. Date Type Hours Reason Method LER Number System Component Cause and Corrective Action To Prevent Recurrence

\section{TYPE}

F: Forced

S: Scheduled

\begin{tabular}{l} 
REASON \\
\hline A-Equipment Failure \\
B-Maintenance or Test \\
C-Refuel ing \\
D-Regulatory Restriction \\
E-Operator Training \& \\
License Examination \\
F-Adninistrative \\
G-Operational Error \\
H-Other
\end{tabular}

F-Administrative

H-other
SYSTEM

IEEE Standard

805-1984 and/or

NUREG-0161 Exhibit F
COMPONENT

IEEE Standard

803A-1983 and/or

NUREG-0161 Exhibit H

2-Manual Scram

3-Auto Scram

5-Reduced

9-0ther 


\section{FACILITY DESCRIPTION}

\section{LOCATION}

STATE..$\ldots \ldots \ldots \ldots \ldots \ldots \ldots$.
COUNTY.................. APPLING

\section{DIST AND DIRECTION FRON} MEAREST POPULATION CTR....

TYPE OF REACTOR.

11 MI N OF BAXLEY, GA

DATE INITIAL CRITICALITY...... SEPTEMBER 12, 1974

DATE INITIAL ELECTRICITY...... NOVEMBER 11, 1974

DATE COMMERCIAL OPERATE....... DECEMBER 31, 1975

CONDENSER COOLIMG METHOO...... COOLING TONER

CONDENSER COOLIMG WATER....... ALTAMAHA RIVER

ELECTRIC RELIABILITY

COUNCIL .................. SOUTHEASTERN ELECTRIC

RELIABILITY COUNCIL

\section{UTILITY \& CONTRACTOR IMFORMATION}

\section{UTILITY}

LICENSEE............... GeORgIA PONER CO.

CORPORATE ADDRESS......... P.O. BOX 1295

BIRMINGHAM, ALABAMA 35201

\section{CONTRACTOR}

ARCHI TECT/ENGINEER

BECHTEL

NUC STEAM SYS SUPPLIER......

GENERAL ELECTRIC

CONSTRUCTOR

GEORGIA PONER CO.

TURBINE SUPPL.IER

GENERAL ELECTRIC

REGULATORY INFORMATION

IE REGION RESPONSIBLE......... 2

IE RESIDENT INSPECTOR......... BOBBY HOLBROOK

LICENSING PROJ MANAGER.......... KAHTAN N. JABBOUR

DOCKET MUMBER............... 50-321

LICENSE \& DATE ISSUANCE......... DPR 057, OCTOBER 13, 1974 
1. Docket: $50-366$

OPERATING STATUS

2. Reporting Period: DECEMBER 1994

Outage + On-Line Hrs: $\quad 744.0$

3. Utility Contact: T. W. TIDWELL (912) 367-7781 EXT. 2878

4. Licensed Thermal Power (mWt):

5. Nameplate Rating (Gross MWe):

6. Design Electrical Rating (Net MWe):

7. Maximun Dependable Capacity (Gross MHe):

8. Maximum Dependable Capacity (Net MHe):

9. If Changes Occurred Above Since Last Report, Give Reasons:

10. Power Level To Which Restricted, If Any (Net MWe):

11. Reasons for Restrictions, If Any:

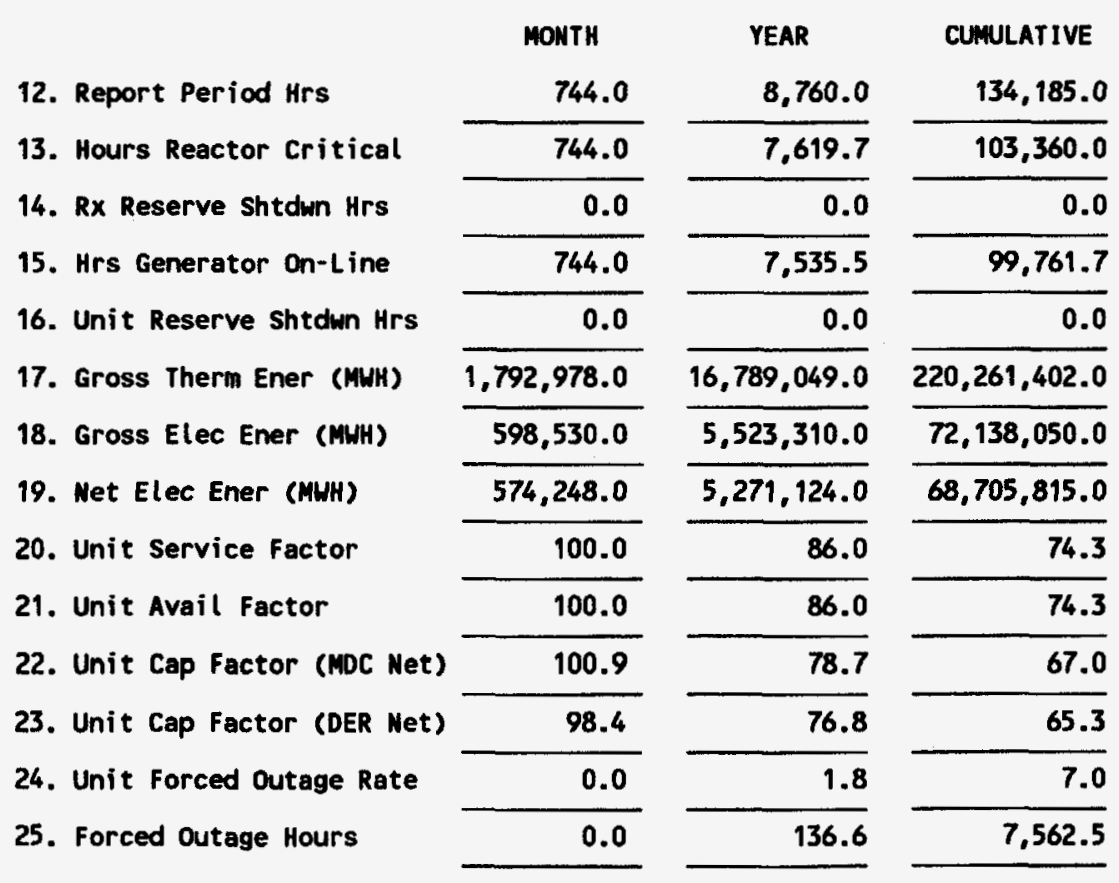

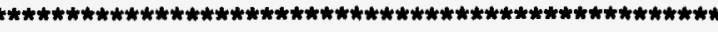

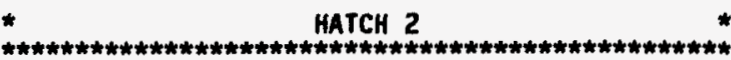

AVERAGE DAILY POWER LEVEL (Net MWe)

\begin{tabular}{ll} 
DAY & POWER \\
\hline 1 & 781 \\
\hline 2 & 779 \\
\hline 3 & 772 \\
\hline 4 & 767 \\
\hline 5 & 772 \\
\hline 6 & 773 \\
\hline 7 & 779 \\
\hline 8 & 776 \\
\hline 9 & 778 \\
\hline 10 & 605 \\
\hline 11 & 722 \\
\hline 12 & 781 \\
\hline 13 & 782 \\
\hline 14 & 780 \\
\hline 15 & 779 \\
\hline
\end{tabular}

\begin{tabular}{ll} 
DAY & POWER \\
\hline 16 & 781 \\
\hline 17 & 774 \\
\hline 18 & 777 \\
\hline 19 & 783 \\
\hline 20 & 783 \\
\hline 21 & 782 \\
\hline 22 & 782 \\
\hline 23 & 780 \\
\hline 24 & 783 \\
\hline 25 & 783 \\
\hline 26 & 781 \\
\hline 27 & 783 \\
\hline 28 & 784 \\
\hline 29 & 782 \\
\hline 30 & 781 \\
\hline 31 & 780 \\
\hline
\end{tabular}

26. Shutdowns Scheduled Over Next Six Months (Type, Date, Duration):

27. If Currently Shutdown, Estimated Startup Date:

Notes:

CUNULATIVE UNIT CAPACITY FACTORS (MDC \& DER) ARE CALCULATED WITH WEIGHTED AVERAGES. 


$\begin{array}{cccccc}\text { No. } & \text { Date } & \text { Type } & \text { Hours } & \text { Reason } & \text { Met } \\ 94-011 & 12 / 10 / 94 & \text { S } & 0.0 & \text { F } & 5\end{array}$

LER Number System

Component

Cause and Corrective Action To Prevent Recurrence

REDUCED LOAD TO PERFORM A CONTROL ROD SEQUENCE EXCHANGE AND CONTROL ROD SCRAM TIME TESTING. WHILE AT REDUCED PONER, PERFORMEd TURBIME CONTROL VALVE AND TURBINE BYPASS VALVE TESTING, AND CONTROL ROD DRIVE EXERCISES FOR SELECTED ROD DRIVES.

\begin{tabular}{|c|c|}
\hline TYPE & REASON \\
\hline $\begin{array}{l}\text { F: Forced } \\
\text { S: Scheduled }\end{array}$ & $\begin{array}{l}\text { A-Equipment Failure } \\
\text { B-Maintenance or Test } \\
\text { C-Refuel ing } \\
\text { D-Regulatory Restriction } \\
\text { E-Operator Training \& } \\
\text { License Examination } \\
\text { F-Administrative } \\
\text { G-Operational Error } \\
\text { H-Other }\end{array}$ \\
\hline
\end{tabular}

\begin{tabular}{l} 
METHCO \\
\hline 1-Manual \\
2-Manual Scram \\
3-Auto Scram \\
4-Cont inued \\
5-Reduced Load \\
9-0ther
\end{tabular}

SYSTEM

IEEE Standard

805-1984 and/or

NUREG-0161 Exhibit $F$
COMPONENT

IEEE Standard

803A-1983 and/or

NUREG-0161 Exhibit H 


\section{FACILITY DESCRIPTION}

\section{LOCATION}

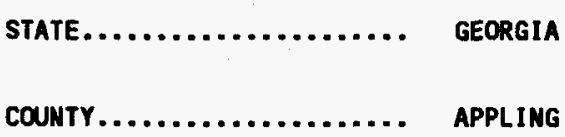

DIST AND DIRECTION FRON NEAREST POPULATION CTR.

TYPE OF REACTOR.

11 MI N OF BAXLEY, GA

DATE INITIAL CRITICALITY...... JULY 04, 1978

DATE INITIAL ELECTRICITY...... SEPTEMBER 22, 1978

DATE COMMERCIAL OPERATE........ SEPTEMBER 05, 1979

CONDENSER COOLING METHOD...... COOLING TONER

CONDENSER COOLING WATER....... ALTAMAHA RIVER

ELECTRIC RELIABILITY

COUNCIL.................. SOUTHEASTERM ELECTRIC RELIABILITY COUNCIL

\section{UTILITY \& CONTRACTOR INFORMATION}

UTILITY

LICENSEE.

GEORGIA PONER CO.

CORPORATE ADDRESS........... P.O. BOX 1295 BirminghaM, ALABAMA 35201

\section{CONTRACTOR}

ARCHITECT/ENGINEER.......... BECHTEL

MUC STEAM SYS SUPPLIER...... GENERAL ELECTRIC

CONSTRUCTOR............... GEORGIA PONER CO.

TURBINE SUPPLIER........... GENERAL ELECTRIC

REGULATORY INFORMATION

IE REGION RESPONSIBLE......... 2

IE RESIDENT INSPECTOR.......... BOBBY HOLBROOK

LICENSING PROJ MANAGER......... KAHTAN N. JABBOUR

DOCKET MUMBER.............. 50-366

LICENSE \& DATE ISSUANCE....... NPF 005, JUNE 13, 1978 
1. Docket: $50-354$

OPERATING STATUS

2. Reporting Period: DECEMBER 1994

Outage + On-Line Hrs: 744.0

3. Utility Contact: D. U. LYONS (609) 339-3517

4. Licensed Thermal Power (MUt):

5. Nameplate Rating (Gross MHe):

6. Design Electrical Rating (Net MUe):

7. Maximum Dependable Capacity (Gross MWe):

8. Maximum Dependable Capacity (Net MWe):

9. If Changes Occurred Above Since Last Report, Give Reasons:

10. Power Level To Which Restricted, If Any (Net MWe):

11. Reasons For Restrictions, If Any:

\begin{tabular}{|c|c|c|c|}
\hline & MONTH & YEAR & CUMULATIVE \\
\hline 12. Report Period Hrs & 744.0 & $8,760.0$ & $70,416.0$ \\
\hline 13. Hours Reactor Critical & 744.0 & $7,112.9$ & $59,935.9$ \\
\hline 14. Rx Reserve Shtdun Mrs & 0.0 & 0.0 & $0=0$ \\
\hline 15. Hrs Generator On-Line & 744.0 & $6,970.9$ & $59,005.3$ \\
\hline 16. Unit Reserve Shtdun Hrs & 0.0 & 0.0 & 0.0 \\
\hline 17. Gross Therm Ener (MUH) & $2,430,315.0$ & $22,450,973.0$ & $188,414,343.0$ \\
\hline 18. Gross Elec Ener (MWH) & $819,216.0$ & $7,463,712.0$ & $62,427,225.0$ \\
\hline 19. Net Elec Ener (MUH) & $786,182.0$ & $7,125,636.0$ & $59,652,880.0$ \\
\hline 20. Unit Service Factor & 100.0 & 79.6 & 83.8 \\
\hline 21. Unit Avail Factor & 100.0 & 79.6 & 83.8 \\
\hline 22. Unit Cap Factor (MDC Net) & 102.5 & 78.9 & 82.2 \\
\hline 23. Unit Cap Factor (DER Net) & 99.0 & 76.2 & 79.4 \\
\hline 24. Unit Forced Outage Rate & 0.0 & 6.9 & 4.9 \\
\hline 25. Forced Outage Hours & 0.0 & 514.5 & $3,033.4$ \\
\hline
\end{tabular}

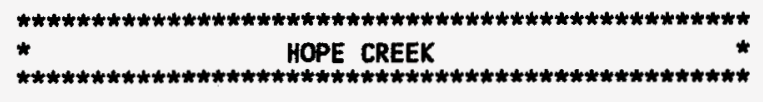

AVERAGE DAILY PONER LEVEL (Het MHe)

\begin{tabular}{ll} 
DAY & PONER \\
\hline 1 & 1067 \\
\hline 2 & 1066 \\
\hline 3 & 1063 \\
\hline 4 & 1058 \\
\hline 5 & 1052 \\
\hline 6 & 1056 \\
\hline 7 & 1053 \\
\hline 8 & 1064 \\
\hline 9 & 1069 \\
\hline 10 & 1045 \\
\hline 11 & 949 \\
\hline 12 & 1071 \\
\hline 13 & 1065 \\
\hline 14 & 1062 \\
\hline 15 & 1063 \\
\hline
\end{tabular}

\begin{tabular}{cc} 
DAY & PONER \\
\hline 16 & 1059 \\
\hline 17 & 1062 \\
\hline 18 & 1054 \\
\hline 19 & 1063 \\
\hline 20 & 1063 \\
\hline 21 & 1060 \\
\hline 22 & 1061 \\
\hline 23 & 1057 \\
\hline 24 & 1050 \\
\hline 25 & 1057 \\
\hline 26 & 1059 \\
\hline 27 & 1060 \\
\hline 28 & 1053 \\
\hline 29 & 1069 \\
\hline 30 & 1066 \\
\hline 31 & 1063 \\
\hline
\end{tabular}

26. Shutdowns Scheduled Over Next Six Months (Type, Date, Duration):

27. If Currently Shutdown, Estimated Startup Date:

Notes: 
A N D

POWER

REDUCTIONS

\begin{tabular}{l} 
SYSTEM \\
\hline IEEE Standard \\
$805-1984$ and/or \\
NUREG-0161 Exhibit
\end{tabular}

\section{METHOD}

1-Manual

2-Manual Scram

3-Auto Scram

4-Cont inued

5-Reduced Load

9-0ther

\section{IEEE Standard}

803A-1983 and/or

NUREG-0161 Exhibit H 


\section{FACILITY DESCRIPTION}

\section{LOCATION}

STATE................. NEW JERSEY

COUNTY................ SALEM

\section{DIST AND DIRECTION FROM}

NEAREST POPULATION CTR......

TYPE OF REACTOR.

8 MI SE OF WILMINGTON, DE

DATE INITIAL CRITICALITY......

DATE INITIAL ELECTRICITY......

DATE COMmERCIAL OPERATE.

CONDENSER COOLING METHOD......

CONDENSER COOLING WATER

ELECTRIC RELIABILITY

COUNCIL................... MID-ATLANTIC AREA COUNCIL

JUNE 28,1986

AUGUST 01, 1986

DECEMBER 20, 1986

NDCT

DELAMARE RIVER

\section{UTILITY \& CONTRACTOR INFORMATION}

UTILITY

LICEMSEE................ PUblic SERVICE EleCtric \& gAS CO.

CORPORATE ADDRESS......... 80 PARK PLACE

NEWARK, NEY JERSEY 07101

\section{CONTRACTOR}

ARCHI TECT/ENGINEER. .

NUC STEAM SYS SUPPLIER.

BECHTEL

CONSTRUCTOR.

GENERAL ELECTRIC

TURBINE SUPPLIER

BECHTEL

GEMERAL ELECTRIC

REGULATORY INFORMATION

IE REGION RESPONSIBLE.........

IE RESIDENT INSPECTOR.

LICENSING PROJ MANAGER

DOCKET MUMBER.

LICENSE \& DATE ISSUANCE
1

ROBERT SUMMERS

JAMES C. STONE

$50-354$

MPF 057, JULY 25, 1986 


\section{Docket: $50-247$}

OPERATING STATUS

2. Reporting Period: DECEMBER 1994

outage + On-Line Hrs: $\mathbf{7 4 4 . 0}$

3. Utility Contact: A. REED (914) 526-5155

4. Licensed Thermal Power (MUt):

5. Nameplate Rating (Gross MWe):

6. Design Electrical Rating (Net MHe):

7. Maximum Dependable Capacity (Gross MWe):

8. Maximum Dependable Capacity (Het MHe):

9. If Changes Occurred Above since Last Report, Give Reasons:

10. Power Level To Which Restricted, If Any (Net MWe):

11. Reasons For Restrictions, If Any:

\begin{tabular}{|c|c|c|c|}
\hline 12. Report Period Hrs & $\begin{array}{l}\text { MONTH } \\
744.0\end{array}$ & $\begin{array}{l}\text { YEAR } \\
8,760.0\end{array}$ & $\begin{array}{l}\text { CUMULATIVE } \\
179,737.0\end{array}$ \\
\hline 13. Hours Reactor Critical & 744.0 & $8,760.0$ & $128,370.9$ \\
\hline 14. Rx Reserve Shtdin Hrs & 0.0 & 0.0 & $4,118.5$ \\
\hline 15. Hrs Generator on-Line & 744.0 & $8,760.0$ & $125,309.1$ \\
\hline 16. Unit Reserve Shtdin Hrs & 0.0 & 0.0 & 0.0 \\
\hline 17. Gross Therm Ener (MUH) & $2,033,758.0$ & $24,711,827.0$ & $345,894,751.0$ \\
\hline 18. Gross Elec Ener (MWH) & $660,546.0$ & $7,916,978.0$ & $106,309,561.0$ \\
\hline 19. Net Elec Ener (MHH) & $636,797.0$ & $7,634,604.0$ & $101,784,834.0$ \\
\hline 20. Unit Service Factor & 100.0 & 100.0 & 69.7 \\
\hline 21. Unit Avail Factor & 100.0 & 100.0 & 69.7 \\
\hline 22. Unit Cap Factor (MDC Net) & 90.0 & 92.8 & 64.7 \\
\hline 23. Unit Cap Factor (DER Net) & 86.8 & 88.4 & 63.0 \\
\hline 24. Unit Forced Outage Rate & 0.0 & 0.0 & 6.4 \\
\hline 25. Forced Outage Hours & 0.0 & 0.0 & $8,613.0$ \\
\hline
\end{tabular}

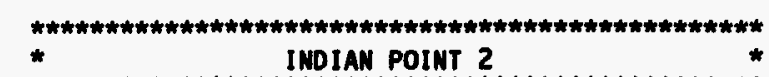

IINDIAN POINT 2 IN

AVERAGE DAILY PONER LEVEL (Net MUe)

\begin{tabular}{lc} 
DAY & PONER \\
\hline 1 & 855 \\
\hline 2 & 858 \\
\hline 3 & 861 \\
\hline 4 & 859 \\
\hline 5 & 856 \\
\hline 6 & 855 \\
\hline 7 & 858 \\
\hline 8 & 859 \\
\hline 9 & 856 \\
\hline 10 & 856 \\
\hline 11 & 854 \\
\hline 12 & 854 \\
\hline 13 & 855 \\
\hline 14 & 855 \\
\hline 15 & 856 \\
\hline
\end{tabular}

\begin{tabular}{|l|l}
\hline DAY & POWER \\
\hline 16 & 856 \\
\hline 17 & 855 \\
\hline 18 & 854 \\
\hline 19 & 855 \\
\hline 20 & 857 \\
\hline 21 & 858 \\
\hline 22 & 854 \\
\hline 23 & 854 \\
\hline 24 & 855 \\
\hline 25 & 854 \\
\hline 26 & 855 \\
\hline 27 & 857 \\
\hline 28 & 854 \\
\hline 29 & 855 \\
\hline 30 & 855 \\
\hline 31 & 857 \\
\hline
\end{tabular}

26. Shutdowns Scheduled Over Next Six Months (Type, Date, Duration): REFUELING OUTAGE, FEBRUARY 4, 1995, 93 DAYS.

27. If Currently Shutdown, Estimated Startup Date:

Notes:

YEAR-TO-DATE AND CUMULATIVE UNIT CAPACITY FACTORS (MDC \& DER) ARE CALCULATED HITH WEIGHTED AVERAGES. 


\section{TYPE}

F: Forced

S: Scheduled

\section{REASON}

A-Equipment Failure

B-Maintenance or Test

C-Refuel ing

D-Regulatory Restriction

E-Operator Training \&

icense Examination

F-Administrative

G-Operational Error

H-Other

\section{METHOD}

1-Manua 2-Manual Scram 3-Auto Scram

4-Continued

5 -Reduced Load

9-0ther

\section{SYSTEM}

IEEE Standard 805-1984 and/or NUREG-0161 Exhibit $F$
COMPONENT

IEEE Standard 803A-1983 and/or NUREG-0161 Exhibit H 


\section{FACILITY DESCRIPTION}

\section{LOCATION}

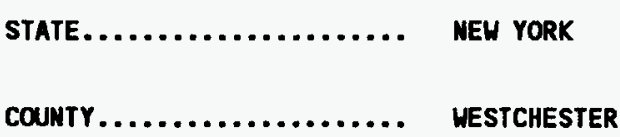

\section{DIST AND DIRECTION FROM} MEAREST POPULATION CTR.

TYPE OF REACTOR.

DATE INITIAL CRITICALITY...... MAY 22, 1973

DATE INITIAL ELECTRICITY...... JUNE 26, 1973

DATE COMmERCIAL OPERATE....... AUGUST 01, 1974

CONDENSER COOLING METHOD...... ONCE THRU

CONDENSER COOLING WATER....... HUDSON RIVER

\section{ELECTRIC RELIABILITY}

COUNCIL.................. MORTHEASTERH POWER

COORDINATION COUNCIL

\section{UTILITY \& CONTRACTOR INFORMATION}

UTILITY

LICENSEE. CONSOLIDATED EDISON CO. OF M.Y.

CORPORATE ADDRESS

4 IRVING PLACE

NEW YORK, NEW YORK 10017

\section{CONTRACTOR}

ARCHI TECT/ENGINEER .

UNITED ENG. \& CONSTRUCTORS

NUC STEAM SYS SUPPLIER...... WESTINGHOUSE

CONSTRUCTOR

WEST INGHOUSE DEVELOPNENT CORP

TURBINE SUPPLIER.

VEST INGHOUSE

\section{REGULATORY INFORMATION}

IE REGION RESPONSIBLE......... 1

IE RESIDENT INSPECTOR........ GORDON K. HUNEGS

LICENSING PROJ MANAGER.......... FRANCIS J. MILLIAMS

DOCKET MUMBER............... 50-247

LICENSE \& DATE ISSUANCE........ DPR 026, SEPTEMBER 28, 1973 


\section{Docket: $50-286$}

OPERATING STATUS

2. Reporting Period: DECEMBER 1994

Outage + On-Line Hrs: 744.0

3. Utility Contact: T. ORLANDO (914) 736-8340

4. Licensed Thermal Power (MHT):

5. Nameplate Rating (Gross MWe):

6. Design Electrical Rating (Net MHe):

7. Maximum Dependable Capacity (Gross MWe):

8. Maximum Dependable Capacity (Net Mue):

9. If Changes Occurred Above Since Last Report, Give Reasons:

10. Power Level To Which Restricted, If Any (Net Mue):

11. Reasons for Restrictions, If Any:

\begin{tabular}{|c|c|c|c|}
\hline & MONTH & YEAR & CUMULATIVE \\
\hline 12. Report Period Hrs & 744.0 & $8,760.0$ & $160,753.0$ \\
\hline 13. Hours Reactor Critical & 0.0 & 0.0 & $91,890.3$ \\
\hline 14. Rx Reserve Shtodun Hrs & 0.0 & $0 . \hat{0}$ & 0.0 \\
\hline 15. Hrs Generator On-Line & 0.0 & 0.0 & 89.462 .2 \\
\hline 16. Unit Reserve Shtdwn Hrs & 0.0 & 0.0 & 0.0 \\
\hline 17. Gross Therm Ener (MUH) & 0.0 & 0.0 & $254,069,702.0$ \\
\hline 18. Gross Elec Ener (MWH) & 0.0 & 0.0 & $79,388,605.0$ \\
\hline 19. Net Elec Ener (MUH) & 0.0 & 0.0 & $76,357,136.0$ \\
\hline 20. Unit Service Factor & 0.0 & 0.0 & $\begin{array}{r}55.7 \\
\end{array}$ \\
\hline 21. Unit Avail Factor & 0.0 & $\overline{0.0}$ & 55.7 \\
\hline 22. Unit Cap Factor (MDC Net) & 0.0 & 0.0 & 50.4 \\
\hline 23. Unit Cap Factor (DER Net) & 0.0 & 0.0 & 49.2 \\
\hline 24. Unit Forced Outage Rate & 100.0 & 100.0 & 25.9 \\
\hline 25. Forced Outage Hours & 744.0 & 8760.0 & $31,345.8$ \\
\hline
\end{tabular}

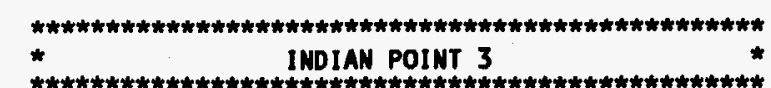

* INDIAN POINT 3 ,

AVERAGE DAILY PONER LEVEL (Net MWe)

\begin{tabular}{lr} 
DAY & PONER \\
\hline 1 & 0 \\
\hline 2 & 0 \\
\hline 4 & 0 \\
\hline 5 & 0 \\
\hline 6 & 0 \\
\hline 7 & 0 \\
\hline 8 & 0 \\
\hline 9 & 0 \\
\hline 10 & 0 \\
\hline 11 & 0 \\
\hline 12 & 0 \\
\hline 13 & 0 \\
\hline 14 & 0 \\
\hline 15 & 0 \\
\hline
\end{tabular}

\begin{tabular}{ll} 
DAY & PONER \\
\hline 16 & 0 \\
\hline 17 & 0 \\
\hline 18 & 0 \\
\hline 19 & 0 \\
\hline 20 & 0 \\
\hline 21 & 0 \\
\hline 22 & 0 \\
\hline 23 & 0 \\
\hline 24 & 0 \\
\hline 25 & 0 \\
\hline 26 & 0 \\
\hline 27 & 0 \\
\hline 28 & 0 \\
\hline 29 & 0 \\
\hline 30 & 0 \\
\hline 31 & 0 \\
\hline
\end{tabular}

26. Shutdowns Scheduled Over Next Six Months (Type, Date, Duration):

27. If Currently Shutdown, Estimated Startup Date: 02/17/95

Notes:

CUMULATIVE UNIT CAPACITY FACTOR (MDC RET) IS CALCULATED MITH A WEIGHTED AVERAGE. 
A N D

POWER

REDUCTIONS

\begin{tabular}{cccccccccc}
\hline No. & Date & Type & Hours & Reason & Method & LER Number & System & Component & Cause and Corrective Action To Prevent Recurrence \\
\hline 1 & $02 / 27 / 93$ & $F$ & 744.0 & B & 4 & 93005 & IE & IMSTRU & THE UNIT WAS REMOVED FROM SERVICE IN ORDER TO \\
PERFORM TESTING ON THE PLANT'S AHSAC SYSTEM.
\end{tabular}

\begin{tabular}{l} 
TYPE \\
\hline F: Forced \\
S: Scheduled
\end{tabular}

\begin{tabular}{l} 
REASON \\
\hline A-Equipment Failure \\
B-Maintenance or Test \\
C-Refueling \\
D-Regulatory Restriction \\
E-Operator Training \& \\
License Examination \\
F-Administrative \\
G-Operational Error \\
H-Other
\end{tabular}

\section{METHOD}

1-Manual

2-Manual Scram

3-Auto Scram

5-Reduced Load

9-Other

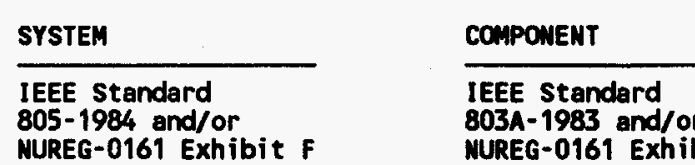

NUREG-0161 Exhibit F NUREG-0161 Exhibit

G-Operational Error 


\section{FACILITY DESCRIPTION}

\section{LOCATION}

STATE.

COUNTY

DIST AND DIRECTION FROM NEAREST POPULATION CTR....

TYPE OF REACTOR

DATE INITIAL CRITICALITY.

DATE INITIAL ELECTRICITY

DATE COMERCIAL OPERATE

CONDENSER COOLING METHOO

CONDENSER COOLING HATER.

ELECTRIC RELIABILITY

COUNCIL.................... MORTHEASTERN POWER

COORDINATION COUNCIL

\section{UTILITY \& CONTRACTOR INFORMATION}

UT ILITY

LICENSEE. NEW YORK POWER AUTHORITY

CORPORATE ADDRESS

10 COLUMBUS CIRCLE NEW YORK, NEW YORK 10019

\section{CONTRACTOR}

ARCHI TECT/ENGINEER.

MUC STEAM SYS SUPPLIER...... WESTINGHOUSE

CONSTRUCTOR

WESTINGHOUSE DEVELOPMENT CORP

TURBINE SUPPLIER.

WEST INGHOUSE

REGULATORY INFORMATION

IE REGION RESPONSIBLE........ 1

IE RESIDENT INSPECTOR......... DAVID LEW

LICENSING PROJ MANAGER......... NICOLA F. CONICELLA

DOCKET NUMBER............ 50-286

LICENSE \& DATE ISSUANCE........ DPR 064, APRIL 05, 1976 
1. Docket: $50-305$

OPERATING STATUS

2. Reporting Period: DECEMBER 1994

Outage + On-Line Hrs: $\quad 744.0$

3. Utility Contact: M. L. ANDERSON (414) 388-2560 EXT. 2453

4. Licensed Thermal Power (MUt):

5. Nameplate Rating (Gross Mue):

6. Design Electrical Rating (Net MWe):

7. Maximum Dependable Capacity (Gross MWe):

8. Maximun Dependable Capacity (Net MWe):

9. If Changes Occurred Above Since Last Report, Give Reasons:

10. Power Level To thich Restricted, If Any (Net MWe):

11. Reasons for Restrictions, If Any:
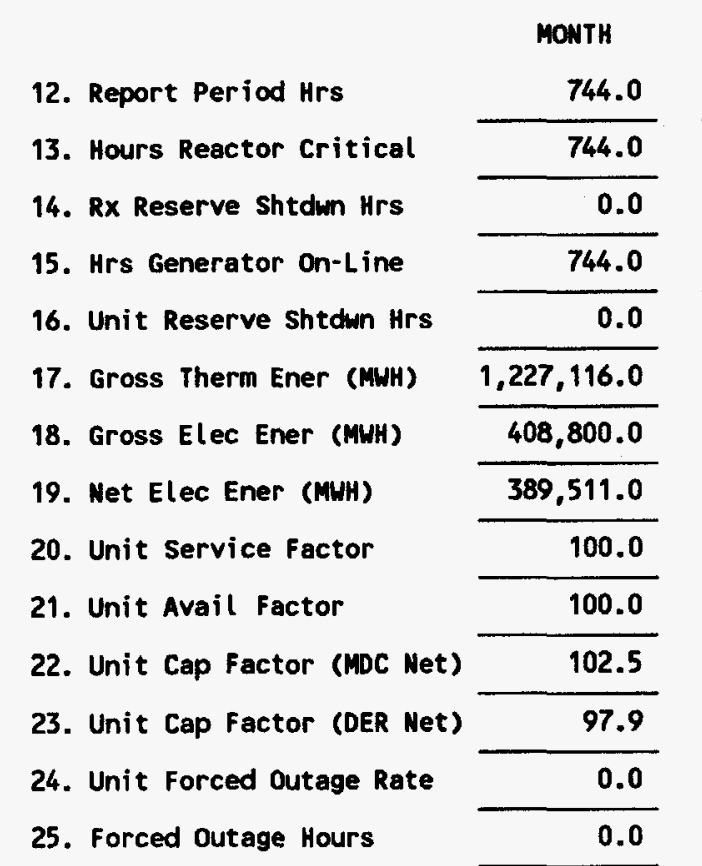

\begin{tabular}{c}
1650 \\
\hline 560 \\
\hline 535 \\
\hline 537 \\
\hline 511
\end{tabular}

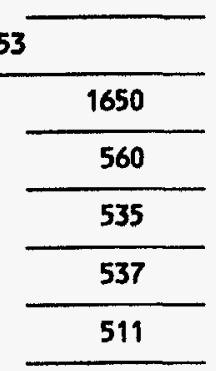




\section{TYPE}

F: Forced

S: Scheduled

\begin{tabular}{l} 
REASON \\
\hline A-Equipment Failure \\
B-Maintenance or Test \\
C-Refuel ing \\
D-Regulatory Restriction \\
E-Operator Training \& \\
License Examination \\
F-Administrative \\
G-Operat ional Error \\
H-Other
\end{tabular}

METHOD

1-Manual

2-Manual Scram

3-Auto Scram

4-Cont inued

5-Reduced Load

9-other

$\begin{array}{ll}\text { SYSTEM } & \text { COMPONENT } \\ \text { IEEE Standard } & \text { IEEE Standard } \\ 805-1984 \text { and/or } & \text { 803A-1983 and/or } \\ \text { MUREG-0161 Exhibit F } & \text { NUREG-0161 Exhibit }\end{array}$

MUREG-0161 Exhibit F NUREG-0161 Exhibit

G-Operational Error 


\section{FACILITY DESCRIPTION}

LOCATION

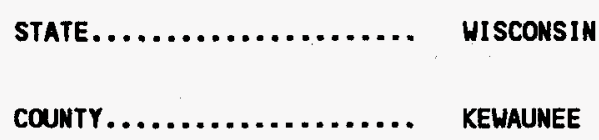

\section{DIST AND DIRECTION FROM}

NEAREST POPULATION CTR..... 27 MI E OF GREEN BAY, HI

TYPE OF REACTOR

PWR

DATE INITIAL CRITICALITY...... MARCH 07, 1974

DATE INITIAL ELECTRICITY...... APRIL 08, 1974

DATE COMmERCial OPERATE....... JUNE 16, 1974

CONDENSER COOLING METHOO...... ONCE THRU

CONDENSER COOLING MATER....... LAKE MICHIGAN

ELECTRIC RELIABILITY

COUNCIL.................. MID-AMERICA INTERPOOL NETWORK

\section{UTILITY \& CONTRACTOR INFORMATION}

UTILITY

LICENSEE.

WISCONSIN PUBLIC SERVICE CORP.

CORPORATE ADDRESS

P.O. BOX 19002

GREEN BAY, HISCONSIN 54307

\section{CONTRACTOR}

ARCHITECT/ENGINEER.

NUC STEAM SYS SUPPLIER...... MESTINGHOUSE

CONSTRUCTOR

PUBLIC SERVICES AND gAS COMPANY

TURBINE SUPPLIER.

WEST INGHOUSE

REGULATORY INFORMATION

IE REGION RESPONSIBLE......... 3

IE RESIDENT INSPECTOR......... JAMES HELLER

LICENSING PROJ MANAGER......... R. J. LAUFER

DOCKET NUMBER.............. 50.305

LICENSE \& DATE ISSUANCE........ DPR 043, DECEMBER 21, 1973 
1. Docket: $50-373$

OPERATING STATUS

2. Reporting Period: DECEMBER 1994

Outage + On-Line Hrs: 744.0

3. Utility Contact: M. J. CIALKOWSKI (815) 357-6761 EXT. 2427

4. Licensed Thermal Power (MUt):

5. Nameplate Rating (Gross MHe):

6. Design Electrical Rating (Net MWe):

7. Maximum Dependable Capacity (Gross MWe):

8. Maximum Dependable Capacity (Net MWe):

9. If Changes Occurred Above Since Last Report, Give Reasons:

10. Power Level To Which Restricted, If Any (Net MWe):

11. Reasons For Restrictions, If Any:

\begin{tabular}{|c|c|c|c|}
\hline & MONTH & YEAR & CUMULAT IVE \\
\hline 12. Report Period Hrs & 744.0 & $8,760.0$ & $96,432.0$ \\
\hline 13. Hours Reactor Critical & 570.3 & $5,313.0$ & $66,640.1$ \\
\hline 14. Rx Reserve Shtdun Hrs & 0.0 & 0.0 & $1,641.2$ \\
\hline 15. Hrs Generator On-Line & 538.4 & $5,097.5$ & $65,110.9$ \\
\hline 16. Unit Reserve Shtdwn Hrs & 0.0 & 0.0 & 1.0 \\
\hline 17. Gross Therm Ener (MUH) & $1,603,693.0$ & $15,361,188.0$ & $192,686,825.4$ \\
\hline 18. Gross Elec Ener (MHH) & $525,061.0$ & $5,136,520.0$ & $64,390,891.0$ \\
\hline 19. Net Elec Ener (MUH) & $505,918.0$ & $4,920,390.0$ & $61,769,144.0$ \\
\hline 20. Unit Service Factor & 72.4 & 58.2 & 67.5 \\
\hline 21. Unit Avail Factor & 72.4 & 58.2 & 67.5 \\
\hline 22. Unit Cap Factor (MDC Net) & 65.6 & 54.2 & 61.8 \\
\hline 23. Unit Cap Factor (DER Net) & 63.1 & 52.1 & 59.4 \\
\hline 24. Unit Forced Outage Rate & 27.6 & 16.6 & 8.3 \\
\hline 25. Forced Outage Hours & 205.6 & 1014.7 & $5,868.7$ \\
\hline
\end{tabular}

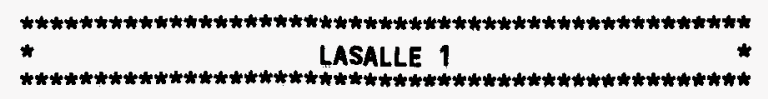

AVERAGE DAILY PONER LEVEL (Net MWe)

\begin{tabular}{ll} 
DAY & PONER \\
\hline 1 & 1099 \\
\hline 2 & 1101 \\
\hline 3 & 1099 \\
\hline 4 & 1098 \\
\hline 5 & 1097 \\
\hline 6 & 1096 \\
\hline 7 & 1097 \\
\hline 8 & 1099 \\
\hline 9 & 1098 \\
\hline 10 & 1102 \\
\hline 11 & 1079 \\
\hline 12 & 892 \\
\hline 13 & -12 \\
\hline 14 & -12 \\
\hline 15 & -12 \\
\hline
\end{tabular}

DAY POWER

\begin{tabular}{ll}
16 & -12 \\
\hline 17 & -12
\end{tabular}

$\longdiv { 1 8 } 9 1$

$\begin{array}{r}79 \\ \hline\end{array}$

\begin{tabular}{ll}
\hline 20 & -12 \\
\hline 21 & -12
\end{tabular}

\begin{tabular}{ll}
21 & -12 \\
\hline 22 & -12
\end{tabular}

23269

$24 \quad 856$

$25 \quad 838$

\begin{tabular}{ll}
\hline 26 & 857 \\
\hline 27 & 922
\end{tabular}

\begin{tabular}{ll}
\hline 28 & 1087 \\
\hline 29 & 1095 \\
\hline 30 & 1085 \\
\hline 31 & 1042 \\
\hline
\end{tabular}

26. Shutdowns Scheduled Over Next six Months (Type, Date, Duration):

27. If Currently Shutdown, Estimated Startup Date:

Notes: 


\begin{tabular}{|c|c|c|c|c|c|c|c|c|c|}
\hline No. & Date & Type & Hours & Reason & Method & LER Number & System & Component & Cause and Corrective Action To Prevent Recurrence \\
\hline 8 & $12 / 12 / 94$ & $\mathbf{F}$ & 126.8 & A & 3 & 94015 & & & $\begin{array}{l}\text { REACTOR SCRAM DUE TO GROUP I ISOLATION DURING A } \\
\text { MAIN STEAM HIGH FLOW SWITCH SURVEILLANCE. }\end{array}$ \\
\hline 9 & $12 / 19 / 94$ & $\mathbf{F}$ & 78.8 & A & 1 & & & & $\begin{array}{l}\text { MANUAL REACTOR SHUTDOWN DUE TO PROBLEMS MITH THE } \\
\text { ROO POSITION INDICATION SYSTEM. }\end{array}$ \\
\hline
\end{tabular}

\begin{tabular}{|c|c|}
\hline TYPE & REASON \\
\hline $\begin{array}{l}\text { F: Forced } \\
\text { S: Scheduled }\end{array}$ & $\begin{array}{l}\text { A-Equipment Failure } \\
\text { B-Maintenance or Test } \\
\text { C-Refuel ing } \\
\text { D-Regulatory Restriction } \\
\text { E-Operator Training \& } \\
\text { License Examination } \\
\text { F-Administrative } \\
\text { G-Operational Error } \\
\text { H-Other }\end{array}$ \\
\hline
\end{tabular}

\begin{tabular}{l} 
METHOD \\
\hline 1-Manual \\
2-Manual Scram \\
3-Auto Scram \\
4-Cont inued \\
5-Reduced Load \\
9-Other
\end{tabular}

\begin{tabular}{|c|c|}
\hline SYSTEM & COMPONENT \\
\hline $\begin{array}{l}\text { IEEE Standard } \\
805-1984 \text { and/or } \\
\text { NUREG-0161 Exhibit F }\end{array}$ & $\begin{array}{l}\text { IEEE Standard } \\
803 A-1983 \text { and/or } \\
\text { MUREG-0161 Exhibit }\end{array}$ \\
\hline
\end{tabular}

F-Administrative

H-Other 


\section{FACILITY DESCRIPIION}

LOCATION

STATE.

COUNTY

\section{DIST AND DIRECTION FROM}

MEAREST POPULATION CTR..... 11 MI SE OF OTTAWA, IL

TYPE OF REACTOR

DATE INITIAL CRITICALITY...... JUNE 21, 1982

DATE INITIAL ELECTRICITY...... SEPTEMBER 04, 1982

Date COMmERcial OPERATE....... January 01, 1984

CONDENSER COOLING METHOD...... POND

CONDENSER COOLING WATER....... RESERVOIR

ELECTRIC RELIABILITY

COUNCIL................... MID-AMERICA INTERPOOL NETWORK

\section{UTILITY \& CONTRACTOR INFORMATION}

\section{UTILITY}

LICENSEE.

CORPORATE ADDRESS.

COMHONWEALTH EDISON CO.

1400 OPUS PL., OPUS WEST III

SUITE 300
DOWNER'S GROVE, ILLINOIS 60515

CONTRACTOR

ARCHITECT/ENGINEER .

SARGENT \& LUNDY

NUC STEAM SYS SUPPLIER...... GENERAL ELECTRIC

CONSTRUCTOR............... COMMONWEALTH EDISON

TURBINE SUPPLIER.......... GENERAL ELECTRIC

\section{REGULATORY INFORMATION}

IE REGION RESPONSIBLE......... 3

IE RESIDENT INSPECTOR......... PHILIP BROCKMAN

LICENSING PROJ MANAGER......... HILLIAM D. RECKLEY

DOCKET NUMBER.

$50-373$

LICENSE \& DATE ISSUANCE.

NPF 011, AUGUST 13, 1982 
1. Docket: $50-374$

OPERATING STATUS

2. Reporting Period: DECEMBER 1994

Outage + On-Line Hrs: $\mathbf{7 4 4 . 0}$

3. Utility Contact: M. J. CIALKOWSKI (815) 357-6761 EXT. 2427

4. Licensed Thermal Power (MUt):

5. Nameplate Rating (Gross MUe):

6. Design Electrical Rating (Net MWe):

7. Maximum Dependable Capacity (Gross MWe):

8. Maximum Dependable Capacity (Het MHe):

\begin{tabular}{l}
\hline 3323 \\
\hline 1146 \\
\hline 1078 \\
\hline 1146 \\
\hline 1036 \\
\hline
\end{tabular}

9. If Changes Occurred Above Since Last Report, Give Reasons:

10. Power Level To Which Restricted, If Any (Net MHe):

11. Reasons For Restrictions, If Any:

\begin{tabular}{|c|c|c|c|}
\hline & MONTH & YEAR & CUMULAT IVE \\
\hline 12. Report Period Hrs & 744.0 & $8,760.0$ & $89,424.0$ \\
\hline 13. Hours Reactor Critical & 744.0 & $8,282.4$ & $65,208.1$ \\
\hline 14. Rx Reserve Shtdwn Hrs & 0.0 & 0.0 & $1,716.9$ \\
\hline 15. Hrs Generator On-Line & 744.0 & $8,103.2$ & $63,985.9$ \\
\hline 16. Unit Reserve Shtdun Hrs & 0.0 & 0.0 & 0.0 \\
\hline 17. Gross Therm Ener (MUH) & $2,417,108.0$ & $25,651,475.0$ & $193,751,928.2$ \\
\hline 18. Gross Elec Ener (MUH) & $826,694.0$ & $8,703,929.0$ & $64,688,569.0$ \\
\hline 19. Net Elec Ener (MUH) & $801,611.0$ & $8,428,871.0$ & $62,184,762.0$ \\
\hline 20. Unit Service Factor & 100.0 & 92.5 & 71.6 \\
\hline 21. Unit Avail Factor & 100.0 & 92.5 & 71.6 \\
\hline 22. Unit Cap Factor (MDC Met) & 104.0 & 92.9 & 67.1 \\
\hline 23. Unit Cap Factor (DER Net) & 99.9 & 89.3 & 64.5 \\
\hline 24. Unit Forced Outage Rate & 0.0 & 4.4 & 10.6 \\
\hline 25. Forced Outage Hours & 0.0 & 374.5 & $7,576.2$ \\
\hline
\end{tabular}

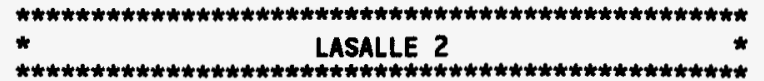

AVERAge dAILY POWER LeVel (Net MHe)

\begin{tabular}{ll} 
DAY & POWER \\
\hline 1 & 1032 \\
\hline 2 & 1103 \\
\hline 3 & 1102 \\
\hline 4 & 1102 \\
\hline 5 & 1098 \\
\hline 6 & 1097 \\
\hline 7 & 1098 \\
\hline 8 & 1099 \\
\hline 9 & 1100 \\
\hline 10 & 1100 \\
\hline 11 & 1096 \\
\hline 12 & 1097 \\
\hline 13 & 1096 \\
\hline 14 & 1095 \\
\hline 15 & 1086 \\
\hline
\end{tabular}

\begin{tabular}{cc}
\hline DAY & PONER \\
\hline 16 & 1100 \\
\hline 17 & 1100 \\
\hline 18 & 1095 \\
\hline 19 & 1094 \\
\hline 20 & 1095 \\
\hline 21 & 1102 \\
\hline 22 & 1098 \\
\hline 23 & 1098 \\
\hline 24 & 1017 \\
\hline 25 & 849 \\
\hline 26 & 1026 \\
\hline 27 & 1094 \\
\hline 28 & 1101 \\
\hline 29 & 1103 \\
\hline 30 & 985 \\
\hline 31 & 1042 \\
\hline
\end{tabular}

26. Shutdowns Scheduled Over Hext Six Months (Type, Date, Duration): REFUELING OUTAGE, February 18, 1995, 13 weEks.

27. If Currently Shutdown, Estimated Startup Date:

Notes: 


\begin{tabular}{|c|c|}
\hline TYPE & REASON \\
\hline $\begin{array}{l}\text { F: Forced } \\
\text { s: Scheduled }\end{array}$ & $\begin{array}{l}\text { A-Equipment Failure } \\
\text { B-Maintenance or Test } \\
\text { C-Refuel ing } \\
\text { D-Regulatory Restriction } \\
\text { E-Operator Iraining \& } \\
\text { License Examination } \\
\text { F-Administrative } \\
\text { G-Operational Error } \\
\text { H-Other }\end{array}$ \\
\hline
\end{tabular}

\section{METHOD}

1-Manual

2-Manual Scran

3-Auto Scram

4-Cont inued

5-Reduced Load

9-other

\begin{tabular}{l} 
SYSTEM \\
\hline IEEE Standard \\
$805-1984$ and/or \\
NUREG-0161 Exhibit F
\end{tabular}

NUREG-0161 Exhibit $F$
COMPONENT

IEEE Standard

$803 A-1983$ and/or

NUREG-0161 Exhibit H

G-Administrative

H-Othe 
FACILITY DESCRIPTION

LOCATION

STATE.

countr.

ILLIMOIS

LA SALLe

DIST AND DIRECTION FROM

NEAREST POPULAT

TYPE OF REACTOR

BWR

DATE INITIAL CRITICALITY...... MARCH 10,1984

DATE INITIAL ELECTRICITY...... APRIL 20, 1984

DATE COMmERCIAL OPERATE........ JUNE 19, 1984

CONDENSER COOLING METHOD...... POND

CONDENSER COOLING WATER....... RESERVOIR

ELECTRIC RELIABILITY

COUHCIL.................... MID-AMERICA INTERPOOL NETWORK

\section{UTILITY \& CONTRACTOR INFORMATION}

UTILITY

LICENSEE.

CORPORATE ADDRESS.

CONTRACTOR

ARCHITECT/ENGINEER

MUC STEAM SYS SUPPLIER.

CONSTRUCTOR.

TURBINE SUPPLIER

COMMONWEALTH EDISON CO.

1400 OPUS PL., OPUS VEST III

SUITE 300

DONNER'S GROVE, ILLINOIS 60515

\section{REGULATORY INFORMATION}

IE REGION RESPONSIBLE...........

IE RESIDENT INSPECTOR........... PHILIP BROCKMAN

LICENSING PROJ MANAGER......... HILLIAM D. RECKLEY

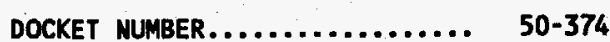

LICENSE \& DATE ISSUANCE........ NPF 018, MARCH 23, 1984 
1. Docket: $50-352$

OPERATING STATUS

2. Reporting Period: DECEMBER 1994

Outage + On-Line Hrs: $\quad 744.0$

3. Utility Contact: STEVEN J. KELLEY (610) 718-3763

4. Licensed Thermal Power (MUt):

5. Nameplate Rating (Gross MHe):

6. Design Electrical Rating (Wet MWe):

7. Maximm Dependable Capacity (Gross MWe):

8. Maximum Dependable Capacity (Het MHe):

9. If Changes Occurred Above Since Last Report, Give Reasons:

10. Power Level To Which Restricted, If Any (Net MWe):

11. Reasons for Restrictions, If Any:

\begin{tabular}{|c|c|c|c|}
\hline Report Period Hrs & $\begin{array}{l}\text { MONTH } \\
744.0\end{array}$ & $\begin{array}{l}\text { YEAR } \\
8,760.0\end{array}$ & $\begin{array}{r}\text { CUMULATIVE } \\
78,144.0\end{array}$ \\
\hline Hours Reactor Critical & 744.0 & $7,909.2$ & $64,108.3$ \\
\hline Rx Reserve Shtdwn Hrs & 0.0 & 0.0 & 0.0 \\
\hline Hrs Generator On-Line & 744.0 & $7,840.7$ & $63,025.5$ \\
\hline Unit Reserve Shtdwn Hrs & 0.0 & 0.0 & 0.0 \\
\hline Gross Therm Ener (MUH) & $2,447,604.0$ & $24,865,778.0$ & $194,316,539.0$ \\
\hline Gross Elec Ener (MHH) & $813,920.0$ & $8,142,240.0$ & $63,184,280.0$ \\
\hline Net Elec Ener (MHH) & $787,009.0$ & $7,858,016.0$ & $60,644,006.0$ \\
\hline Unit Service Factor & 100.0 & 89.5 & 80.7 \\
\hline Unit Avail Factor & 100.0 & 89.5 & 80.7 \\
\hline Unit Cap Factor (MDC Net) & 100.3 & 85.0 & 73.6 \\
\hline Unit Cap Factor (DER Net) & 100.3 & 85.0 & 73.6 \\
\hline Unit Forced Outage Rate & 0.0 & 0.9 & 4.3 \\
\hline Forced Outage Hours & 0.0 & 68.9 & $2,814.8$ \\
\hline
\end{tabular}

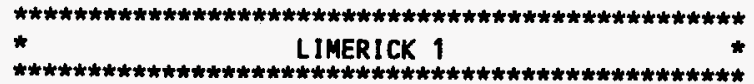

AVERAGE DAILY POWER LEVEL (Net MWe)

\begin{tabular}{ll} 
DAY & PONER \\
\hline 1 & 1057 \\
\hline 2 & 1055 \\
\hline 3 & 1056 \\
\hline 4 & 1052 \\
\hline 5 & 1046 \\
\hline 6 & 1047 \\
\hline 7 & 1065 \\
\hline 8 & 1044 \\
\hline 9 & 1060 \\
\hline 10 & 1064 \\
\hline 11 & 1054 \\
\hline 12 & 1060 \\
\hline 13 & 1063 \\
\hline 14 & 1037 \\
\hline 15 & 1061 \\
\hline
\end{tabular}

\begin{tabular}{ll} 
DAY & POWER \\
\hline 16 & 1060 \\
\hline 17 & 1060 \\
\hline 18 & 1060 \\
\hline 19 & 1060 \\
\hline 20 & 1056 \\
\hline 21 & 1061 \\
\hline 22 & 1057 \\
\hline 23 & 1057 \\
\hline 24 & 1056 \\
\hline 25 & 1060 \\
\hline 26 & 1064 \\
\hline 27 & 1064 \\
\hline 28 & 1060 \\
\hline 29 & 1060 \\
\hline 30 & 1069 \\
\hline 31 & 1064 \\
\hline
\end{tabular}

26. Shutdowns Scheduled Over Next Six Months (Type, Date, Duration):

27. If Currently Shutdown, Estimated Startup Date:

Notes: 


No. Date Type Hours Reason Method LER Mumber System Component Cause and Corrective Action To Prevent Recurrence

\begin{tabular}{|c|c|c|c|c|}
\hline TYPE & REASON & METHOD & SYSTEM & COMPONENT \\
\hline $\begin{array}{l}\text { F: Forced } \\
\text { S: Scheduled }\end{array}$ & $\begin{array}{l}\text { A-Equipment Failure } \\
\text { B-Maintenance or Test } \\
\text { C-Refuel ing } \\
\text { D-Regulatory Restriction } \\
\text { E-Operator Training \& } \\
\text { License Examination } \\
\text { F-Administrative } \\
\text { G-Operat ional Error } \\
\text { H-Other }\end{array}$ & $\begin{array}{l}\text { 1-Manual } \\
\text { 2-Manual Scram } \\
\text { 3-Auto Scram } \\
\text { 4-Cont inued } \\
\text { 5-Reduced Load } \\
\text { 9-0ther }\end{array}$ & $\begin{array}{l}\text { IEEE Standard } \\
\text { 805-1984 and/or } \\
\text { NUREG-0161 Exh ibit F }\end{array}$ & $\begin{array}{l}\text { IEEE Standard } \\
803 \mathrm{~A}-1983 \text { and/or } \\
\text { NUREG-0161 Exhibit H }\end{array}$ \\
\hline
\end{tabular}




\section{FACILITY DESCRIPTION}

LOCATION

STATE.$\ldots \ldots \ldots \ldots \ldots \ldots \ldots$ PENWSYLVANIA
COUNTY.................. MONTGOMERY

DIST AND DIRECTION FROM

MEAREST POPULATION CTR..... 21 MI NW OF PHILADELPHIA, PA

TYPE OF REACTOR

BWR

DATE INITIAL CRITICALITY...... DECEMBER 22, 1984

DATE INITIAL ELECTRICITY...... APRIL 13, 1985

DATE COMMERCIAL OPERATE....... FEBRUARY 01, 1986

CONDENSER COOLING METHOD....... COOLING TOWER

CONDENSER COOLING HATER....... SCHUYLKILL RIVER

ELECTRIC RELIABILITY

COUHCIL.................... MID-ATLANTIC AREA COUHCIL

\section{UTILITY \& CONTRACTOR INFORMATION}

UTILITY

LICENSEE

CORPORATE ADDRESS

\section{CONTRACTOR}

ARCHI TECT/ENGINEER

NUC STEAM SYS SUPPLIER.

BECHTEL

CONSTRUCTOR.

GENERAL ELECTRIC

TURBINE SUPPLIER

BECHTEL

GEMERAL ELECTRIC

REGULATORY INFORMATION

IE REGION RESPONSIBLE.........

IE RESIDENT INSPECTOR......... NEIL PERRY

LICENSING PROJ MANAGER........ FRANK RINALDI

DOCKET NUMBER.............. 50-352

LICENSE \& DATE ISSUANCE........ MPF 039, AUGUST 08, 1985 
1. Docket: $50-353$

OPERATING STATUS

2. Reporting Period: DECEMBER 1994

Outage + On-Line Hrs: $\quad 744.0$

3. Utility Contact: STEVEN J. KELLEY (610) 718-3763

4. Licensed Thermal Power (MWt):

5. Nameplate Rating (Gross mWe):

6. Design Electrical Rating (Net MWe):

7. Maximum Dependable Capacity (Gross MWe):

8. Maximum Dependable Capacity (Net MWe):

9. If Changes Occurred Above Since Last Report, Give Reasons:

10. Power Level To Which Restricted, If Any (Net MWe):

11. Reasons For Restrictions, If Any:

\begin{tabular}{c}
$\frac{3293}{1138}$ \\
\hline$\frac{1055}{1092}$ \\
\hline 1055 \\
\hline
\end{tabular}

12. Report Period Hrs

13. Hours Reactor Critical

14. Rx Reserve Shtdwn Hrs

15. Hrs Generator On-Line

16. Unit Reserve Shtdun Hrs

17. Gross Therm Ener (MUH)

18. Gross Elec Ener (MHH)

19. Net Elec Ener (MUH)

20. Unit Service Factor

21. Unit Avail factor

22. Unit Cap Factor (MDC Net)

23. Unit Cap Factor (DER Net)

24. Unit Forced Outage Rate

25. Forced Outage Hours

\section{MONTH}

$$
\text { YEAR }
$$

CUMULATIVE

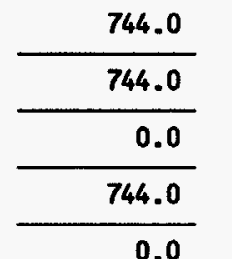

$\overline{1,672,906.0}$

$527,934.0$

$502,562.0$

100.0

100.0

$\frac{64.0}{64.0}$

\begin{tabular}{c}
0.0 \\
\hline 0.0
\end{tabular}

\begin{tabular}{|c|c|c|c|}
\hline 8.760 .0 & 43.656 .0 & 10 & 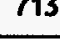 \\
\hline 8700 & & 11 & 700 \\
\hline ( 525 & & 12 & 702 \\
\hline 0.0 & 0.0 & 13 & 702 \\
\hline $8,658.2$ & $38,604.9$ & 14 & 695 \\
\hline 0.0 & 0.0 & 15 & 685 \\
\hline
\end{tabular}
Notes:
L

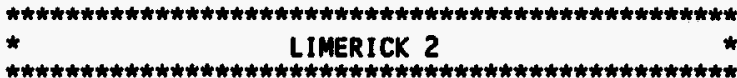

AVERAGe DAILY POWER LeVEL (Net MHe)

\begin{tabular}{ll} 
DAY & POWER \\
\hline 1 & 752 \\
\hline 2 & 747 \\
\hline 3 & 741 \\
\hline 4 & 725 \\
\hline 5 & 620 \\
\hline 6 & 624 \\
\hline 7 & 651 \\
\hline 8 & 702 \\
\hline 9 & 720 \\
\hline 10 & 713 \\
\hline 11 & 700 \\
\hline 12 & 702 \\
\hline 13 & 702 \\
\hline 14 & 695 \\
\hline 15 & 685 \\
\hline
\end{tabular}

\begin{tabular}{cc} 
DAY & PONER \\
\hline 16 & 685 \\
\hline 17 & 677 \\
\hline 18 & 677 \\
\hline 19 & 674 \\
\hline 20 & 666 \\
\hline 21 & 665 \\
\hline 22 & 658 \\
\hline 23 & 655 \\
\hline 24 & 651 \\
\hline 25 & 646 \\
\hline 26 & 646 \\
\hline 27 & 642 \\
\hline 28 & 638 \\
\hline 29 & 618 \\
\hline 30 & 638 \\
\hline 31 & 626 \\
\hline
\end{tabular}

26. Shutdowns Scheduled Over Next Six Months (Type, Date, Duration): REFUELING OUTAGE, JANUARY 28, 1995.

27. If Currently Shutdown, Estimated Startup Date: 


\begin{tabular}{cccccccccc}
\hline No. & Date & Type & Hours & Reason & Method & LER Number & System & Component & Cause and Corrective Action To Prevent Recurrence \\
\hline 28 & $12 / 04 / 94$ & $S$ & 0.0 & B & 5 & RB & CRDRVE & REACTOR PONER HAS REDUCED FOR H.C.U. MAIMTENANCE.
\end{tabular}

\begin{tabular}{|c|c|}
\hline TYPE & REASON \\
\hline $\begin{array}{l}\text { F: Forced } \\
\text { S: Scheduled }\end{array}$ & $\begin{array}{l}\text { A-Equipment Failure } \\
\text { B-Maintenance or Test } \\
\text { C-Refuel ing } \\
\text { D-Regulatory Restriction } \\
\text { E-Operator Training \& } \\
\text { License Examination } \\
\text { F-Adninistrative } \\
\text { G-Operational Error } \\
\text { H-Other }\end{array}$ \\
\hline
\end{tabular}

\begin{tabular}{l} 
METHOD \\
\hline 1-Manual \\
2-Manual Scram \\
3-Auto Scram \\
4-Cont inued \\
5-Reduced Load \\
9-0ther
\end{tabular}

\section{SYSTEM}

IEEE Standard

805-1984 and/or

NUREG-0161 Exhibit $F$

\section{COMPONENT}

IEEE Standard

NUREG-0161 Exhibit H 


\section{FACILITY DESCRIPTION}

\section{LOCATION}

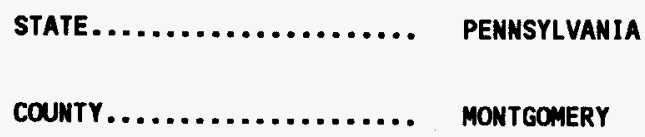

\section{DIST AND DIRECTION FROM} MEAREST POPULATION CTR.

TYPE OF REACTOR

DATE INITIAL CRITICALITY

DATE INITIAL ELECTRICITY

DATE COMMERCIAL OPERATE.

CONDENSER COOLING METHOD.

CONDENSER COOLING WATER.

\section{ELECTRIC RELIABILITY}

COUNCIL.................. MID-ATLANTIC AREA COUNCIL

\section{UTILITY \& CONTRACTOR INFORMATION}

UTILITY

LICENSEE.

CORPORATE ADDRESS

PECO ENERGY COMPANY

2301 MARKET STREET

PHILADELPHIA, PENNSYlVANIA 19154

\section{CONTRACTOR}

ARCHITECT/ENGINEER.

BECHTEL

NUC STEAM SYS SUPPLIER

GENERAL ELECTRIC

CONSTRUCTOR

BECHTEL

TURBINE SUPPLIER

GENERAL ELECTRIC

\section{REGULATORY INFORMATION}

IE REGION RESPONSIBLE.........

IE RESIDENT INSPECTOR......... MEIL PERRY

LICENSING PROJ MANAGER......... FRANK RIMALDI

DOCKET NUMBER............ 50.353

LiCENSE \& Date ISSUANCE........ NPF 085, AUGUST 25, 1989 
1. Docket: $50-309$

2. Reporting Period: DECEMBER 1994

OPERATING STATUS

Outage + On-Line Hrs: $\quad 744.0$

3. Utility Contact: S. J. BAILEY (207) 798-4241

4. Licensed Thermal Power (MWt):

5. Nameplate Rating (Gross MUe):

6. Design Electrical Rating (Net MHe):

7. Maximum Dependable Capacity (Gross MWe):

8. Maximum Dependable Capacity (Net MWe):

9. If Changes Occurred Above Since Last Report, Give Reasons:

10. Power Level To which Restricted, If Any (Net MWe):

11. Reasons For Restrictions, If Any:

\begin{tabular}{|c|c|c|c|}
\hline & MONTH & YEAR & CUAULATIVE \\
\hline 12. Report Period Hrs & 744.0 & $8,760.0$ & $188,810.0$ \\
\hline 13. Hours Reactor Critical & 744.0 & $7,960.1$ & $156,717.1$ \\
\hline 14. Rx Reserve Shtdwn Hrs & 0.0 & 0.0 & 0.0 \\
\hline 15. Hrs Generator On-Line & 744.0 & $7,740.1$ & $152,205.1$ \\
\hline 16. Unit Reserve Shtdwn Hrs & 0.0 & 0.0 & 0.0 \\
\hline 17. Gross Therm Ener (MWH) & $2,008,346.0$ & $20,432,629.0$ & $361,328,773.0$ \\
\hline 18. Gross Elec Ener (MWH) & $681,036.0$ & $6,857,273.0$ & $119,081,843.0$ \\
\hline 19. Net Elec Ener (MUH) & $651,707.0$ & $6,631,984.0$ & $114,283,822.0$ \\
\hline 20. Unit Service Factor & 100.0 & 88.4 & 78.4 \\
\hline 21. Unit Avail Factor & 100.0 & 88.4 & 78.4 \\
\hline 22. Unit Cap Factor (MDC Net) & 101.9 & 88.0 & 72.9 \\
\hline 23. Unit Cap Factor (DER Net) & 100.7 & 87.0 & 71.3 \\
\hline 24. Unit Forced Outage Rate & 0.0 & 11.6 & 7.4 \\
\hline 25. Forced Outage Hours & 0.0 & 1019.9 & $12,584.9$ \\
\hline
\end{tabular}

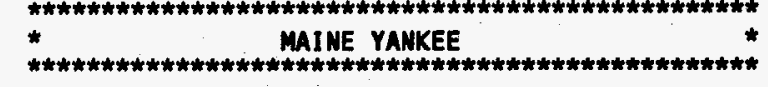

AVERAGe DAILY POUER LeVEL (Net MHe)

\begin{tabular}{ll} 
DAY & POWER \\
\hline & 876 \\
\hline 2 & 877 \\
\hline 3 & 877 \\
\hline 4 & 876 \\
\hline 5 & 877 \\
\hline 6 & 877 \\
\hline 7 & 876 \\
\hline 8 & 877 \\
\hline 9 & 875 \\
\hline 10 & 876 \\
\hline 11 & 876 \\
\hline 12 & 876 \\
\hline 13 & 876 \\
\hline 14 & 874 \\
\hline 15 & 875 \\
\hline
\end{tabular}

\begin{tabular}{|c|c}
\hline DAY & POWER \\
\hline 16 & 875 \\
\hline 17 & 877 \\
\hline 18 & 878 \\
\hline 19 & 877 \\
\hline 20 & 873 \\
\hline 21 & 878 \\
\hline 22 & 876 \\
\hline 23 & 875 \\
\hline 24 & 880 \\
\hline 25 & 872 \\
\hline 26 & 880 \\
\hline 27 & 874 \\
\hline 28 & 876 \\
\hline 29 & 875 \\
\hline 30 & 874 \\
\hline 31 & 876 \\
\hline
\end{tabular}

26. Shutdowns scheduled Over Next Six Months (Type, Date, Duration): REFUELIMg OUTAGe, februARY 25, 1995, EIGHT WEEKS.

27. If Currently Shutdown, Estimated Startup Date:

Notes:

CUMULATIVE UNIT SERVICE, AVAILABILITY, AND CAPACITY FACTORS (NDC \& DER) ARE CALCULATED WITH DATA SINCE INITIAL PHASE. CUMULATIVE UNIT CAPACITY FACTORS (MDC \& DER) ARE CALCULATED WITH WEIGHTED

AVERAGES. 


\begin{tabular}{|c|c|c|c|c|c|c|c|c|c|}
\hline No. & Date & Type & Hours & Reason & Method & LER Number & System & Component & Cause and Corrective Action To Prevent Recurrence \\
\hline
\end{tabular}

\begin{tabular}{l} 
TYPE \\
\hline F: Forced \\
S: Scheduled
\end{tabular}

\section{REASON}

A-Equipment Failure

B-Maintenance or Test

C-Refuel ing

D-Regulatory Restriction

E-Operator Training \&

License Examination

F-Administrat ive

G-Operational Error

H-other

\begin{tabular}{l} 
METHOD \\
\hline 1-Manual \\
2-Manual Scram \\
3-Auto Scram \\
4-Cont inued \\
5-Reduced Load \\
9-Other
\end{tabular}

\section{SYSTEM}

IEEE Standard

805-1984 and/o

NUREG-0161 Exhibit $F$

\section{COMPONENT}

IEEE Standard

803A-1983 and/or

NUREG-0161 Exhibit H 


\section{FACILITY DESCRIPTION}

\section{LOCATION}

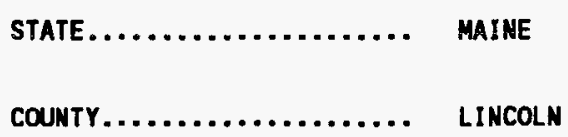

DIST AND DIRECTION FRON

MEAREST POPULATION CTR....

TYPE OF REACTOR

10 MI N OF BATH, ME

\section{PUR}

DATE INITIAL CRITICALITY...... OCTOBER 23, 1972

DATE INITIAL ELECTRICITY...... NOVEMBER DB, 1972

DATE COMMERCIAL OPERATE....... DECEMBER 28, 1972

CONDENSER COOLING METHOD...... ONCE THRU

CONDENSER COOLING WATER....... BACK RIVER

ELECTRIC RELIABILITY

COUNCIL RELIABILITY NORTHEASTERN POWER COORDINATION COUNCIL

\section{UTILITY \& CONTRACTOR INFORMATION}

UTILITY

LICENSEE

CORPORATE ADDRESS

\section{CONTRACTOR}

ARCHITECT/ENGINEER

NUC STEAM SYS SUPPLIER.

CONSTRUCTOR.

MAINE YANKEE

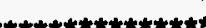

maine YANKeE atOMIC PONER CO.

83 EDISON DRIVE

AUGUSTA, MAINE 04330

TURBINE SUPPLIER

REGULATORY INFORMATION

IE REGION RESPONSIBLE......... 1

IE RESIDENT INSPECTOR.......... JIMI YEROKUN

LICENSING PROJ MANAGER......... E. H. TROTTIER

DOCKET MUMBER............. 50-309

LICENSE \& DATE ISSUANCE........ OPR 036, JUNE 29, 1973 
1. Docket: $50-369$

OPERATING STATUS

2. Reporting Period: DECEMBER 1994 Outage + On-Line Hrs: $\mathbf{7 4 4 . 0}$

3. Utility Contact: R. A. WILLIAMS (704) 382-5346

4. Licensed Thermal Power (MWt):

5. Nameplate Rating (Gross MWe):

6. Design Electrical Rating (Net MHe):

7. Maximm Dependable Capacity (Gross Mue):

8. Maximum Dependable Capacity (Net MWe):

9. If Changes Occurred Above Since Last Report, Give Reasons:

10. Power Level To Which Restricted, If Any (Net MHe):

11. Reasons For Restrictions, If Any:

\begin{tabular}{|c|c|c|c|}
\hline & MONTH & YEAR & CUMULATIVE \\
\hline Report Period Hrs & 744.0 & $8,760.0$ & $114,696.0$ \\
\hline - Hours Reactor Critical & 744.0 & $6,338.9$ & $80,359.6$ \\
\hline Rx Reserve Shtdin Hrs & 0.0 & 0.0 & 0.0 \\
\hline - Hrs Generator On-Line & 744.0 & $6,293.1$ & 79.512 .7 \\
\hline Unit Reserve Shtdwn Hrs & 0.0 & 0.0 & 0.0 \\
\hline - Gross Therm Ener (MWH) & $2,532,823.0$ & $21,097,590.0$ & $247,822,426.0$ \\
\hline - Gross Elec Ener (MWH) & $879,025.0$ & $7,189,568,0$ & $85,151,723.0$ \\
\hline - Net Elec Ener (MWH) & $845,036.0$ & $6,873,218.0$ & $81,307,368.0$ \\
\hline Unit Service factor & 100.0 & 71.8 & 69.3 \\
\hline Unit Avail Factor & 100.0 & 71.8 & 69.3 \\
\hline - Unit Cap factor (MDC Net) & 100.6 & 69.5 & 61.7 \\
\hline - Unit Cap factor (DER Net) & 96.3 & 66.5 & 60.1 \\
\hline Unit Forced Outage Rate & 0.0 & 16.2 & 14.5 \\
\hline Forced Outage Hours & 0.0 & 1218.7 & $13,457.3$ \\
\hline
\end{tabular}

$* * * * * * * * * * * * * * * * * * * * * * * * * * * * * * * * * * * * * * * * * * * * * * * * * * *$
MCGUIRE 1

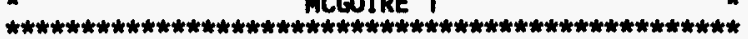

AVERAGE DAILY POWER LEVEL (Het MWe)

\begin{tabular}{ll} 
DAY & PONER \\
\hline 1 & 1134 \\
\hline 2 & 1134 \\
\hline 3 & 1135 \\
\hline 4 & 1135 \\
\hline 5 & 1135 \\
\hline 6 & 1135 \\
\hline 7 & 1135 \\
\hline 8 & 1134 \\
\hline 9 & 1134 \\
\hline 10 & 1135 \\
\hline 11 & 1136 \\
\hline 12 & 1135 \\
\hline 13 & 1135 \\
\hline 14 & 1136 \\
\hline 15 & 1137 \\
\hline
\end{tabular}

\begin{tabular}{ll} 
DAY & PONER \\
\hline 16 & 1136 \\
\hline 17 & 1137 \\
\hline 18 & 1137 \\
\hline 19 & 1136 \\
\hline 20 & 1136 \\
\hline 21 & 1135 \\
\hline 22 & 1135 \\
\hline 23 & 1136 \\
\hline 24 & 1136 \\
\hline 25 & 1137 \\
\hline 26 & 1136 \\
\hline 27 & 1137 \\
\hline 28 & 1138 \\
\hline 29 & 1138 \\
\hline 30 & 1137 \\
\hline 31 & 1138 \\
\hline
\end{tabular}

26. Shutdowns Scheduled Over Mext Six Months (Type, Date, Duration):

27. If Currently Shutdown, Estimated Startup Date:

Notes:

CUMULATIVE UNIT CAPACITY FACTOR (MDC NET) IS CALCULATED MITH A WEIGHTED AVERAGE. 
REASON

F: Forced

S: Scheduled
A-Equipment Failure

B-Maintenance

B-Maintenance

C-Refueling

- Operator Training \&

F-Adninistrative

G-Operational Error

H-Other

\begin{tabular}{l} 
METHOD \\
\hline 1-Manual \\
2-Manual Scram \\
3-Auto Scram \\
4-Cont inued \\
5-Reduced Load \\
9-0ther
\end{tabular}

SYSTEM

IEEE Standard

$805-1984$ and/or

COMPONENT

IEEE Standard

803A-1983 and/or

NUREG-0161 Exhibit H 


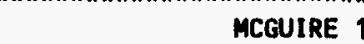

MCCUIRE 1

FACILITY DESCRIPTION

\section{LOCATION}

STATE.

countr.

NORTH CAROLINA

MECKLENBURG

\section{DIST AND DIRECTION FRON} MEAREST POPULATION CTR.

TYPE OF REACTOR.

DATE INITIAL CRITICALITY...... AUGUST 08, 1981

DATE INITIAL ELECTRICITY...... SEPTEMBER 12, 1981

date COMmercial operate....... December 01, 1981

CONDENSER COOLING METHOD.

CONDENSER COOLING WATER

ELECTRIC RELIABILITY

COUNCIL................... SOUTHEASTERN ELECTRIC

RELIABILITY COUNCIL

\section{UTILITY \& CONTRACTOR INFORMATION}

UTILITY

LICENSEE.

DUKE PONER CO.

CORPORATE ADDRESS.

422 SOUTH CHURCH STREET

CHARLOTTE, NORTH CAROLINA 28242

\section{CONTRACTOR}

ARCHITECT/ENGINEER

DUKE POWER

NUC STEAM SYS SUPPLIER

MESTINGHOUSE

CONSTRUCTOR

DUKE POWER

TURBINE SUPPLIER

WESTINGHOUSE

\section{REGULATORY INFORMATION}

IE REGION RESPONSIBLE.

2

IE RESIDENT INSPECTOR.

GEORGE MAXUELL

LICENSING PROJ MANAGER.

VICTOR NERSES

DOCKET NUMBER.

50-369

LICENSE \& DATE ISSUANCE.
NPF 009, JULY 08, 1981 
1. Docket: $50-370$

OPERATING STATUS

2. Reporting Period: DECEMBER 1994

Outage + On-Line Hrs: $\mathbf{7 4 4 . 0}$

3. Utility Contact: R. A. MILLIAMS (704) 382-5346

4. Licensed Thermal Power (MUt):

5. Nameplate Rating (Gross MHe):

6. Design Electrical Rating (Net MHe):

7. Maximum Dependable Capacity (Gross MWe):

8. Maximum Dependable Capacity (Net MWe):

9. If Changes Occurred Above Since Last Report, Give Reasons:

10. Power Level To Which Restricted, If Any (Net MHe):

11. Reasons for Restrictions, If Any:

\begin{tabular}{|c|c|c|c|}
\hline & MONTH & YEAR & CUMULATIVE \\
\hline 12. Report Period Hrs & 744.0 & $8,760.0$ & $94,992.0$ \\
\hline 13. Hours Reactor Critical & 0.0 & 7.710 .8 & $73,552.8$ \\
\hline 14. Rx Reserve Shtdun Hrs & 0.0 & 0.0 & 0.0 \\
\hline 15. Hrs Generator On-Line & 0.0 & $7,708.5$ & $72,584.8$ \\
\hline 16. Unit Reserve Shtdwn Hrs & 0.0 & 0.0 & 0.0 \\
\hline 17. Gross Therm Ener (MUH) & 0.0 & $25,973,427.0$ & $237,845,414.0$ \\
\hline 18. Gross Elec Ener (MUH) & 0.0 & $8,998,663.0$ & $83,047,598.0$ \\
\hline 19. Net Elec Ener (MHH) & $(3,730.0)$ & $8,656,226.0$ & $79,622,346.0$ \\
\hline 20. Unit Service Factor & 0.0 & 88.0 & 76.4 \\
\hline 21. Unit Avail Factor & 0.0 & 88.0 & 76.4 \\
\hline 22. Unit Cap Factor (MDC Net) & 0.0 & 87.5 & 73.4 \\
\hline 23. Unit Cap Factor (DER Net) & 0.0 & 83.7 & 71.0 \\
\hline 24. Unit Forced Outage Rate & 0.0 & 1.8 & 6.6 \\
\hline 25. Forced Outage Hours & 0.0 & 144.9 & $5,160.4$ \\
\hline
\end{tabular}

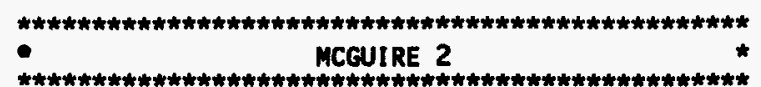

AVERAGE DAILY PONER LEVEL (Net MHe)

\begin{tabular}{lr} 
DAY & PONER \\
\hline 1 & 0 \\
\hline 3 & 0 \\
\hline 4 & 0 \\
\hline 5 & 0 \\
\hline 6 & 0 \\
\hline 7 & 0 \\
\hline 8 & 0 \\
\hline 9 & 0 \\
\hline 10 & 0 \\
\hline 11 & 0 \\
\hline 12 & 0 \\
\hline 13 & 0 \\
\hline 14 & 0 \\
\hline 15 & 0 \\
\hline
\end{tabular}

\begin{tabular}{cc} 
DAY & PONER \\
\hline 16 & 0 \\
\hline 17 & 0 \\
\hline 18 & 0 \\
\hline 19 & 0 \\
\hline 20 & 0 \\
\hline 21 & 0 \\
\hline 22 & 0 \\
\hline 23 & 0 \\
\hline 24 & 0 \\
\hline 25 & 0 \\
\hline 26 & 0 \\
\hline 27 & 0 \\
\hline 28 & 0 \\
\hline 29 & 0 \\
\hline 30 & 0 \\
\hline 31 & 0 \\
\hline
\end{tabular}

26. Shutdowns Scheduled Over Next Six Months (Type, Date, Duration):

27. If Currently Shutdown, Estimated Startup Date: 01/12/95 Notes:

CUMULATIVE UNIT CAPACITY FACTOR (MDC MET) IS CALCULATED MITH a WEI GHTED AVERAGE. 


\begin{tabular}{cccccccccc} 
No. & Date & Type & Hours & Reason & Method & LER Number & System & Component & Cause and Corrective Action To Prevent Recurrence \\
\hline 3 & $11 / 24 / 94$ & S & 744.0 & C & 4 & RC & FUELXX & REFUELING OUTAGE CONTIMUED.
\end{tabular}

\section{TYPE}

F: Forced

S: Scheduled

\section{REASON}

A-Equipment Failure

B-Maintenance or Test

C-Refuel ing

D-Regulatory Restriction

E-Operator Training \&

License Examination

G-Operational Error

-other

\section{METHOD}

1-Manual

2-Manual Scram

3-Auto Scram

5-Cont inued

9-0ther

\begin{tabular}{|c|c|}
\hline SYSTEM & COMPONENT \\
\hline $\begin{array}{l}\text { IEEE Standard } \\
805-1984 \text { and/or } \\
\text { NUREG-0161 Exh ibit F }\end{array}$ & $\begin{array}{l}\text { IEEE Standard } \\
803 A-1983 \text { and/or } \\
\text { MUREG-0161 Exh ibit H }\end{array}$ \\
\hline
\end{tabular}

IEEE Standard

MUREG-0161 Exhibit
IEEE Standard

MUREG-0161 Exhibit 


\section{FACILITY DESCRIPTION}

LOCATION

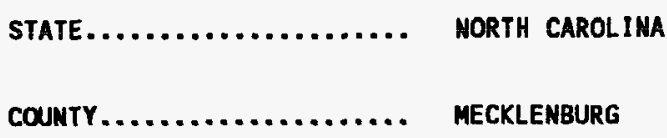
RELIABILITY COUNCIL

\section{UTILITY \& CONTRACTOR INFORMATION}

UTILITY

LICENSEE

DUKE PONER CO.

CORPORATE ADDRESS

422 SOUTH CHURCH STREET CHARLOTTE, NORTH CAROLINA 28242

\section{CONTRACTOR}

ARCHITECT/ENGINEER.

DUKE POWER

NUC STEAM SYS SUPPLIER

MEST INGHOUSE

CONSTRUCTOR

DUKE POWER

TURB INE SUPPLIER

WEST INGHOUSE

\section{REGULATORY INFORMATION}

IE REGION RESPONSIBLE......... 2

IE RESIDENT INSPECTOR.......... GEORGE MAXUELL

LICENSING PROJ MANAGER......... VICTOR NERSES

DOCKET MUMBER.............. 50-370

LICENSE \& DATE ISSUANCE. 
1. Docket: $50-245$

OPERATING STATUS

2. Reporting Period: DECEMBER 1994 Outage + On-Line Hrs: $\mathbf{7 4 4 . 0}$

3. Utility Contact: G. MEUBURGH (203) 447-1791 EXT. 5730

4. Licensed Thermal Power (MWt):

5. Nameplate Rating (Gross MWe):

6. Design Electrical Rating (Het MWe):

7. Maximum Dependable Capacity (Gross MWe):

8. Maximum Dependable Capacity (Net MUe):

9. If Changes Occurred Above since Last Report, Give Reasons:

10. Power Level To Which Restricted, If Any (Net MWe):

11. Reasons for Restrictions, If Any:

\begin{tabular}{c}
\hline 2011 \\
\hline 662 \\
\hline 660 \\
\hline 670 \\
\hline 641 \\
\hline
\end{tabular}
.

\begin{tabular}{|c|c|c|c|}
\hline & MONTH & YEAR & CUMULATIVE \\
\hline 12. Report Period Hrs & 744.0 & $8,760.0$ & $211,176.0$ \\
\hline 13. Hours Reactor Critical & 687.4 & $5,575.0$ & $163,525.6$ \\
\hline 14. Rx Reserve Shtdwn Krs & 0.0 & 0.0 & $3,283.3$ \\
\hline 15. Hrs Generator On-Line & 665.8 & $5,413.3$ & $159,628.3$ \\
\hline 16. Unit Reserve Shtdwn Hrs & 0.0 & 0.0 & 93.7 \\
\hline 17. Gross Therm Ener (MUH) & $1,292,114.0$ & $10,264,679.0$ & $300,810,521.0$ \\
\hline 18. Gross Elec Ener (MWH) & $437,882.0$ & $3,451,697.0$ & $101,378,255.0$ \\
\hline 19. Net Elec Ener (MWH) & $418,523.0$ & $3,286,473.0$ & $96,712,607.0$ \\
\hline 20. Unit Service Factor & 89.5 & 61.8 & 75.6 \\
\hline 21. Unit Avail Factor & 89.5 & 61.8 & 75.6 \\
\hline 22. Unit Cap Factor (MDC Net) & 87.8 & 58.5 & 70.1 \\
\hline 23. Unit Cap Factor (DER Net) & 85.2 & 56.8 & 69.4 \\
\hline 24. Unit Forced Outage Rate & 10.5 & 4.8 & 11.9 \\
\hline 25. Forced Outage Hours & 78.2 & 271.6 & $21,491.5$ \\
\hline
\end{tabular}

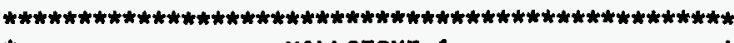 * MILLSTONE 1 P}

AVERAGe dAILY POWER LeVel (Net MHe)

\begin{tabular}{lr} 
DAY & PONER \\
\hline 1 & 0 \\
\hline 2 & 0 \\
\hline 3 & 0 \\
\hline 4 & 152 \\
\hline 5 & 477 \\
\hline 6 & 563 \\
\hline 7 & 623 \\
\hline 8 & 649 \\
\hline 9 & 653 \\
\hline 10 & 653 \\
\hline 11 & 654 \\
\hline 12 & 653 \\
\hline 13 & 653 \\
\hline 14 & 642 \\
\hline 15 & 653 \\
\hline
\end{tabular}

\begin{tabular}{ll} 
DAY & PONER \\
\hline 16 & 653 \\
\hline 17 & 653 \\
\hline 18 & 653 \\
\hline 19 & 654 \\
\hline 20 & 654 \\
\hline 21 & 647 \\
\hline 22 & 653 \\
\hline 23 & 654 \\
\hline 24 & 653 \\
\hline 25 & 654 \\
\hline 26 & 653 \\
\hline 27 & 654 \\
\hline 28 & 645 \\
\hline 29 & 653 \\
\hline 30 & 653 \\
\hline 31 & 653 \\
\hline
\end{tabular}

26. Shutdowns Scheduled Over Mext Six Months (Type, Date, Duration):

27. If Currently Shutdown, Estimated Startup Date:

Notes:

CUMULATIVE UNIT CAPACITY FACTOR (MDC NET) IS CALCULATED UITH A WEIGHTED AVERAGE. 
$94-084 \quad 11 / 24 / 94$

$$
78.2
$$

A

4

94033

SB

RV

SAFETY RELIEF VALVE LEAKAGE RESULTING IN SETPOINT DRIFT.

\begin{tabular}{|c|c|}
\hline TYPE & REASON \\
\hline $\begin{array}{l}\text { F: Forced } \\
\text { S: Scheduled }\end{array}$ & $\begin{array}{l}\text { A-Equipment Failure } \\
\text { B-Maintenance or rest } \\
\text { C-Refuel ing } \\
\text { D-Regulatory Restriction } \\
\text { E-Operator Training \& } \\
\text { License Examination } \\
\text { F-Administrative } \\
\text { G-Operational Error } \\
\text { H-Other }\end{array}$ \\
\hline
\end{tabular}

METHOD

1-Manual

2-Manual Scram

3-Auto Scram

4-Cont inued

5 -Reduced Load

9-0ther \begin{tabular}{l} 
SYSTEM \\
\hline IEEE Standard \\
$805-1984$ and/or \\
MUREG-0161 Exhibit $F$
\end{tabular}
COMPONENT

IEEE Standard 803A-1983 and/or NUREG-0161 Exhibit H

F-Administrative

$H$-Other 


\section{FACILITY DESCRIPIION}

\section{LOCATION}

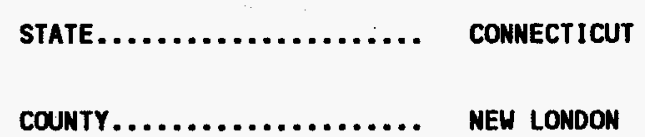

\section{DIST ANO DIRECTION FRON} MEAREST POPULATION CTR.

TYPE OF REACTOR.

3.2 MI ENE OF MEW LONDON, CT

BUR

DATE INITIAL CRITICALITY...... OCTOBER 26, 1970

DATE INITIAL ELECTRICITY...... NOVEMBER 29, 1970

DATE COMMERCIAL OPERATE....... MARCh 01, 1971

CONDENSER COOLING METHOD...... ONCE THRU

CONDENSER COOLING MATER....... LONG ISLAND SOUND

ELECTRIC RELIABILITY

COUNCIL $\ldots \ldots \ldots \ldots \ldots \ldots \ldots \ldots$ NORTHEASTERN POUER

COORDINATION COUNCIL

\section{UTILITY \& CONTRACTOR INFORMATION}

UTILITY

LICENSEE.

NORTHEAST UTILITIES

CORPORATE ADDRESS

P.0. BOX 270

HARTFORD, CONNECTICUT 061410270

\section{CONTRACTOR}

ARCHITECT/ENGINEER......... EBASCO

NUC STEAM SYS SUPPLIER...... GENERAL ELECTRIC

CONSTRUCTOR................ EBASCO

TURBIME SUPPLIER........... GENERAL ELECTRIC

\section{REGULATORY INFORMATION}

IE REGION RESPONSIBLE......... 1

IE RESIDENT IMSPECTOR.......... PAUL D. SWETLAND

LICENSING PROJ MANAGER........ JAMES $W$. ANDERSEN

DOCKET NUMBER............. $50-245$

LICENSE \& DATE ISSUANCE........ DPR 021, OCTOBER 26, 1970 


\section{Docket: $50-336$}

OPERATING STATUS

2. Reporting Period: DECEMBER 1994

Outage + On-Line Hrs: 744.0

3. Utility Contact: R. BORCHERT (203) 447-1791 EXT. 4418

4. Licensed Thermal Power (MUt):

5. Nameplate Rating (Gross MWe):

6. Design Electrical Rating (Net MHe):

7. Maximun Dependable Capacity (Gross MHe):

8. Maximum Dependable Capacity (Net MHe):

9. If Changes Occurred Above Since Last Report, Give Reasons:

10. Power Level To Which Restricted, If Any (Net MWe):

11. Reasons For Restrictions, If Any:

\begin{tabular}{|c|c|c|c|}
\hline & MONTH & YEAR & CUMULATIVE \\
\hline 12. Report Period Hrs & 744.0 & $8,760.0$ & $166,704.0$ \\
\hline 13. Hours Reactor Critical & 0.0 & $4,349.2$ & $117,296.7$ \\
\hline 14. Rx Reserve Shtdun Hrs & 0.0 & 0.0 & $2,205.5$ \\
\hline 15. Hrs Generator On-Line & 0.0 & $4,291.7$ & $112,106.4$ \\
\hline 16. Unit Reserve Shtdun Hrs & 0.0 & 0.0 & 468.2 \\
\hline 17. Gross Therm Ener (MUH) & 0.0 & $11,426,394.0$ & $289,026,547.4$ \\
\hline 18. Gross Elec Ener (MUH) & 0.0 & $3,820,736.0$ & $94,772,725.0$ \\
\hline 19. Net Elec Ener (MWH) & $(3,345.0)$ & $3,658,789.0$ & $90,905,587.8$ \\
\hline 20. Unit Service Factor & 0.0 & 49.0 & 67.2 \\
\hline 21. Unit Avail Factor & 0.0 & 49.0 & 67.5 \\
\hline 22. Unit Cap Factor (MDC Net) & 0.0 & 47.8 & 63.8 \\
\hline 23. Unit Cap factor (DER Net) & 0.0 & 48.0 & 62.8 \\
\hline 24. Unit Forced Outage Rate & 0.0 & 17.4 & 15.3 \\
\hline 25. Forced Outage Hours & 0.0 & 903.8 & $20,244.8$ \\
\hline
\end{tabular}

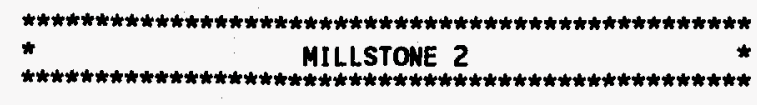

AVERAGE DAILY POWER LEVEL (Het MWe)

\begin{tabular}{|c|c|c|c|}
\hline DAY & POWER & DAY & POWER \\
\hline 1 & 0 & 16 & 0 \\
\hline 2 & 0 & 17 & 0 \\
\hline 3 & 0 & 18 & 0 \\
\hline 4 & 0 & 19 & 0 \\
\hline 5 & 0 & 20 & 0 \\
\hline 6 & 0 & 21 & 0 \\
\hline 7 & 0 & 22 & 0 \\
\hline 8 & 0 & 23 & 0 \\
\hline 9 & 0 & 24 & 0 \\
\hline 10 & 0 & 25 & 0 \\
\hline 11 & 0 & 26 & 0 \\
\hline 12 & 0 & 27 & 0 \\
\hline 13 & 0 & 28 & 0 \\
\hline 14 & 0 & 29 & 0 \\
\hline \multirow[t]{2}{*}{15} & 0 & 30 & 0 \\
\hline & & 31 & 0 \\
\hline
\end{tabular}

26. Shutdowns Scheduled Over Mext Six Months (Type, Date, Duration):

27. If Currently Shutdown, Estimated Startup Date:

Notes:

CUMULATIVE UNIT CAPACITY FACTORS (MDC \& DER) ARE CALCULATED WITH WEIGHTED AVERAGES. 


No. Date Type Hours Reason Method LER Number System Component Cause and Corrective Action To Prevent Recurrence

$\begin{array}{llllllll}94-04 & 10 / 01 / 94 & S & 744.0 & C & 4 & \text { RC } & \text { FUELXX } \\ \text { REFUELING OUTAGE CONTINUED. }\end{array}$

\begin{tabular}{|c|c|}
\hline TYPE & REASON \\
\hline $\begin{array}{l}\text { F: Forced } \\
\text { S: Scheduled }\end{array}$ & $\begin{array}{l}\text { A-Equipment Failure } \\
\text { B-Maintenance or Test } \\
\text { C-Refuel ing } \\
\text { D-Regulatory Restriction } \\
\text { E-Operator Training \& } \\
\text { License Examination } \\
\text { F-Administrat ive } \\
\text { G-Operational Error } \\
\text { H-Other }\end{array}$ \\
\hline
\end{tabular}

\begin{tabular}{l} 
METHOD \\
\hline 1-Manual \\
2-Manual Scram \\
3-Auto Scram \\
4-Cont inued \\
5-Reduced Load \\
9-0ther
\end{tabular}

SYSTEM COMPONENT

IEEE Standard

$805-1984$ and/or

NUREG-0161 Exhibit $F$

IEEE Standard

803A-1983 and/or
NUREG-0161 Exhibit H

-Reduced Load

F-Administrative

$H$-other 
FACILITY DESCRIPTION

LOCATION

STATE................. CONMECTICUT

COUNTY................. NEW LONDON

\section{DIST AND DIRECTION FROM}

NEAREST POPULATION CTR.... 3.2 MI ENE OF NEH LONDON, CT

TYPE OF REACTOR

PUR

DATE INITIAL CRITICALITY...... OCTOBER 17, 1975

DATE IHITIAL ELECTRICITY...... NOVEMBER 09, 1975

DATE COMmERCIAL OPERATE....... DECEMBER 26, 1975

CONDENSER COOLIMG METHOD...... ONCE THRU

CONDENSER COOLING WATER....... LONG ISLAMD SOUND

ELECTRIC RELIABILITY

COUNCIL................... NORTHEASTERN POWER COORDINATION COUNCIL

\section{UTILITY \& CONTRACTOR INFORMATION}

UTILITY

LICENSEE................ NORTHEAST UTILITIES

CORPORATE ADDRESS........... P.O. BOX 270

P.O. BOX 270
HARTFORD, CONNECTICUT 061410270

\section{CONTRACTOR}

ARCH ITECT/ENGINEER......... BECHTEL

MUC STEAM SYS SUPPLIER.

COMBUSTION EMGINEERING

CONSTRUCTOR.

BECHTEL

TURBINE SUPPLIER

GEMERAL ELECTRIC

\section{REGULATORY INFORMATION}

IE REGION RESPONSIBLE......... 1

IE RESIDENT INSPECTOR

PAUL D. SWETLAND

LICENSING PROJ MAHAGER

GUY S. VISSING

DOCKET NUMBER

50-336

LICENSE \& DATE ISSUANCE 
1. Docket: $50-423$

2. Reporting Period: DECEMBER 1994

OPERATING

S T A T U S

3. Utility Contact: I. R. HUDSON (203) 444-5400

4. Licensed Thermal Power (MWt):

5. Nameplate Rating (Gross MWe):

6. Design Electrical Rating (Net MWe):

7. Maximm Dependable Capacity (Gross MWe):

8. Maximum Dependable Capacity (Net MHe):

9. If Changes Occurred Above Since Last Report, Give Reasons:

10. Power Level To Which Restricted, If Any (Net MWe):

11. Reasons For Restrictions, If Any:

\begin{tabular}{|c|c|c|c|}
\hline & MONTH & YEAR & CUMULATIVE \\
\hline 12. Report Period Hrs & 744.0 & $8,760.0$ & $76,200.0$ \\
\hline 13. Hours Reactor Critical & 744.0 & $8,454.7$ & $57,768.6$ \\
\hline 14. Rx Reserve Shtdwn Hrs & 0.0 & 0.0 & $6,459.1$ \\
\hline 15. Hrs Generator on-Line & 744.0 & $8,426.1$ & $56,663.7$ \\
\hline 16. Unit Reserve Shtdun Hrs & 0.0 & 0.0 & 0.0 \\
\hline 17. Gross Therm Ener (MWH) & $2,533,923.0$ & $28,490,276.0$ & $185,775,382.2$ \\
\hline 18. Gross Elec Ener (MUH) & $886,773.0$ & $9,815,466.0$ & $63,983,111.1$ \\
\hline 19. Net Elec Ener (MUH) & $852,243.2$ & $9,416,153.8$ & $60,882,759.1$ \\
\hline 20. Unit Service Factor & 100.0 & 96.2 & 74.4 \\
\hline 21. Unit Avail Factor & 100.0 & 96.2 & 74.4 \\
\hline 22. Unit Cap Factor (MDC Net) & 100.7 & 94.5 & 70.1 \\
\hline 23. Unit Cap Factor (DER Net) & 99.3 & 93.1 & 69.2 \\
\hline 24. Unit Forced Outage Rate & 0.0 & 3.8 & 15.4 \\
\hline 25. Forced Outage Hours & 0.0 & 333.9 & $10,338.8$ \\
\hline
\end{tabular}

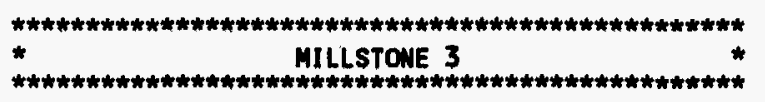

AVERAGE DAILY POWER LEVEL (Net me)

\begin{tabular}{ll} 
DAY & POWER \\
\hline 1 & 1147 \\
\hline 2 & 1147 \\
\hline 3 & 1147 \\
\hline 4 & 1146 \\
\hline 5 & 1148 \\
\hline 6 & 1147 \\
\hline 7 & 1144 \\
\hline 8 & 1146 \\
\hline 9 & 1148 \\
\hline 10 & 1147 \\
\hline 11 & 1147 \\
\hline 12 & 1147 \\
\hline 13 & 1146 \\
\hline 14 & 1145 \\
\hline 15 & 1147 \\
\hline
\end{tabular}

\begin{tabular}{ll} 
DAY & PONER \\
\hline 16 & 1146 \\
\hline 17 & 1103 \\
\hline 18 & 1146 \\
\hline 19 & 1143 \\
\hline 20 & 1148 \\
\hline 21 & 1147 \\
\hline 22 & 1148 \\
\hline 23 & 1147 \\
\hline 24 & 1148 \\
\hline 25 & 1147 \\
\hline 26 & 1149 \\
\hline 27 & 1149 \\
\hline 28 & 1150 \\
\hline 29 & 1148 \\
\hline 30 & 1149 \\
\hline 31 & 1144 \\
\hline
\end{tabular}

26. Shutdowns Scheduled Over Next six Months (Type, Date, Duration): REFUELING OUTAGE, APRIL 8, 1995, 54 DAYS.

27. If Currently Shutdown, Estimated Startup Date:

Notes:

CUMULATIVE UNIT CAPACITY FACTOR (MDC NET) IS CALCULATED WITH A WEIGHTED AVERAGE. 


\begin{tabular}{llllllll}
\hline No. Date & Type Hours Reason Method LER Number System Component Cause and Corrective Action To Prevent Recurrence \\
\hline
\end{tabular}

\begin{tabular}{l} 
TYPE \\
\hline F: Forced \\
S: Scheduled
\end{tabular}

\section{REASON}

A-Equipment Failure

B-Maintenance or Test

C-Refuel ing

D-Regulatory Restriction

E-Operator Training \&

License Examination

F-Administrative

G-Operational Error

H-Other

\section{SYSTEM}

IEEE Standard

NUREG-0161 Exhibit F

\section{COMPONENT}

IEEE Standard

NUREG-0161 Exhibit H

3-Manual Scram

4-Auto Scram

5-Reduced Load

9-Other 


\section{FACILITY DESCRIPTION}

\section{LOCATION}

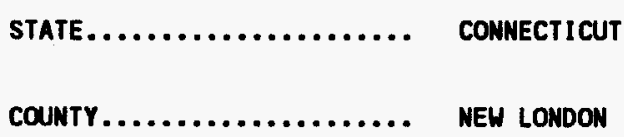

\section{DIST AND DIRECTION FRON}

MEAREST POPULATION CTR..... 3.2 MI ENE OF NEW LONDON, CT

TYPE OF REACTOR

PUR

DATE INITIAL CRITICALITY...... JANUARY 23, 1986

DATE INITIAL ELECTRICITY...... FEBRUARY 12, 1986

DATE COMmercial operate....... APRIL 23, 1986

CONDENSER COOLING METHOD...... ONCE THRU

CONDENSER COOLING WATER....... NIANTIC BAY

ELECTRIC RELIABILITY

COUNCIL................... NORTHEASTERN POWER

COORDINATION COUNCIL

\section{UTILITY \& CONTRACTOR INFORMATION}

UTILITY

LICENSEE.

MORTHEAST NUCLEAR ENERGY CO.

CORPORATE ADDRESS

P.O. BOX 270

HARTFORD, CONNECTICUT 061410270

\section{CONTRACTOR}

ARCHI TECT/ENGINEER.

STONE \& WEBSTER

NUC STEAM SYS SUPPLIER.

WEST INGHOUSE

CONSTRUCTOR

STONE \& WEBSTER

TURBINE SUPPLIER.

GEMERAL ELECTRIC

\section{REGULATORY INFORMATION}

IE REGION RESPONSIBLE......... 1

IE RESIDENT INSPECTOR.......... PAUL D. SWETLAND

LICENSING PROJ MANAGER......... VERNON L. ROONEY

DOCKET NUMBER............. 50-423

LICENSE \& DATE ISSUANCE........ NPF 049, JANUARY 31, 1986 


\section{Docket: $50-263$}

OPERATING STATUS

2. Reporting Period: DECEMBER 1994

Outage + On-Line Hrs:

744.0

3. Utility Contact:

H. H. PAUSTIAN (612) 295-5151

4. Licensed Thermal Power (MWt):

5. Nameplate Rating (Gross MWe):

6. Design Electrical Rating (Net MHe):

7. Maximum Dependable Capacity (Gross MWe):

8. Maximum Dependable Capacity (Net MWe):

\begin{tabular}{c}
1670 \\
\hline 569 \\
\hline 545 \\
\hline 564 \\
\hline 536
\end{tabular}

9. If Changes Occurred Above Since Last Report, Give Reasons:

10. Power Level To thich Restricted, If Any (Net MWe):

11. Reasons for Restrictions, If Any:

\begin{tabular}{|c|c|c|c|}
\hline 12. Report Period Hrs & $\begin{array}{l}\text { MONTH } \\
744.0\end{array}$ & $\begin{array}{l}\text { YEAR } \\
8,760.0\end{array}$ & $\begin{array}{l}\text { CUMULAT IVE } \\
206,041.0\end{array}$ \\
\hline 13. Hours Reactor Critical & 669.3 & $7,624.2$ & $166,999.9$ \\
\hline 4. Rx Reserve Shtdwn hrs & 0.0 & 0.0 & 940.7 \\
\hline 5. Hrs Generator on-Line & 642.2 & $\overline{7,510.2}$ & $164,110.9$ \\
\hline 16. Unit Reserve Shtdwn Hrs & 0.0 & 0.0 & 0.0 \\
\hline 17. Gross Therm Ener (MUH) & $1,060,277.0$ & $12,221,672.0$ & $253,486,452.0$ \\
\hline 18. Gross Elec Ener (MWH) & $364,345.0$ & $4,128,815.0$ & $85,683,478.0$ \\
\hline 19. Het Elec Ener (MHH) & $350,970.0$ & $3,956,317.0$ & $81,975,110.0$ \\
\hline 20. Unit Service Factor & 86.3 & 85.7 & 79.6 \\
\hline 21. Unit Avail Factor & 86.3 & 85.7 & 79.6 \\
\hline 22. Unit Cap factor (MDC Net) & 88.0 & 84.3 & 74.2 \\
\hline 23. Unit Cap Factor (DER Met) & 86.6 & 82.9 & 73.0 \\
\hline 24. Unit forced Outage Rate & 3.9 & 2.9 & 3.6 \\
\hline . Forced Outage Hours & 26.0 & 220.7 & $6,073.3$ \\
\hline
\end{tabular}

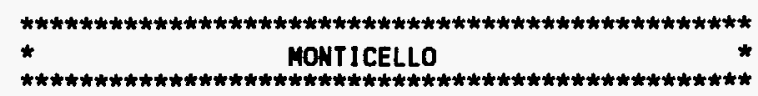

AVERAGe DAILY POWER LEVEL (Het MHe)

\begin{tabular}{lc} 
DAY & POWER \\
\hline 1 & 557 \\
\hline 2 & 554 \\
\hline 4 & 557 \\
\hline 5 & 556 \\
\hline 6 & 556 \\
\hline 7 & 556 \\
\hline 8 & 555 \\
\hline 9 & 555 \\
\hline 10 & 555 \\
\hline 11 & 555 \\
\hline 12 & 555 \\
\hline 13 & 555 \\
\hline 14 & 554 \\
\hline 15 & 554 \\
\hline
\end{tabular}

DAY PONER

\begin{tabular}{lr}
16 & 531 \\
\hline 17 & 36
\end{tabular}

$18-5$

$19-8$

\begin{tabular}{l}
20 \\
\hline 21
\end{tabular}

$21 \quad 204$

$22 \quad 552$

\begin{tabular}{ll}
\hline 23 & 555 \\
\hline 24 & 556
\end{tabular}

\begin{tabular}{ll}
24 & 556 \\
\hline 25 & 556
\end{tabular}

\begin{tabular}{ll}
25 & 556 \\
\hline 26 & 552
\end{tabular}

$27 \quad 555$

\begin{tabular}{lr}
\hline 28 & 556 \\
\hline 29 & 555
\end{tabular}

\begin{tabular}{ll}
\hline 29 & 555 \\
\hline 30 & 554
\end{tabular}

555

26. Shutdowns Scheduled Over Next Six Months (Type, Date, Duration):

27. If Currently Shutdown, Estimated Startup Date:

Motes: 


\begin{tabular}{|c|c|c|c|c|c|c|c|c|c|}
\hline No. & Date & Type & Hours & Reason & Method & LER Number & System & Component & Cause and Corrective Action To Prevent Recurrence \\
\hline 7 & $12 / 17 / 94$ & $\mathbf{s}$ & 75.8 & B & 2 & & AD & SEAL & $\begin{array}{l}\text { MAINTENANCE OUTAGE TO REPLACE REACTOR RECIRC PUMP } \\
\text { SEAL. }\end{array}$ \\
\hline 7 & $12 / 20 / 94$ & $\boldsymbol{F}$ & 26.0 & $\mathbf{8}$ & 9 & & & & $\begin{array}{l}\text { RHR/SPCI TESTABLE CHECK VALVE LEAKAGE REPAIRS } \\
\text { EXTENDED OUTAGE BEYOND RECIRC PUMP REPAIRS. }\end{array}$ \\
\hline
\end{tabular}

\begin{tabular}{|c|c|}
\hline TYPE & REASON \\
\hline $\begin{array}{l}\text { F: Forced } \\
\text { S: Scheduled }\end{array}$ & $\begin{array}{l}\text { A-Equipment Failure } \\
\text { B-Maintenance or Test } \\
\text { C-Refuel ing } \\
\text { D-Regulatory Restriction } \\
\text { E-Operator Training \& } \\
\text { License Examination } \\
\text { F-Administrative } \\
\text { G-Operational Error } \\
\text { H-Other }\end{array}$ \\
\hline
\end{tabular}

\begin{tabular}{l} 
METHCO \\
\hline 1-Manual \\
2-Manual Scram \\
3-Auto Scram \\
4-Cont inued \\
5-Reduced Load \\
9-0ther
\end{tabular}

\section{SYSTEM}

IEEE Standard

805-1984 and/or

NUREG-0161 Exhibit F
COMPONENT

IEEE Standard

803A-1983 and/or

NUREG-0161 Exhibit

G-Operational Error

Page 2-173 
FACILITY DATA

\section{FACILITY DESCRIPTION}

\section{LOCATION}

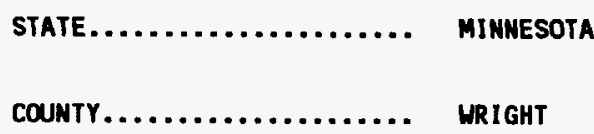

\section{DIST AND DIRECTION FROM \\ FROM}

TYPE OF REACTOR.

TYPE OF REACTOR..............

DATE INITIAL CRITICALITY......

DATE INITIAL ELECTRICITY......

DATE COMMERCIAL OPERATE.......

CONDENSER COOLING METHOD.......

CONDENSER COOLING WATER

ELECTRIC RELIABILITY

COUNCIL....................
30 MI NH OF MINNEAPOLIS, MN

\section{BUR}

DECEMBER 10, 1970

MARCH 05, 1971

JUNE 30, 1971

COOLING TOWER

MISSISSIPPI RIVER

MID-CONTINENT AREA RELIABILITY COUNCIL

\section{UTILITY \& CONTRACTOR INFORMATION}

UTILITY

LICENSEE.

CORPORATE ADDRESS.

\section{CONTRACTOR}

ARCHI TECT/ENGINEER.

MUC STEAM SYS SUPPLIER.

CONSTRUCTOR.

TURBINE SUPPLIER

REGULATORY INFORMATION

IE REGION RESPONSIBLE.

IE RESIDENT INSPECTOR.

LICENSING PROJ MANAGER.

DOCKET NUMBER

LICENSE \& DATE ISSUANCE
$* \pi *$

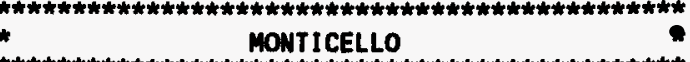


1. Docket: $50-220$

DPERATING STATUS

2. Reporting Period: DECEMBER 1994

Outage + On-Line Hrs: $\mathbf{7 4 4 . 0}$

3. Utility Contact: D. E. COLEMAN (315) 349-2558

4. Licensed Thermal Power (MWt):

5. Nameplate Rating (Gross MWe):

6. Design Electrical Rating (Net MWe):

7. Maximum Dependable Capacity (Gross MWe):

8. Maximum Dependable Capacity (Net Mue):

9. If Changes Occurred Above Since Last Report, Give Reasons:

10. Power Level To Which Restricted, If Any (Net MWe):

11. Reasons For Restrictions, If Any:

\begin{tabular}{|c|c|c|c|}
\hline & MONTH & YEAR & CUMULATIVE \\
\hline 12. Report Period Hrs & 744.0 & $8,760.0$ & $221,713.0$ \\
\hline 13. Hours Reactor Critical & 744.0 & $8,428.1$ & $146,664.3$ \\
\hline 14. Rx Reserve Shtdun Hrs & 0.0 & 0.0 & $1,204.2$ \\
\hline 15. Hrs Generator On-Line & 744.0 & $8,392.1$ & $142,839.0$ \\
\hline 16. Unit Reserve Shtdun Hrs & 0.0 & 0.0 & 20.4 \\
\hline 17. Gross Therm Ener (MUH) & $1,276,517.0$ & $15,013,536.0$ & $241,756,152.0$ \\
\hline 18. Gross Elec Ener (MUH) & $433,929.0$ & $5,053,798.0$ & $80,348,607.0$ \\
\hline 19. Net Elec Ener (MWH) & $421,522.0$ & $4,917,951.0$ & $77,880,755.0$ \\
\hline 20. Unit Service Factor & 100.0 & 95.8 & 64.4 \\
\hline 21. Unit Avail Factor & 100.0 & 95.8 & 64.4 \\
\hline 22. Unit Cap Factor (MDC Net) & 100.3 & 99.4 & 57.8 \\
\hline 23. Unit Cap Factor (DER Net) & 92.4 & 91.6 & 56.7 \\
\hline 24. Unit Forced Outage Rate & 0.0 & 4.2 & 24.0 \\
\hline 25. Forced Outage Hours & 0.0 & 367.9 & 45.110 .6 \\
\hline
\end{tabular}

\section{* NIME MILE POINT}

NINE MILE POINT 1

AVERAGE DAILY POWER LEVEL (Net MHe)

\begin{tabular}{ll} 
DAY & POWER \\
\hline 1 & 602 \\
\hline 2 & 599 \\
\hline 3 & 597 \\
\hline 4 & 594 \\
\hline 5 & 591 \\
\hline 6 & 591 \\
\hline 7 & 587 \\
\hline 8 & 586 \\
\hline 9 & 583 \\
\hline 10 & 579 \\
\hline 11 & 579 \\
\hline 12 & 575 \\
\hline 13 & 573 \\
\hline 14 & 571 \\
\hline 15 & 568 \\
\hline
\end{tabular}

\begin{tabular}{ll} 
DAY & PONER \\
\hline 16 & 566 \\
\hline 17 & 565 \\
\hline 18 & 562 \\
\hline 19 & 560 \\
\hline 20 & 558 \\
\hline 21 & 552 \\
\hline 22 & 552 \\
\hline 23 & 549 \\
\hline 24 & 550 \\
\hline 25 & 548 \\
\hline 26 & 545 \\
\hline 27 & 543 \\
\hline 28 & 540 \\
\hline 29 & 539 \\
\hline 30 & 535 \\
\hline 31 & 526 \\
\hline
\end{tabular}

26. Shutdowns Scheduled Over Mext Six Months (Type, Date, Duration): REFUELING OUTAGE, FEBRUARY 11, 1995, 40 DAYS.

27. If Currently Shutdown, Estimated Startup Date:

Notes:

CUMULATIVE UNIT CAPACITY FACTORS (MOC \& DER) ARE CALCULATED MITH WEIGHTED AVERAGES. 


\section{TYPE}

F: Forced

S: scheduled
REASON

\section{A-Equipment failure}

B-Maintenance or Test

C-Refuel ing

D-Regulatory Restriction

E-Operator Training

License Examination

F-Administrative

G-Operational Error

G-Operat
METHOD

2-Manual Scram

3-Auto Scram

4--Contin Screm

5-Reduced Load

9-0ther

\section{SYSTEM}

IEEE Standard

805-1984 and/or
NUREG-0161 Exhibit

\section{COMPONENT}

IEEE Standard

803A-1983 and/or

MUREG-0161 Exhibit 


\section{FACILITY DESCRIPTION}

LOCATION

STATE.

COUNTY.

NEW YORK

OSWEGO

\section{DIST AND DIRECTION FROM}

NEAREST POP

6 MI NE OF OSWEGO, NY

TYPE OF REACTOR.

\section{BWR}

DATE INITIAL CRITICALITY....... SEPTEMBER 05, 1969

DATE INITIAL ELECTRICITY...... NOVEMBER 09, 1969

DATE COMMERCIAL OPERATE........ DECEMBER 01, 1969

CONDENSER COOLING METHOD...... ONCE THRU

CONDENSER COOLING WATER....... LAKE ONTARIO

ELECTRIC RELIABILITY

COUMCIL $\ldots$ RELIABILITY ........ MORTHEASTERH PONER

MORTHEASTERN PONER
COORDINATION COUNCIL

\section{UTILITY \& CONTRACTOR INFORMATION}

UTILITY

LICENSEE

CORPORATE ADDRESS

\section{CONTRACTOR}

ARCHITECT/ENGINEER

NUC STEAM SYS SUPPLIER.

CONSTRUCTOR.

TURBINE SUPPLIER

REGULATORY INFORMATION

IE REGION RESPONSIBLE.......... 1

IE RESIDENT INSPECTOR.......... BARRY NORRIS

LICENSING PROJ MANAGER......... DONALD S. BRINKMAN

DOCKET NUMBER.............. $50-220$

LICENSE \& DATE ISSUANCE........ DPR 063, DECEMBER 26, 1974 
1. Docket: $50-410$

OPERATING STATUS

2. Reporting Period: DECEMBER 1994

Outage + On-Line Hrs: $\quad \mathbf{7 4 4 . 0}$

3. Utility Contact: C. J. CAROCCIO (315) 349-4615

4. Licensed Thermal Power (MWt):

5. Nameplate Rating (Gross MUe):

6. Design Electrical Rating (Net MWe):

7. Maximum Dependable Capacity (Gross MHe):

8. Maximum Dependable Capacity (Net MWe):

9. If Changes Occurred Above Since Last Report, Give Reasons:

10. Power Level To thich Restricted, If Any (Net MWe):

11. Reasons For Restrictions, If Any:

\begin{tabular}{|c|c|c|c|}
\hline & MONTH & & CUMULATIVE \\
\hline 12. Report Period Hrs & 744.0 & $8,760.0$ & $59,089.0$ \\
\hline 13. Hours Reactor Critical & 571.7 & $8,374.3$ & $41,360.4$ \\
\hline 14. Rx Reserve Shtdun Hrs & 0.0 & 0.0 & 0.0 \\
\hline 15. Hrs Generator On-Line & 502.4 & 8.243 .6 & 39.424 .8 \\
\hline 16. Unit Reserve Shtdun Hrs & 0.0 & 12.9 & 12.9 \\
\hline 17. Gross Therm Ener (MUH) & $1,619,601.0$ & $26,975,978.6$ & $\overline{123,188,979.0}$ \\
\hline 18. Gross Elec Ener (MWH) & $533,346.5$ & $8,871,403.8$ & $40,716,842.1$ \\
\hline 19. Net Elec Ener (МนH) & $501,880.7$ & $8,355,910.3$ & $38,278,706.8$ \\
\hline 20. Unit Service Factor & 67.5 & 94.1 & 66.7 \\
\hline 21. Unit Avail Factor & 67.5 & 94.3 & 66.7 \\
\hline 22. Unit Cap Factor (MDC Net) & 67.9 & 96.0 & 62.4 \\
\hline 23. Unit Cap factor (DER Net) & 63.5 & 89.8 & 60.5 \\
\hline 24. Unit Forced Outage Rate & 32.5 & 5.9 & 16.1 \\
\hline 25. Forced Outage Hours & 241.6 & 516.4 & $7,564.4$ \\
\hline
\end{tabular}

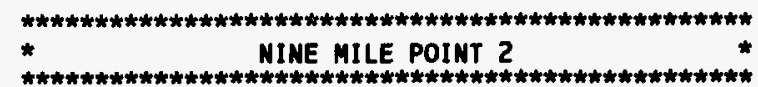

AVERAGE DAILY PONER LEVEL (Net me)

\begin{tabular}{cc} 
DAY & PONER \\
\hline 1 & 1043 \\
\hline 2 & 1040 \\
\hline 3 & 1023 \\
\hline 4 & 1037 \\
\hline 5 & 1036 \\
\hline 6 & 1039 \\
\hline 7 & 1043 \\
\hline 8 & 1045 \\
\hline 9 & 495 \\
\hline 10 & 0 \\
\hline 11 & 0 \\
\hline 12 & 0 \\
\hline 13 & 0 \\
\hline 14 & 0 \\
\hline 15 & 0 \\
\hline
\end{tabular}

\begin{tabular}{cc} 
DAY & PONER \\
\hline 16 & 0 \\
\hline 17 & 0 \\
\hline 18 & 0 \\
\hline 19 & 53 \\
\hline 20 & 685 \\
\hline 21 & 961 \\
\hline 22 & 1041 \\
\hline 23 & 1040 \\
\hline 24 & 1038 \\
\hline 25 & 1042 \\
\hline 26 & 1044 \\
\hline 27 & 1042 \\
\hline 28 & 1040 \\
\hline 29 & 1043 \\
\hline 30 & 1044 \\
\hline 31 & 1037 \\
\hline
\end{tabular}

26. Shutdowns Scheduled Over Mext Six Months (Type, Date, Duration): REFUELING OUTAGE, APRIL 8, 1995, 51 DAYS.

27. If Currently Shutdown, Estimated Startup Date: Notes:

CUMULATIVE UNIT CAPACITY FACTORS (MDC \& DER) ARE CALCULATED WITH WEIGHTED AVERAGES. 


\begin{tabular}{|c|c|c|c|c|c|c|c|c|c|}
\hline No. & Date & Type & Hours & Reason & Method & LER Number & System & Component & Cause and Corrective Action To Prevent Recurrence \\
\hline 9409 & $12 / 09 / 94$ & $\mathbf{F}$ & 241.6 & A & 2 & 94007 & CHS & $2 \mathrm{CHS}^{*} \mathrm{H}$ & $\begin{array}{l}\text { HIGH DRYWELLL LEAKAGE. HIGH PRESSURE CORE SPRAY } \\
\text { BLOCKING VALVE 2CSH*HCV } 120 \text { WAS REPACKED AND } \\
\text { BACKSEATED. }\end{array}$ \\
\hline 9410 & $12 / 21 / 94$ & $\mathbf{F}$ & 0.0 & A & 5 & & & & $\begin{array}{l}\text { REDUCED REACTOR POUER TO SUAP FEEDWATER PUMPS DUE } \\
\text { TO MALFUNCTION OF FEEDWATER FLOW CONTROL VALVE } \\
\text { 2FWS-LVIOB. }\end{array}$ \\
\hline
\end{tabular}

\begin{tabular}{l} 
TYPE \\
\hline F: Forced \\
S: Scheduled
\end{tabular}

\section{REASON}

A-Equipment Failure

B-Maintenance or Test

C-Refuel ing

D-Regulatory Restriction

E-Operator Training \&

License Examination

G-operational Erro

H-Other

\section{METHOO}

1-Manua

2-Manual Scram

3-Auto Scram

5-Reduced Load

9-0ther

\section{SYSTEM}

IEEE Standard

NUREG-0161 Exhibit
COMPONENT

IEEE Standard

MUREG-0161 Exhibit H 


\section{FACILITY DESCRIPTION}

\section{LOCATION}

STATE.................. NEW YORK

COUNTY.

OSWEGO

DIST AND DIRECTION FRON

NEAREST POPULATION CTR.

TYPE OF REACTOR

BUR

DATE INITIAL CRITICALITY...... MAY 23, 1987

DATE INITIAL ELECTRICITY...... AUGUST 08, 1987

DATE COMMERCIAL OPERATE....... APRIL 05, 1988

COMDENSER COOLING METHOD....... COOLING TONER

CONDENSER COOLING WATER....... LAKE ONTARIO

ELECTRIC RELIABILITY

COUNCIL................. NORTHEASTERN PONER COORDINATION COUNCIL.

\section{UTILITY \& CONTRACTOR INFORMATION}

\section{UTILITY}

LICENSEE.

CORPORATE ADDRESS

NIAGARA MOHAMK PONER CORP.

301 PLAINFIELD RD

SYRACUSE, NEH YORK 13212

\section{CONTRACTOR}

ARCHITECT/ENGINEER......... STOME \& WEBSTER

NUC STEAM SYS SUPPLIER..... GEMERAL ELECTRIC

CONSTRUCTOR.

STONE \& WEBSTER

TURBINE SUPPLIER........... GEMERAL ELECTRIC

\section{REGULATORY INFORMATION}

IE REGION RESPONSIBLE......... 1

IE RESIDENT INSPECTOR

BARRY MORRIS

LICENSING PROJ MAMAGER

DONALD S. BRINKMAN

DOCKET MUMBER.

$50-410$

LICENSE \& DATE ISSUANCE

MPF 069, JULY 02, 1987 
1. Docket: $50-338$

OPERATING STATUS

2. Reporting Period: DECEMBER 1994

Outage + On-Line Hrs:

744.0

3. Utility Contact: J. A. STALL (703) 894-2101

4. Licensed Thermal Power (MWt):

5. Nameplate Rating (Gross MWe):

6. Design Electrical Rating (Net MUe):

7. Maximum Dependable Capacity (Gross MHe):

8. Maximum Dependable Capacity (Net MHe):

9. If Changes Occurred Above Since Last Report, Give Reasons:

10. Power Level To thich Restricted, If Any (Net MWe):

11. Reasons for Restrictions, If Any:

\begin{tabular}{|c|c|c|c|}
\hline & MONTH & YEAR & CUMULATIVE \\
\hline 12. Report Period Hrs & 744.0 & $8,760.0$ & $144,876.0$ \\
\hline 13. Hours Reactor Critical & 744.0 & $8,042.1$ & $108,447.8$ \\
\hline 14. Rx Reserve Shtown Hrs & 0.0 & 103.7 & $6,930.5$ \\
\hline 15. Hrs Generator On-Line & 744.0 & $8,012.8$ & $105,453.7$ \\
\hline 16. Unit Reserve Shtchn Hrs & 0.0 & 0.0 & 0.0 \\
\hline 17. Gross Therm Ener (MUH) & $2,151,651.5$ & $21,733,342.6$ & $280,185,579.0$ \\
\hline 18. Gross Elec Ener (MUH) & $709,438.0$ & $7,160,263.0$ & $92,075,620.0$ \\
\hline 19. Net Elec Ener (MHH) & $675,974.0$ & $6,795,701.0$ & $87,202,286.0$ \\
\hline 20. Unit Service Factor & 100.0 & 91.5 & 72.8 \\
\hline 21. Unit Avail Factor & 100.0 & 91.5 & 72.8 \\
\hline 22. Unit Cap Factor (MDC Net) & 101.0 & 86.2 & 67.3 \\
\hline 23. Unit Cap Factor (DER Net) & 100.2 & 85.5 & 66.4 \\
\hline 24. Unit Forced Outage Rate & 0.0 & 0.0 & 10.0 \\
\hline 25. Forced Outage Hours & 0.0 & 0.0 & $11,720.7$ \\
\hline
\end{tabular}

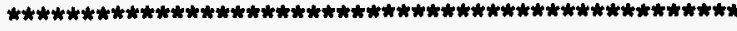 * NORTH ANNA 1}

AVERAGE DAILY POWER LEVEL (Net MWe)

\begin{tabular}{ll} 
DAY & PONER \\
\hline 1 & 910 \\
\hline 2 & 910 \\
\hline 3 & 910 \\
\hline 4 & 911 \\
\hline 5 & 910 \\
\hline 6 & 909 \\
\hline 7 & 911 \\
\hline 8 & 911 \\
\hline 9 & 900 \\
\hline 10 & 910 \\
\hline 11 & 910 \\
\hline 12 & 909 \\
\hline 13 & 909 \\
\hline 14 & 908 \\
\hline 15 & 909 \\
\hline
\end{tabular}

\begin{tabular}{ll} 
DAY & POMER \\
\hline 16 & 909 \\
\hline 17 & 908 \\
\hline 18 & 908 \\
\hline 19 & 908 \\
\hline 20 & 908 \\
\hline 21 & 909 \\
\hline 22 & 907 \\
\hline 23 & 908 \\
\hline 24 & 908 \\
\hline 25 & 909 \\
\hline 26 & 909 \\
\hline 27 & 909 \\
\hline 28 & 908 \\
\hline 29 & 908 \\
\hline 30 & 907 \\
\hline 31 & 907 \\
\hline
\end{tabular}

26. Shutdowns Scheduled Over Next Six Months (Type, Date, Duration):

27. If Currently Shutdown, Estimated Startup Date:

Notes:

CUMULATIVE UNIT CAPACITY FACTOR (MDC NET) IS CALCULATED WITH A WEIGHTED AVERAGE. 


No. Date Type Hours Reason Method LER Number System Component Cause and Corrective Action To Prevent Recurrence

\section{TYPE \\ F: Forced \\ S: Scheduled}

REASON

A-Equipment Failure

B-Maintenance or Test

C-Refuel ing

D-Regulatory Restriction

E-Operator Training \&

License Examination

F-Administrative

G-Operational Error

H-Other

\begin{tabular}{l} 
METHOD \\
\hline 1-Manual \\
2-Manual Scram \\
3-Auto Scram \\
4-Cont inued \\
5-Reduced Load \\
9-Other
\end{tabular}

\section{SYSTEM}

IEEE Standard

NUREG-0161 Exhibit $F$

\section{COMPONENT}

IEEE Standard

MUREG-0161 Exhibit H 


\section{FACILITY DESCRIPTION}

\section{LOCATION}

STATE.

countY.

VIRGIKIA

LOUISA

\section{DIST AND DIRECTION FRO}

MEAREST POPULATION CTR.... $40 \mathrm{MI}$ NH OF RICHMOND, VA

TYPE OF REACTOR

DATE INITIAL CRITICALITY...... APRIL 05, 1978

DATE INITIAL ELECTRICITY...... APRIL 17, 1978

DATE COMARCIAL OPERATE....... JUME 06, 1978

CONDENSER COOLING METHOD...... ONCE THRU

CONDENSER COOLING WATER....... LAKE ANNA

ELECTRIC RELIABILITY

COUNCIL................... SOUTHEASTERN ELECTRIC RELIABILITY COUNCIL

\section{UTILITY \& CONTRACTOR INFORMATION}

UTILITY

LICENSEE................. VIRGINIA ElECTRIC \& POWER CO.

CORPORATE ADDRESS.......... P.0. BOX 26666

RICHMOND, VIRGINIA 23261

\section{CONTRACTOR}

ARCHITECT/ENGINEER......... STONE \& MeBSTER

MUC STEAM SYS SUPPLIER..... . WESTINGHOUSE

CONSTRUCTOR............... STONE \& MEBSTER

TURBINE SUPPLIER........... WESTIMGHOUSE

\section{REGULATORY IMFORMATION}

IE REGION RESPOWSIBLE......... 2

IE RESIDENT INSPECTOR

RICHARD MCWHORTER

LICENSING PROJ MANAGER.

LEON B. ENGLE

DOCKET NUMBER.

$50-338$

LICENSE \& DATE ISSUANCE

MPF 004, APRIL 01, 1978 
1. Docket: $50-339$

OPERATING STATUS

2. Reporting Period: DECEMBER 1994

Outage + On-Line Hrs: $\mathbf{7 4 4 . 0}$

3. Utility Contact: J. A. STALL (703) 894-2101

4. Licensed Thermal Power (MWt):

\begin{tabular}{c}
\hline 2893 \\
\hline 979 \\
\hline 907 \\
\hline 935 \\
\hline 887
\end{tabular}

5. Nameplate Rating (Gross MWe):

6. Design Electrical Rating (Net MWe):

7. Maximn Dependable Capacity (Gross MHe):

8. Maximum Dependable Capacity (Net MHe):

9. If Changes Occurred Above Since Last Report, Give Reasons:

10. Power Level To which Restricted, If Any (Net MWe):

11. Reasons For Restrictions, If Any:

\begin{tabular}{|c|c|c|c|}
\hline & MONTH & YEAR & CUMULATIVE \\
\hline 12. Report Period Hrs & 744.0 & $8,760.0$ & $123,144.0$ \\
\hline 13. Hours Reactor Critical & 744.0 & $8,559.9$ & $102,933.4$ \\
\hline 14. Rx Reserve Shtdwn Hrs & 0.0 & 95.7 & $6,508.9$ \\
\hline 15. Hrs Generator On-Line & 744.0 & $8,518.3$ & $101,835.7$ \\
\hline 6. Unit Reserve Shtdwn Hrs & 0.0 & 0.0 & 0.0 \\
\hline 17. Gross Therm Ener (MUH) & $2,151,057.9$ & $24,390,305.7$ & $276,615,963.4$ \\
\hline 3. Gross Elec Ener (MUH) & $695,801.0$ & $7,881,381.0$ & $90,476,857.0$ \\
\hline 19. Net Elec Ener (MWH) & $662,029.0$ & $7,490,267.0$ & $86,539,968.0$ \\
\hline 20. Unit Service Factor & 100.0 & 97.2 & 82.7 \\
\hline 21. Unit Avail Factor & 100.0 & 97.2 & 82.7 \\
\hline 22. Unit Cap Factor (MDC Net) & 100.3 & 96.4 & 78.1 \\
\hline 23. Unit Cap Factor (DER Net) & 98.1 & 94.3 & 77.5 \\
\hline 24. Unit Forced Outage Rate & 0.0 & 2.8 & 5.4 \\
\hline 5. Forced Outage Hours & 0.0 & 241.7 & $5,793.4$ \\
\hline
\end{tabular}

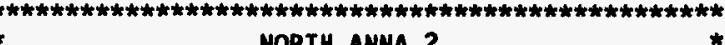

MORTH ANMA 2

AVERAGE DAILY POMER LEVEL (Net MWe)

\begin{tabular}{lc} 
DAY & POWER \\
1 & 891 \\
\hline 2 & 881 \\
\hline 3 & 888 \\
\hline 4 & 889 \\
\hline 5 & 891 \\
\hline 6 & 890 \\
\hline 7 & 891 \\
\hline 8 & 890 \\
\hline 9 & 891 \\
\hline 10 & 890 \\
\hline 11 & 891 \\
\hline 12 & 891 \\
\hline 13 & 891 \\
\hline 14 & 891 \\
\hline 15 & 891 \\
\hline
\end{tabular}

\begin{tabular}{ll} 
DAY & POWER \\
\hline 16 & 891 \\
\hline 17 & 890 \\
\hline 18 & 891 \\
\hline 19 & 891 \\
\hline 20 & 890 \\
\hline 21 & 891 \\
\hline 22 & 891 \\
\hline 23 & 890 \\
\hline 24 & 891 \\
\hline 25 & 891 \\
\hline 26 & 891 \\
\hline 27 & 891 \\
\hline 28 & 890 \\
\hline 29 & 890 \\
\hline 30 & 883 \\
\hline 31 & 887 \\
\hline
\end{tabular}

26. Shutdowns Scheduled Over Next Six Months (Type, Date, Duration): REFUELING/STEAM GENERATOR REPLACEMENT OUTAGE, MARCH 25, 1995, 105 DAYS.

27. If Currently Shutdown, Estimated Startup Date:

Notes:

CUMULATIVE UNIT CAPACITY FACTOR (MDC NET) IS CALCULATED WITH A MEIGHTED AVERAGE. 


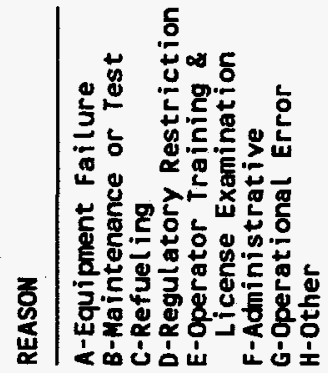

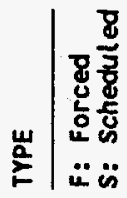




\section{FACILITY DESCRIPTION}

\section{LOCATION}

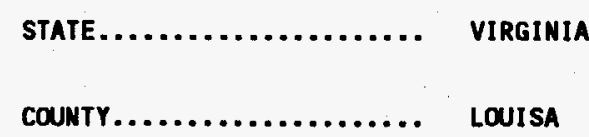

\section{DIST AND DIRECTION FRO}

MEAREST POPULATION CTR..... 40 MI NH OF RICHMOND, VA

TYPE OF REACTOR............ PWR

DATE IMITIAL CRITICALITY...... JUNe 12, 1980

DATE INITIAL ELECTRICITY...... AUGUST 25, 1980

date Commercial operate....... DeCember 14, 1980

CONDENSER COOLING METHOD...... ONCE THRU

CONDENSER COOLING WATER....... LAKE AMMA

ELECTRIC RELIABILITY

COUNCIL.................. SOUTHEASTERN ELECTRIC
UTILITY \& CONTRACTOR INFORMATION

\section{UTILITY}

licensee................ virgimia electric \& PONER co.

CORPORATE ADDRESS......... P.O. BOX 26666

RICHMOND, VIRGINIA 23261

\section{CONTRACTOR}

ARCHITECT/ENGINEER.

STONE \& WEBSTER

NUC STEAM SYS SUPPLIER

WEST INGHOUSE

CONSTRUCTOR

STONE \& WEBSTER

TURBINE SUPPLIER

WESTINGHOUSE

\section{REGULATORY INFORMATION}

IE REGION RESPONSIBLE........ 2

IE RESIDENT INSPECTOR.......... RICHARO MCUHORTER

LICENSING PROJ MANAGER......... LEON B. ENGLE

DOCKET MUMBER.............. 50-339

LICENSE \& DATE ISSUANCE........ NPF 007, AUGUST 21, 1980 
1. Docket: 50-269

OPERATING STATUS

2. Reporting Period: DECEMBER 1994

Outage + On-Line Hrs: $\mathbf{7 4 4 . 0}$

3. Utility Contact: R. A. WILLIAMS (704) 382-5346

4. Licensed Thermal Power (MWt):

5. Nameplate Rating (Gross MWe):

6. Design Electrical Rating (Net MWe):

7. Maximm Dependable Capacity (Gross MHe):

8. Maximum Dependable Capacity (Net MWe):

9. If Changes Occurred Above Since Last Report, Give Reasons:

10. Power Level To Which Restricted, If Any (Net MWe):

11. Reasons For Restrictions, If Any:

\begin{tabular}{|c|c|c|c|}
\hline & MONTH & YEAR & CUMULATIVE \\
\hline 12. Report Period Hrs & 744.0 & $8,760.0$ & $188,137.0$ \\
\hline 13. Hours Reactor Critical & 744.0 & $7,371.5$ & $146,094.3$ \\
\hline 14. Rx Reserve Shtdwn Hrs & 0.0 & 0.0 & 0.0 \\
\hline 15. Hrs Generator On-Line & 744.0 & $7,302.9$ & $143,344.7$ \\
\hline 16. Unit Reserve Shtown Hrs & 0.0 & 0.0 & 0.0 \\
\hline 17. Gross Therm Ener (MUH) & $1,913,064.0$ & $18,567,888.0$ & $352,164,070.0$ \\
\hline 18. Gross Elec Ener (MUH) & $662,002.0$ & $6,384,190.0$ & $121,752,963.0$ \\
\hline 19. Net Elec Ener (MUH) & $633,558.0$ & $6,086,245.0$ & $115,692,609.0$ \\
\hline 20. Unit Service Factor & 100.0 & 83.4 & 76.2 \\
\hline 21. Unit Avail Factor & 100.0 & 83.4 & 76.2 \\
\hline 22. Unit Cap Factor (MDC Net) & 100.7 & 82.1 & 71.8 \\
\hline 23. Unit Cap Factor (DER Net) & 96.1 & 78.4 & 69.4 \\
\hline 24. Unit Forced Outage Rate & 0.0 & 0.5 & 9.9 \\
\hline 25. Forced Outage Hours & 0.0 & 35.2 & $15,817.5$ \\
\hline
\end{tabular}

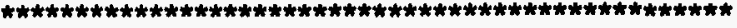
OCONEE 1

\section{*}

AVERAGE DAILY POWER LEVEL (Net MHe)

\begin{tabular}{|c|c|c|c|}
\hline DAY & PONER & DAY & PONER \\
\hline 1 & 851 & 16 & 851 \\
\hline 2 & 851 & 17 & 851 \\
\hline 3 & 851 & 18 & 852 \\
\hline 4 & 851 & 19 & 851 \\
\hline 5 & 852 & 20 & 851 \\
\hline 6 & 852 & 21 & 852 \\
\hline 7 & 852 & 22 & 852 \\
\hline 8 & 851 & 23 & 852 \\
\hline 9 & 848 & 24 & 852 \\
\hline 10 & 850 & 25 & 852 \\
\hline 11 & 851 & 26 & 852 \\
\hline 12 & 851 & 27 & 852 \\
\hline 13 & 851 & 28 & 852 \\
\hline 14 & 852 & 29 & 853 \\
\hline 15 & 851 & 30 & 853 \\
\hline & & 31 & 853 \\
\hline
\end{tabular}

26. Shutdowns Scheduled Over Next Six Months (Type, Date, Duration):

27. If Currently Shutdown, Est imated Startup Date:

Notes:

CUMULATIVE UNIT CAPACITY FACTOR (MDC MET) IS CALCULATED HITH A WEIGHTED AVERAGE. 
TYPE

F: Forced

s: Scheduled

\section{REASON}

A-Equipment failure

B-Maintenance or Test

C-Refuel ing

D-Regulatory Restriction

E-Operator Training \&

License Examination

F-Administrative

G-Operational Error

H-Other
METHOD

1-Manual

2-Manual Scram

3-Auto Scram

4-Cont inued

5-Reduced Load

9-0ther

\section{SYSTEM}

IEEE Standard

805-1984 and/or

NUREG-0161 Exhibit F
COMPONENT

IEEE Standard

803A-1983 and/or

NUREG-0161 Exhibit 


\section{FACILITY DESCRIPTION}

LOCATION

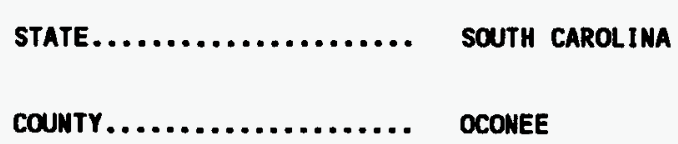

DIST AND DIRECTION FRON

TYPE OF REACTOR.

PWR

DATE IMITIAL CRITICALITY...... APRIL 19, 1973

DATE INITIAL ELECTRICITY...... MAY 06, 1973

DATE COMMERCIAL OPERATE....... JULY 15, 1973

CONDENSER COOLING METHOD...... ONCE THRU

CONDENSER COOLING MATER....... LAKE KEONEE

ELECTRIC RELIABILITY

COUNCIL.................. SOUTHEASTERN ELECTRIC RELIABILITY COUNCIL

\section{UTILITY \& CONTRACTOR INFORMATION}

\section{UTILITY}

LICENSEE.

CORPORATE ADORESS

DUKE PONER CO.

422 SOUTH CHURCH STREET

CHARLOTTE, NORTH CAROLIMA 28242

\section{CONTRACTOR}

ARCHITECT/ENGINEER.......... DUKE \& BECHTEL

NUC STEAM SYS SUPPLIER...... BABCOCK \& HILCOX

CONSTRUCTOR

DUKE PONER

TURBINE SUPPLIER.

GEMERAL ELECTRIC

\section{REGULATORY INFORMATION}

IE REGION RESPONSIBLE.......... 2

JE RESIDENT INSPECTOR........... PAUL. HARMON

LICENSING PROJ MANAGER........ LEONARD A. WIENS

DOCKET MUMBER.............. 50-269

LICENSE \& DATE ISSUAMCE........ DPR 038, FEBRUARY 06, 1973 
1. Docket: $50-270$

OPERATING STATUS

2. Reporting Period: DECEMBER 1994

Outage + On-Line Hrs:

744.0

3. Utility Contact: R. A. WILLIAMS (704) 382-5346

4. Licensed Thermal Power (MUt):

5. Nameplate Rating (Gross MWe):

6. Design Electrical Rating (Net MHe):

7. Naximm Dependable Capacity (Gross MHe):

8. Maximum Dependable Capacity (Net MHe):

$\begin{array}{r}\hline 2568 \\ \hline 934 \\ \hline 886 \\ \hline 886 \\ \hline 846 \\ \hline\end{array}$

9. If Changes Occurred Above Since Last Report, Give Reasons:

10. Power Level To Which Restricted, If Any (Net MWe):

11. Reasons For Restrictions, if Any:

\begin{tabular}{|c|c|c|c|}
\hline 12. Report Period Mrs & $\begin{array}{l}\text { MONTH } \\
744.0\end{array}$ & $\begin{array}{l}\text { YEAR } \\
8,760.0\end{array}$ & $\begin{array}{l}\text { CUMULATIVE } \\
178,057.0\end{array}$ \\
\hline 13. Hours Reactor Critical & 723.4 & $7,387.2$ & $141,384.0$ \\
\hline 14. Rx Reserve Shtdwn Hrs & 0.0 & 0.0 & 0.0 \\
\hline 15. Hrs Generator On-Line & 720.5 & $7,295.6$ & $139,432.5$ \\
\hline 16. Unit Reserve Shtdwn Hrs & 0.0 & 0.0 & 0.0 \\
\hline 17. Gross Therm Ener (MWH) & $1,837,248.0$ & $18,586,992.0$ & $340,003,574.0$ \\
\hline 18. Gross Elec Ener (MWH) & $637,761.0$ & $6,450,110.0$ & $116,327,658.0$ \\
\hline 19. Net Elec Ener (MUH) & $609,522.0$ & $6,148,500.0$ & $110,767,570.0$ \\
\hline 20. Unit Service Factor & 96.8 & 83.3 & 78.3 \\
\hline 21. Unit Avail Factor & 96.8 & 83.3 & 78.3 \\
\hline 22. Unit Cap Factor (MDC Net) & 96.8 & 83.0 & 72.5 \\
\hline 23. Unit Cap Factor (DER Net) & 92.5 & 79.2 & 70.2 \\
\hline 24. Unit Forced Outage Rate & 3.2 & 5.6 & 8.6 \\
\hline 25. Forced Outage Hours & 23.5 & 430.0 & $13,197.8$ \\
\hline
\end{tabular}

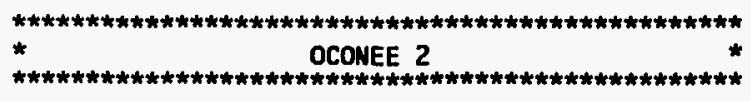

AVERAGE DAILY POWER LEVEL (Het MHe)

\begin{tabular}{ll} 
DAY & POWER \\
\hline & 860 \\
\hline 2 & 859 \\
\hline 3 & 859 \\
\hline 4 & 859 \\
\hline 5 & 859 \\
\hline 6 & 859 \\
\hline 7 & 859 \\
\hline 8 & 501 \\
\hline 9 & 139 \\
\hline 10 & 834 \\
\hline 11 & 856 \\
\hline 12 & 856 \\
\hline 13 & 856 \\
\hline 14 & 855 \\
\hline 15 & 855 \\
\hline
\end{tabular}

\begin{tabular}{|cc} 
DAY & PONER \\
\hline 16 & 855 \\
\hline 17 & 855 \\
\hline 18 & 855 \\
\hline 19 & 854 \\
\hline 20 & 854 \\
\hline 21 & 854 \\
\hline 22 & 854 \\
\hline 23 & 854 \\
\hline 24 & 854 \\
\hline 25 & 854 \\
\hline 26 & 854 \\
\hline 27 & 853 \\
\hline 28 & 854 \\
\hline 29 & 854 \\
\hline 30 & 853 \\
\hline 31 & 820 \\
\hline
\end{tabular}

26. Shutdowns Scheduled Over Next Six Months (Type, Date, Duration):

27. If Currently Shutdown, Estimated Startup Date:

Notes:

CUMULATIVE UNIT CAPACITY FACTOR (MDC NET) IS CALCULATED WITH A WEIGHTED AVERAGE. 


\begin{tabular}{|c|c|c|c|c|c|c|c|c|c|}
\hline No. & Date & Type & Hours & Reason & Method & LER Number & System & Component & Cause and Corrective Action To Prevent Recurrence \\
\hline 8 & $12 / 08 / 94$ & $\mathbf{F}$ & 23.5 & A & 3 & & EC & CKTBKR & $\begin{array}{l}\text { REACTOR/TURBINE TRIP DUE TO LOSS OF '2KI' } \\
\text { BREAKER. }\end{array}$ \\
\hline $11-p$ & $12 / 09 / 94$ & $\mathbf{F}$ & 0.0 & A & 9 & & HB & HTEXCH & $\begin{array}{l}\text { POWER HOLD TO IHVESTIGATE ' } 2 A \text { ' MOISTURE SEPARATOR } \\
\text { REHEATER PROBLEMS. }\end{array}$ \\
\hline 12-P & $12 / 09 / 94$ & $\mathbf{F}$ & 0.0 & B & 9 & & IA & INSTRU & $\begin{array}{l}\text { PONER HOLD FOR NUCLEAR INSTRUMENTATION } \\
\text { CALIBRATION CHECK. }\end{array}$ \\
\hline
\end{tabular}

\begin{tabular}{|c|c|c|c|c|}
\hline TYPE & REASON & METHOD & SYSTEM & COMPONENT \\
\hline $\begin{array}{l}\text { F: Forced } \\
\text { s: Scheduled }\end{array}$ & $\begin{array}{l}\text { A-Equipment Failure } \\
\text { B-Maintenance or Test } \\
\text { C-Refuel ing } \\
\text { D-Regulatory Restriction } \\
\text { E-Operator Training \& } \\
\text { License Examination } \\
\text { F-Administrative } \\
\text { G-Operat ional Error } \\
\text { H-Other }\end{array}$ & $\begin{array}{l}\text { 1-Manual } \\
\text { 2-Manual Scram } \\
\text { 3-Auto Scram } \\
\text { 4-Continued } \\
\text { 5-Reduced Load } \\
\text { 9-Other }\end{array}$ & $\begin{array}{l}\text { IEEE Standard } \\
805-1984 \text { and/or } \\
\text { NUREG-0161 Exhibit F }\end{array}$ & $\begin{array}{l}\text { IEEE Standard } \\
803 A-1983 \text { and/or } \\
\text { MUREG-0161 Exh ibit H }\end{array}$ \\
\hline
\end{tabular}




\section{FACILITY DESCRIPTION}

LOCATION

STATE.

COUNTY.

SOUTH CAROLIHA

OCONEE

\section{DIST AND DIRECTION FROM}

MEAREST POPULATION CTR.

TYPE OF REACTOR

30 MI W OF GREENVILLE, SC

\section{PUR}

DATE INITIAL CRITICALITY...... NOVEMBER 11, 1973

DATE INITIAL ELECTRICITY...... DECEMBER 05, 1973

DATE COMmercial OPERATE....... SEPTEMBER 09, 1974

CONDENSER COOLING METHOD...... ONCE THRU

CONDENSER COOLING WATER....... LAKE KEOMEE

ELECTRIC RELIABILITY

COUNCIL................. SOUTHEASTERM ELECTRIC

RELIABILITY COUNCIL

\section{UTILITY \& CONTRACTOR INFORMATION}

UTILITY

LICENSEE

CORPORATE ADDRESS

DUKE POWER CO.

422 SOUTH CHURCH STREET

CHARLOTTE, NORTH CAROLIMA 28242

\section{CONTRACTOR}

ARCHI TECT/ENGINEER.

DUKE \& BECHTEL

NUC STEAM SYS SUPPLIER.

BABCOCK \& NILCOX

CONSTRUCTOR.

DUKE POWER

TURBINE SUPPLIER

Gemeral electric

REGULATORY INFORMATION

IE REGION RESPONSIBLE...........

IE RESIDENT INSPECTOR.......... PAUL HARMON

LICENSING PROJ MANAGER......... LEONARD A. WIENS

DOCKET NUMBER.............. 50-270

LICENSE \& DATE ISSUANCE........ DPR 047, OCTOBER 06, 1973 
1. Docket: $50-287$

OPERATING STATUS

2. Reporting Period: DECEMBER 1994

Outage + On-Line Hrs:

744.0

3. Utility Contact: R. A. WILLIAMS (704) 382-5346

4. Licensed Thermal Power (mit):

5. Nameplate Rating (Gross MWe):

6. Design Electrical Rating (Net MWe):

7. Maximum Dependable Capacity (Gross MWe):

8. Maximum Dependable Capacity (Net MWe):

9. If Changes Occurred Above Since Last Report, Give Reasons:

10. Power Level To thich Restricted, If Any (Net MWe):

11. Reasons For Restrictions, If Any:

\begin{tabular}{|c|c|c|c|}
\hline & MONTH & YEAR & CUNULATIVE \\
\hline 12. Report Period Hrs & 744.0 & $8,760.0$ & $175,704.0$ \\
\hline 13. Hours Reactor Critical & 744.0 & $6,835.7$ & $136,026.8$ \\
\hline 14. RX Reserve Shtdwn Hrs & 0.0 & 0.0 & 0.0 \\
\hline 15. Hrs Generator on-Line & 744.0 & $6,782.4$ & $134,236.7$ \\
\hline 16. Unit Reserve Shtdwn Hrs & 0.0 & 0.0 & 0.0 \\
\hline 17. Gross Therm Ener (MWH) & $1,915,512.0$ & $17,194,080.0$ & $333,533,769.0$ \\
\hline 18. Gross Elec Ener (MWH) & $665,409.0$ & $5,949,505.0$ & $115,069,765.0$ \\
\hline 19. Net Elec Ener (MUH) & $637,444.0$ & $5,668,567.0$ & $109,746,337.0$ \\
\hline 20. Unit Service Factor & 100.0 & 77.4 & 76.4 \\
\hline 21. Unit Avail Factor & 100.0 & 77.4 & 76.4 \\
\hline 22. Unit Cap Factor (MDC Net) & 101.3 & 76.5 & 73.9 \\
\hline 23. Unit Cap Factor (DER Net) & 96.7 & 73.0 & 70.5 \\
\hline 24. Unit Forced Outage Rate & 0.0 & 8.4 & 10.2 \\
\hline 25. Forced Outage Hours & 0.0 & 620.7 & $15,315.1$ \\
\hline
\end{tabular}

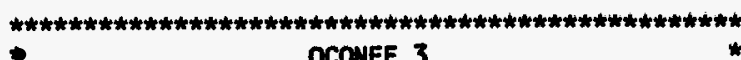

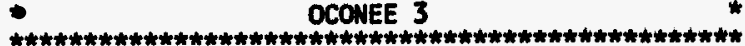

AVERAGe DAILY POWER LeVEL (Net MUe)

\begin{tabular}{ll} 
DAY & PONER \\
\hline & 856 \\
\hline 2 & 857 \\
\hline 3 & 857 \\
\hline 4 & 857 \\
\hline 5 & 857 \\
\hline 6 & 857 \\
\hline 7 & 857 \\
\hline 8 & 857 \\
\hline 9 & 857 \\
\hline 10 & 857 \\
\hline 11 & 857 \\
\hline 12 & 857 \\
\hline 13 & 857 \\
\hline 14 & 857 \\
\hline 15 & 857 \\
\hline
\end{tabular}

\begin{tabular}{cc} 
DAY & PONER \\
\hline 16 & 857 \\
\hline 17 & 857 \\
\hline 18 & 857 \\
\hline 19 & 858 \\
\hline 20 & 858 \\
\hline 21 & 858 \\
\hline 22 & 858 \\
\hline 23 & 858 \\
\hline 24 & 858 \\
\hline 25 & 858 \\
\hline 26 & 858 \\
\hline 27 & 858 \\
\hline 28 & 858 \\
\hline 29 & 858 \\
\hline 30 & 858 \\
\hline 31 & 838 \\
\hline
\end{tabular}

26. Shutdowns Scheduled Over Mext six Months (Type, Date, Duration): REFUELING OUtAGe, JUME 8, 1995, 45 DAYS.

27. If Currently Shutdown, Estimated Startup Date:

Notes:

CUMULATIVE UMIT CAPACITY FACTOR (MDC NET) IS CALCULATED MITH A MEIGHTED AVERAGE. 


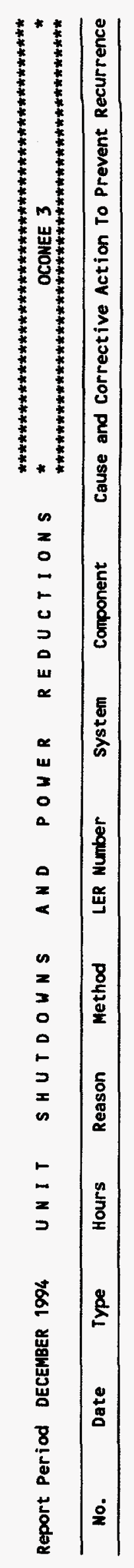

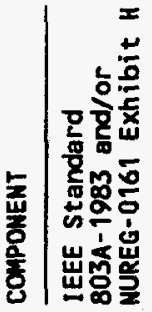

|

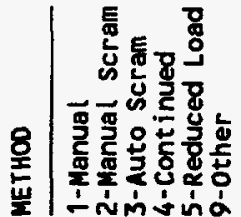

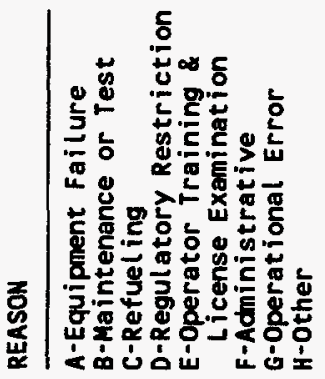

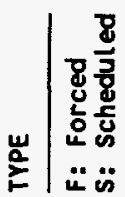




\section{LOCATION}

STATE.

countr.

SOUTH CAROLINA

\section{DIST AND DIRECTION FROM}

MEAREST POPULATION CTR

TYPE OF REACTOR

30 MI $W$ OF GREENVILLE, SC

PUR

DATE INITIAL CRITICALITY

DATE IMITIAL ELECTRICITY.

OATE COMMERCIAL OPERATE.

CONDENSER COOLING METHOD.

CONDENSER COOLING WATER....... LAKE KEOMEE

ELECTRIC RELIABILITY

COUHCIL RELIABILITY RELIABILITY COUNCIL
UTILITY

LICENSEE.

CORPORATE ADDRESS

\section{CONTRACTOR}

ARCHITECT/ENGINEER

NUC STEAM SYS SUPPLIER...... BABCOCK \& HILCOX

CONSTRUCTOR

DUKE PONER

TURBINE SUPPLIER

GEMERAL ELECTRIC

REGULATORY INFORMATION

IE REGION RESPONSIBLE.........

IE RESIDENT INSPECTOR.......... PAUL HARMON

LICENSING PROJ MANAGER......... LEONARD A. WIENS

DOCKET MUMBER.............. 50-287

LICENSE \& DATE ISSUANCE........ DPR 055, JULY 19, 1974 
1. Docket: $50-219$

OPERATING STATUS

2. Reporting Period: DECEMBER 1994

Outage + On-Line Hrs:

744.0

3. Utility Contact: PAUL G. EDELMANN (609) 971-4097

4. Licensed Thermal Power (MWt):

5. Nameplate Rating (Gross MHe):

6. Design Electrical Rating (Net MHe):

7. Maximum Dependable Capacity (Gross MWe):

8. Maximum Dependable Capacity (Net MHe):

\begin{tabular}{c}
1930 \\
\hline 550 \\
\hline 650 \\
\hline 641 \\
\hline 619
\end{tabular}

9. If Changes Occurred Above Since Last Report, Give Reasons:

MAXIMUM DEPENDABLE CAPACITIES GROSS AND NET INCREASED DUE TO TURBINE UPGRADE.

10. Power Level To thich Restricted, If Any (Net MWe):

11. Reasons For Restrictions, If Any:

\begin{tabular}{|c|c|c|c|}
\hline & MONTH & YEAR & CUMULATIVE \\
\hline 12. Report Period Hrs & 744.0 & $8,760.0$ & $219,360.0$ \\
\hline 13. Hours Reactor Critical & 425.7 & $6,201.6$ & $145,800.8$ \\
\hline 14. Rx Reserve Shtdin Hrs & 0.0 & 0.0 & 918.2 \\
\hline 15. Hrs Generator On-Line & 380.0 & $6,097.3$ & $142,299.0$ \\
\hline 16. Unit Reserve Shtdwn Hrs & 0.0 & 0.0 & $1,208.6$ \\
\hline 17. Gross Therm Ener (MUH) & $625,075.0$ & $11,410,327.0$ & $244,483,586.0$ \\
\hline 18. Gross Elec Ener (MUH) & $210,475.0$ & $3,781,977.0$ & $82,026,291.0$ \\
\hline 19. Net Elec Ener (MuH) & $200,103.0$ & $3,627,373.0$ & $78,726,979.0$ \\
\hline 20. Unit Service Factor & 51.1 & 69.6 & 64.9 \\
\hline 21. Unit Avail Factor & 51.1 & 69.6 & 65.4 \\
\hline 22. Unit Cap Factor (MDC Net) & 43.8 & 67.8 & 58.5 \\
\hline 23. Unit Cap Factor (DER Net) & 41.4 & 63.7 & 55.2 \\
\hline 24. Unit Forced Dutage Rate & 0.0 & 5.2 & 10.3 \\
\hline 25. Forced Outage Hours & 0.0 & 332.5 & $16,289.9$ \\
\hline
\end{tabular}

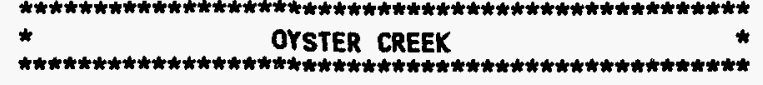

AVERAGE DAILY PONER LEVEL (Net MUe)

\begin{tabular}{cc} 
DAY & PONER \\
\hline 1 & 0 \\
\hline 3 & 0 \\
\hline 4 & 0 \\
\hline 5 & 0 \\
\hline 6 & 0 \\
\hline 7 & 0 \\
\hline 8 & 0 \\
\hline 9 & 0 \\
\hline 10 & 0 \\
\hline 11 & 0 \\
\hline 12 & 0 \\
\hline 13 & 0 \\
\hline 14 & 0 \\
\hline 15 & 0 \\
\hline
\end{tabular}

\begin{tabular}{cc} 
DAY & PONER \\
\hline 16 & 107 \\
\hline 17 & 278 \\
\hline 18 & 223 \\
\hline 19 & 287 \\
\hline 20 & 509 \\
\hline 21 & 636 \\
\hline 22 & 638 \\
\hline 23 & 642 \\
\hline 24 & 640 \\
\hline 25 & 640 \\
\hline 26 & 640 \\
\hline 27 & 640 \\
\hline 28 & 639 \\
\hline 29 & 638 \\
\hline 30 & 637 \\
\hline 31 & 637 \\
\hline
\end{tabular}

26. Shutdouns Scheduled Over Mext six Months (Type, Date, Duration):

27. If Currently Shutdown, Estimated Startup Date:

Notes:

MONTHLY, YTD, AND CUMULATIVE UNIT CAPACITY FACTORS (MOC NET) ARE CALCULATED WITH WEIGHTED AVERAGES. 
Report Period DECEMBER 1994

\begin{tabular}{|c|c|c|c|c|c|c|c|c|c|}
\hline No. & Date & Type & Hours & Reason & Method & LER Number & System & Component & Cause and Corrective Action To Prevent Recurrence \\
\hline
\end{tabular}

TYPE

F: Forced

F: Forced
S: Schedul ed

\begin{tabular}{l} 
REASON \\
\hline A-Equipment Failure \\
B-Maintenance or Test \\
C-Refuel ing \\
D-Regulatory Restriction \\
E-Operator Training \& \\
License Examination \\
F-Administrative \\
G-Operational Error \\
H-Other
\end{tabular}

METHOD

1-Manual

2-Manual Scram

3-Auto Scram

4-Cont inued

5-Reduced Load

9-0ther

G-Operationat Error

$H$-other

RC FUELXX REFUELING OUTAGE CONTINUED.

\begin{tabular}{lll} 
SYSTEM & & COMPONENT \\
\cline { 1 - 1 } IEEE Standard & & IEEE Standard \\
$805-1984$ and/or & & $803 A-1983$ and/or \\
NUREG-0161 Exhibit F & NUREG-0161 Exhib
\end{tabular}

805-1984 and/or $\quad 803 A-1983$ and/or 


\section{FACILITY DESCRIPTION}

LOCATION

STATE.

COUNTY

NEW JERSEY

OCEAN

\section{DIST AND DIRECTION FROM}

MEAREST POPULATIOH CTR

9 MI S OF TOMS RIVER, NJ

TYPE OF REACTOR

BUR

DATE INITIAL CRITICALITY...... MAY 03, 1969

DATE INITIAL ELECTRICITY...... SEPTEMBER 23, 1969

DATE COMMERCIAL OPERATE....... DECEMBER 01, 1969

CONDENSER COOLING METHOD...... ONCE THRU

CONDENSER COOLING WATER....... BARNEGAT BAY

\section{ELECTRIC RELIABILITY}

COUNCIL.......... MID-ATLANTIC AREA COUNCIL

\section{UTILITY \& CONTRACTOR INFORMATION}

\section{UTILITY}

LICENSEE. GPU NUCLEAR CORP.

CORPORATE ADDRESS

100 INTERPACE PARKWAY PARSIPPANY, NEH JERSEY 07054

CONTRACTOR

ARCHITECT/ENGIMEER......... BURNS \& ROE

NUC STEAM SYS SUPPLIER...... GENERAL ELECTRIC

CONSTRUCTOR.............. BURNS \& ROE

TURBINE SUPPLIER.......... GENERAL ELECTRIC

\section{REGULATORY IMFORMATION}

IE REGION RESPONSIBLE........ 1

IE RESIDENT INSPECTOR......... LARRY BRIGGS

LICENSING PROJ MANAGER. ALEXANDER W. DROMERICK

DOCKET MUMBER 50-219

LICENSE \& DATE ISSUANCE. DPR 016, AUGUST 01, 1969 
1. Docket: $\mathbf{5 0 - 2 5 5}$

OPERATING STATUS

2. Reporting Period: DECEMBER 1994

Outage + On-Line Hrs: $\mathbf{7 4 4 . 0}$

3. Utility Contact: B. H. BERLES (616) 764-8913 EXT 0190

4. Licensed Thermal Power (MHt):

5. Nameplate Rating (Gross MHe):

6. Design Electrical Rating (Net MWe):

7. Maximum Dependable Capacity (Gross MWe):

8. Maximum Dependable Capacity (Net MWe):

9. If Changes Occurred Above Since Last Report, Give Reasons:

10. Power Level To thich Restricted, If Any (Net MWe):

11. Reasons For Restrictions, If Any:

\begin{tabular}{c}
2530 \\
\hline 812 \\
\hline 805 \\
\hline 770 \\
\hline 730 \\
\hline
\end{tabular}

\begin{tabular}{|c|c|c|c|}
\hline & MONTH & YEAR & CUMULATIVE \\
\hline 12. Report Period Hrs & 744.0 & $8,760.0$ & $201,927.0$ \\
\hline 13. Hours Reactor Critical & 744.0 & $5,872.0$ & $114,312.0$ \\
\hline 14. Rx Reserve Shtdun Hrs & 0.0 & 0.0 & 0.0 \\
\hline 15. Hrs Generator On-Line & 744.0 & $5,860.5$ & $109,662.1$ \\
\hline 16. Unit Reserve Shtdm Hrs & 0.0 & 0.0 & 0.0 \\
\hline 17. Gross Therm Ener (MUH) & $1,867,368.0$ & $14,627,832.0$ & $238,290,651.0$ \\
\hline 18. Gross Elec Ener (MHH) & $611,208.0$ & $4,746,288.0$ & $\pi, 364,741.0$ \\
\hline 19. Het Elec Ener (MUH) & $581,734.0$ & $4,513,826.0$ & $71,096,511.0$ \\
\hline 20. Unit Service Factor & 100.0 & 66.9 & 54.3 \\
\hline 21. Unit Avail Factor & 100.0 & 66.9 & 54.3 \\
\hline 22. Unit Cap Factor (MDC Net) & 107.1 & 70.6 & 52.2 \\
\hline 23. Unit Cap Factor (DER Net) & 97.1 & 64.0 & 43.7 \\
\hline 24. Unit Forced Outage Rate & 0.0 & 33.1 & 29.9 \\
\hline 25. Forced Outage Hours & 0.0 & 2899.5 & $46,695.8$ \\
\hline
\end{tabular}

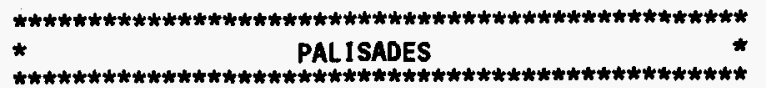

AVERAGE DAILY POWER LEVEL (Net MHe)

\begin{tabular}{|c|c|c|c|}
\hline DAY & POWER & DAY & PONER \\
\hline 1 & 786 & 16 & 778 \\
\hline 2 & 786 & 17 & 783 \\
\hline 3 & 786 & 18 & 779 \\
\hline 4 & 785 & 19 & 787 \\
\hline 5 & 785 & 20 & 779 \\
\hline 6 & 786 & 21 & 779 \\
\hline 7 & 787 & 22 & 779 \\
\hline 8 & 794 & 23 & 787 \\
\hline 9 & 780 & 24 & 780 \\
\hline 10 & 782 & 25 & 779 \\
\hline 11 & 792 & 26 & 783 \\
\hline 12 & 789 & 27 & 784 \\
\hline 13 & 790 & 28 & 784 \\
\hline 14 & 768 & 29 & 787 \\
\hline \multirow[t]{2}{*}{15} & 725 & 30 & 786 \\
\hline & & 31 & 784 \\
\hline
\end{tabular}

26. Shutdowns Scheduled Over Mext Six Months (Type, Date, Duration): REFUELING OUTAGE, MAY 20, 1995, 100 DAYS.

27. If Currently Shutdown, Estimated Startup Date:

Notes:

CUMULATIVE UNIT CAPACITY FACTOR (MDC NET) IS CALCULATED WITH A WEIGHTED AVERAGE. MAXIMUM DEPENDABLE CAPACITIES (GROSS \& NET) ARE BASED ON CONDENSER BACKPRESSURE. 


\section{TYPE}

F: Forced

S: scheduled

\section{REASON}

A-Equipment Failure

B-Maintenance or Test

C-Refuel ing

D-Regulatory Restriction

E-Operator Training

License Exemination

F-Administrative

G-Operational Error

$\mathrm{H}$-other

\begin{tabular}{l} 
METHOD \\
\hline 1-Manual \\
2-Manual Scram \\
3-Auto Scram \\
4-Continued \\
5-Reduced Load \\
9-0ther
\end{tabular}

SYSTEM

IEEE Standard

NUREG-0161
COMPONENT

IEEE Standard

$803 A-1983$ and/or

NUREG-0161 Exhibit H 


\section{FACILITY DESCRIPTION}

\section{LOCATION}

STATE.

COUNTY.

MICHIGAN

VANBUREN

\section{DIST AND DIRECTION FROM} NEAREST POPULATION CTR.

TYPE OF REACTOR.

DATE INITIAL CRITICALITY.

MAY 24, 1971

DATE IMITIAL ELECTRICITY...... DECEMBER 31, 1971

DAte COMmercial OPERATE....... December 31, 1971

CONDENSER COOLING METHOO...... COOLING TONERS

CONDENSER COOLING WATER....... LAKE MICHIGAN

ELECTRIC RELIABILITY

COUNCIL................ EAST CENTRAL AREA RELIABILITY

COORDINATION AGREEMENT

\section{UTILITY \& CONTRACTOR INFORMATION}

\section{UTILITY}

LICENSEE.

CORPORATE ADDRESS.

CONTRACTOR

ARCHITECT/ENGINEER

NUC STEAM SYS SUPPLIER

CONSTRUCTOR

TURBINE SUPPLIER

REGULATORY INFORMATION

IE REGION RESPONSIBLE.

IE RESIDENT INSPECTOR

LICENSING PROJ MANAGER

DOCKET MUMBER.

$50-255$

LICENSE \& DATE ISSUANCE....... DPR 020, OCTOBER 16, 1972 


\section{Docket: $50-528$}

OPERATING STATUS

2. Reporting Period: DECEMBER 1994

Outage + On-Line Hrs: $\quad 744.0$

3. Utility Contact: B. S. ECKLUND (602) 393-6221

4. Licensed Thermal Power (MHt):

5. Nameplate Rating (Gross MWe):

6. Design Electrical Rating (Net MHe):

7. Maximum Dependable Capacity (Gross MWe):

8. Maximum Dependable Capacity (Net MUe):

9. If Changes Occurred Above Since Last Report, Give Reasons:

10. Power Level To Which Restricted, If Any (Net MWe):

11. Reasons For Restrictions, If Any:

\begin{tabular}{|c|c|c|c|}
\hline & MONTH & YEAR & CUMULATIVE \\
\hline 12. Report Period Hrs & 744.0 & $8,760.0$ & $78,240.0$ \\
\hline 13. Hours Reactor Critical & 744.0 & $8,675.4$ & $50,632.8$ \\
\hline 14. Rx Reserve Shtdwn Hrs & 0.0 & 0.0 & 0.0 \\
\hline 15. Hrs Generator On-Line & 744.0 & $8,656.7$ & $49,656.6$ \\
\hline 16. Unit Reserve Shtdwn Hrs & 0.0 & 0.0 & 0.0 \\
\hline 17. Gross Therm Ener (MWH) & $2,718,645.0$ & $30,051,470.0$ & $179,082,018.0$ \\
\hline 18. Gross Elec Ener (MUH) & $937,200.0$ & $\overline{10,391,900.0}$ & $62,046,500.0$ \\
\hline 19. Net Elec Ener (MUH) & $886,253.0$ & $9,772,544.0$ & $58,230,904.0$ \\
\hline 20. Unit Service Factor & 100.0 & 98.8 & 63.5 \\
\hline 21. Unit Avail Factor & 100.0 & 98.8 & 63.5 \\
\hline 22. Unit Cap Factor (MDC Net) & 97.6 & 91.4 & 61.0 \\
\hline 23. Unit Cap Factor (DER Net) & 93.8 & 87.8 & 58.6 \\
\hline 24. Unit Forced Outage Rate & 0.0 & 1.2 & 13.5 \\
\hline 25. Forced Outage Hours & 0.0 & 103.3 & $7,782.0$ \\
\hline
\end{tabular}

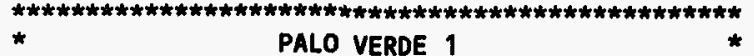

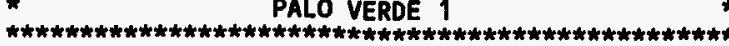

AVERAGE DAILY POWER LEVEL (Net MHe)

\begin{tabular}{lc} 
DAY & PONER \\
\hline 1 & 313 \\
\hline 2 & 1027 \\
\hline 3 & 1223 \\
\hline 4 & 1223 \\
\hline 5 & 1220 \\
\hline 6 & 1218 \\
\hline 7 & 1219 \\
\hline 8 & 1221 \\
\hline 9 & 1222 \\
\hline 10 & 1223 \\
\hline 11 & 1223 \\
\hline 12 & 1222 \\
\hline 13 & 1223 \\
\hline 14 & 1220 \\
\hline 15 & 1221 \\
\hline
\end{tabular}

\begin{tabular}{ll} 
DAY & PONER \\
\hline 16 & 1222 \\
\hline 17 & 1220 \\
\hline 18 & 1222 \\
\hline 19 & 1232 \\
\hline 20 & 1247 \\
\hline 21 & 1248 \\
\hline 22 & 1246 \\
\hline 23 & 1241 \\
\hline 24 & 1243 \\
\hline 25 & 1244 \\
\hline 26 & 1245 \\
\hline 27 & 1244 \\
\hline 28 & 1245 \\
\hline 29 & 1245 \\
\hline 30 & 1246 \\
\hline 31 & 1243 \\
\hline
\end{tabular}

26. Shutdowns Scheduled Over Mext Six Months (Type, Date, Duration): REFUELING OUTAGE, APRIL 1, 1995, 70 DAYS.

27. If Currently Shutdown, Estimated Startup Date:

Notes: 


No. Date Type Hours Reason Method LER Number System Component Cause and Corrective Action To Prevent Recurrence

\begin{tabular}{l} 
TYPE \\
\hline F: Forced \\
S: Scheduled
\end{tabular}

\section{REASON}

A-Equipment Failure

B-Maintenance or Test

C-Refuel ing

D-Regulatory Restriction

E-Operator Training \&

License Examination

F-Administrat ive

G-Operational Error

H-other

\section{METHOO}

1-Manua

2-Manual Scram

3-Auto Scram

4-Cont inued

5-Reduced Load

9-other

\begin{tabular}{|c|c|}
\hline SYSTEM & COMPONENT \\
\hline $\begin{array}{l}\text { IEEE Standard } \\
805-1984 \text { and/or } \\
\text { NUREG-0161 Exhibit F }\end{array}$ & $\begin{array}{l}\text { IEEE Standard } \\
803 \mathrm{~A}-1983 \text { and/or } \\
\text { NUREG-0161 Exhibit H }\end{array}$ \\
\hline
\end{tabular}

NUREG-0161 Exhibit $F$ NUREG-0161 Exhibit $H$ 


\section{FACILITY DESCRIPTION}

LOCATION

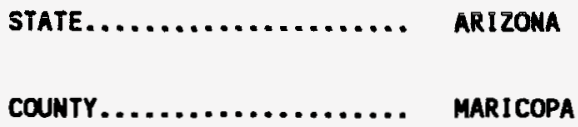

TYPE OF REACTOR............ PUR

DATE INITIAL CRITICALITY...... MAY 25, 1985

DATE INITIAL ELECTRICITY...... JUNE 10, 1985

DATE COMMERCIAL OPERATE....... JAMUARY 28, 1986

CONDEMSER COOLING METHOD...... COOLING TOWER

CONDENSER COOLING MATER....... SEMAGE TREATMENT

ELECTRIC RELIABILITY

COUNCIL................... WESTERN SYSTEMS COORDIMATION

\section{UTILITY \& CONTRACTOR INFORMATION}

\section{UTILITY}

LICENSEE................. ARIzOMA PUBLIC SERVICE CO.

CORPORATE ADDRESS.......... P.O. BOX 52034

PHOENIX, ARIZONA 85072

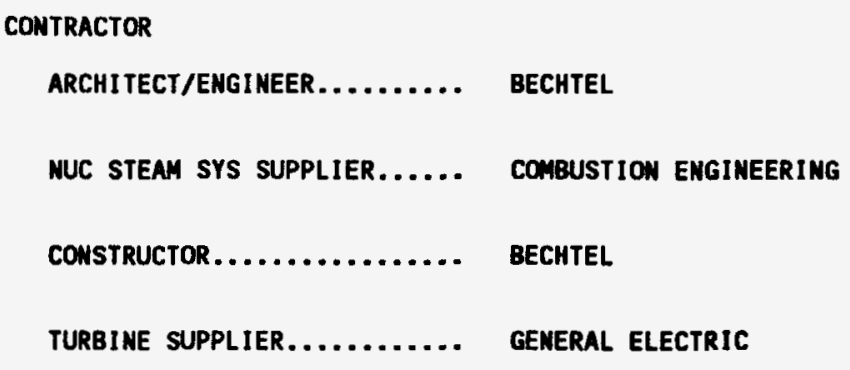

CONTRACTOR

ARCHITECT/ENGINEER......... BECHTEL

MUC STEAM SYS SUPPLIER...... COMBUSTION EMGINEERING

CONSTRUCTOR.

BECHTEL

TURBIHE SUPPLIER.

GENERAL ELECTRIC

\section{REGULATORY INFORMATION}

IE REGION RESPONSIBLE......... 4

IE RESIDENT INSPECTOR

KENMETH JOHNSTON

LICENSING PROJ MANAGER.

TIMOTHY J. POLICH

DOCKET NUMBER

$50-528$

LICENSE \& DATE ISSUANCE. 
1. Docket: $50-529$

OPERATING STATUS

2. Reporting Period: DECEMBER 1994

Outage + On-Line Hrs: $\mathbf{7 4 4 . 0}$

3. Utility Contact: B. S. ECKLUND (602) 393-6221

4. Licensed Thermal Power (MUt):

5. Nameplate Rating (Gross MWe):

6. Design Electrical Rating (Net Mue):

7. Maximum Dependable Capacity (Gross MWe):

8. Maximum Dependable Capacity (Net MHe):

9. If Changes Occurred Above Since Last Report, Give Reasons:

10. Power Level To which Restricted, If Any (Net MHe):

11. Reasons For Restrictions, if Any:

\begin{tabular}{|c|c|c|c|}
\hline & MONTH & YEAR & CUMULATIVE \\
\hline 12. Report Periad Hrs & 744.0 & $8,760.0$ & $72,624.0$ \\
\hline 13. Hours Reactor Critical & 744.0 & $6,103.2$ & $50,651.7$ \\
\hline 14. Rx Reserve Shtdwn Hrs & 0.0 & 0.0 & 0.0 \\
\hline 15. Hrs Generator On-Line & 744.0 & $5,921.0$ & $49,600.9$ \\
\hline 16. Unit Reserve Shtdwn Hrs & 0.0 & 0.0 & 0.0 \\
\hline 17. Gross Therm Ener (MUH) & $2,817,816.0$ & $20,409,662.0$ & $180,243,907.0$ \\
\hline 18. Gross Elec Ener (MUH) & $974,700.0$ & $7,068,500.0$ & $62,731,470.0$ \\
\hline 19. Net Elec Ener (MUH) & $917,052.0$ & $6,573,863.0$ & $58,693,898.0$ \\
\hline 20. Unit Service Factor & 100.0 & 67.6 & 68.3 \\
\hline 21. Unit Avail Factor & 100.0 & 67.6 & 68.3 \\
\hline 22. Unit Cap Factor (MDC Net) & 100.9 & 61.5 & 66.2 \\
\hline 23. Unit Cap Factor (DER Net) & 97.1 & 59.1 & 63.6 \\
\hline 24. Unit Forced Outage Rate & 0.0 & 5.4 & 6.0 \\
\hline 25. Forced Dutage Hours & 0.0 & 339.5 & $3,191.8$ \\
\hline
\end{tabular}

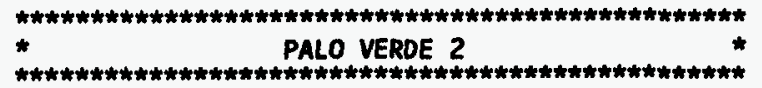

AVERAGE DAILY PONER LEVEL (Net MiNe)

\begin{tabular}{ll} 
DAY & POWER \\
\hline 1 & 1243 \\
\hline 2 & 1241 \\
\hline 3 & 1235 \\
\hline 4 & 1240 \\
\hline 5 & 1236 \\
\hline 6 & 1226 \\
\hline 7 & 1227 \\
\hline 8 & 1228 \\
\hline 9 & 1234 \\
\hline 10 & 1239 \\
\hline 11 & 1240 \\
\hline 12 & 1237 \\
\hline 13 & 1237 \\
\hline 14 & 1237 \\
\hline 15 & 1233 \\
\hline
\end{tabular}

\begin{tabular}{ll}
\hline DAY & POWER \\
\hline 16 & 1232 \\
\hline 17 & 1229 \\
\hline 18 & 1243 \\
\hline 19 & 1242 \\
\hline 20 & 1240 \\
\hline 21 & 1243 \\
\hline 22 & 1241 \\
\hline 23 & 1236 \\
\hline 24 & 1236 \\
\hline 25 & 1238 \\
\hline 26 & 1240 \\
\hline 27 & 1239 \\
\hline 28 & 1240 \\
\hline 29 & 1238 \\
\hline 30 & 1240 \\
\hline 31 & 1238 \\
\hline
\end{tabular}

26. Shutdowns Scheduled Over Next six Months (Type, Date, Duration): REFUELING OUTAGE, FEBRUARY 4, 1995, 60 DAYS.

27. If Currently Shutdown, Est imated Startup Date: Notes: 


\section{FACILITY DESCRIPTION}

\section{LOCATION}

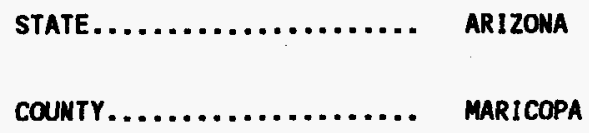

DIST AND DIRECTION FROM

MEAREST POPULATION CTR..... 36 MI W OF PHOENIX, AZ

TYPE OF REACTOR.

DATE INITIAL CRITICALITY

DATE INITIAL ELECTRICITY

DATE COMMERCIAL OPERATE.

CONDENSER COOLING METHOD.

CONDENSER COOLING WATER

ELECTRIC RELIABILITY

COUNCIL.................. MESTERN SYSTEMS COORDINATION

\section{UTILITY \& CONTRACTOR INFORMATION}

\section{UTILITY}

LICENSEE.

CORPORATE ADDRESS.

\section{CONTRACTOR}

ARCHITECT/ENGINEER......... BECHTEL

NUC STEAM SYS SUPPLIER...... COMBUSTION ENGIMEERING

CONSTRUCTOR.............. BECHTEL

TURBINE SUPPLIER.......... GEMERAL ELECTRIC

\section{REGULATORY INFORMATION}

\author{
IE REGION RESPONSIBLE......... \\ IE RESIDENT INSPECTOR.......... KENHETH JOHNSTON \\ LICENSING PROJ MANAGER......... TIMOTHY J. POLICH \\ DOCKET NUMBER.............. 50-529 \\ LICENSE \& DATE ISSUANCE........ NPF 051, APRIL 24, 1986
}


1. Docket: $50-530$

OPERATING STATUS

2. Reporting Period: DECEMBER 1994

3. Utility Contact: 8. S. ECKLUND (602) 393-6221

4. Licensed Thermal Power (MUt):

5. Nameplate Rating (Gross MWe):

6. Design Electrical Rating (Net Mue):

7. Maximum Dependable Capacity (Gross MHe):

8. Maximum Dependable Capacity (Net MHe):

9. If Changes Occurred Above Since Last Report, Give Reasons:

10. Power Level To Which Restricted, If Any (Net MWe):

11. Reasons for Restrictions, If Any:

\begin{tabular}{|c|c|c|c|}
\hline 12. Report Period Hrs & $\begin{array}{l}\text { MONTH } \\
744.0\end{array}$ & $\begin{array}{l}\text { YEAR } \\
8,760.0\end{array}$ & $\begin{array}{l}\text { CUMULAT IVE } \\
61,200.0\end{array}$ \\
\hline 13. Hours Reactor Critical & 348.0 & $5,998.1$ & $45,014.0$ \\
\hline 14. Rx Reserve Shtdun Hrs & 0.0 & 0.0 & 0.0 \\
\hline 15. Hrs Generator On-Line & 337.5 & $5,923.4$ & $44,347.5$ \\
\hline 16. Unit Reserve Shtdwn Hrs & 0.0 & 0.0 & 0.0 \\
\hline 17. Gross Therm Ener (МнH) & $1,224,032.0$ & $21,105,673.0$ & $161,776,913.0$ \\
\hline 18. Gross Elec Ener (MUH) & $424,400.0$ & $7,309,800.0$ & $56,531,700.0$ \\
\hline 19. Net Elec Ener (MUH) & $392,445.0$ & $6,824,487.0$ & $53,122,712.0$ \\
\hline 20. Unit Service Factor & 45.4 & 67.6 & 72.5 \\
\hline 21. Unit Avail Factor & 45.4 & 67.6 & 72.5 \\
\hline 22. Unit Cap Factor (MDC Met) & 43.2 & 63.8 & 71.1 \\
\hline 23. Unit Cap Factor (DER Net) & 41.5 & 61.3 & 68.3 \\
\hline 24. Unit Forced Outage Rate & 0.0 & 1.2 & 6.2 \\
\hline 25. Forced Outage Hours & 0.0 & 74.0 & $2,932.0$ \\
\hline
\end{tabular}

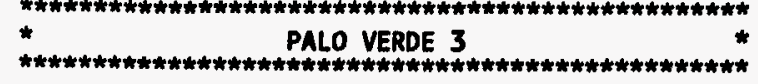

AVERAGe DAILY PONER LeVEL (Net MWe)

\begin{tabular}{lc} 
DAY & PONER \\
\hline 1 & 0 \\
\hline 2 & 0 \\
\hline 3 & 0 \\
\hline 4 & 0 \\
\hline 5 & 0 \\
\hline 6 & 0 \\
\hline 7 & 0 \\
\hline 8 & 0 \\
\hline 9 & 0 \\
\hline 10 & 0 \\
\hline 11 & 0 \\
\hline 12 & 0 \\
\hline 13 & 0 \\
\hline 14 & 0 \\
\hline 15 & 0 \\
\hline
\end{tabular}

\begin{tabular}{cc} 
DAY & POWER \\
\hline 16 & 0 \\
\hline 17 & 8 \\
\hline 18 & 475 \\
\hline 19 & 1190 \\
\hline 20 & 1258 \\
\hline 21 & 1258 \\
\hline 22 & 1259 \\
\hline 23 & 1256 \\
\hline 24 & 1256 \\
\hline 25 & 1255 \\
\hline 26 & 1258 \\
\hline 27 & 1259 \\
\hline 28 & 1259 \\
\hline 29 & 1259 \\
\hline 30 & 1261 \\
\hline 31 & 1260 \\
\hline
\end{tabular}

26. Shutdowns Scheduled Over Next six Months (Type, Date, Duration):

27. If Currently Shutdown, Estimated Startup Date:

Notes: 


\begin{tabular}{|c|c|c|c|c|c|c|c|c|c|}
\hline No. & Date & Type & Hours & Reason & Method & LER Number & System & Component & Cause and Corrective Action To Prevent Recurrence \\
\hline $94-04$ & $11 / 26 / 94$ & $\mathbf{s}$ & 406.5 & B & 4 & & & & $\begin{array}{l}\text { CONTINUATION OF PLANMED MID-CYCLE OUTAGE FOR } \\
\text { STEAM GENERATOR TUBE EDDY CURREMT INSPECTION. }\end{array}$ \\
\hline
\end{tabular}

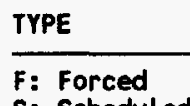

F: Forced

S: Scheduled

\begin{tabular}{l} 
REASON \\
\hline A-Equipment Failure \\
B-Maintenance or Test \\
C-Refuel ing \\
D-Regulatory Restriction \\
E-Operator Training \& \\
License Examination \\
F-Administrat ive \\
G-Operational Error \\
H-Other
\end{tabular}

METHOO

1-Manual

2-Manual Scram

3-Auto Scram

4-Cont inued

5-Reduced Load

9-0ther

G-Operational Error 


\section{FACILITY DESCRIPTION}

\section{LOCATION}

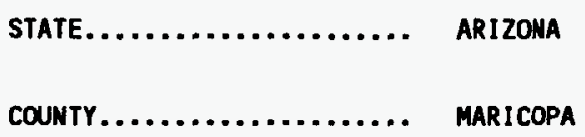

\section{DIST AND DIRECTION FROM} NEAREST POPULATION CTR.....

36 MI W OF PHOENIX, AZ

TYPE OF REACTOR

DATE INITIAL CRITICALITY......

DATE INITIAL ELECTRICITY......

DATE COMMERCIAL OPERATE.......

CONDENSER COOLING METHOD.......

CONDENSER COOLING WATER....... SEWAGE TREATMENT

ELECTRIC RELIABILITY

COUNCIL................... WESTERN SYSTEMS COORDINATION COUNCIL

\section{UTILITY \& CONTRACTOR INFORMATION}

UTILITY

LICENSEE............... ARIZONA PUBLIC SERVICE CO.

CORPORATE ADDRESS

P.0. BOX 52034

PHOENIX, ARIZONA 85072

\section{CONTRACTOR}

ARCHITECT/EMGIMEER......... BECHTEL

MUC STEAM SYS SUPPLIER..... COMBUSTION ENGINEERING

CONSTRUCTOR............... BECHTEL

TURBINE SUPPLIER........... GEMERAL ELECTRIC

\section{REGULATORY INFORMATION}

IE REGION RESPONSIBLE........ 4

IE RESIDENT INSPECTOR.......... KENMETH JOHNSTON

LICENSING PROJ MANAGER......... CHARLES M. TRAMMELL III

DOCKET MUMBER............ 50-530

LICENSE \& DATE ISSUANCE........ NPF 074, MOVEMBER 25, 1987 
1. Docket: $50-277$

OPERATING STATUS

2. Reporting Period: DECEMBER 1994 Outage + On-Line Hrs: $\mathbf{7 4 4 . 0}$

3. Utility Contact: W. J. JEFFREY (717) 456-7014 EXT. 4027

4. Licensed Thermal Power (MWt):

5. Nameplate Rating (Gross MWe):

6. Design Electrical Rating (Net MHe):

7. Maximum Dependable Capacity (Gross MMe):

8. Maximum Dependable Capacity (Net MHe):

9. If Changes Occurred Above since Last Report, Give Reasons:

As a ResUlt of RERATING, ITEMS 5, 7, AND 8 haVe INCREASED. THE NEW VALUE FOR ITEM 6 HAS NOI YET BEEN DÉTERMINED.

10. Power Level To thich Restricted, If Any (Het MHe):

11. Reasons for Restrictions, If Any:

\begin{tabular}{|c|c|c|c|}
\hline & MONTH & YEAR & CUMULATIVE \\
\hline 12. Report Period Hrs & 744.0 & $8,760.0$ & 179.640 .0 \\
\hline 13. Hours Reactor Critical & 744.0 & 7.851 .0 & $113,963.1$ \\
\hline 14. Rx Reserve Shtchn Hrs & 0.0 & 0.0 & 0.0 \\
\hline 15. Hrs Generator On-Line & 744.0 & $7,783.0$ & $110,003.2$ \\
\hline 16. Unit Reserve Shtdun Hrs & 0.0 & 0.0 & 0.0 \\
\hline 17. Gross Therm Ener (MUH) & $2,572,063.0$ & $23,831,390.0$ & $328,320,883.0$ \\
\hline 18. Gross Elec Ener (MUH) & $861,000.0$ & $7,727,900.0$ & $107,879,390.0$ \\
\hline 19. Net Elec Ener (MWH) & $840,017.0$ & $7,451,654.0$ & $103,442,456.0$ \\
\hline 20. Unit Service Factor & 100.0 & 88.8 & 61.2 \\
\hline 21. Unit Avail Factor & 100.0 & 88.8 & 61.2 \\
\hline 22. Unit Cap Factor (MDC Net) & 103.3 & 77.8 & 52.7 \\
\hline 23. Unit Cap Factor (DER Net) & 106.0 & 79.9 & 54.1 \\
\hline 24. Unit Forced Outage Rate & 0.0 & 1.5 & 13.1 \\
\hline 25. Forced Outage Hours & 0.0 & 121.0 & $16,587.0$ \\
\hline
\end{tabular}

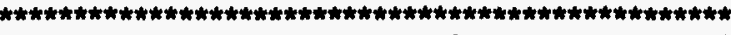 \\ * PEACH BOTTOM 2 .}

AVERAGE DAILY POWER LeVEL (Net Mue)

\begin{tabular}{ll} 
DAY & POWER \\
\hline 1 & 1126 \\
\hline 2 & 1125 \\
\hline 3 & 1130 \\
\hline 4 & 1130 \\
\hline 5 & 1126 \\
\hline 6 & 1134 \\
\hline 7 & 1130 \\
\hline 8 & 1122 \\
\hline 9 & 1134 \\
\hline 10 & 1130 \\
\hline 11 & 1130 \\
\hline 12 & 1130 \\
\hline 13 & 1130 \\
\hline 14 & 1134 \\
\hline 15 & 1126 \\
\hline
\end{tabular}

\begin{tabular}{ll} 
DAY & POWER \\
\hline 16 & 1130 \\
\hline 17 & 1126 \\
\hline 18 & 1130 \\
\hline 19 & 1126 \\
\hline 20 & 1130 \\
\hline 21 & 1130 \\
\hline 22 & 1130 \\
\hline 23 & 1126 \\
\hline 24 & 1134 \\
\hline 25 & 1126 \\
\hline 26 & 1134 \\
\hline 27 & 1126 \\
\hline 28 & 1130 \\
\hline 29 & 1130 \\
\hline 30 & 1122 \\
\hline 31 & 1130 \\
\hline
\end{tabular}

26. Shutdowns Scheduled Over Next Six Months (Type, Date, Duration):

27. If Currently Shutdown, Estimated Startup Date:

Notes:

LICENSEE REVISED JANUARY 1994 NET ELECTRICAL ENERGY FROM $762,627.0$ TO $763,627.0$. 


\section{FACILITY DESCRIPTION}

LOCATION

STATE

COUNTY.

\section{DIST AND DIRECTION FROM}

MEAREST POPUL

TYPE OF REACTOR.

DATE INITIAL CRITICALITY...... SEPTEMBER 16, 1973

DATE INITIAL ELECTRICITY...... FEBRUARY 18, 1974

DATE COMNERCIAL OPERATE....... JULY 05, 1974

CONDENSER COOLING METHOO....... ONCE THRU

CONDENSER COOLING WATER....... SUSQUEHANHA RIVER

ELECTRIC RELIABILITY

COUNCIL.................... MID-ATLANTIC AREA COUNCIL

\section{UTILITY \& CONTRACTOR INFORMATION}

UTILITY

LICENSEE. .

PECO ENERGY COMPANY

CORPORATE ADDRESS.

2301 MARKET STREET

PHILAdELPHIA, PENwSYlvania 19154

\section{CONTRACTOR}

ARCHITECT/ENGINEER......... BECHTEL

NUC STEAM SYS SUPPLIER...... GEMERAL ELECTRIC

CONSTRUCTOR............... BECHTEL

TURBINE SUPPLIER........... GENERAL ELECTRIC

REGULATORY INFORMATION

IE REGION RESPONSIBLE......... 1

IE RESIDENT INSPECTOR......... WAYME SCHMIDT

LICENSING PROJ MANAGER......... JOSEPH W. SHEA

DOCKET MUMBER .

$50-277$

LICENSE \& DATE ISSUANCE

DPR 044, DECEMBER 14, 1973 
1. Docket: $50-278$

OPERATING STATUS

2. Reporting Period: DECEMBER 1994

Outage + On-Line Hrs: $\mathbf{7 4 4 . 0}$

3. Utility Contact: W. J. JEFFREY (717) 456-7014 EXT. 4027

4. Licensed Thermal Power (MHt):

5. Nameplate Rating (Gross MWe):

6. Design Electrical Rating (Net MWe):

7. Maximum Dependable Capacity (Gross MWe):

8. Maximum Dependable Capacity (Net MHe):

9. If Changes Occurred Above Since Last Report, Give Reasons:

10. Power Level To Which Restricted, If Any (Net MWe):

11. Reasons For Restrictions, If Any:

\begin{tabular}{|c|c|c|c|}
\hline & MONTH & YEAR & CUMULAT IVE \\
\hline 12. Report Period Hrs & 744.0 & $8,760.0$ & $175,536.0$ \\
\hline 13. Hours Reactor Critical & 744.0 & B,588.0 & $113,259.4$ \\
\hline 14. Rx Reserve Shtdwn Hrs & 0.0 & 0.0 & 0.0 \\
\hline 15. Hrs Generator on-Line & 744.0 & $8,588.0$ & $109,879.2$ \\
\hline 16. Unit Reserve Shtdwn Hrs & 0.0 & 0.0 & 0.0 \\
\hline 17. Gross Therm Ener (MWH) & $2,438,916.0$ & $27,800,466.0$ & $326,573,943.0$ \\
\hline 18. Gross Elec Ener (MWH) & $810,300.0$ & $9,155,900.0$ & $107,146,032.0$ \\
\hline 19. Net Elec Ener (MWH) & $789,630.0$ & $8,867,352.0$ & $102,841,588.0$ \\
\hline 20. Unit Service Factor & 100.0 & 98.0 & 62.6 \\
\hline 21. Unit Avail Factor & 100.0 & 98.0 & 62.6 \\
\hline 22. Unit Cap Factor (MDC Net) & 102.5 & 97.8 & 56.6 \\
\hline 23. Unit Cap Factor (DER Net) & 99.7 & 95.0 & 55.0 \\
\hline 24. Unit Forced Outage Rate & 0.0 & 2.0 & 11.6 \\
\hline 25. Forced Outage Hours & 0.0 & 172.0 & $14,357.7$ \\
\hline
\end{tabular}

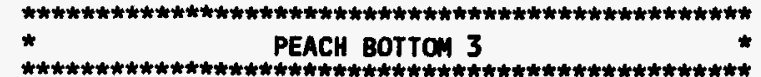

AVERAGE DAILY PONER LEVEL (Net me)

\begin{tabular}{ll} 
DAY & PONER \\
\hline 1 & 1063 \\
\hline 2 & 1063 \\
\hline 3 & 1068 \\
\hline 4 & 1068 \\
\hline 5 & 1068 \\
\hline 6 & 1068 \\
\hline 7 & 1072 \\
\hline 8 & 1060 \\
\hline 9 & 1072 \\
\hline 10 & 1068 \\
\hline 11 & 1063 \\
\hline 12 & 1067 \\
\hline 13 & 1066 \\
\hline 14 & 1062 \\
\hline 15 & 1067 \\
\hline
\end{tabular}

\begin{tabular}{cc} 
DAY & PONER \\
\hline 16 & 1063 \\
\hline 17 & 933 \\
\hline 18 & 1069 \\
\hline 19 & 1064 \\
\hline 20 & 1064 \\
\hline 21 & 1069 \\
\hline 22 & 1068 \\
\hline 23 & 1064 \\
\hline 24 & 1064 \\
\hline 25 & 1068 \\
\hline 26 & 1060 \\
\hline 27 & 1060 \\
\hline 28 & 1069 \\
\hline 29 & 1064 \\
\hline 30 & 1064 \\
\hline 31 & 1060 \\
\hline
\end{tabular}

26. Shutdowns Scheduled Over Next Six Months (Type, Date, Duration):

27. If Currently Shutdown, Estimated Startup Date:

Notes: 


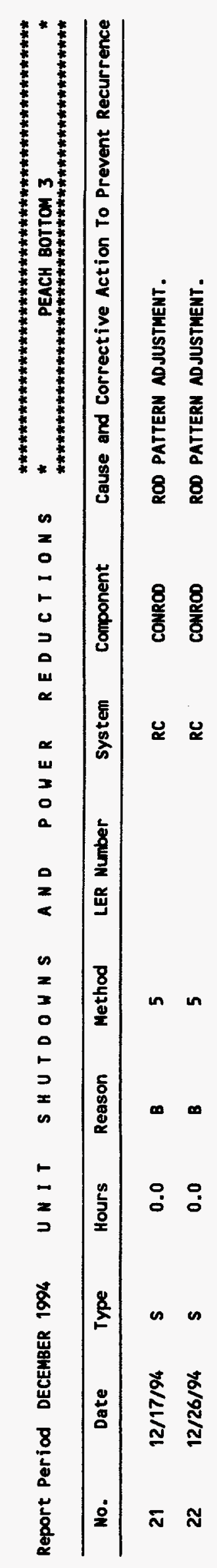

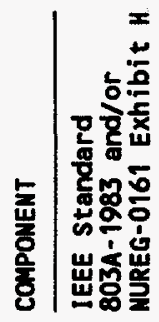

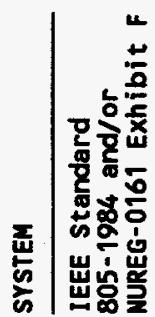

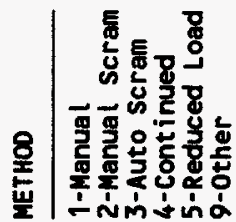

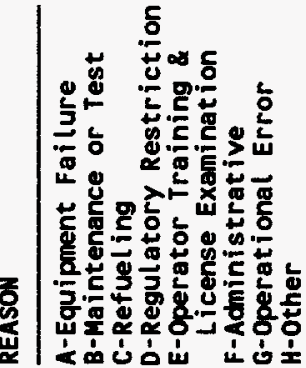

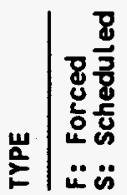




\section{FACILITY DESCRIPTION}

\section{LOCATION}

STATE..................

COUNTY.

DIST AND DIRECTION FROM

NEAREST POPULATION CTR..... 17.9 MI S. OF LANCASTER, PA

TYPE OF REACTOR

BUR

DATE INITIAL CRITICALITY...... AUGUST 07, 1974

DATE INITIAL ELECTRICITY...... SEPTEMBER 01, 1974

DATE COMMERCIAL OPERATE....... DeCEMBER 23, 1974

CONDENSER COOLING METHOO...... ONCE THRU

CONDENSER COOLING WATER.

SUSQUEHAMHA RIVER

ELECTRIC RELIABILITY

COUNCIL................

mid-ATLANTIC AREA COUNCIL

\section{UTILITY \& CONTRACTOR INFORMATION}

UTILITY

LiCENSEE.................. PECO EMERgY COMPAMY

CORPORATE ADDRESS

2301 MARKET STREET

PHILAdelPhIA, PENHSYlVania 19154

\section{CONTRACTOR}

ARCHITECT/ENGINEER......... BECHTEL

NUC STEAM SYS SUPPLIER...... GEMERAL ELECTRIC

CONSTRUCTOR............... BECHTEL

TURBIME SUPPLIER.......... GENERAL ELECTRIC

\section{REGULATORY IMFORMATION}

IE REGION RESPONSIBLE......... 1

IE RESIDENT INSPECTOR......... WAYME SCHMIDT

LICENSING PROJ MANAGER......... JOSEPH W. SHEA

DOCKET MUMBER.............. 50-278

LICENSE \& DATE ISSUANCE........ DPR 056, JULY 02, 1974 
1. Docket: $50-440$

OPERATING STATUS

2. Reporting Period: DECEMBER 1994

Outage + On-Line Hrs:

744.0

3. Utility Contact: G. M. MEUMAN (216) 280-5815

4. Licensed Thermal Power (MWt):

5. Nameplate Rating (Gross Mue):

6. Design Electrical Rating (Net MHe):

7. Maximum Dependable Capacity (Gross MUe):

8. Maximum Dependable Capacity (Net MHe):

\begin{tabular}{l}
\hline 3579 \\
\hline 1250 \\
\hline 1191 \\
\hline 1225 \\
\hline 1166
\end{tabular}

9. If Changes Occurred Above Since Last Report, Give Reasons:

10. Power Level To Which Restricted, If Any (Net MWe):

11. Reasons For Restrictions, If Any:

\begin{tabular}{|c|c|c|c|}
\hline & MONTH & YEAR & CUMULATIVE \\
\hline 12. Report Period Hrs & 744.0 & $8,760.0$ & $62,412.0$ \\
\hline 13. Hours Reactor Critical & 744.0 & $4,398.9$ & $41,930.3$ \\
\hline 14. Rx Reserve Shtdwn Hrs & 0.0 & 0.0 & 0.0 \\
\hline 15. Hrs Generator On-Line & 744.0 & $4,152.4$ & $40,288.0$ \\
\hline 16. Unit Reserve Shtdwn Hrs & 0.0 & 0.0 & 0.0 \\
\hline 17. Gross Therm Ener (MUH) & $2,285,870.0$ & $14,115,147.0$ & $136,995,907.0$ \\
\hline 18. Gross Elec Ener (MUH) & $777,982.0$ & $4,824,754.0$ & $47,049,746.0$ \\
\hline 19. Net Elec Ener (MWH) & $742,331.0$ & $4,591,898,0$ & $44,684,827.0$ \\
\hline 20. Unit Service Factor & 100.0 & 47.4 & 64.6 \\
\hline 21. Unit Avail Factor & 100.0 & 47.4 & 64.6 \\
\hline 22. Unit Cap Factor (MDC Met) & 85.6 & 45.0 & 62.0 \\
\hline 23. Unit Cap Factor (DER Net) & 83.8 & 44.0 & 60.1 \\
\hline 24. Unit Forced Outage Rate & 0.0 & 1.3 & 11.4 \\
\hline 25. Forced Outage Hours & 0.0 & 56.2 & $5,195.6$ \\
\hline
\end{tabular}

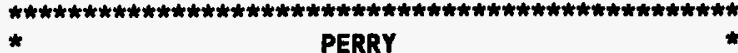

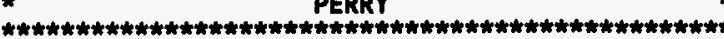

AVERAGE DAILY POWER LEVEL (Net MWe)

\begin{tabular}{ll} 
DAY & PONER \\
\hline 1 & 1192 \\
\hline 2 & 1187 \\
\hline 3 & 1185 \\
\hline 4 & 1160 \\
\hline 5 & 1183 \\
\hline 6 & 1188 \\
\hline 7 & 1191 \\
\hline 8 & 1194 \\
\hline 9 & 1192 \\
\hline 10 & 900 \\
\hline 11 & 899 \\
\hline 12 & 920 \\
\hline 13 & 902 \\
\hline 14 & 885 \\
\hline 15 & 884 \\
\hline
\end{tabular}

\begin{tabular}{cc} 
DAY & PONER \\
\hline 16 & 883 \\
\hline 17 & 895 \\
\hline 18 & 941 \\
\hline 19 & 919 \\
\hline 20 & 903 \\
\hline 21 & 887 \\
\hline 22 & 886 \\
\hline 23 & 959 \\
\hline 24 & 966 \\
\hline 25 & 966 \\
\hline 26 & 971 \\
\hline 27 & 910 \\
\hline 28 & 906 \\
\hline 29 & 904 \\
\hline 30 & 989 \\
\hline 31 & 986 \\
\hline
\end{tabular}

26. Shutdowns Scheduled Over Mext Six Months (Type, Date, Duration):

27. If Currently Shutdown, Estimated Startup Date:

Notes:

CUMULATIVE UMIT CAPACITY FACTOR (MDC MET) IS CALCULATED MITH A MEIGHTED AVERAGE. 


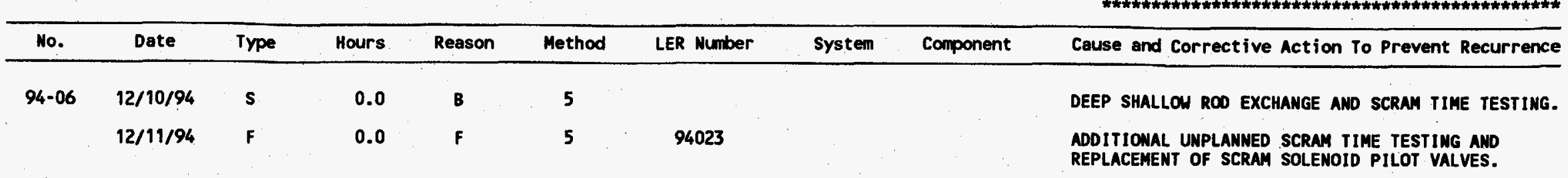

\begin{tabular}{|c|c|}
\hline TYPE & REASON \\
\hline $\begin{array}{l}\text { F: Forced } \\
\text { S: Scheduled }\end{array}$ & $\begin{array}{l}\text { A-Equipment Failure } \\
\text { B-Maintenance or Test } \\
\text { C-Refuel ing } \\
\text { D-Regulatory Restriction } \\
\text { E-Operator Iraining \& } \\
\text { License Examination } \\
\text { F-Administrat ive } \\
\text { G-Operat ional Error } \\
\text { H-Other }\end{array}$ \\
\hline
\end{tabular}

\section{METHOD}

1-Manual

2-Manual Scran

3-Auto Scra

4-Cont inved

9-Reduced Load

-Other

\section{SYSTEM}

IEEE Standard
$805-1984$ and/or

MUREG-0161 Exhibit $F$

\section{COMPONENT}

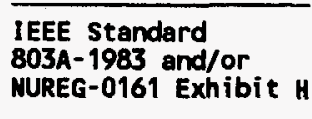

NUREG-0161 Exhibit H

Gdininistrat ive

H-other 


\section{FACILITY DESCRIPTION}

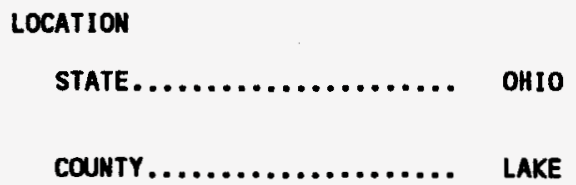

\section{UTILITY \& CONTRACTOR INFORMATION}

UTILITY

LICENSEE................ Cleveland EleCtric Illuminating co.

CORPORATE ADDRESS.......... P.O. BOX 5000

$$
\begin{aligned}
& \text { P.O. BOX } 5000 \\
& \text { CLEVELAND, OHIO } 44136
\end{aligned}
$$

\section{CONTRACTOR}

ARCHITECT/ENGINEER .

\section{GILBERT ASSOCIATES}

NUC STEAM SYS SUPPLIER.

GENERAL ELECTRIC

CONSTRUCTOR

KAISER ENGINEERS

TURBINE SUPPLIER

GENERAL ELECTRIC

\section{REGULATORY INFORMATIO}

IE REGION RESPONSIBLE........ 3

IE RESIDENT INSPECTOR.

DONALD KOSLOFF

LICENSING PROJ MANAGER.

JON B. HOPKINS

DOCKET NUMBER

$50-440$

LICENSE \& DATE ISSUANCE. 
1. Docket: $50-293$

OPERATING STATUS

2. Reporting Period: DECEMBER 1994

Outage + On-Line Hrs:

744.0

3. Utility Contact: H. MUNRO (508) $830-8474$

4. Licensed Thermal Power (MUt):

5. Nameplate Rating (Gross MHe):

6. Design Electrical Rating (Net MWe):

7. Maximum Dependable Capacity (Gross MWe):

8. Maximum Dependable Capacity (Net MHe):

\begin{tabular}{c}
\hline 1998 \\
\hline 678 \\
\hline 655 \\
\hline 696 \\
\hline 670
\end{tabular}

9. If Changes Occurred Above Since Last Report, Give Reasons:

10. Power Level To thich Restricted, If Any (Net MWe):

11. Reasons For Restrictions, If Any:

\begin{tabular}{|c|c|c|c|}
\hline & MONTH & YEAR & CUMULATIVE \\
\hline 12. Report Period Hrs & 744.0 & $8,760.0$ & $193,392.0$ \\
\hline 3. Hours Reactor Critical & 744.0 & $6,258.6$ & $119,200.1$ \\
\hline 4. Rx Reserve Shtdun Hrs & 0.0 & 0.0 & 0.0 \\
\hline 5. Hrs Generator On-Line & 709.1 & $6,072.1$ & $114,861.1$ \\
\hline 16. Unit Reserve Shtdwn Hrs & 0.0 & 0.0 & 0.0 \\
\hline 17. Gross Therm Ener (ММн) & $1,332,240.0$ & $11,610,480.0$ & $202,755,216.0$ \\
\hline 18. Gross Elec Ener (MUH) & $454,430.0$ & $3,973,740.0$ & $68,619,674.0$ \\
\hline 19. Net Elec Ener (MUK) & $437,127.0$ & $3,824,083.0$ & $65,957,777.0$ \\
\hline 20. Unit Service Factor & 95.3 & 69.3 & 59.4 \\
\hline 21. Unit Avail Factor & 95.3 & 69.3 & 59.4 \\
\hline 22. Unit Cap Factor (MDC Net) & 87.7 & 65.2 & 50.9 \\
\hline 23. Unit Cap Factor (DER Net) & 89.7 & 66.6 & 52.1 \\
\hline 4. Unit forced Outage Rate & 4.7 & 22.3 & 12.4 \\
\hline 5. Forced Outage Hours & 34.9 & 1745.9 & $16,313.1$ \\
\hline
\end{tabular}

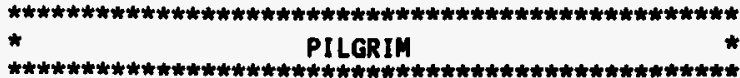

AVERAGE DAILY PONER LEVEL (Net MWe)

\begin{tabular}{lc} 
DAY & POWER \\
\hline 1 & 62 \\
\hline 2 & 85 \\
\hline 3 & 68 \\
\hline 4 & 485 \\
\hline 5 & 656 \\
\hline 6 & 594 \\
\hline 7 & 645 \\
\hline 8 & 664 \\
\hline 9 & 659 \\
\hline 10 & 664 \\
\hline 11 & 663 \\
\hline 12 & 665 \\
\hline 13 & 666 \\
\hline 14 & 666 \\
\hline 15 & 665 \\
\hline
\end{tabular}

\begin{tabular}{cc} 
DAY & PONER \\
\hline 16 & 665 \\
\hline 17 & 609 \\
\hline 18 & 414 \\
\hline 19 & 641 \\
\hline 20 & 664 \\
\hline 21 & 666 \\
\hline 22 & 665 \\
\hline 23 & 666 \\
\hline 24 & 665 \\
\hline 25 & 665 \\
\hline 26 & 664 \\
\hline 27 & 665 \\
\hline 28 & 665 \\
\hline 29 & 664 \\
\hline 30 & 665 \\
\hline 31 & 665 \\
\hline
\end{tabular}

26. Shutdowns Scheduled Over Next six Months (Type, Date, Duration): REFUELING OUTAGE, MARCH 25, 1995, 55 DAYS.

27. If Currently Shutdown, Estimated Startup Date: Notes: 
Report Period DECEMBER 1994

UNIT SHUTDOHNS AND POWER REDUCTIONS

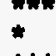

PILGRIM

\begin{tabular}{|c|c|c|c|c|c|c|c|c|}
\hline No. & Date & Type & Hours & Reason & Method & LER Number & System & Cause and Corrective Action To Prevent Recurrence \\
\hline
\end{tabular}

$\begin{array}{llllll}10 & 12 / 03 / 94 & F & 34.9 & B & 1\end{array}$

$\begin{array}{llllll}11 & 12 / 18 / 94 & S & 0.0 & B & 5\end{array}$

MAIN GEMERATOR MANUALLY TRIPPED TO PERFORM BALANCING.

PONER REDUCTION TO PERFORM THERMAL BACKMASH OF THE MAIN CONDENSER.

\begin{tabular}{|c|c|}
\hline TYPE & REASON \\
\hline $\begin{array}{l}\text { F: Forced } \\
\text { s: Scheduled }\end{array}$ & $\begin{array}{l}\text { A-Equipment Failure } \\
\text { B-Maintenance or Test } \\
\text { C-Refuel ing } \\
\text { D-Regulatory Restriction } \\
\text { E-operator Training \& } \\
\text { License Examination } \\
\text { F-Administrat ive } \\
\text { G-Operational Error } \\
\text { H-Other }\end{array}$ \\
\hline
\end{tabular}

\begin{tabular}{l} 
METHOD \\
\hline 1-Manual \\
2-Manual Scram \\
3-Auto Scram \\
4-Cont inued \\
5-Reduced Load \\
9-Other
\end{tabular}

SYSTEM
IEEE Standard
$805-1984$ and/or

805-1984 and/or

NUREG-0161 Exhibit $F$
COMPONENT

IEEE Standard

803A-1983 and/or
NUREG-0161 Exhibit H

G-Operational Error

$\mathrm{H}$-Other 


\section{FACILITY DESCRIPTION}

\section{LOCATION}

STATE................. MASSACHUSETTS

COUNTY.................. PLYMOUTH

\section{DIST AND DIRECTION FRO}

MEAREST POPULATION CTR..... $4 \mathrm{MI}$ SE OF PLYMOUTH, MA

TYPE OF REACTOR............ BWR

DATE INITIAL CRITICALITY...... JUNE 16, 1972

DATE INITIAL ELECTRICITY...... JULY 19, 1972

DATE COMmercial OPERATE....... December 01, 1972

CONDENSER COOLING METHOO...... ONCE THRU

CONDENSER COOLING WATER....... CAPE COD BAY

ELECTRIC RELIABILITY

COUNCIL.................. $\begin{aligned} & \text { NORTHEASTERN POWER } \\ & \text { COORDINATION COUNCII }\end{aligned}$

\section{UTILITY \& CONTRACTOR INFORMATION}

\section{UTILITY}

LICENSEE................ BOSTON EDISON CO.

CORPORATE ADDRESS.

800 BOYLSTON STREET

BOSTON, MASSACHUSETTS 02199

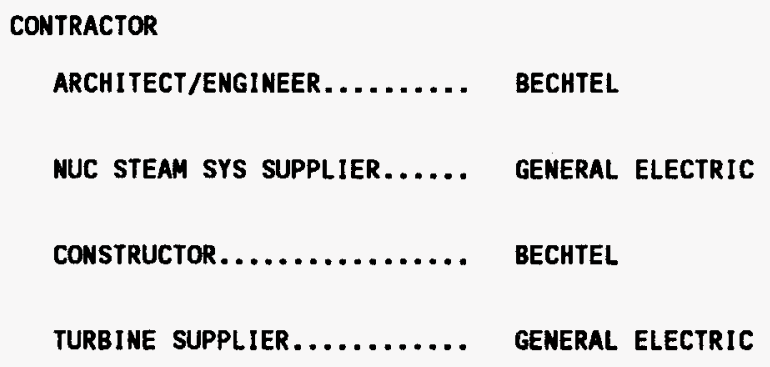

TURBINE SUPPLIER

GENERAL ELECTRIC

\section{REGULATORY INFORMATION}

IE REGION RESPONSIBLE........ 1
IE RESIDENT INSPECTOR........ JOHN B. MACDONALD
LICENSING PROJ MANAGER....... RONALD B. EATON
DOCKET MUMBER............... $50-293$
LICENSE \& DATE ISSUANCE....... DPR 035, SEPTEMBER 15, 1972 
1. Docket: $50-266$

OPERATING STATUS

2. Reporting Period: DECEMBER 1994

Outage + On-Line Hrs: $\mathbf{7 4 4 . 0}$

3. Utility Contact: M. B. KOUDELKA (414) $755-6480$

4. Licensed Thermal Power (MWt):

5. Nameplate Rating (Gross MWe):

6. Design Electrical Rating (Net MHe):

7. Maximum Dependable Capacity (Gross MWe):

8. Maximum Dependable Capacity (Net MWe):

9. If Changes Occurred Above Since Last Report, Give Reasons:

10. Power Level To Which Restricted, If Any (Net MUe):

11. Reasons For Restrictions, If Any:

\begin{tabular}{|c|c|c|c|}
\hline & MONTH & YEAR & CUMULATIVE \\
\hline 12. Report Period Hrs & 744.0 & $8,760.0$ & $211,728.0$ \\
\hline 13. Hours Reactor Critical & 730.1 & $8,120.7$ & $176,839.6$ \\
\hline 14. Rx Reserve Shtdwn Hrs & 0.0 & 0.0 & 667.3 \\
\hline 15. Hrs Generator On-Line & 730.1 & $8,072.2$ & $173,712.4$ \\
\hline 16. Unit Reserve Shtdwn Hrs & 0.0 & 0.0 & 846.9 \\
\hline 17. Gross Therm Ener (MWH) & $1,096,432.0$ & $12,063,227.0$ & $245,544,997.0$ \\
\hline 18. Gross Elec Ener (MWH) & 371.760 .0 & $4,084,520.0$ & $82,938,650.0$ \\
\hline 19. Net Elec Ener (MUH) & $356,154.0$ & $3,905,061.0$ & $79,060,651.0$ \\
\hline 20. Unit Service Factor & 98.1 & 92.1 & 82.0 \\
\hline 21. Unit Avail Factor & 98.1 & 92.1 & 82.4 \\
\hline 22. Unit Cap Factor (MDC Net) & 98.7 & 91.9 & 76.6 \\
\hline 23. Unit Cap Factor (DER Net) & 96.3 & 89.7 & 75.1 \\
\hline 24. Unit Forced Outage Rate & 0.0 & 0.0 & 1.5 \\
\hline 25. Forced Outage Hours & 0.0 & 0.0 & $2,653.3$ \\
\hline
\end{tabular}

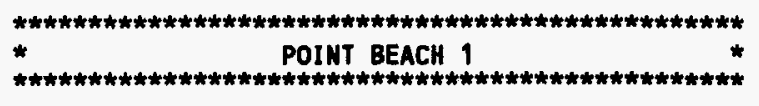

AVERAGE DAILY POWER LEVEL (Net MHe)

\begin{tabular}{|c|c|c|c|}
\hline DAY & POMER & DAY & POWER \\
\hline 1 & 494 & 16 & 495 \\
\hline 2 & 494 & 17 & 494 \\
\hline 3 & 494 & 18 & 497 \\
\hline 4 & 483 & 19 & 495 \\
\hline 5 & 494 & 20 & 496 \\
\hline 6 & 493 & 21 & 496 \\
\hline 7 & 494 & 22 & 496 \\
\hline 8 & 494 & 23 & 496 \\
\hline 9 & 493 & 24 & 495 \\
\hline 10 & 493 & 25 & 465 \\
\hline 11 & 494 & 26 & 494 \\
\hline 12 & 493 & 27 & 495 \\
\hline 13 & 493 & 28 & 495 \\
\hline 14 & 494 & 29 & 494 \\
\hline 15 & 496 & 30 & 488 \\
\hline & & 31 & 52 \\
\hline
\end{tabular}

26. Shutdouns Scheduled Over Next Six Months (Type, Date, Duration): REFUELING OUTAGE, MARCH 11, 1995, 36 DAYS.

27. If Currently Shutdown, Est imated Startup Date:

Notes: DATA REPORTED AND FACTORS CALCULATED AS REQUESTED IN MRC LETTER
DATED SEPTEMBER 22, 1977 . CUMULATIVE UNIT CAPACITY FACTOR (MDC NET) IS CALCULATED WITH A MEIGHTED AVERAGE. 


\begin{tabular}{|c|c|}
\hline TYPE & REASON \\
\hline $\begin{array}{l}\text { F: Forced } \\
\text { S: Scheduled }\end{array}$ & $\begin{array}{l}\text { A-Equipment Failure } \\
\text { B-Maintenance or Test } \\
\text { C-Refuel ing } \\
\text { D-Regulatory Restriction } \\
\text { E-Operator Training \& } \\
\text { License Examination } \\
\text { F-Administrat ive } \\
\text { G-Operational Error } \\
\text { H-Other }\end{array}$ \\
\hline
\end{tabular}

\section{METHOD}

1-Manual

2-Manual Scran

3-Auto Scram

4-Cont inued

-Reduced Load

9-other

\section{SYSTEM \\ IEEE Standard \\ 805-1984 and/or
MUREG-0161 Exhibit $F$}

-Administrative

H-Other 


\section{FACILITY DESCRIPTION}

\section{LOCATION}

STATE................. MISCOHSIN
COUNTY............... MANITONOC

DIST AND DIRECTION FROM

NEAREST POPULATION CTR..... 13 MI NNH OF MANITONOC, WI

TYPE OF REACTOR

PWR

DATE INITIAL CRITICALITY...... NOVEMBER 02, 1970

DATE INITIAL ELECTRICITY...... NOVEMBER 06, 1970

DATE COMmercial OPERATE....... DeCEMBER 21, 1970

CONDENSER COOLING METHOD...... ONCE THRU

CONDENSER COOLING MATER....... LAKE MICHIGAN

ELECTRIC RELIABILITY

COUNCIL.................. MID-AMERICA INTERPOOL NETHORK

\section{UTILITY \& CONTRACTOR INFORMATION}

UTILITY

LICENSEE................. HISCONSIN ELECTRIC POWER CO.

CORPORATE ADDRESS.......... 231 WEST MICHIGAN STREET

MILHAUKEE, HISCONSIN 53201

\section{CONTRACTOR}

ARCHI TECT/ENGIMEER......... BECHTEL

MUC STEAM SYS SUPPLIER...... VESTINGHOUSE

CONSTRUCTOR............... BECHTEL

TURBINE SUPPLIER.......... WESTIMGHOUSE

\section{REGULATORY IHFORMATIOW}

IE REGION RESPONSIBLE.......... 3

IE RESIDENT INSPECTOR.......... TIMOTHY KOBETZ

LICENSING PROJ MANAGER......... ALLEN G. HANSEN

DOCKET NUMBER............. 50-266

LICENSE \& DATE ISSUANCE........ DPR 024, OCTOBER 05, 1970 
1. Docket: $50-301$

OPERATING STATUS

2. Reporting Period: DECEMBER 1994

Outage + On-Line Hrs: $\quad \mathbf{7 4 4 . 0}$

3. Utility Contact:

M. B. KOUDELKA (414) $\sqrt{55-6480}$

4. Licensed Thermal Power (MHt):

5. Nameplate Rating (Gross MHe):

6. Design Electrical Rating (Net MWe):

7. Maximum Dependable Capacity (Gross MWe):

8. Maximum Dependable Capacity (Net MHe):

9. If Changes Occurred Above Since Last Report, Give Reasons:

10. Power Level To Which Restricted, If Any (Net MWe):

11. Reasons For Restrictions, If Any:

\begin{tabular}{|c|c|c|c|}
\hline & MONTH & YEAR & CUMULATIVE \\
\hline 12. Report Period Hrs & 744.0 & $8,760.0$ & $196,513.0$ \\
\hline 13. Hours Reactor Critical & 744.0 & $7,851.0$ & $172,051.6$ \\
\hline 14. Rx Reserve Shtdwn Hrs & 0.0 & 0.0 & 233.9 \\
\hline 15. Hrs Generator On-Line & 744.0 & $7,828.0$ & $169,694.7$ \\
\hline 16. Unit Reserve Shtdwn Hrs & 0.0 & 0.0 & 302.2 \\
\hline 17. Gross Therm Ener (MUH) & $1,115,420.0$ & $11,610,741.0$ & $243,981,925.0$ \\
\hline 18. Gross Elec Ener (МUH) & $377,210.0$ & $3,926,850.0$ & $82,951,110.0$ \\
\hline 19. Net Elec Ener (MUH) & $361,051.0$ & $3,749,875.0$ & $79,080,515.0$ \\
\hline 20. Unit Service factor & 100.0 & 89.4 & 86.4 \\
\hline 21. Unit Avail Factor & 100.0 & 89.4 & 86.5 \\
\hline 22. Unit Cap Factor (MDC Net) & 100.1 & 88.3 & 82.4 \\
\hline 23. Unit Cap Factor (DER Net) & 97.6 & 86.1 & 81.0 \\
\hline 24. Unit Forced Outage Rate & 0.0 & 0.0 & 1.0 \\
\hline 25. Forced Outage Hours & 0.0 & 0.0 & 1.671 .6 \\
\hline
\end{tabular}

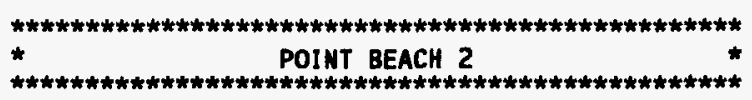

AVERAGE DAILY PONER LEVEL (Net MWe)

\begin{tabular}{ll} 
DAY & PONER \\
\hline 1 & 474 \\
\hline 2 & 487 \\
\hline 3 & 491 \\
\hline 5 & 487 \\
\hline 6 & 483 \\
\hline 7 & 487 \\
\hline 8 & 487 \\
\hline 9 & 486 \\
\hline 10 & 485 \\
\hline 11 & 486 \\
\hline 12 & 486 \\
\hline 13 & 485 \\
\hline 14 & 485 \\
\hline 15 & 484 \\
\hline
\end{tabular}

DAY POWER

\begin{tabular}{ll}
16 & 484 \\
\hline 17 & 483
\end{tabular}

$48 \quad 486$

$19 \quad 484$

485

$21 \quad 486$

$22 \quad 487$

$23 \quad 486$

4886

$25 \quad 486$

$26 \quad 486$

\begin{tabular}{l}
4786 \\
\hline 28
\end{tabular}

\begin{tabular}{ll}
\hline 28 & 487 \\
\hline 29 & 484 \\
\hline 30 & 486 \\
\hline 31 & 485
\end{tabular}

26. Shutdowns Scheduled Over Hext Six Months (Type, Date, Duration):

27. If Currently Shutdown, Estimated Startup Date:

Notes:

DATA REPORTED AND FACTORS CALCULATED AS REQUESTED IN NRC LETTER DATED SEPTEMBER 22, 1977. CUMULATIVE UNIT CAPACITY FACTOR (MDC NET) IS CALCULATED WITH A WEIGHTED AVERAGE. LICENSEE REVISED OCTOBER 1994 NET ELECTRIC ENERGY FROM 0.0 TO $-2,421$.0. 
Report Period DECEMBER 1994

U N I T
A N 0

POUER

REDUCTIONS

$\star$

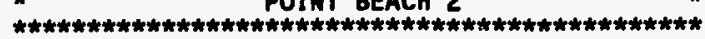

No. Date Type Hours Reason Method LER Number System Component Cause and Corrective Action To Prevent Recurrence

\begin{tabular}{l} 
TYPE \\
\hline F: Forced \\
S: Scheduled
\end{tabular}

F: Forced

\section{REASON}

A-Equipment Failure B-Maintenance or Test C-Refuel ing

D-Regulatory Restriction

-Operator Training \&

License Examination

G-Operational Error

G-Operational Error
H-other

\begin{tabular}{l} 
METHOO \\
\hline 1-Manual \\
2-Manual Scran \\
3-Auto Scram \\
4-Continued \\
5-Reduced Load \\
9-Other
\end{tabular}

5-Reduced Load
9-Other

\section{SYSTEM}

IEEE Standard

NUREG-0161 Exhibit

IEEE Standard

NUREG-0161 


\section{FACILITY DESCRIPTION}

LOCATION

STATE $\ldots \ldots \ldots \ldots \ldots \ldots \ldots \ldots$ HISCONSIH
COUNTY................. MANITONOC

DIST AND DIRECTION FROM

MEAREST POPULATION CTR..... 13 MI NWH OF MANITONOC, HI

TYPE OF REACTOR

PUR

DATE INITIAL CRITICALITY...... MAY 30, 1972

DATE INITIAL ELECTRICITY...... AUGUST 02, 1972

DATE COMMERCIAL OPERATE....... OCTOBER 01, 1972

CONDENSER COOLING METHOD...... ONCE THRU

COMDENSER COOLING WATER....... LAKE MICHIGAN

ELECTRIC RELIABILITY

COUNCIL................... MID-AMERICA INTERPOOL NETWORK
UTILITY

LICENSEE.................. UISCONSIN ELECTRIC POWER CO.

CORPORATE ADDRESS

231 NEST MICHIGAN STREET

MILUAUKEE, WISCONSIN 53201

CONTRACTOR

ARCHITECT/ENGINEER......... BECHTEL

NUC STEAM SYS SUPPLIER...... WESTIMGHOUSE

CONSTRUCTOR.............. BECHTEL

TURBINE SUPPLIER........... VESTINGHOUSE

\section{REGULATORY INFORMATIOW}

IE REGION RESPONSIBLE......... 3

IE RESIDENT IMSPECTOR.

TIMOTHY KOBETZ

LICENSING PROJ MANAGER.

ALLEN G. HANSEN

DOCKET NUMBER

50-301

LICENSE \& DATE ISSUANCE.

DPR 027, MARCH 08, 1973 
1. Docket: $50-282$

OPERATING STATUS

2. Reporting Period: DECEMBER 1994 Outage + On-Line Hrs:

3. Utility Contact: DALE DUGSTAD (612) 388-1121 EXT. 4376

4. Licensed Thermal Power (mit):

5. Nameplate Rating (Gross MWe):

6. Design Electrical Rating (Net MWe):

7. Maximum Dependable Capacity (Gross MWe):

8. Maximum Dependable Capacity (Net MWe):

\begin{tabular}{c}
1650 \\
\hline 593 \\
\hline 530 \\
\hline 545 \\
\hline 513 \\
\hline
\end{tabular}

9. If Changes Occurred Above Since Last Report, Give Reasons:

10. Power Level To thich Restricted, If Any (Wet MHe):

11. Reasons For Restrictions, If Any:

\begin{tabular}{|c|c|c|c|}
\hline & MONTH & YEAR & CUMULATIVE \\
\hline 12. Report Period Hrs & 744.0 & $8,760.0$ & $184,464.0$ \\
\hline 13. Hours Reactor Critical & 744.0 & 7.292 .4 & $157,599.3$ \\
\hline 14. Rx Reserve Shtdun Hrs & 0.0 & 0.0 & $5,571.1$ \\
\hline Hrs Generator On-Line & 744.0 & $7,259.1$ & $155,941.0$ \\
\hline 16. Unit Reserve Shtdun Hrs & 0.0 & 0.0 & 0.0 \\
\hline 17. Gross Therm Ener (MUH) & $1,225,396.0$ & $11,695,491.0$ & $246,908,639.0$ \\
\hline 18. Gross Elec Ener (MUH) & $415,760.0$ & $3,925,580.0$ & $81,349,650.0$ \\
\hline 19. Net Elec Ener (MUH) & $395,846.0$ & $3,715,483.0$ & $76,455,267.0$ \\
\hline 20. Unit Service Factor & 100.0 & 82.9 & 84.5 \\
\hline 21. Unit Avail Factor & 100.0 & 82.9 & 84.5 \\
\hline 22. Unit Cap Factor (MDC Net) & 103.7 & 82.7 & 82.3 \\
\hline 23. Unit Cap Factor (DER Net) & 100.4 & 80.0 & 78.2 \\
\hline 24. Unit Forced Outage Rate & 0.0 & 2.2 & 5.0 \\
\hline Forced Outage Hours & 0.0 & 165.3 & $8,125.9$ \\
\hline
\end{tabular}

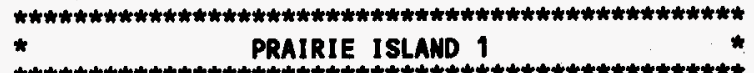

AVERAGe DAILY PONER LEVEL (Net MUNe)

\begin{tabular}{ll} 
DAY & PONER \\
\hline 1 & 532 \\
\hline 2 & 532 \\
\hline 3 & 532 \\
\hline 4 & 532 \\
\hline 5 & 532 \\
\hline 6 & 532 \\
\hline 7 & 531 \\
\hline 8 & 533 \\
\hline 9 & 531 \\
\hline 10 & 533 \\
\hline 11 & 535 \\
\hline 12 & 528 \\
\hline 13 & 531 \\
\hline 14 & 531 \\
\hline 15 & 532 \\
\hline
\end{tabular}

\begin{tabular}{ll} 
DAY & PONER \\
\hline 16 & 532 \\
\hline 17 & 533 \\
\hline 18 & 532 \\
\hline 19 & 532 \\
\hline 20 & 532 \\
\hline 21 & 533 \\
\hline 22 & 534 \\
\hline 23 & 531 \\
\hline 24 & 532 \\
\hline 25 & 531 \\
\hline 26 & 532 \\
\hline 27 & 533 \\
\hline 28 & 532 \\
\hline 29 & 534 \\
\hline 30 & 531 \\
\hline 31 & 532 \\
\hline
\end{tabular}

26. Shutdowns Scheduled Over Mext six Months (Type, Date, Duration):

27. If Currently Shutdown, Estimated Startup Date:

Notes:

CUMULATIVE UNIT CAPACITY FACTOR (MDC NET) IS CALCULATED WITH A MEIGHTED AVERAGE. 
TYPE

F: Forced

S: Scheduled

\section{REASON}

A-Equipment Faílure B-Maintenance or Test -Refuel ing

D-Regulatory Restriction

E-operator Training \&

F-Administrative

G-Operational Error

H-Other

\section{METHOO}

1-Manual

2-Manual Scram

3-Auto Scram

3-Auto Scram

5-Reduced Load

9-other

\section{SYSTEM}

IEEE Standard

805-1984 and/or

NUREG-0161 Exhibit F
COMPONENT

IEEE Standard

803A-1983 and/or

NUREG-0161 Exhibit H 


\section{FACILITY DESCRIPTION}

\section{LOCATION}

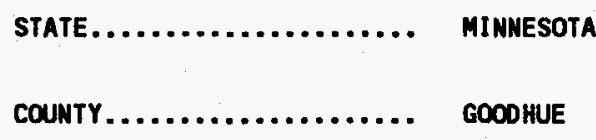

\section{OIST AND DIRECTION FRON}

MEAREST POPULATION CTR..... 28 MI SE OF MINMEAPOLIS, MN

TYPE OF REACTOR.

PUR

DATE INITIAL CRITICALITY...... DECEMBER 01, 1973

DATE INITIAL ELECTRICITY...... DECEMBER 04, 1973

Date COMmercial OPERATE....... December 16, 1973

CONDENSER COOLING METHOD...... COOLING TONERS

CONDENSER COOLING WATER....... MISSISSIPPI RIVER

ELECTRIC RELIABILITY

COUNCIL.................... MID-CONTINENT AREA RELIABILITY

\section{UTILITY \& CONTRACTOR INFORMATION}

UTILITY

LICENSEE.................. MORTHERN STATES POMER CO.

CORPORATE ADDRESS........ 414 NICOLLET MALL

MINNEAPOLIS, MINNESOTA 55401

\section{CONTRACTOR}

ARCHI TECT/ENGINEER......... FLUOR PIONEER, INC.

MUC STEAM SYS SUPPLIER...... WESTINGHOUSE

CONSTRUCTOR.

MORTHERN STATES PONER COMPANY

TURBINE SUPPLIER.

WESTIMGHOUSE

\section{REGULATORY INFORMATION}

IE REGION RESPONSIBLE......... 3

IE RESIDENT INSPECTOR......... MARC DAPAS

LICENSING PROJ MANAGER......... MARSHA GAMBERONI

DOCKET MUMBER.............. 50-282

LICENSE \& DATE ISSUANCE......... DPR 042, APRIL 05, 1974 
1. Docket: $50-306$

OPERATING STATUS

2. Reporting Period: DECEMBER 1994

Outage + On-Line Hrs: $\quad 744.0$

3. Utility Contact: DALE DUGSTAD (612) 388-1121 EXT. 4376

4. Licensed Thermal Power (MUt):

5. Nameplate Rating (Gross WWe):

6. Design Electrical Rating (Net MUe):

7. Maximum Dependable Capacity (Gross MWe):

8. Maximum Dependable Capacity (Net MHe):

\begin{tabular}{c}
1650 \\
\hline 593 \\
\hline 530 \\
\hline 544 \\
\hline 512
\end{tabular}

9. If Changes Occurred Above Since Last Report, Give Reasons:

10. Power Level To Which Restricted, If Any (Net MWe):

11. Reasons for Restrictions, If Any:

\begin{tabular}{|c|c|c|c|}
\hline & MONTH & YEAR & CUAULATIVE \\
\hline 12. Report Period Hrs & 744.0 & $8,760.0$ & $175,582.0$ \\
\hline 13. Hours Reactor Critical & 744.0 & $8,743.2$ & $155,110.2$ \\
\hline 14. Rx Reserve Shtdhn Hrs & 0.0 & 0.0 & $1,516.1$ \\
\hline 15. Hrs Generator On-Line & 744.0 & $8,734.3$ & $153,722.7$ \\
\hline 16. Unit Reserve Shtdkm Mrs & 0.0 & 0.0 & 0.0 \\
\hline 17. Gross Therm Ener (MWH) & $1,206,313.0$ & $14,324,583.0$ & $244,280,222.0$ \\
\hline 18. Gross Elec Ener (MUH) & $408,690.0$ & $4,806,870.0$ & $79,712,040.0$ \\
\hline 19. Net Elec Ener (МWH) & $389,070.0$ & $4,552,960.0$ & $75,357,496.0$ \\
\hline 20. Unit Service Factor & 100.0 & 99.7 & 87.6 \\
\hline 21. Unit Avail Factor & 100.0 & 99.7 & 87.6 \\
\hline 22. Unit Cap Factor (MDC Net) & 102.1 & 101.5 & 85.7 \\
\hline 23. Unit Cap Factor (DER Net) & 98.7 & 98.1 & 81.0 \\
\hline 24. Unit Forced Outage Rate & 0.0 & 0.2 & 2.5 \\
\hline 25. Forced Outage Hours & 0.0 & 20.7 & $4,010.7$ \\
\hline
\end{tabular}

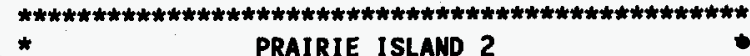
* PRAIRIE ISLAND 2 .

AVERAGE DAILY POUER LEVEL (Net MNe)

\begin{tabular}{ll} 
DAY & PONER \\
\hline 1 & 533 \\
\hline 2 & 531 \\
\hline 3 & 533 \\
\hline 4 & 531 \\
\hline 5 & 534 \\
\hline 6 & 533 \\
\hline 7 & 532 \\
\hline 8 & 533 \\
\hline 9 & 533 \\
\hline 10 & 260 \\
\hline 11 & 531 \\
\hline 12 & 531 \\
\hline 13 & 531 \\
\hline 14 & 532 \\
\hline 15 & 532 \\
\hline
\end{tabular}

\begin{tabular}{ll} 
DAY & PONER \\
\hline 16 & 531 \\
\hline 17 & 532 \\
\hline 18 & 531 \\
\hline 19 & 532 \\
\hline 20 & 532 \\
\hline 21 & 532 \\
\hline 22 & 534 \\
\hline 23 & 531 \\
\hline 24 & 531 \\
\hline 25 & 531 \\
\hline 26 & 531 \\
\hline 27 & 531 \\
\hline 28 & 531 \\
\hline 29 & 531 \\
\hline 30 & 533 \\
\hline 31 & 531 \\
\hline
\end{tabular}

26. Shutdowns scheduled Over Next Six Months (Type, Date, Duration): REFUELING OUTAGE, MAY 1995.

27. If Currently Shutdown, Estimated Startup Date: Notes:

CUMULATIVE UNIT CAPACITY FACTOR (MDC NET) IS CALCULATED WITH A WEIGHTED AVERAGE. 


\begin{tabular}{|c|c|c|c|c|c|c|c|c|c|}
\hline No. & Date & Type & Hours & Reason & Method & LER Number & System & Component & Cause and Corrective Action To Prevent Recurrence \\
\hline & $12 / 10 / 94$ & $\mathbf{s}$ & 0.0 & B & 5 & & & & $\begin{array}{l}\text { TURBINE VALVE TESTING AND CONDENSER WATERBOX } \\
\text { CLEANING. }\end{array}$ \\
\hline
\end{tabular}

Cause and Corrective Action To Prevent Recurrence

\begin{tabular}{|c|c|}
\hline TYPE & REASON \\
\hline $\begin{array}{l}\text { F: Forced } \\
\text { S: Scheduled }\end{array}$ & $\begin{array}{l}\text { A-Equipment Fail lure } \\
\text { B-Maintenance or Test } \\
\text { C-Refuel ing } \\
\text { D-Regulatory Restriction } \\
\text { E-Operator Training \& } \\
\text { License Examination } \\
\text { F-Administrat ive } \\
\text { G-Operat ional Error } \\
\text { H-Other }\end{array}$ \\
\hline
\end{tabular}

\begin{tabular}{l} 
METHOD \\
\hline 1-Manual \\
2-Manual Scram \\
3-Auto Scram \\
4-Cont inued \\
5-Reduced Load \\
9-0ther
\end{tabular}

\section{SYSTEM}

IEEE Standard

NUREG-0161 Exhibit F

\section{COMPONENT}

\section{IEEE Standard}

1983 and/or

NUREG-0161 Exhibit H

F-Administrat ive

$H$-other 
FACILITY DESCRIPTION

LOCATION

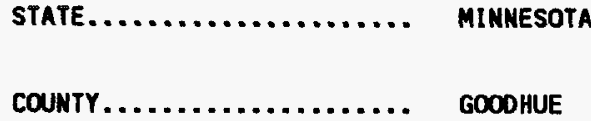

DIST AND DIRECTION FROM MEAREST POPULATION CTR..... 28 MI SE OF MINNEAPOLIS, MH

TYPE OF REACTOR PUR

DATE Initial CRIticalitY...... DeCember 17, 1974

DATE INITIAL ELECTRICITY...... DeCEMBER 21, 1974

DATE COMMERCIAL OPERATE....... DeCEMBER 21, 1974

CONDENSER COOLING METHOD...... COOLING TONERS

CONDENSER COOLING WATER....... MISSISSIPPI RIVER

ELECTRIC RELIABILITY

COUNCIL................... MID-CONTINENT AREA RELIABILITY

\section{UTILITY \& CONTRACTOR INFORMATION}

UTILITY

LICENSEE................ NORTHERN STATES PONER CO.

CORPORATE ADDRESS.......... 414 NICOLLET MALL

MINNEAPOLIS, MINNESOTA 55401

\section{CONTRACTOR}

ARCHI TECT/ENGIMEER......... FLUOR PIONEER, INC.

NUC STEAM SYS SUPPLIER...... WESTIMGHOUSE

CONSTRUCTOR

MORTHERN STATES PONER COMPANY

TURBINE SUPPLIER.

WEST INGHOUSE

\section{REGULATORY INFORMATION}

IE REGION RESPONSIBLE......... 3

IE RESIDENT INSPECTOR

MARC DAPAS

LICENSING PROJ MANAGER

MARSHA GAMBERONI

DOCKET NUMBER

$50-306$

LICENSE \& DATE ISSUANCE. 
1. Docket: $50-254$

OPERATING STATUS

2. Reporting Period: DECEMBER 1994

Outage + On-Line Hrs: $\quad 744.0$

3. Utility Contact: KRISTAL MOORE (309) 654-2241 EXT. 3070

4. Licensed Thermal Power (MWt):

5. Nameplate Rating (Gross Mue):

6. Design Electrical Rating (Net MHe):

7. Maximum Dependable Capacity (Gross MWe):

8. Maximum Dependable Capacity (Net MWe):

$\begin{array}{r}2511 \\ \hline 828 \\ \hline 789 \\ \hline 813 \\ \hline 769\end{array}$

9. If Changes Occurred Above Since Last Report, Give Reasons:

10. Power Level To Which Restricted, If Any (Net MHe):

11. Reasons for Restrictions, If Any:

\begin{tabular}{|c|c|c|c|}
\hline & MONTH & YEAR & CUMULAT IVE \\
\hline 12. Report Period Hrs & 744.0 & $8,760.0$ & $199,175.0$ \\
\hline 13. Hours Reactor Critical & 0.0 & $2,651.2$ & $152,433.5$ \\
\hline 14. Rx Reserve Shtdun Hrs & 0.0 & 0.0 & $3,421.9$ \\
\hline 15. Hrs Generator on-Line & 0.0 & $2,527.6$ & $147,822.9$ \\
\hline 16. Unit Reserve Shtdwn Hrs & 0.0 & 0.0 & 909.2 \\
\hline 17. Gross Therm Ener (MHH) & 0.0 & $5,462,936.2$ & $319,156,124.8$ \\
\hline 18. Gross Elec Ener (MUH) & 0.0 & $1,765,735.0$ & $103,463,854.0$ \\
\hline 19. Net Elec Ener (MUH) & 0.0 & $1,670,168.0$ & $97,588,166.0$ \\
\hline 20. Unit Service Factor & 0.0 & 28.9 & 74.2 \\
\hline 21. Unit Avail Factor & 0.0 & 28.9 & 74.7 \\
\hline 22. Unit Cap Factor (MDC Net) & 0.0 & 24.8 & 63.7 \\
\hline 23. Unit Cap Factor (DER Net) & 0.0 & 24.2 & 62.1 \\
\hline 24. Unit Forced Outage Rate & 100.0 & $\overline{46.4}$ & 7.5 \\
\hline 25. Forced Outage Hours & 744.0 & 2190.9 & $11,964.8$ \\
\hline
\end{tabular}

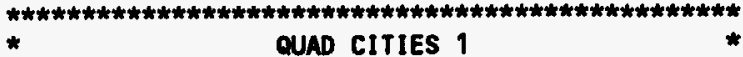

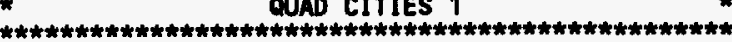

AVERAGe DAILY PONER LEVEL (Net MHe)

\begin{tabular}{lc} 
DAY & PONER \\
\hline 1 & -9 \\
\hline 2 & -9 \\
\hline 3 & -9 \\
\hline 4 & -9 \\
\hline 5 & -9 \\
\hline 6 & -9 \\
\hline 7 & -9 \\
\hline 8 & -7 \\
\hline 9 & -8 \\
\hline 10 & -9 \\
\hline 11 & -8 \\
\hline 12 & -9 \\
\hline 13 & -9 \\
\hline 14 & -9 \\
\hline 15 & -10 \\
\hline
\end{tabular}

\begin{tabular}{ll} 
DAY & PONER \\
\hline 16 & -12 \\
\hline 17 & -10 \\
\hline 18 & -9 \\
\hline 19 & -9 \\
\hline 20 & -9 \\
\hline 21 & -9 \\
\hline 22 & -9 \\
\hline 23 & -9 \\
\hline 24 & -8 \\
\hline 25 & -8 \\
\hline 26 & -8 \\
\hline 27 & -8 \\
\hline 28 & -8 \\
\hline 29 & -8 \\
\hline 30 & -8 \\
\hline 31 & -8 \\
\hline
\end{tabular}

26. Shutdowns Scheduled Over Mext Six Months (Type, Date, Duration):

27. If Currently Shutdown, Estimated Startup Date: 01/13/95 Notes: 
Report Period DECEMBER 1994

\section{No.}

Date

Type

Hours

SHUTOOWHS

A N D

POWER REDUCTIONS

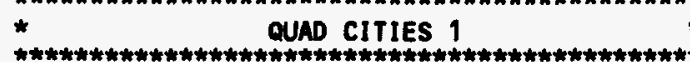
Cause and Corrective Action To Prevent Recurrence

Method

LER Number

System

Component

10/02/94

744.0

CONTINUED FORCED OUTAGE.

94-005 $\mathbf{F}$ G

4

94015

TYPE

\section{F: Forced}

s: Scheduled

\section{REASON}

A-Equipment Fai lure B-Maintenance or Test C-Refueling

D-Regulatory Restriction

- Operator Training 8

License Examinat

G-Operational Error

H-Other

\section{SYSTEM}

IEEE Standard

1-Manual

3-Muto Scram

4-Cont inued

5-Reduced Load

9-0ther

\section{COMPONENT}

IEEE Standard

MUREG-0161 Exhibit 


\section{FACILITY DESCRIPTION}

\section{LOCATION}

STATE.

COUNTY

LLINOIS

ROCK ISLAND

\section{DIST AND DIRECTION FRON} MEAREST POPULATION CTR....

20 MI ME OF MOLINE, IL

TYPE OF REACTOR

DATE INITIAL CRITICALITY...... OCTOBER 18, 1971

DATE INITIAL ELECTRICITY...... APRIL 12, 1972

DATE COMmERCIAL OPERATE....... FEbruARY 18, 1973

CONDENSER COOLING METHOD...... O ONCE THRU

CONDENSER COOLIMG MATER....... MISSISSIPPI RIVER

ELECTRIC RELIABILITY

COUNCIL...............
UTILITY \& CONTRACTOR INFORMATION

UTILITY

LICENSEE................. COMMONUEALTH EdisON CO.

CORPORATE ADDRESS.

1400 OPUS PL., OPUS WEST III SUITE 300

DOWWER'S GROVE, ILLIMOIS 60515

\section{CONTRACTOR}

ARCHI TECT/ENGINEER

SARGENT \& LUNDY

NUC STEAM SYS SUPPLIER

GeNERAL ELECTRIC

CONSTRUCTOR

UNITED ENG. \& CONSTRUCTORS

TURBINE SUPPLIER

GENERAL ELECTRIC

\section{REGULATORY INFORMATION}

IE REGION RESPONSIBLE.

3

IE RESIDENT INSPECTOR

CHRISTOPHER MILLER

LICENSING PROJ MANAGER.

ROBERT M. PULSI FER

DOCKET NUMBER.

50-254

LICENSE \& DATE ISSUANCE.

DPR 029, DECEMBER 14, 1972 
1. Docket: $50-265$

OPERATING STATUS

2. Reporting Period: DECEMBER 1994

Outage + On-line Hrs: $\mathbf{7 4 4 . 0}$

3. Utility Contact: KRISTAL MOORE (309) 654-2241 EXT. 3070

4. Licensed Thermal Power (MWt):

5. Nameplate Rating (Gross MWe):

6. Design Electrical Rating (Net MHe):

7. Maximum Dependable Capacity (Gross MMe):

8. Maximum Dependable Capacity (Net MWe):

9. If Changes Occurred Above Since Last Report, Give Reasons:

10. Power Level To Which Restricted, If Any (Net MWe):

11. Reasons for Restrictions, If Any:

\begin{tabular}{|c|c|c|c|}
\hline & MONTH & YEAR & CUMULATIVE \\
\hline 12. Report Period Hrs & 744.0 & $8,760.0$ & $197,612.0$ \\
\hline 13. Hours Reactor Critical & 402.2 & $5,874.2$ & $149,776.3$ \\
\hline 14. Rx Reserve Shtdwn Hrs & 0.0 & 0.0 & $2,985.8$ \\
\hline 15. Hrs Generator On-Line & 353.3 & $5,748.5$ & $145,931.7$ \\
\hline 16. Unit Reserve Shtdun Hrs & 0.0 & 0.0 & 702.9 \\
\hline 17. Gross Therm Ener (MUH) & $397,065.6$ & $13,159,742.2$ & $315,940,704.9$ \\
\hline 18. Gross Elec Ener (MUH) & $115,449.0$ & $4,204,131.0$ & $101,513,901.0$ \\
\hline 19. Net Elec Ener (MWH) & $104,905.0$ & $4,013,349.0$ & $96,155,909.0$ \\
\hline 20. Unit Service Factor & 47.5 & 65.6 & 73.8 \\
\hline 21. Unit Avail Factor & 47.5 & 65.6 & 74.2 \\
\hline 22. Unit Cap Factor (MDC Net) & 18.3 & 59.6 & 63.3 \\
\hline 23. Unit Cap Factor (DER Net) & 17.9 & 58.1 & 61.7 \\
\hline 24. Unit Forced Outage Rate & 52.5 & 34.3 & 9.9 \\
\hline 25. Forced Outage Hours & 390.7 & 3000.3 & $16,078.1$ \\
\hline
\end{tabular}

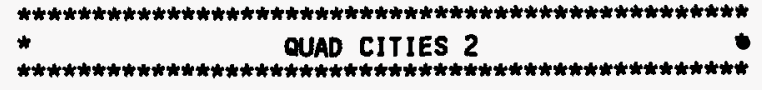

AVERAGE DAILY PONER LeVEL (Net Me)

\begin{tabular}{ll} 
DAY & PONER \\
\hline 1 & -8 \\
\hline 2 & -9 \\
\hline 4 & -8 \\
\hline 5 & -8 \\
\hline 6 & -8 \\
\hline 7 & -8 \\
\hline 8 & -8 \\
\hline 9 & -6 \\
\hline 10 & -7 \\
\hline 11 & -8 \\
\hline 12 & -8 \\
\hline 13 & -8 \\
\hline 14 & -8 \\
\hline 15 & -9 \\
\hline
\end{tabular}

\begin{tabular}{cc} 
DAY & PONER \\
\hline 16 & -11 \\
\hline 17 & 33 \\
\hline 18 & 121 \\
\hline 19 & 142 \\
\hline 20 & 173 \\
\hline 21 & 166 \\
\hline 22 & 167 \\
\hline 23 & 175 \\
\hline 24 & 246 \\
\hline 25 & 272 \\
\hline 26 & 272 \\
\hline 27 & 272 \\
\hline 28 & 596 \\
\hline 29 & 645 \\
\hline 30 & 614 \\
\hline 31 & 612 \\
\hline
\end{tabular}

26. Shutdowns Scheduled Over Next Six Months (Type, Date, Duration):

27. If Currently Shutdown, Estimated Startup Date:

Notes: 
UNIT SHUTDOHNS AND POWER REDUCTIONS

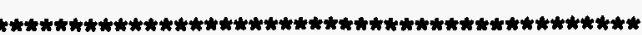
MTt SHUTDOHNS A

\begin{tabular}{cccccc}
\hline No. & Date & Type & Hours & Reason & Method \\
\hline $94-15$ & $10 / 03 / 94$ & F & 390.7 & F & 4 \\
$94-16$ & $12 / 29 / 94$ & F & 0.0 & B & 5
\end{tabular}

LER Number

System

Component

Cause and Corrective Action To Prevent Recurrence

CONTIMUED FORCED OUTAGE.

LOAD DROP TO ACCOMMODATE CONDENSATE DEMIMERALIZER BACKWASH AND PRECOAT.

\begin{tabular}{|c|c|}
\hline TYPE & REASON \\
\hline $\begin{array}{l}\text { F: Forced } \\
\text { S: Scheduled }\end{array}$ & $\begin{array}{l}\text { A-Equipment Failure } \\
\text { B-Maintenance or Test } \\
\text { C-Refuel ing } \\
\text { D-Regulatory Restriction } \\
\text { E-Operator Training \& } \\
\text { License Examination } \\
\text { F-Acministrative } \\
\text { G-Operational Error } \\
\text { H-Other }\end{array}$ \\
\hline
\end{tabular}

\begin{tabular}{l} 
METHOD \\
\hline 1-Manual \\
2-Manual Scram \\
3-Auto Scram \\
4-Cont inued \\
5-Reduced Load \\
9-0ther
\end{tabular}

\begin{tabular}{l} 
SYSTEM \\
\hline IEEE Standard \\
805-1984 and/or \\
NUREG-0161 Exhibit F
\end{tabular}

COMPONENT

IEEE Standard

803A-1983 and/or

NUREG-0161 Exhibit H

G-Operational Error 


\section{FACILITY DESCRIPTION}

LOCATION

STATE

COUNTY

DIST AND DIRECTION FRON

MEAREST POPULATION CTR

TYPE OF REACTOR.

DATE INITIAL CRITICALITY...... APRIL 26, 1972

DATE INITIAL ELECTRICITY....... MAY 23, 1972

DATE COMMERCIAL OPERATE........ MARCH 10, 1973

CONDENSER COOLING METHOD....... ONCE THRU

CONDENSER COOLING MATER....... MISSISSIPPI RIVER

ELECTRIC RELIABILITY

COUNCIL.................. MID-AMERICA INTERPOOL METHORK

\section{UTILITY \& CONTRACTOR INFORMATION}

\section{UTILITY}

LICENSEE.

CORPORATE ADDRESS.

COMHONUEALTH EDISON CO.

1400 OPUS PL., OPUS HEST III

SUITE 300

DOWER'S GROVE, ILLINOIS 60515

CONTRACTOR

ARCHITECT/ENGINEER......... SARGENT \& LUNDY

NUC STEAM SYS SUPPLIER...... GENERAL ELECTRIC

CONSTRUCTOR ............... UNITED ENG \& COUSTRUCTORS

TURBINE SUPPLIER.......... GENERAL ELECTRIC

\section{REGULATORY INFORMATION}

IE REGION RESPONSIBLE........ 3

IE RESIDENT INSPECTOR. CHRISTOPHER MILLER

LICENSING PROJ MANAGER. ROBERT M. PULSIFER

DOCKET NUMBER

$50-265$

LICENSE \& DATE ISSUANCE.

DPR 030, DECEMBER 14, 1972 
1. Docket: $50-458$

OPERATING STATUS

2. Reporting Period: DECEMBER 1994

Outage + On-Line Hrs: 744.0

3. Utility Contact: D. R. GLUECK (504) 381-4317

4. Licensed Thermal Power (mut):

5. Nameplate Rating (Gross MUe):

6. Design Electrical Rating (Net WWe):

7. Maximum Dependable Capacity (Gross MHe):

8. Maximum Dependable Capacity (Net MWe):

9. If Changes Occurred Above Since Last Report, Give Reasons:

10. Power Level To Which Restricted, If Any (Net MWe):

11. Reasons For Restrictions, If Any:

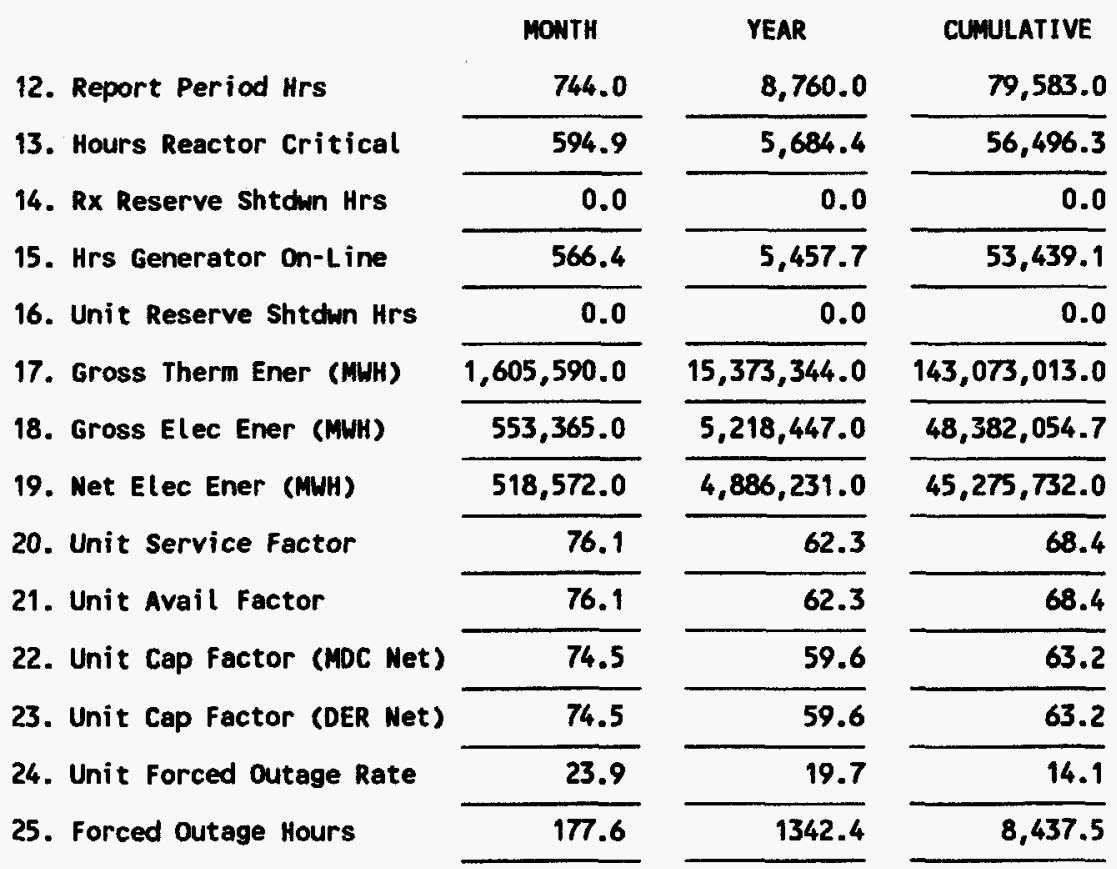

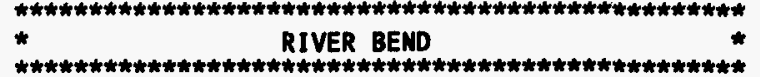

AVERAGe dAILY POWER LeVEL (Net MHe)

\begin{tabular}{lr} 
DAY & POWER \\
\hline 1 & 941 \\
\hline 2 & 935 \\
\hline 3 & 932 \\
\hline 4 & 777 \\
\hline 5 & 0 \\
\hline 6 & 0 \\
\hline 7 & 0 \\
\hline 8 & 0 \\
\hline 9 & 0 \\
\hline 10 & 0 \\
\hline 11 & 0 \\
\hline 12 & 294 \\
\hline 13 & 888 \\
\hline 14 & 940 \\
\hline 15 & 937 \\
\hline
\end{tabular}

\begin{tabular}{cc}
\hline DAY & PONER \\
\hline 16 & 928 \\
\hline 17 & 924 \\
\hline 18 & 942 \\
\hline 19 & 941 \\
\hline 20 & 938 \\
\hline 21 & 937 \\
\hline 22 & 939 \\
\hline 23 & 937 \\
\hline 24 & 941 \\
\hline 25 & 941 \\
\hline 26 & 941 \\
\hline 27 & 940 \\
\hline 28 & 937 \\
\hline 29 & 936 \\
\hline 30 & 929 \\
\hline 31 & 914 \\
\hline
\end{tabular}

26. Shutdowns Scheduled Over Next Six Months (Type, Date, Duration):

27. If Currently Shutdown, Estimated Startup Date:

Notes:

CUMULATIVE VALUES FOR ITEMS 12, 13, 15 AMD 17-19 ARE CALCULATEd USING PRE-COMNERCIAL DATA, WHILE CLMULATIVE VALUES FOR ITEMS 2025 ARE CALCULATED SINCE COMMERCIAL OPERATION. 


\begin{tabular}{|c|c|c|c|c|c|c|c|c|c|}
\hline No. & Date & Type & Hours & Reason & Method & LER Number & System & Component & Cause and Corrective Action To Prevent Recurrence \\
\hline $94-03$ & $12 / 04 / 94$ & $\mathbf{F}$ & 177.6 & $H$ & 3 & & & & $\begin{array}{l}\text { AN AUTOMATIC SHUTDOWN OCCURRED UHEN THE ISOLATION } \\
\text { LOGIC WAS SATISFIED AND A FULL MSIV ISOLATION } \\
\text { OCCURRED DURING THE PERFORMANCE OF STP-058-4501, } \\
\text { CONTAINMENT AND DRYWELL MANUAL ISOLATION } \\
\text { ACTUATION CHANMEL FUNCTIONAL TEST. }\end{array}$ \\
\hline
\end{tabular}

LOGIC WAS SATISFIED AND A FULL MSIV ISOLATION

CONTAINMENT AND DRYWELL MANUAL ISOLATION

ACTUATION CHANMEL FUNCTIONAL. TEST.

\begin{tabular}{ll} 
TYPE & REASON \\
\hline F: Forced & $\begin{array}{l}\text { A-Equipment Failure } \\
\text { B-Maintenance or Test } \\
\text { C-Refuel ing } \\
\text { D-Regulatory Restriction } \\
\text { E-Operator Training \& } \\
\text { License Examination } \\
\text { F-Administrative } \\
\text { G-Operational Error } \\
\text { H-Other }\end{array}$
\end{tabular}

\begin{tabular}{l} 
METHCD \\
\hline 1-Manual \\
2-Manual Scram \\
3-Auto Scram \\
4-Cont inued \\
5-Reduced Load \\
9-0ther
\end{tabular}

\section{SYSTEM}

IEEE Standard

805-1984 and/or

NUREG-0161 Exhibit F

\section{COMPONENT}

IEEE Standard

803A-1983 and/or

NUREG-0161 Exhibit H 


\section{FACILITY DESCRIPTION}

\section{LOCATION}

$$
\text { STATE. }
$$

COUNTY

\section{DIST ANO DIRECTION FROM}

CTR.

TYPE OF REACTOR.

LOUISIANA

WEST FELICIANA

24 MI NNH OF BATON ROUGE, LA

BWR

DATE INITIAL CRITICALITY...... OCTOBER 31, 1985

DATE INITIAL ELECTRICITY....... DECEMBER 03, 1985

DATE COMmERcial operate....... JUNE 16, 1986

CONDENSER COOLING METHOD...... MDCT

CONDENSER COOLING WATER...... MISSISSIPPI RIVER

ELECTRIC RELIABILITY

COUNCIL.................... SOUTHWEST POWER POOL

\section{UTILITY \& CONTRACTOR INFORMATION}

UTILITY

LICENSEE.

CORPORATE ADDRESS.

\section{CONTRACTOR}

ARCHITECT/ENGINEER

MUC STEAM SYS SUPPLIER......

CONSTRUCTOR.

TURBIME SUPPLIER

ENTERGY OPERATIONS, INC.

P.0. BOX 220

ST. FRANCISVILLE LOUISIANA T7705

\section{REGULATORY IMFORMATION}

IE REGION RESPONSIBLE

IE RESIDENT INSPECTOR

WARD SMITH

LICENSING PROJ MANAGER.

RAMON V. AZUA

DOCKET NUMABR.

$50-458$

LICENSE \& DATE ISSUANCE.
STONE \& WEBSTER

GENERAL ELECTRIC

STONE \& WEBSTER

GEMERAL ELECTRIC 
1. Docket: $50-261$

OPERATING STATUS

2. Reporting Period: DECEMBER 1994

Outage + On-Line Hrs: 744.0

3. Utility Contact: J. S. SCARBOROUGH (803) 857-1000

4. Licensed Thermal Power (MUt):

5. Nameplate Rating (Gross MWe):

6. Design Electrical Rating (Net MWe):

7. Maximum Dependable Capacity (Gross MUe):

8. Maximum Dependable Capacity (Net MWe):

9. If Changes Occurred Above Since Last Report, Give Reasons:

10. Power Level To Which Restricted, If Any (Net Mwe):

11. Reasons For Restrictions, If Any:

\begin{tabular}{|c|c|c|c|}
\hline & MONTH & YEAR & CUMULATIVE \\
\hline 12. Report Period Hrs & 744.0 & $8,760.0$ & $208,968.0$ \\
\hline 13. Hours Reactor Critical & 744.0 & $6,963.6$ & $147,411.5$ \\
\hline 14. Rx Reserve Shtdwn Hrs & 0.0 & 0.0 & $3,314.7$ \\
\hline 15. Hrs Generator on-Line & 744.0 & $6,847.2$ & $144,319.2$ \\
\hline 16. Unit Reserve Shtdin Hrs & 0.0 & 0.0 & 23.2 \\
\hline 17. Gross Therm Ener (MUH) & $1,697,449.7$ & $15,185,845.7$ & $295,495,320.0$ \\
\hline 18. Gross Elec Ener (MHH) & $561,971.0$ & $4,908,541.0$ & $95,862,546.0$ \\
\hline 19. Net Elec Ener (MUH) & $536,120.0$ & $4,646,412.0$ & $90,579,351.0$ \\
\hline 20. Unit Service Factor & 100.0 & 78.2 & 69.1 \\
\hline 21. Unit Avail Factor & 100.0 & 78.2 & 69.1 \\
\hline 22. Unit Cap Factor (MDC Net) & 105.5 & 77.7 & 63.5 \\
\hline 23. Unit Cap Factor (DER Net) & 102.9 & 75.8 & 61.9 \\
\hline 24. Unit Forced Outage Rate & 0.0 & 21.8 & 15.2 \\
\hline 25. Forced Outage Hours & 0.0 & 1912.8 & $25,843.7$ \\
\hline
\end{tabular}

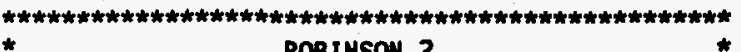

* ROB INSON 2 .

AVERAGE DAILY POWER LEVEL (Net MHe)

\begin{tabular}{ll} 
DAY & POWER \\
\hline 1 & 723 \\
\hline 2 & 722 \\
\hline 3 & 723 \\
\hline 4 & 722 \\
\hline 5 & 722 \\
\hline 6 & 721 \\
\hline 7 & 720 \\
\hline 8 & 720 \\
\hline 9 & 718 \\
\hline 10 & 719 \\
\hline 11 & 720 \\
\hline 12 & 722 \\
\hline 13 & 722 \\
\hline 14 & 724 \\
\hline 15 & 725 \\
\hline
\end{tabular}

\begin{tabular}{ll} 
DAY & PONER \\
\hline 16 & 724 \\
\hline 17 & 720 \\
\hline 18 & 637 \\
\hline 19 & 726 \\
\hline 20 & 726 \\
\hline 21 & 726 \\
\hline 22 & 726 \\
\hline 23 & 726 \\
\hline 24 & 727 \\
\hline 25 & 727 \\
\hline 26 & 726 \\
\hline 27 & 725 \\
\hline 28 & 726 \\
\hline 29 & 726 \\
\hline 30 & 724 \\
\hline 31 & 724 \\
\hline
\end{tabular}

26. Shutdowns Scheduled Over Next Six Months (Type, Date, Duration): REFUELING OUTAGE, APRIL 29, 1995, 39 DAYS.

27. If Currently Shutdown, Estimated Startup Date:

Notes: 


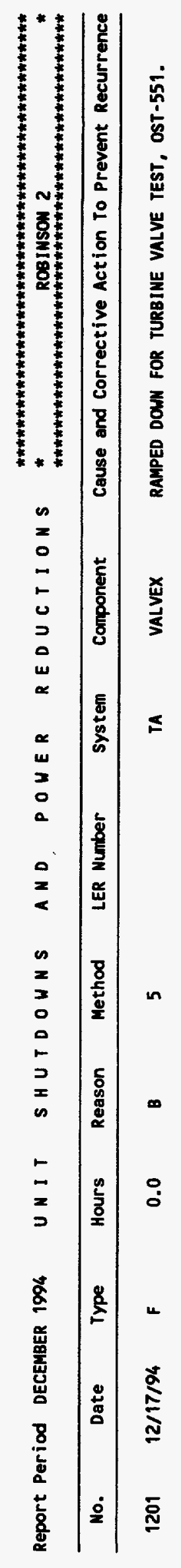

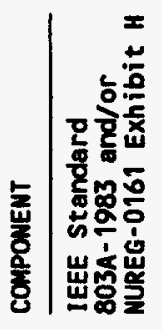

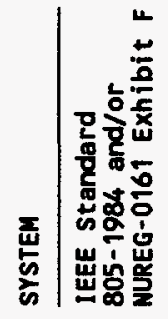

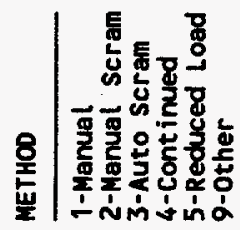

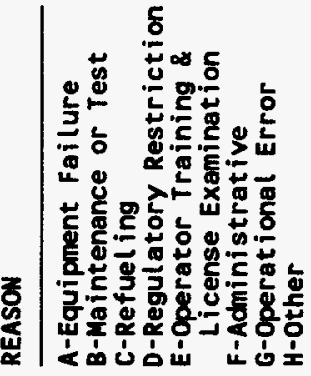

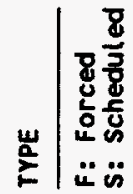




\section{*}

FACILITY DESCRIPTION

\section{LOCATION}

COUNTY.

DARLINGTON

\section{DIST AND DIRECTION FROM}

MEAPEST POPULATION CTR.

DATE INITIAL CRITICALITY...... SEPTEMBER 20, 1970

DATE INITIAL ELECTRICITY...... SEPTEMBER 26, 1970

DATE COMMERCIAL OPERATE....... MARCH 07, 1971

CONDENSER COOLING METHOD...... RECIRCULATION

CONDENSER COOLING WATER....... ROBINSON IMPOUNDMENT

ELECTRIC RELIABILITY

COUNCIL................... SOUTHEASTERN ELECTRIC RELIABILITY COUNCIL

\section{UTILITY \& CONTRACTOR INFORMATION}

\section{UTILITY}

LICENSEE

CORPORATE ADDRESS.

CAROLINA PONER \& LIGHT CO.

P.0. BOX 1551

RALEIGH, NORTH CAROLINA 27602

CONTRACTOR

ARCHITECT/ENGINEER.......... EBASCO

MUC STEAM SYS SUPPLIER....... MESTIMGHOUSE

CONSTRUCTOR............... EBASCO

TURBINE SUPPLIER.......... WESTINGHOUSE

\section{REGULATORY INFORMATIOH}

IE REGION RESPOWSIBLE......... 2

IE RESIDENT IMSPECTOR......... WILLIAM ORDERS

LICENSING PROJ MANAGER......... BRENDA L. MOZAFARI

DOCKET NUMBER.............. 50-261

LICENSE \& DATE ISSUANCE........ DPR 023, SEPTEMBER 23, 1970 
1. Docket: $50-272$

OPERATING STATUS

2. Reporting Period: DECEMBER 1994

Outage + On-Line Hrs: $\mathbf{7 4 4 . 0}$

3. Utility Contact: MIKE MORRONI (609) 339-5142

4. Licensed Thermal Power (MUt):

5. Nameplate Rating (Gross MWe):

6. Design Electrical Rating (Net MWe):

7. Maximum Dependable Capacity (Gross WWe):

8. Maximum Dependable Capacity (Net MWe):

9. If Changes Occurred Above Since Last Report, Give Reasons:

10. Power Level To Which Restricted, If Any (Net MHe):

11. Reasons For Restrictions, If Any:

\begin{tabular}{|c|c|c|c|}
\hline & MONTH & YEAR & CUPALATIVE \\
\hline 12. Report Period Hrs & 744.0 & $8,760.0$ & $153,457.0$ \\
\hline 13. Hours Reactor Critical & 744.0 & $6,587.7$ & $101,719.8$ \\
\hline 14. Rx Reserve Shtdwn Hrs & 0.0 & 0.0 & 0.0 \\
\hline 15. Hrs Generator On-Line & 744.0 & $5,868.4$ & $97,756.4$ \\
\hline 16. Unit Reserve Shtdwn Hrs & 0.0 & 0.0 & 0.0 \\
\hline 17. Gross Therm Ener (MUH) & $2,357,760.0$ & $19.279,588.8$ & $310,051,181.8$ \\
\hline 18. Gross Elec Ener (MUH) & $797,350.0$ & $6,075,180.0$ & $102,647,150.0$ \\
\hline 19. Net Elec Ener (MWH) & $764,414.0$ & $5,744,611.0$ & $97,682,164.0$ \\
\hline 20. Unit Service Factor & 100.0 & 67.0 & 63.7 \\
\hline 21. Unit Avail Factor & 100.0 & 67.0 & 63.7 \\
\hline 22. Unit Cap Factor (MDC Net) & 92.9 & 59.3 & 57.6 \\
\hline 23. Unit Cap Factor (DER Net) & 92.1 & 58.8 & 57.1 \\
\hline 24. Unit Forced Outage Rate & 0.0 & 25.9 & 21.3 \\
\hline 25. Forced Outage Hours & 0.0 & 2051.8 & $26,495.9$ \\
\hline
\end{tabular}

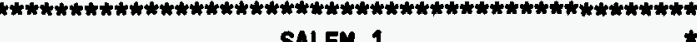
* SALEM 1

AVERAGE DAILY POWER LEVEL (Net MWe)

\begin{tabular}{lr} 
DAY & PONER \\
\hline 1 & 847 \\
\hline 2 & 994 \\
\hline 3 & 1111 \\
\hline 4 & 1120 \\
\hline 5 & 1106 \\
\hline 6 & 1120 \\
\hline 7 & 1079 \\
\hline 8 & 938 \\
\hline 9 & 902 \\
\hline 10 & 1026 \\
\hline 11 & 824 \\
\hline 12 & 552 \\
\hline 13 & 520 \\
\hline 14 & 744 \\
\hline 15 & 1098 \\
\hline
\end{tabular}

\begin{tabular}{ll} 
DAY & PONER \\
\hline 16 & 1122 \\
\hline 17 & 1106 \\
\hline 18 & 1120 \\
\hline 19 & 1124 \\
\hline 20 & 1118 \\
\hline 21 & 1116 \\
\hline 22 & 1134 \\
\hline 23 & 1102 \\
\hline 24 & 1086 \\
\hline 25 & 1126 \\
\hline 26 & 1123 \\
\hline 27 & 1123 \\
\hline 28 & 1124 \\
\hline 29 & 1103 \\
\hline 30 & 1117 \\
\hline 31 & 1117 \\
\hline
\end{tabular}

26. Shutdowns Scheduled Over Mext Six Months (Type, Date, Duration): REFUELING OUTAGE, APRIL 8, 1995, 60 DAYS.

27. If Currently Shutdown, Est imated Startup Date:

Notes:

LICENSEE REVISED MAY 1994 GROSS THERMAL ENERGY FROM 0.0 TO $2,376.0$. 


\begin{tabular}{cccccccccc}
\hline No. & Date & Type & Hours & Reason & Method & LER Number & System & Component & Cause and Corrective ACtion To P \\
\hline 2001 & $12 / 01 / 94$ & F & 0.0 & A & 5 & HF & FILTER & CIRC. MATER TRASH RAKE. \\
2105 & $12 / 07 / 94$ & F & 0.0 & A & 5 & HF & FILTTR & CIRCULATING WATER TRASH RAKE. \\
2022 & $12 / 09 / 94$ & F & 0.0 & A & 5 & IB & IMSTRU & 1A \& 1B S.E.C. IMOPERABLE. \\
2028 & $12 / 11 / 94$ & F & 0.0 & A & 5 & HF & PUMPXX & CIRCULATING MATER PUMPS. \\
2058 & $12 / 14 / 94$ & F & 0.0 & A & 5 & HF & PUMPXX & CIRCULATING WATER PUMPS.
\end{tabular}

\begin{tabular}{|c|c|}
\hline TYPE & REASON \\
\hline $\begin{array}{l}\text { F: Forced } \\
\text { S: Scheduled }\end{array}$ & $\begin{array}{l}\text { A-Equipment Failure } \\
\text { B-Maintenance or Test } \\
\text { C-Refuel ing } \\
\text { D-Regulatory Restriction } \\
\text { E-Operator Training \& } \\
\text { License Examination } \\
\text { F-Adninistrative } \\
\text { G-Operational Error } \\
\text { H-Other }\end{array}$ \\
\hline
\end{tabular}

\begin{tabular}{l} 
METHOD \\
\hline 1-Manual \\
2-Manual Scram \\
3-Auto Scram \\
4-Cont inued \\
5-Reduced Load \\
9-Other
\end{tabular}

\begin{tabular}{ll} 
SYSTEM & COMPONENT \\
\hline $\begin{array}{l}\text { IEEE Standard } \\
805-1984 \text { and/or } \\
\text { NUREG-0161 Exhibit F }\end{array}$ & $\begin{array}{l}\text { IEEE Standard } \\
\text { MUAA-1983 and/or } \\
\text { MUREG-0161 Exhibit H }\end{array}$
\end{tabular}

G-Operational Error 


\section{FACILITY DESCRIPTION}

\section{LOCATION}

STATE.

COUNTY.

NEW JERSEY

SALEM

DIST AND DIRECTION FROM

NEAREST POPULATION CTR...

TYPE OF REACTOR.

DATE INITIAL CRITICALITY...... DECEMBER 11, 1976

DATE INITIAL ELECTRICITY...... DECEMBER 25, 1976

DATE COMMERCIAL OPERATE....... JUNE 30, 1977

CONDENSER COOLING METHOD...... ONCE THRU

CONDEMSER COOLIMG WATER....... DELAMARE RIVER

ELECTRIC RELIABILITY

COUNCIL................... MID-ATLANTIC AREA COUNCIL

\section{UTILITY \& CONTRACTOR INFORMATION}

UTILITY

LICENSEE.

CORPORATE ADDRESS

\section{CONTRACTOR}

ARCHITECT/ENGINEER.

NUC STEAM SYS SUPPLIER...... MESTINGHOUSE

CONSTRUCTOR.

TURBINE SUPPLIER.

UNITED ENG. \& CONSTRUCTORS

WEST INGHOUSE

\section{REGULATORY INFORMATION}

IE REGION RESPONSIBLE.

IE RESIDENT INSPECTOR

CHARLES MARSCHALL

LICENSING PROJ MANAGER

JAMES C. STONE

DOCKET NUMBER

$50-272$

LICENSE \& DATE ISSUAMCE. 


\section{Docket: $50-311$}

OPERATING STATUS

2. Reporting Period: DECEMBER 1994

Outage + On-Line Hrs: $\quad 744.0$

3. Utility Contact: MIKE MORRONI (609) 339-5142

4. Licensed Thermal Power (MWt):

5. Nameplate Rating (Gross MUe):

6. Design Electrical Rating (Net MUe):

7. Maximm Dependable Capacity (Gross MHe):

8. Maximum Dependable Capacity (Net MHe):

9. If Changes Occurred Above Since Last Report, Give Reasons:

10. Power Level To Which Restricted, If Any (Net MWe):

11. Reasons for Restrictions, If Any:

\begin{tabular}{|c|c|c|c|}
\hline & MONTH & YEAR & CUMULATIVE \\
\hline 12. Report Period Hrs & 744.0 & $8,760.0$ & $115,873.0$ \\
\hline 13. Hours Reactor Critical & 0.0 & $6,335.6$ & $75,615.1$ \\
\hline 14. Rx Reserve Shtdun Hrs & 0.0 & 0.0 & 0.0 \\
\hline 15. Hrs Generator On-Line & 0.0 & $6,078.4$ & $72,967.9$ \\
\hline 16. Unit Reserve Shtdwn Hrs & 0.0 & 0.0 & 0.0 \\
\hline 17. Gross Therm Ener (MWH) & 0.0 & $18,510,844.8$ & $180,973,784.2$ \\
\hline 18. Gross Elec Ener (MWH) & 0.0 & $5,903,490.0$ & $76,449,969.0$ \\
\hline 19. Net Elec Ener (MHH) & $(8,818.0)$ & $5,596,267.0$ & $72,723,545.0$ \\
\hline 20. Unit Service Factor & 0.0 & 69.4 & 63.0 \\
\hline 21. Unit Avail Factor & 0.0 & 69.4 & 63.0 \\
\hline 22. Unit Cap Factor (MDC Net) & 0.0 & 57.8 & 56.7 \\
\hline 23. Unit Cap Factor (DER Net) & 0.0 & 57.3 & 56.3 \\
\hline 24. Unit Forced Outage Rate & 0.0 & 8.7 & 21.7 \\
\hline 25. Forced Outage Hours & 0.0 & 575.8 & $20,178.0$ \\
\hline
\end{tabular}

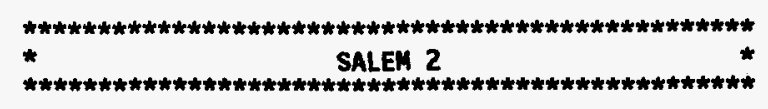

AVERAge DAILY PONER LEVEL (Net MHe)

\begin{tabular}{|c|c|c|c|}
\hline DAY & POUER & DAY & POWER \\
\hline 1 & 0 & 16 & 0 \\
\hline 2 & 0 & 17 & 0 \\
\hline 3 & 0 & 18 & 0 \\
\hline 4 & 0 & 19 & 0 \\
\hline 5 & 0 & 20 & 0 \\
\hline 6 & 0 & 21 & 0 \\
\hline 7 & 0 & 22 & 0 \\
\hline 8 & 0 & 23 & 0 \\
\hline 9 & 0 & 24 & 0 \\
\hline 10 & 0 & 25 & 0 \\
\hline 11 & 0 & 26 & 0 \\
\hline 12 & 0 & 27 & 0 \\
\hline 13 & 0 & 28 & 0 \\
\hline 14 & 0 & 29 & 0 \\
\hline 15 & 0 & 30 & 0 \\
\hline & & 31 & 0 \\
\hline
\end{tabular}

26. Shutdowns Scheduled Over Next Six Months (Type, Date, Duration):

27. If Currently Shutdown, Estimated Startup Date: 01/13/95

Notes: 
TYPE

\begin{tabular}{l} 
REASON \\
\hline A-Equipment Failure \\
B-Maintenance or Test \\
C-Refuel ing \\
D-Regulatory Restriction \\
E-Operator Training \& \\
License Examination \\
F-Administrat ive \\
G-Operational Error \\
H-Other
\end{tabular}

METHOD 1-Manual 2-Manual Scram 3-Auto Scram 4-Cont inued 5-Reduced Load 9-0ther

\section{COMPONENT}

\section{SYSTEM \\ IEEE Standard}

05-1984 and/or

NUREG-0161 Exhibit $F$

\section{IEEE Standard}

803A-1983 and/o

NUREG-0161 Exhibit H

F-Administrativ

H-Other 


\section{FACILITY DESCRIPTION}

\section{LOCATION}

STATE................. NEW JERSEY
COUNTY................ SALEM

DIST AND DIRECTION FROM

NEAREST POPULATION CTR..... 18 MI S OF HILMINGTON, DE

TYPE OF REACTOR............ PHR

DATE INITIAL CRITICALITY...... AUGUST 08, 1980

DATE IAITIAL ELECTRICITY..... JUNE 03, 1981

DATE COMmERCIAL OPERATE....... OCTOBER 13, 1981

CONDENSER COOLING METHOD...... ONCE THRU

CONDENSER COOLING WATER....... DELAMARE RIVER

ELECTRIC RELIABILITY

COUNCIL................... MID-ATLANTIC AREA COUNCIL

\section{UTILITY \& CONTRACTOR INFORMATION}

UTILITY

LICENSEE................. PUbLIC SERVICE ELECTRIC \& GAS Co.

CORPORATE ADDRESS.......... 80 PARK PLACE

NEWARK, MEU JERSEY 07101

\section{CONTRACTOR}

ARCHITECT/ENGIMEER......... PUBLIC SERVICES AND GAS COMPANY

NUC STEAM SYS SUPPLIER...... . WESTINGHOUSE

CONSTRUCTOR

UNITED ENG. \& CONSTRUCTORS

TURBINE SUPPLIER.

WESTINGHOUSE

\section{REGULATORY INFORMATION}

IE REGION RESPONSIBLE.......... 1

IE RESIDENT INSPECTOR......... CHARLES MARSCHALL

LICENSING PROJ MANAGER......... JAMES C. STONE

DOCKET NUMBER............., 50-311

LICENSE \& DATE ISSUANCE......... DPR 075, MAY 20, 1981 
1. Docket: $50-361$

OPERATING STATUS

2. Reporting Period: DECEMBER 1994

Outage + On-Line Hrs: $\mathbf{7 4 4 . 0}$

3. Utility Contact: J. L. DARLING (714) 368-6223

4. Licensed Thermal Power (mWt):

5. Nameplate Rating (Gross MWe):

6. Design Electrical Rating (Net MWe):

7. Maximum Dependable Capacity (Gross MUe):

8. Maximum Dependable Capacity (Net MWe):

9. If Changes Occurred Above Since Last Report, Give Reasons:

10. Power Level To thich Restricted, If Any (Net MWe):

11. Reasons For Restrictions, If Any:

\begin{tabular}{|c|c|c|c|}
\hline Report Period Hrs & $\begin{array}{r}\text { MONTH } \\
744.0\end{array}$ & $\begin{array}{l}\text { YEAR } \\
8,760.0 \\
\end{array}$ & $\begin{array}{r}\text { CUMULATIVE } \\
99,697.0 \\
\end{array}$ \\
\hline Hours Reactor Critical & 744.0 & $8,760.0$ & $76,774.5$ \\
\hline Rx Reserve Shtdwn Hrs & 0.0 & 0.0 & 0.0 \\
\hline Hrs Generator on-Line & 744.0 & $8,760.0$ & $75,631.3$ \\
\hline Unit Reserve Shtdwn Hrs & 0.0 & 0.0 & 0.0 \\
\hline Gross Therm Ener (MWH) & $2,522,349.1$ & $28,803,599.4$ & $247,377,855.8$ \\
\hline Gross Elec Ener (MUH) & $835,070.0$ & $9,763,711.5$ & $83,851,500.5$ \\
\hline Net Elec Ener (MMH) & $797,737.0$ & $9,309,396.0$ & $79,564,094.8$ \\
\hline Unit Service factor & 100.0 & 100.0 & 75.9 \\
\hline Unit Avail Factor & 100.0 & 100.0 & 75.9 \\
\hline Unit Cap Factor (MDC Net) & 100.2 & 99.3 & 74.6 \\
\hline Unit Cap Factor (DER Met) & 100.2 & 99.3 & 74.6 \\
\hline Unit Forced Outage Rate & 0.0 & 0.0 & 5.5 \\
\hline Forced Outage Hours & 0.0 & 0.0 & $4,403.1$ \\
\hline
\end{tabular}

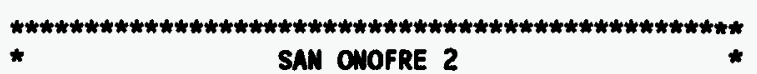

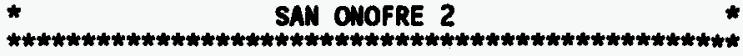

aVerage daily pOWer level (Net mue)

\begin{tabular}{ll} 
DAY & PONER \\
\hline 1 & 1078 \\
\hline 2 & 1076 \\
\hline 3 & 1076 \\
\hline 4 & 1075 \\
\hline 5 & 1076 \\
\hline 6 & 1075 \\
\hline 7 & 1076 \\
\hline 8 & 1076 \\
\hline 9 & 1054 \\
\hline 10 & 982 \\
\hline 11 & 1078 \\
\hline 12 & 1078 \\
\hline 13 & 1079 \\
\hline 14 & 1071 \\
\hline 15 & 1077 \\
\hline
\end{tabular}

\begin{tabular}{ll} 
DAY & PONER \\
\hline 16 & 1078 \\
\hline 17 & 1069 \\
\hline 18 & 1078 \\
\hline 19 & 4078 \\
\hline 20 & 1078 \\
\hline 21 & 1077 \\
\hline 22 & 1077 \\
\hline 23 & 1076 \\
\hline 24 & 1075 \\
\hline 25 & 1075 \\
\hline 26 & 1076 \\
\hline 27 & 1076 \\
\hline 28 & 1075 \\
\hline 29 & 1075 \\
\hline 30 & 1075 \\
\hline 31 & 1074 \\
\hline
\end{tabular}

26. Shutdowns Scheduled Over Next Six Months (Type, Date, Duration): REFUeling OUtAGE, FebruarY 11, 1995, 64 DAYS.

27. If Currently Shutdown, Est imated Startup Date: Notes: 
TYPE

F: Forced

S: Scheduled

\begin{tabular}{l} 
REASON \\
\hline A-Equipment Failure \\
B-Maintenance or Test \\
C-Refuel ing \\
D-Regulatory Restriction \\
E-Operator Training \& \\
License Examination \\
F-Administrative \\
G-Operational Error \\
H-Other
\end{tabular}

METHOD

1-Manua

2-Manual Screm

3-Auto Scram

4-Cont inued

5-Reduced Load

9-0ther
SYSTEM

IEEE Standard

805-1984 and/or

NUREG-0161 Exhibit F
COMPONENT

IEEE Standard

803A-1983 and/or

NUREG-0161 Exhibit H
G-Operational Error

Page 2-254 


\section{UTILITY \& CONTRACTOR INFORMATION}

UTILITY

LICENSEE.

CORPORATE ADDRESS.

SAN DIEGO

\section{DIST AND DIRECTION FROM}

MEAREST POPULATION CTR..... 5 MI SE OF SAN CLEMENTE, CA

TYPE OF REACTOR.

PWR

DATE INITIAL CRITICALITY...... JULY 26, 1982

DATE INITIAL ELECTRICITY...... SEPTEMBER 20, 1982

DATE COMMERCIAL OPERATE....... AUGUST 08, 1983

CONDENSER COOLING METHOD...... ONCE THRU

CONDENSER COOLING WATER....... PACIFIC OCEAN

ELECTRIC RELIABILITY

COUNCIL................... WESTERN SYSTEMS COORDINATION COUNCIL

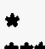
SAN ONOFRE 2

\section{CONTRACTOR}

ARCHITECT/ENGINEER. .

NUC STEAM SYS SUPPLIER

CONSTRUCTOR

TURBINE SUPPLIER

\section{REGULATORY INFORMATION}

IE REGION RESPONSIBLE.........

IE RESIDENT INSPECTOR......... JAMES SLOAN

LICENSING PROJ MANAGER......... MEL B. FIELDS

DOCKET NUMBER.............. 50-361

LICENSE \& DATE ISSUANCE........ MPF 010, SEPTEMBER 07, 1982 


\section{Docket: $50-362$}

OPERATING

$S$ T A TUS

2. Reporting Period: OECEMBER 1994 Outage + On-Line Hrs: 744.0

3. Utility Contact: J. L. DARLING (714) 368-6223

4. Licensed Thermal Power (MUt):

5. Nameplate Rating (Gross Mue):

6. Design Electrical Rating (Net MWe):

7. Maximu Dependable Capacity (Gross MHe):

8. Maximum Dependable Capacity (Net MHe):

9. If Changes Occurred Above Since Last Report, Give Reasons:

10. Power Level To thich Restricted, If Any (Net MHe):

11. Reasons For Restrictions, If Any:

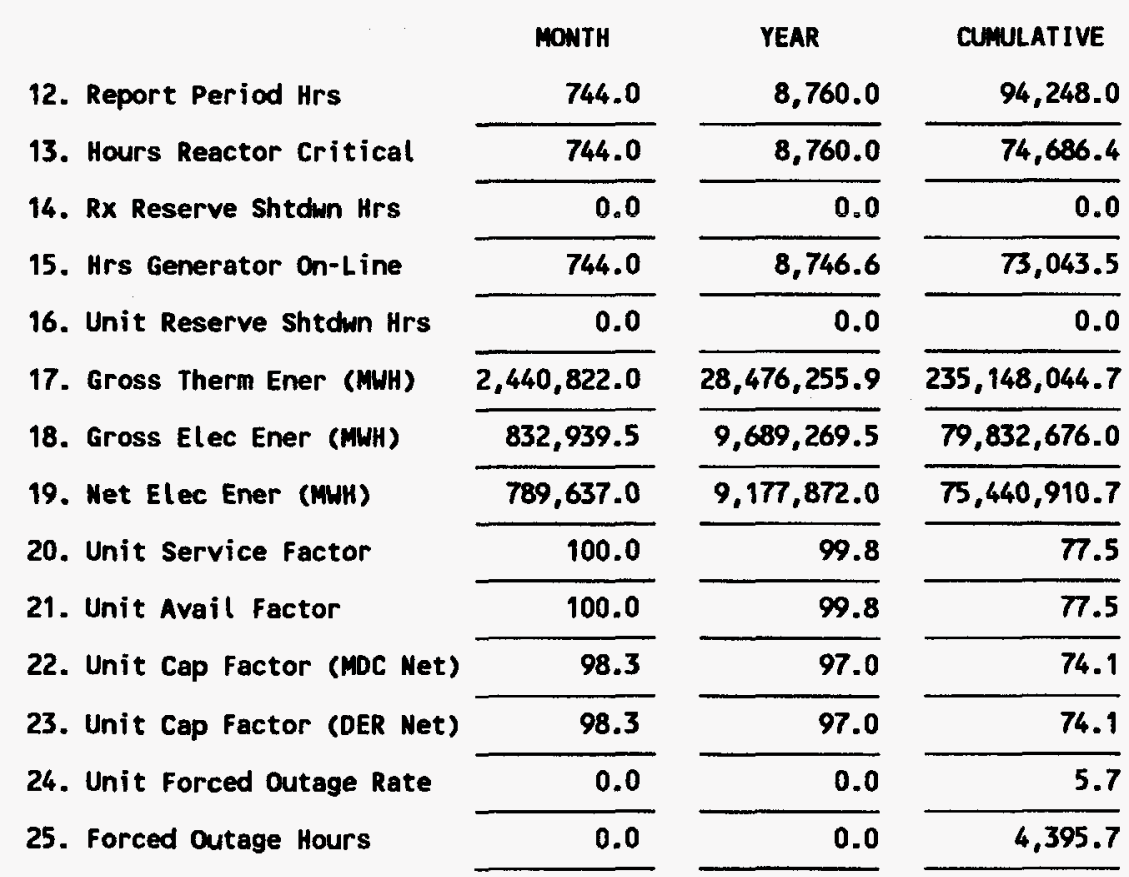

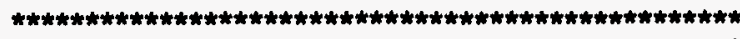

* SAN OMOFRE 3 *

AVERAGe dAILY PONER LeVel (Net Me)

\begin{tabular}{ll} 
DAY & PONER \\
\hline 1 & 1064 \\
\hline 2 & 1063 \\
\hline 3 & 1061 \\
\hline 4 & 1062 \\
\hline 5 & 1062 \\
\hline 6 & 1062 \\
\hline 7 & 1058 \\
\hline 8 & 1062 \\
\hline 9 & 1062 \\
\hline 10 & 1061 \\
\hline 11 & 1062 \\
\hline 12 & 1061 \\
\hline 13 & 1061 \\
\hline 14 & 1062 \\
\hline 15 & 1063 \\
\hline
\end{tabular}

\begin{tabular}{cc} 
DAY & POWER \\
\hline 16 & 1063 \\
\hline 17 & 1062 \\
\hline 18 & 1062 \\
\hline 19 & 1061 \\
\hline 20 & 1062 \\
\hline 21 & 1061 \\
\hline 22 & 1062 \\
\hline 23 & 1061 \\
\hline 24 & 1050 \\
\hline 25 & 1062 \\
\hline 26 & 1061 \\
\hline 27 & 1060 \\
\hline 28 & 1060 \\
\hline 29 & 1061 \\
\hline 30 & 1063 \\
\hline 31 & 1064 \\
\hline
\end{tabular}

26. Shutdowns Scheduled Over Next Six Months (Type, Date, Duration):

27. If Currently Shutdown, Estimated Startup Date:

Notes:

LICENSEE REVISED APRIL 1994 GENERATOR ON LIME HOURS FROM 701.1 TO 705.6 . 
TYPE

F: Forced

S: Scheduled
REASON

A-Equipment Failure B-Maintenance or Test

C-Refueling

D-Regulatory Restriction

E-Operator Training

License Examination

F-Adminis trative

G-Operational Error

H-Other

\section{SYSTEM}

IEEE Standard

805-1984 and/or

NUREG-0161 Exhibit $F$
COMPONENT

IEEE Standard

803A-1983 and/or

NUREG-0161 Exhibit H
2-Manual Scran

3-Auto Scram

5-Reduced Load

9-0ther 


\section{FACILITY DESCRIPTION}

\section{LOCATION}

COUNTY.

ALI FORNIA

SAN DIEGO

\section{DIST AND DIRECTION FROM}

NEAREST POPULATION CTR..... 5 MI SE OF SAN CLEMENTE, CA

TYPE OF REACTOR............. PUR

DATE INITIAL CRITICALITY...... AUGUST 29, 1983

DATE IHITIAL ELECTRICITY...... SEPTEMBER 25, 1983

DATE COMHERCIAL OPERATE....... APRIL 01, 1984

CONDEMSER COOLING METHOD...... ONCE THRU

CONDENSER COOLING MATER....... PACIFIC OCEAN

ELECTRIC RELIABILITY

COUNCIL.................. WESTERH SYSTEMS COORDIMATION

\section{UTILITY \& CONTRACTOR INFORMATION}

\section{UTILITY}

LICENSEE.

CORPORATE ADDRESS.

SOUTHERN CALIFORNIA EDISON CO.

P.0. BOX 800

ROSEMEAD, CALIFORNIA 91770

CONTRACTOR

ARCHITECT/ENGINEER......... BECHTEL

MUC STEAM SYS SUPPLIER...... COMBUSTION ENGINEERING

CONSTRUCTOR............. BECHTEL

TURBINE SUPPLIER........... GEMERAL ELECTRIC CON (ENG VERSION)

\section{REGULATORY INFORMATION}

IE REGION RESPONSIBLE........ 4

IE RESIDENT INSPECTOR

JAMES SLOAN

LICENSING PROJ MANAGER

MEL B. FIELDS

DOCKET NUMBER .

$50-362$

LICENSE \& DATE ISSUANCE 
1. Docket: $50-443$

2. Reporting Period: DECEMBER 1994

OPERATING STATUS

3. Utility Contact: P. E. NARDONE (603) 474-9521 EXT. 4074

4. Licensed Thermal Power (MWt):

5. Nameplate Rating (Gross MHe):

6. Design Electrical Rating (Net MHe):

7. Maximum Dependable Capacity (Gross MWe):

8. Maximum Dependable Capacity (Net MWe):

9. If Changes Occurred Above Since Last Report, Give Reasons:

10. Power Level To Which Restricted, If Any (Net MWe):

11. Reasons For Restrictions, If Any:

\begin{tabular}{|c|c|c|c|}
\hline & MONTH & YEAR & CUMULATIVE \\
\hline 12. Report Periad Hrs & 744.0 & $8,760.0$ & $71,953.0$ \\
\hline 13. Hours Reactor Critical & 744.0 & $5,559.9$ & $33,267.0$ \\
\hline 14. RX Reserve Shtdun Hrs & 0.0 & 0.0 & 953.3 \\
\hline 15. Hrs Generator On-Line & 744.0 & $5,467.0$ & $31,144.2$ \\
\hline 16. Unit Reserve Shtdun Hrs & 0.0 & 0.0 & 0.0 \\
\hline 17. Gross Therm Ener (MUH) & $2,537,531.0$ & $18,404,361.0$ & $101,836,809.0$ \\
\hline 18. Gross Elec Ener (MUH) & $891,902.0$ & $6,450,590.0$ & $35,424,335.0$ \\
\hline 19. Net Elec Ener (MWH) & $858,246.0$ & $6,203,498.0$ & $34,027,117.0$ \\
\hline 20. Unit Service Factor & 100.0 & 62.4 & 77.8 \\
\hline 21. Unit Avail Factor & 100.0 & 62.4 & 77.8 \\
\hline 22. Unit Cap Factor (MDC Net) & 100.3 & 61.6 & 74.9 \\
\hline 23. Unit Cap Factor (DER Net) & 100.5 & 61.7 & 75.1 \\
\hline 24. Unit Forced Outage Rate & 0.0 & 9.2 & 6.8 \\
\hline 25. Forced Outage Hours & 0.0 & 553.3 & $2,532.2$ \\
\hline
\end{tabular}

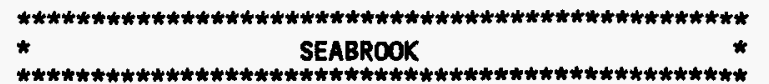

AVERAGE DAILY PONER LEVEL (Net MHe)

\begin{tabular}{ll} 
DAY & PONER \\
\hline 1 & 1154 \\
\hline 2 & 1154 \\
\hline 3 & 1154 \\
\hline 4 & 1154 \\
\hline 5 & 1154 \\
\hline 6 & 1155 \\
\hline 7 & 1154 \\
\hline 8 & 1154 \\
\hline 9 & 1154 \\
\hline 10 & 1154 \\
\hline 11 & 1154 \\
\hline 12 & 1153 \\
\hline 13 & 1153 \\
\hline 14 & 1153 \\
\hline 15 & 1153 \\
\hline
\end{tabular}

\begin{tabular}{cc} 
DAY & POWER \\
\hline 16 & 1153 \\
\hline 17 & 1154 \\
\hline 18 & 1154 \\
\hline 19 & 1154 \\
\hline 20 & 1153 \\
\hline 21 & 1154 \\
\hline 22 & 1154 \\
\hline 23 & 1153 \\
\hline 24 & 1154 \\
\hline 25 & 1154 \\
\hline 26 & 1153 \\
\hline 27 & 1153 \\
\hline 28 & 1153 \\
\hline 29 & 1153 \\
\hline 30 & 1153 \\
\hline 31 & 1153 \\
\hline
\end{tabular}

26. Shutdowns Scheduled Over Mext Six Months (Type, Date, Duration):

\section{If Currently Shutdown, Estimated Startup Date:}

Notes:

CUMULATIVE VALUES FOR ITEMS 12-19 INCLUDE PRE-COMMERCIAL DATA, CUMULATIVE VALUES FOR ITEMS $12-19$ INCLUDE PRE-COMMERCIAL DATA,
UHILE CUMULATIVE VALUES FOR ITEMS 20-25 ARE CALCULATED SINCE COMMERCIAL OPERATION. 


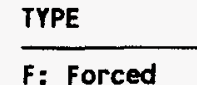

F: Forced

S: Scheduled

\begin{tabular}{l} 
REASON \\
\hline A-Equipment Failure \\
B-Maintenance or Test \\
C-Refuel ing \\
D-Regulatory Restriction \\
E-Operator Training \& \\
License Examination \\
F-Adninistrative \\
G-Operational Error \\
H-Other
\end{tabular}

G-Operational Error

H-other

\section{SYSTEM}

1-Manual

1-Manual

2-Manual scram

3-Auto Seram

4-Cont inued

5-Reduced Load

9-0ther

\section{COMPONENT}

IEEE Standard

\section{IEEE Standard}

NUREG-0161 Exhibit F

NUREG-0161 Exhibit 


\section{FACILITY DESCRIPTION}

\section{LOCATION}

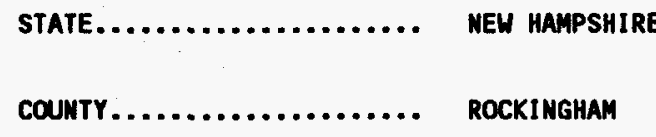

\section{DIST AND DIRECTION FRON}

NEAREST POPULATION CTR..... 13 MI S OF PORTSMOUTH, NH

TYPE OF REACTOR

DATE INITIAL CRITICALITY

DATE INITIAL ELECTRICITY.

DATE COMAERCIAL OPERATE.

CONDENSER COOLING METHOD......

CONDENSER COOLING WATER.

ELECTRIC RELIABILITY

COUNCIL..................... $\begin{aligned} & \text { MORTHEASTERN POWER } \\ & \text { COORDIMATION COUNCIL }\end{aligned}$

\section{UTILITY \& CONTRACTOR INFORMATION}

UTILITY

LICENSEE.

CORPORATE ADDRESS.

NORTH ATLANTIC ENERGY SERVICE CORP.

P.0. BOX 300

SEABROOK, NEW HAMPSHIRE 03874

\section{CONTRACTOR}

ARCHITECT/ENGINEER. .

UNITED ENG. \& CONSTRUCTORS

MUC STEAM SYS SUPPLIER...... MESTINGHOUSE

CONSTRUCTOR

UNITED ENG. \& CONSTRUCTORS

TURBINE SUPPLIER.

GEMERAL ELECTRIC

\section{REGULATORY INFORMATION}

\author{
IE REGION RESPONSIBLE........ 1 \\ IE RESIDENT IMSPECTOR.......... RICHARD LAURA \\ LICENSING PROJ MANAGER......... ALBERT W. DEAGAZIO \\ DOCKET MUMBER.............. 50-443 \\ LICENSE \& DATE ISSUANCE........ NPF 086, MARCH 15, 1990
}


1. Docket: $50-327$

OPERATING STATUS

2. Reporting Period: DECEMBER 1994

Outage + On-Line Hrs: $\quad 744.0$

3. Utility Contact: T. J. HOLLOMON (615) 843-7528

4. Licensed Thermal Power (MUt):

5. Nameplate Rating (Gross MWe):

6. Design Electrical Rating (Net MWe):

7. Maximum Dependable Capacity (Gross MWe):

8. Maximum Dependable Capacity (Net MWe):

9. If Changes Occurred Above Since Last Report, Give Reasons:

10. Power Level To Which Restricted, If Any (Net MWe):

11. Reasons For Restrictions, If Any:

\begin{tabular}{|c|c|c|c|}
\hline & MONTH & YEAR & CUMULATIVE \\
\hline 12. Report Period Hrs & 744.0 & $8,760.0$ & $118,369.0$ \\
\hline 13. Hours Reactor Critical & 632.0 & $6,021.2$ & $62,050.0$ \\
\hline 14. Rx Reserve Shtdwn Hrs & 0.0 & 0.0 & 0.0 \\
\hline 15. Hrs Generator On-Line & 612.9 & 5.775 .8 & $60,604.3$ \\
\hline 16. Unit Reserve Shtdun Hrs & 0.0 & 0.0 & 0.0 \\
\hline 17. Gross Therm Ener (MUH) & $1,967,957.6$ & $18,682,243.4$ & $197,575,997.5$ \\
\hline 18. Gross Elec Ener (MUH) & $680,250.0$ & $6,348,230.0$ & $67,050,884.0$ \\
\hline 19. Net Elec Ener (MUH) & $653,803.0$ & $6,099,376.0$ & $64,263,413.0$ \\
\hline 20. Unit Service Factor & 82.4 & 65.9 & 51.2 \\
\hline 21. Unit Avail Factor & 82.4 & 65.9 & 51.2 \\
\hline 22. Unit Cap Factor (MDC Net) & 79.1 & 62.7 & 48.9 \\
\hline 23. Unit Cap Factor (DER Net) & 76.5 & 60.7 & 47.3 \\
\hline 24. Unit Forced Outage Rate & 17.6 & 6.0 & 36.7 \\
\hline 25. Forced Outage Hours & 131.1 & 365.6 & $35,088.9$ \\
\hline
\end{tabular}

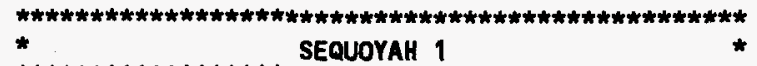

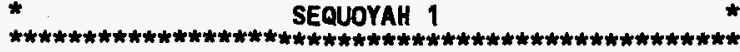

AVERAGE DAILY PONER LEVEL (Net MHe)

\begin{tabular}{ll} 
DAY & PONER \\
\hline 1 & -33 \\
\hline 2 & -30 \\
\hline 3 & -30 \\
\hline 4 & -30 \\
\hline 5 & -33 \\
\hline 6 & -89 \\
\hline 7 & 298 \\
\hline 8 & 653 \\
\hline 9 & 1027 \\
\hline 10 & 1144 \\
\hline 11 & 1144 \\
\hline 12 & 1147 \\
\hline 13 & 1149 \\
\hline 14 & 1150 \\
\hline 15 & 1149 \\
\hline
\end{tabular}

\begin{tabular}{cc} 
DAY & PONER \\
\hline 16 & 1150 \\
\hline 17 & 1151 \\
\hline 18 & 1148 \\
\hline 19 & 1147 \\
\hline 20 & 1147 \\
\hline 21 & 1143 \\
\hline 22 & 1151 \\
\hline 23 & 1152 \\
\hline 24 & 1155 \\
\hline 25 & 1154 \\
\hline 26 & 1156 \\
\hline 27 & 1153 \\
\hline 28 & 1153 \\
\hline 29 & 1155 \\
\hline 30 & 1153 \\
\hline 31 & 1156 \\
\hline
\end{tabular}

26. Shutdowns Scheduled Over Mext Six Months (Type, Date, Duration):

27. If Currently Shutdown, Estimated Startup Date:

Notes: 


\section{Report Period DECEMBER 1994}

$$
\text { UNIT SHUTDOHNS }
$$

AND POHER REDUCTIONS

* SEOUOYAH 1

\begin{tabular}{|c|c|c|c|c|c|c|c|c|c|}
\hline No. & Date & Type & Hours & Reason & Method & LER Number & System & Component & Cause and Corrective Action To Prevent Recurrence \\
\hline 5 & $11 / 29 / 94$ & $\mathbf{F}$ & 131.1 & A & 4 & 94014 & JJ & RJXX & TURBINE AND REACTOR TRIP CONTINUED. \\
\hline
\end{tabular}

TYPE

F: Forced

S: Scheduled

\section{REASON}

A-Equipment Failure A-Equipment Failure
B-Maintenance or Test C-Refuel ing

C-Refueling

D-Regulatory Restriction

-Operator Training \&

License Examination

F-Acministrative

G-Operational

\begin{tabular}{l} 
METHOD \\
\hline 1-Manual \\
2-Manual Scram \\
3-Auto Scram \\
4-Continued \\
5-Reduced Load \\
9-0ther
\end{tabular}

SYSTEM

IEEE Standard

NUREG-0161 Exhibit $F$

5-Reduced Load

IEEE Standard

NUREG-0161 Exhibit H 


\section{FACILITY DESCRIPTION}

\section{LOCATION}

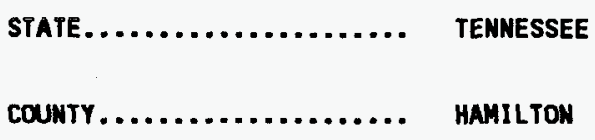

\section{DIST AND DIRECTION FROM}

MEAREST POPULATION CTR..... 9.5 MI NE OF CHATTANOOGA, TN

TYPE OF REACTOR.

PUR

DATE INITIAL CRITICALITY......

DATE INITIAL ELECTRICITY......

DATE COMMERCIAL OPERATE.

CONDENSER COOLING METHOD.

CONDENSER COOLING WATER.

ELECTRIC RELIABILITY

COUNCIL................... SOUTHEASTERN ELECTRIC

\section{UTILITY \& CONTRACTOR INFORMATION}

UTILITY

LICENSEE................ TEMNESSEE VALLEY AUTHORITY

CORPORATE ADDRESS.......... 400 VEST SUMMIT HILL DRIVE KNOXVILLE，TENNESSEE 37933

\section{CONTRACTOR}

ARCHITECT/ENGINEER

TENNESSEE VALLEY AUTHORITY

NUC STEAM SYS SUPPLIER

WESTINGHOUSE

CONSTRUCTOR

TENMESSEE VALLEY AUTHORITY

TURBINE SUPPLIER.

MESTINGHOUSE

\section{REGULATORY INFORMATION}

IE REGION RESPONSIBLE......... 2

IE RESIDENT INSPECTOR......... HILLIAM HOLLAND

LICENSING PROJ MANAGER......... DAVID E. LABARGE

DOCKET MUMBER............. 50-327

LICENSE \& DATE ISSUANCE........ DPR 077, SEPTEMBER 17, 1980 
1. Docket: $50-328$

OPERATING STATUS

2. Reporting Period: DECEMBER 1994

Outage + On-Line Hrs: $\quad 744.0$

3. Utility Contact: T. J. HOLLONON (615) 843-7528

4. Licensed Thermal Power (MWt):

5. Nameplate Rating (Gross MUe):

6. Design Electrical Rating (Net MWe):

7. Maximm Dependable Capacity (Gross MUe):

8. Maximum Dependable Capacity (Net Mue):

9. If Changes Occurred Above Since Last Report, Give Reasons:

10. Power Level To thich Restricted, If Any (Net MWe):

11. Reasons For Restrictions, If Any:

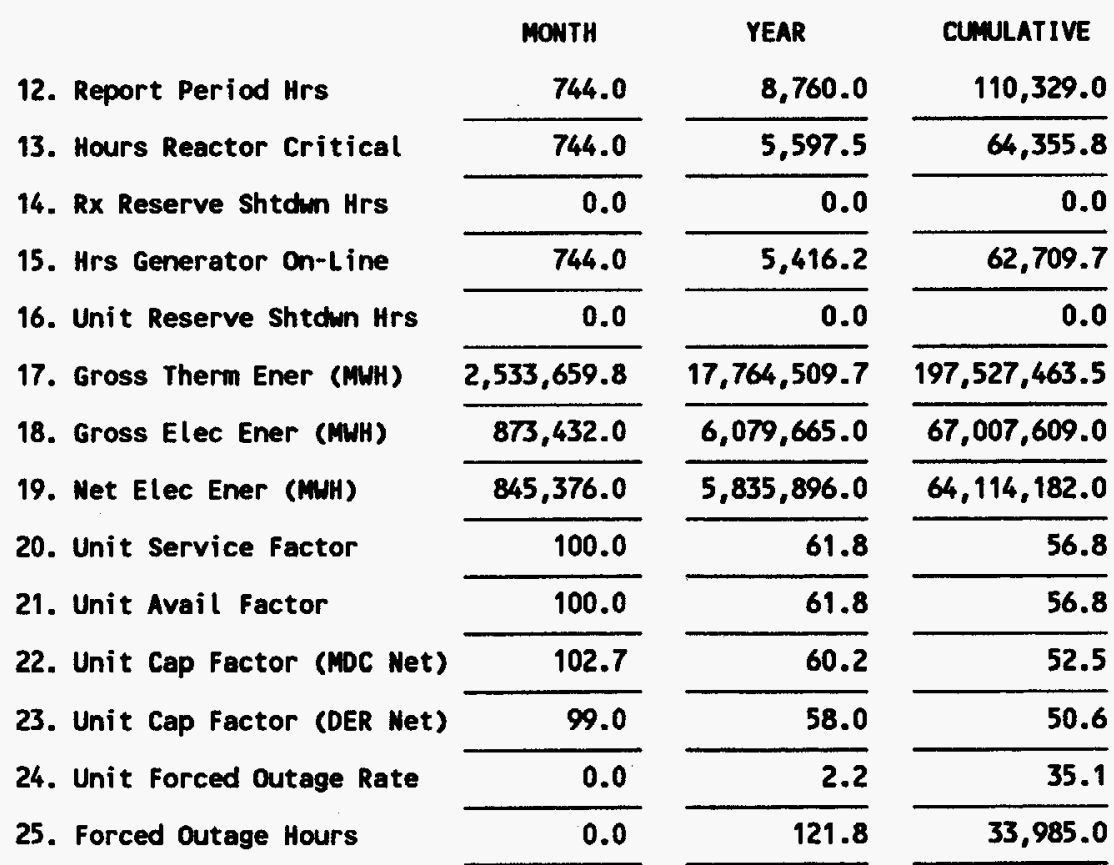

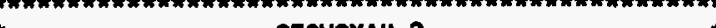 \\ SEQUOYAH $?$}

SEaCOHAH 2

AVERAGE DAILY POWER LEVEL (Net MUE)

\begin{tabular}{ll} 
DAY & PONER \\
\hline 1 & 1151 \\
\hline 2 & 1106 \\
\hline 3 & 1146 \\
\hline 4 & 1118 \\
\hline 5 & 1124 \\
\hline 6 & 1131 \\
\hline 7 & 1132 \\
\hline 8 & 1135 \\
\hline 9 & 1140 \\
\hline 10 & 1138 \\
\hline 11 & 1138 \\
\hline 12 & 1141 \\
\hline 13 & 1143 \\
\hline 14 & 1137 \\
\hline 15 & 1141 \\
\hline
\end{tabular}

\begin{tabular}{ll} 
DAY & PONER \\
\hline 16 & 1145 \\
\hline 17 & 1143 \\
\hline 18 & 1143 \\
\hline 19 & 1131 \\
\hline 20 & 1130 \\
\hline 21 & 1139 \\
\hline 22 & 1133 \\
\hline 23 & 1141 \\
\hline 24 & 1140 \\
\hline 25 & 1143 \\
\hline 26 & 1140 \\
\hline 27 & 1139 \\
\hline 28 & 1140 \\
\hline 29 & 1139 \\
\hline 30 & 1142 \\
\hline 31 & 1143 \\
\hline
\end{tabular}

26. Shutdowns Scheduled Over Next Six Months (Type, Date, Duration):

27. If Currently Shutdown, Estimated Startup Date:

Notes: 
TYPE

F: Forced

F: Forced

\begin{tabular}{l} 
REASON \\
\hline A-Equipment Failure \\
B-Maintenance or Test \\
C-Refuel ing \\
D-Regulatory Restriction \\
E-Operator Training \& \\
License Examination \\
F-Administrative \\
G-operational Error \\
H-Other
\end{tabular}

METHOD

1-Manual

1-Manual

2-Manual Scram

3-Auto Scram

5-Reduced Load

9-other

\section{SYSTEM}

IEEE Standard

MUREG-0161
COMPONENT

IEEE Standard

803A-1983 and/or

NUREG-0161 Exhibit H

F-Administrative

H-other 


\section{FACILITY DESCRIPTION}

\section{LOCATION}

STATE.

COUNTY

TENNESSEE

HAMILTON

\section{DIST AND DIRECTION FROM}

MEAREST POPULATION CTR.

TYPE OF REACTOR.............. PI

DATE INITIAL CRITICALITY...... NOVEMBER 05, 1981

DATE INITIAL ELECTRICITY...... DECEMBER 23, 1981

DATE COMMERCIAL OPERATE....... JUNE 01, 1982

CONDENSER COOLING METHOD...... ONCE THRU

CONDENSER COOLING WATER....... CHICKAMAUGA LAKE

ELECTRIC RELIABILITY

COUNCIL................... SOUTHEASTERN ELECTRIC RELIABILITY COUNCIL

\section{UTILITY \& CONTRACTOR INFORMATION}

\section{UTILITY}

LICENSEE.

TENNESSEE VALLEY AUTHORITY

CORPORATE ADDRESS.

400 MEST SUMAIT HILL DRIVE KNOXVILLE, TEMNESSEE 37933

CONTRACTOR

ARCHITECT/ENGINEER......... TENMESSEE VALLEY AUTHORITY

MUC STEAM SYS SUPPLIER...... WESTIMGHOUSE

CONSTRUCTOR............... TEMHESSEE VALLEY AUTHORITY

TURBINE SUPPLIER........... MESTINGHOUSE

\section{REGULATORY IMFORMATION}

IE REGION RESPONSIBLE........ 2

IE RESIDENT INSPECTOR.

WILLIAM HOLLAND

LICENSING PROJ MANAGER.

DAVIO E. LABARGE

DOCKET MUMBER.

$50-328$

LICENSE \& DATE ISSUANCE. 


\section{Docket: $50-498$}

OPERATING STATUS

2. Reporting Period: DECEMBER 1994

Outage + On-Line Hrs: 744.0

3. Utility contact: R. L. HILL (512) 972-7667

4. Licensed Thermal Power (MWt):

5. Mameplate Rating (Gross MHe):

6. Design Electrical Rating (Net MWe):

7. Maximum Dependable Capacity (Gross MWe):

8. Maximum Dependable Capacity (Net MWe):

9. If Changes Occurred Above Since Last Report, Give Reasons:

10. Power Level To Which Restricted, If Any (Net MWe):

11. Reasons For Restrictions, If Any:

\begin{tabular}{|c|c|c|c|}
\hline & MONTH & YEAR & CUMULATIVE \\
\hline 12. Report Period Hrs & 744.0 & $8,760.0$ & $55,681.0$ \\
\hline 13. Hours Reactor Critical & 744.0 & $7,080.3$ & $33,942.0$ \\
\hline 14. Rx Reserve Shtchn Hrs & 0.0 & 0.0 & 0.0 \\
\hline 15. Hrs Generator On-Line & 744.0 & $6,843.3$ & $32,767.1$ \\
\hline 16. Unit Reserve Shtdwn Hrs & 0.0 & 0.0 & 0.0 \\
\hline 17. Gross Therm Ener (MHH) & $2,833,893.0$ & $25,156,473.0$ & $119,821,979.0$ \\
\hline 18. Gross Elec Ener (MUH) & 979.240 .0 & $8,640,300.0$ & $40,654,960.0$ \\
\hline 19. Net Elec Ener (MUH) & 939.703 .0 & $8,251,408.0$ & $38,510,952.0$ \\
\hline 20. Unit Service Factor & 100.0 & 78.1 & 58.8 \\
\hline 21. Unit Avail Factor & 100.0 & 78.1 & 58.8 \\
\hline 22. Unit Cap Factor (MDC Net) & 101.0 & 75.3 & 55.3 \\
\hline 23. Unit Cap Factor (DER Net) & 101.0 & 75.3 & 55.3 \\
\hline 24. Unit Forced Outage Rate & 0.0 & 21.8 & 30.3 \\
\hline 25. Forced Outage Hours & 0.0 & 1910.8 & $14,226.7$ \\
\hline
\end{tabular}

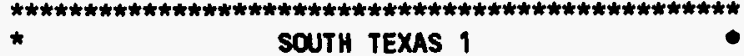

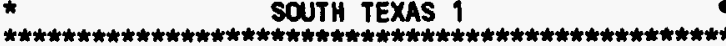

AVERAGE DAILY PONER LEVEL (Net MHe)

\begin{tabular}{ll} 
DAY & PONER \\
\hline & 1268 \\
\hline 2 & 1267 \\
\hline 3 & 1265 \\
\hline 4 & 1267 \\
\hline 5 & 1265 \\
\hline 6 & 1266 \\
\hline 7 & 1263 \\
\hline 8 & 1264 \\
\hline 9 & 1262 \\
\hline 10 & 1268 \\
\hline 11 & 1267 \\
\hline 12 & 1264 \\
\hline 13 & 1265 \\
\hline 14 & 1265 \\
\hline 15 & 1264 \\
\hline
\end{tabular}

\begin{tabular}{|ll} 
DAY & PONER \\
\hline 16 & 1265 \\
\hline 17 & 1220 \\
\hline 18 & 1266 \\
\hline 19 & 1263 \\
\hline 20 & 1267 \\
\hline 21 & 1266 \\
\hline 22 & 1268 \\
\hline 23 & 1268 \\
\hline 24 & 1268 \\
\hline 25 & 1267 \\
\hline 26 & 1266 \\
\hline 27 & 1253 \\
\hline 28 & 1241 \\
\hline 29 & 1264 \\
\hline 30 & 1266 \\
\hline 31 & 1266 \\
\hline
\end{tabular}

26. Shutdowns Scheduled Over Mext six Months (Type, Date, Duration): REFUELING OUTAGE, MARCH 4, 1995, 45 DAYS.

27. If Currently Shutdown, Estimated startup Date:

Notes:

MAXIMUM DEPENDABLE CAPACITY (GROSS MME) NOT PROVIDED. 


\begin{tabular}{l} 
TYPE \\
\hline F: Forced \\
S: Scheduled
\end{tabular}

\begin{tabular}{l} 
REASON \\
\hline A-Equipment Failure \\
B-Maintenance or Test \\
C-Refuel ing \\
D-Regulatory Restriction \\
E-Operator Training \& \\
License Examination \\
F-Administrative \\
G-Operational Error \\
H-Other
\end{tabular}

REASON

METHOD

1-Manual

2-Manual Scram

3-Auto Scram

4-Auto Scram

5-Reduced Load

9-0ther
SYSTEM

IEEE Standard

NUREG-0161 Exhibit F
CONPONENT

IEEE Standard

803A-1983 and/or

NUREG-0161 Exhibit H 


\section{FACILITY DESCRIPTION}

LOCATION

STATE................. TEXAS

COUNTY.

MATAGORDA

DIST AND DIRECTION FROM

MEAREST POPULATION CTR.

TYPE OF REACTOR

PUR

DATE INITIAL CRITICALITY...... MARCH 08, 1988

DATE INITIAL ELECTRICITY...... MARCH 30, 1988

DATE COMERCIAL OPERATE....... AUGUST 25, 1988

CONDENSER COOLING METHOD...... ONCE THRU

CONDENSER COOLIMG WATER....... COLORADO RIVER

ELECTRIC RELIABILITY

COUNCIL................. EAST CENTRAL AREA RELIABILITY COORDINATION AGREEMENT

\section{UTILITY \& CONTRACTOR INFORMATION}

UTILITY

LICENSEE..

HOUSTON LIGHTING \& PONER CO.

CORPORATE ADDRESS

P.0. BOX 1700

HOUSTON, TEXAS 77001

\section{CONTRACTOR}

ARCHITECT/EMGIMEER......... BECHTEL

NUC STEAM SYS SUPPLIER...... MESTINGHOUSE

CONSTRUCTOR.............. EBASCO

TURBIME SUPPLIER.......... WESTINGHOUSE

\section{REGULATORY INFORMATION}

IE REGION RESPONSIBLE........ 4

IE RESIDENT INSPECTOR......... DAVID LOVELESS

LICENSIMG PROJ MAMAGER......... LAURENCE E. KOKAJKO

DOCKET MUMBER.............. $50-498$

LICENSE \& DATE ISSUANCE........ NPF 076, MARCH 22, 1988 
1. Docket: $50-499$

OPERATING STATUS

2. Reporting Period: DECEMBER 1994

Outage + On-Line Hrs: $\mathbf{7 4 4 . 0}$

3. Utility Contact: R. L. HILL (512) $972-7667$

4. Licensed Thermal Power (MWt):

5. Nameplate Rating (Gross MWe):

6. Design Electrical Rating (Net MWe):

7. Maximum Dependable Capacity (Gross MHe):

8. Maximum Dependable Capacity (Net MHe):

9. If Changes Occurred Above Since Last Report, Give Reasons:

10. Power Level To Which Restricted, If Any (Net MHe):

11. Reasons For Restrictions, If Any:

\begin{tabular}{|c|c|c|c|}
\hline & MONTH & YEAR & CUMULATIVE \\
\hline 12. Report Period Hrs & 744.0 & $8,760.0$ & $48,529.0$ \\
\hline 13. Hours Reactor Critical & 744.0 & $5,280.6$ & $30,037.0$ \\
\hline 14. Rx Reserve Shtdwn Hrs & 0.0 & 0.0 & 0.0 \\
\hline 15. Hrs Generator On-Line & 744.0 & $5,099.0$ & $28,832.9$ \\
\hline 16. Unit Reserve Shtdun Hrs & 0.0 & 0.0 & 0.0 \\
\hline 17. Gross Therm Ener (MUH) & $2,719,383.0$ & $18,346,973.0$ & $104,780,221.0$ \\
\hline 18. Gross Elec Ener (МHH) & $937,870.0$ & $6,253,280.0$ & $35,457,870.0$ \\
\hline 19. Net Elec Ener (MUH) & $899,558.0$ & $5,990,976.0$ & $33,726,248.0$ \\
\hline 20. Unit Service Factor & 100.0 & 58.2 & 59.4 \\
\hline 21. Unit Avail Factor & 100.0 & 58.2 & 59.4 \\
\hline 22. Unit Cap Factor (MDC Met) & 96.6 & 54.7 & 55.6 \\
\hline 23. Unit Cap factor (DER Net) & 96.6 & 54.7 & 55.6 \\
\hline 24. Unit Forced Outage Rate & 0.0 & 41.7 & 30.7 \\
\hline 25. Forced Outage Hours & 0.0 & 3652.3 & 12.781 .4 \\
\hline
\end{tabular}

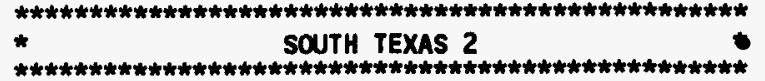

AVERAGE DAILY POWER LEVEL (Net MWe)

\begin{tabular}{ll} 
DAY & PONER \\
\hline 1 & 1269 \\
\hline 2 & 1265 \\
\hline 3 & 1268 \\
\hline 4 & 1094 \\
\hline 5 & 560 \\
\hline 6 & 541 \\
\hline 7 & 1066 \\
\hline 8 & 1265 \\
\hline 9 & 1265 \\
\hline 10 & 1267 \\
\hline 11 & 1268 \\
\hline 12 & 1269 \\
\hline 13 & 1267 \\
\hline 14 & 1265 \\
\hline 15 & 1264 \\
\hline
\end{tabular}

\begin{tabular}{ll} 
DAY & PONER \\
\hline 16 & 1265 \\
\hline 17 & 1266 \\
\hline 18 & 1266 \\
\hline 19 & 1265 \\
\hline 20 & 1266 \\
\hline 21 & 1267 \\
\hline 22 & 1269 \\
\hline 23 & 1269 \\
\hline 24 & 1269 \\
\hline 25 & 1269 \\
\hline 26 & 1269 \\
\hline 27 & 1268 \\
\hline 28 & 1270 \\
\hline 29 & 1269 \\
\hline 30 & 1271 \\
\hline 31 & 1271 \\
\hline
\end{tabular}

26. Shutdowns Scheduled Over Next Six Months (Type, Date, Duration):

27. If Currently Shutdown, Estimated Startup Date:

Notes:

MAXIMUM DEPENDABLE CAPACITY (GROSS MME) NOT PROVIDED. 


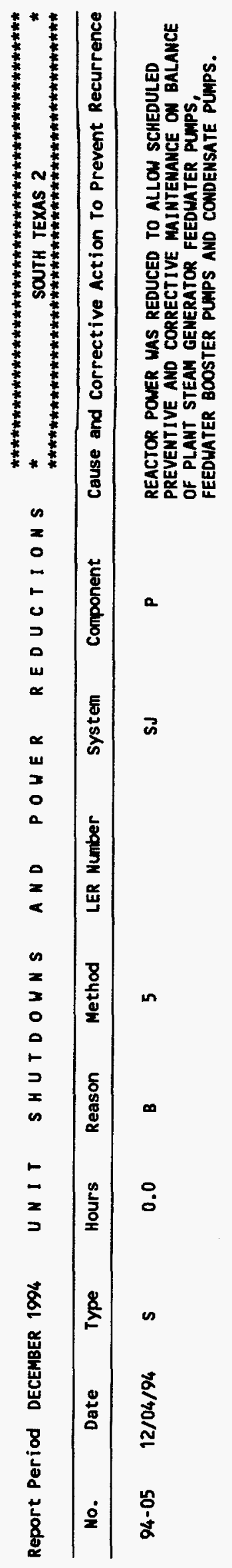

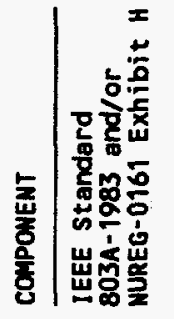

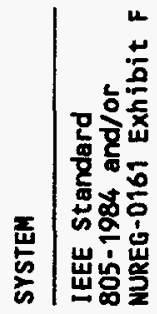

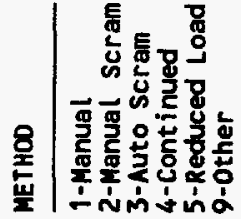

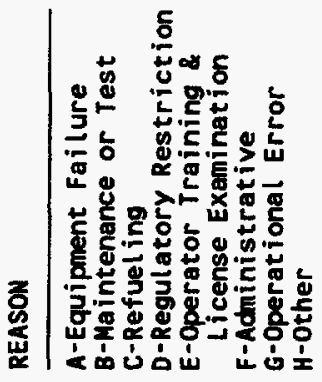

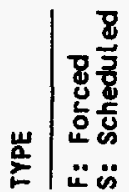




\section{FACILITY DESCRIPTION}

\section{LOCATION}

STATE.

COUNTY

TEXAS

\section{MATAGORDA}

\section{DIST AND DIRECTION FROM} NEAREST POPULATION CTR.....

TYPE OF REACTOR.

DATE INITIAL CRITICALITY.

DATE INITIAL ELECTRICITY......

DATE COMHERCIAL OPERATE.

CONDENSER COOLING METHOD

CONDENSER COOLING MATER

ELECTRIC RELIABILITY

COUNCIL................. EAST CENTRAL AREA RELIABILITY

\section{UTILITY \& CONTRACTOR INFORMATION}

\section{UTILITY}

LICERSEE.................. HOUSTON LIGHTING \& POWER CO.

CORPORATE ADDRESS.......... P.0. BOX 1700

HOUSTON, TEXAS 77001

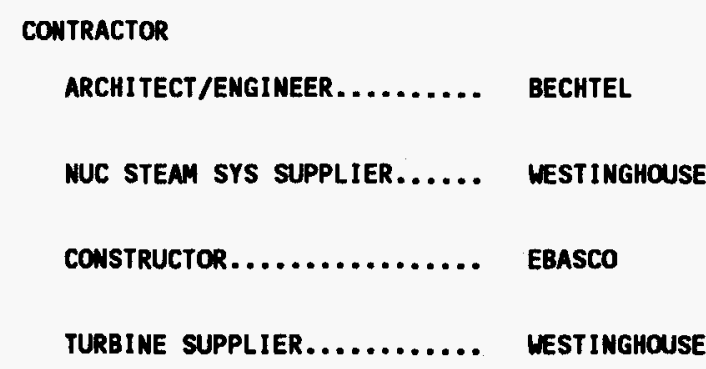

ARCHI TECT/ENGINEER.

BECHTEL

NUC STEAM SYS SUPPLIER.

WEST INGHOUSE

CONSTRUCTOR

EBAsco

TURBINE SUPPLIER

WESTINGHOUSE

\section{REGULATORY INFORMATION}

IE REGION RESPONSIBLE..........

IE RESIDENT INSPECTOR.......... DAVID LOVELESS

LICENSING PROJ MANAGER.

LAMRENCE E. KOKAJKO

DOCKET NUMBER.

$50-499$

LICENSE \& DATE ISSUANCE 


\section{Docket: $50-335$}

OPERATING STATUS

2. Reporting Period: DeCEMBER 1994

Outage + On-Line Hrs:

744.0

3. Utility Contact: GEORGE MADDEN (407) $468-4298$

4. Licensed Thermal Power (NWt):

5. Nameplate Rating (Gross MUe):

6. Design Electrical Rating (Net MWe):

7. Maximum Dependable Capacity (Gross MWe):

8. Maximum Dependable Capacity (Net MHe):

9. If Changes Occurred Above Since Last Report, Give Reasons:

10. Power Level To Which Restricted, If Any (Net MWe):

11. Reasons For Restrictions, If Any:

\begin{tabular}{|c|c|c|c|}
\hline & MONTH & YEAR & CUMULATIVE \\
\hline 12. Report Period Hrs & 744.0 & $8,760.0$ & $158,040.0$ \\
\hline 13. Hours Reactor Critical & 744.0 & $7,793.6$ & $123,323.4$ \\
\hline 14. Rx Reserve Shtdwn Hrs & 0.0 & 0.0 & 205.3 \\
\hline 15. Hrs Generator On-Line & 737.1 & $7,602.0$ & $121,957.0$ \\
\hline 16. Unit Reserve Shtdun Hrs & 0.0 & 0.0 & 39.3 \\
\hline 17. Gross Therm Ener (MNH) & $1,864,030.0$ & $19,968,441.0$ & $315,733,745.0$ \\
\hline 18. Gross Elec Ener (MUH) & $616,450.0$ & $6,536,720.0$ & $104,150,010.0$ \\
\hline 19. Net Elec Ener (MWH) & $583,626.0$ & $6,178,541.0$ & $98,436,903.0$ \\
\hline 20. Unit Service Factor & 99.1 & 86.8 & 77.2 \\
\hline 21. Unit Avail Factor & 99.1 & 86.8 & 77.2 \\
\hline 22. Unit Cap Factor (MDC Net) & 93.5 & 84.1 & 76.5 \\
\hline 23. Unit Cap Factor (DER Net) & 94.5 & 85.0 & 75.9 \\
\hline 24. Unit Forced Outage Rate & 0.0 & 5.1 & 4.0 \\
\hline 25. Forced Outage Hours & 0.0 & 407.0 & $5,116.6$ \\
\hline
\end{tabular}

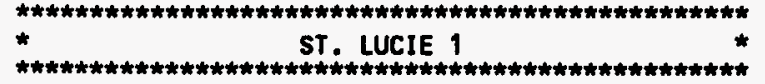

AVERAGE DAILY PONER LEVEL (Net MHe)

\begin{tabular}{lc} 
DAY & PONER \\
\hline 1 & 69 \\
\hline 2 & 203 \\
\hline 3 & 436 \\
\hline 4 & 775 \\
\hline 5 & 814 \\
\hline 6 & 816 \\
\hline 7 & 816 \\
\hline 8 & 816 \\
\hline 9 & 819 \\
\hline 10 & 832 \\
\hline 11 & 849 \\
\hline 12 & 849 \\
\hline 13 & 850 \\
\hline 14 & 851 \\
\hline 15 & 848 \\
\hline
\end{tabular}

\begin{tabular}{cc} 
DAY & PONER \\
\hline 16 & 854 \\
\hline 17 & 852 \\
\hline 18 & 852 \\
\hline 19 & 852 \\
\hline 20 & 854 \\
\hline 21 & 854 \\
\hline 22 & 854 \\
\hline 23 & 854 \\
\hline 24 & 857 \\
\hline 25 & 857 \\
\hline 26 & 856 \\
\hline 27 & 856 \\
\hline 28 & 857 \\
\hline 29 & 857 \\
\hline 30 & 857 \\
\hline 31 & 857 \\
\hline
\end{tabular}

26. Shutdowns Scheduled Over Next Six Months (Type, Date, Duration):

27. If Currently Shutdown, Estimated Startup Date:

Motes:

CUMULATIVE UNIT CAPACITY FACTORS (MDC \& DER) ARE CALCULATED WITH WEIGHTED AVERAGES. 
A N D

REDUCTIONS

* ST. LUCIE 1

\begin{tabular}{|c|c|c|c|c|c|c|c|c|c|}
\hline No. & Date & Type & Hours & Reason & Method & LER Number & System & Component & Cause and Corrective Action To Prevent Recurrence \\
\hline 21 & $10 / 31 / 94$ & $\mathbf{s}$ & 6.9 & c & 4 & & RC & FUELXX & UNIT REMAINED OFF LINE FOR CYCLE REFUELING. \\
\hline 22 & $12 / 01 / 94$ & s & 0.0 & B & 9 & & RC & FUELXX & $\begin{array}{l}\text { PONER HOLD FOR PHYSICS TESTING AND TURBINE } \\
\text { OVERSPEED TRIP TEST. }\end{array}$ \\
\hline 23 & $12 / 02 / 94$ & $\mathbf{F}$ & 0.0 & A & 5 & & RC & FUELXX & $\begin{array}{l}\text { UNIT EXPERIENCING PONER ESCALATION PHYSICS } \\
\text { TESTIMG. }\end{array}$ \\
\hline
\end{tabular}

\begin{tabular}{l} 
TYPE \\
\hline F: Forced \\
S: Scheduled
\end{tabular}

\begin{tabular}{|c|c|}
\hline REASON & METHOO \\
\hline $\begin{array}{l}\text { A-Equipment Fai lure } \\
\text { B-Maintenance or Test } \\
\text { C-Refuel ing } \\
\text { D-Regulatory Restriction } \\
\text { E-Operator Training \& } \\
\text { License Examination } \\
\text { F-Adninistrat ive }\end{array}$ & $\begin{array}{l}\text { 1-Manual } \\
\text { 2-Manual scram } \\
\text { 3-Auto Scram } \\
\text { 4-Cont inued } \\
\text { 5-Reduced Load } \\
\text { 9-0ther }\end{array}$ \\
\hline
\end{tabular}

\section{SYSTEM \\ IEEE Standard}

NUREG-0161 Exhibit

\section{COMPONENT}

IEEE Standard

303A-1983 and/or

NUREG-0161 Exhibit .H

License Examination

(anced

G-Operational Error

H-Other 
FACILITY DESCRIPTION

LOCATION

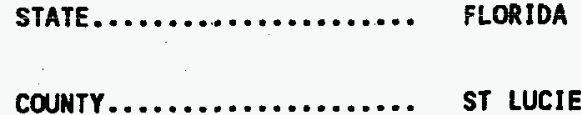

DIST AND DIRECTION FRON

NEAREST POPULATION CTR...... 12 MI SE OF FT. PIERCE, FL

TYPE OF REACTOR

DATE INITIAL CRITICALITY...... APRIL 22, 1976

DATE IMITIAL ELECTRICITY...... MAY 07, 1976

DATE COMMERCIAL OPERATE........ DECEMBER 21, 1976

CONDENSER COOLING METHOD...... ONCE THRU

CONDENSER COOLING WATER....... ATLANTIC OCEAN

ELECTRIC RELIABILITY

COUNCIL................... SOUTHEASTERN ELECTRIC RELIABILITY COUNCIL

\section{UTILITY \& CONTRACTOR IMFORMATION}

UTILITY

LICENSEE.

FLORIDA PONER \& LIGHT CO.

CORPORATE ADDRESS.

\section{WEST FLAGLER STREET}

MIAMI, FLORIDA 33102

\section{CONTRACTOR}

ARCHITECT/ENGIMEER

EBASCO

NUC STEAM SYS SUPPLIER

COMBUSTION ENGINEERING

CONSTRUCTOR

EBASCO

TURBINE SUPPLIER

WESTINGHOUSE

\section{REGULATORY INFORMATION}

IE REGION RESPONSIBLE......... 2

IE RESIDENT INSPECTOR......... RICHARD PREVATTE

LICENSING PROJ MANAGER......... JAN ADAM MORRIS

DOCKET MUMBER.............. 50-335

LICENSE \& DATE ISSUANCE. 
1. Docket: $50-389$

OPERATING STATUS

2. Reporting Period: DECEMBER 1994

Outage + On-Line Hrs: 744.0

3. Utility Contact: GEORGE MADDEN (407) 468-4298

4. Licensed Thermal Power (MUt):

5. Nameplate Rating (Gross MWe):

6. Design Electrical Rating (Net MWe):

7. Maximum Dependable Capacity (Gross MHe):

8. Maximum Dependable Capacity (Net MWe):

9. If Changes Occurred Above Since Last Report, Give Reasons:

10. Power Level To thich Restricted, If Any (Net MUe):

11. Reasons For Restrictions, If Any:

\begin{tabular}{|c|c|c|c|}
\hline & MONTH & YEAR & CUMULATIVE \\
\hline 12. Report Period Hrs & 744.0 & $8,760.0$ & $99,937.0$ \\
\hline 13. Hours Reactor Critical & 744.0 & $7,104.1$ & $84,267.6$ \\
\hline 14. Rx Reserve Shtctun Hrs & 0.0 & 0.0 & 0.0 \\
\hline 15. Hrs Generator on-Line & 744.0 & $6,971.7$ & $82,866.9$ \\
\hline 16. Unit Reserve Shtohn Hrs & 0.0 & 0.0 & 0.0 \\
\hline 17. Gross Therm Ener (MWH) & $1,944,054.0$ & $18,236,614.0$ & $215,177,125.0$ \\
\hline 18. Gross Elec Ener (MHH) & $639,510.0$ & $5,944,160.0$ & $71,453,710.0$ \\
\hline 19. Het Elec Ener (MHH) & $605,392.0$ & $5,604,709.0$ & $67,505,889.0$ \\
\hline 20. Unit Service Factor & 100.0 & 79.6 & 82.9 \\
\hline 21. Unit Avail Factor & 100.0 & 79.6 & 82.9 \\
\hline 22. Unit Cap Factor (MDC Net) & 97.0 & 76.3 & 81.4 \\
\hline 23. Unit Cap Factor (DER Net) & 98.0 & 77.1 & 81.8 \\
\hline 24. Unit Forced Outage Rate & 0.0 & 2.3 & 7.0 \\
\hline 25. Forced Outage Hours & 0.0 & 166.4 & $6,205.8$ \\
\hline
\end{tabular}

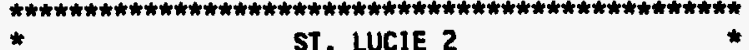

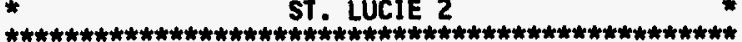

AVERAGE DAILY POWER LEVEL (Net MWe)

\begin{tabular}{lc} 
DAY & PONER \\
\hline 1 & 830 \\
\hline 2 & 834 \\
\hline 3 & 832 \\
\hline 4 & 831 \\
\hline 5 & 829 \\
\hline 6 & 833 \\
\hline 7 & 833 \\
\hline 8 & 833 \\
\hline 9 & 835 \\
\hline 10 & 832 \\
\hline 11 & 796 \\
\hline 12 & 561 \\
\hline 13 & 614 \\
\hline 14 & 647 \\
\hline 15 & 703 \\
\hline
\end{tabular}

\begin{tabular}{|c|c}
\hline DAY & PONER \\
\hline 16 & 808 \\
\hline 17 & 849 \\
\hline 18 & 848 \\
\hline 19 & 850 \\
\hline 20 & 851 \\
\hline 21 & 850 \\
\hline 22 & 851 \\
\hline 23 & 852 \\
\hline 24 & 853 \\
\hline 25 & 853 \\
\hline 26 & 854 \\
\hline 27 & 848 \\
\hline 28 & 854 \\
\hline 29 & 854 \\
\hline 30 & 854 \\
\hline 31 & 853 \\
\hline
\end{tabular}

26. Shutdowns Scheduled Over Next six Months (Type, Date, Duration):

27. If Currently Shutdown, Estimated Startup Date:

Notes:

CUMULATIVE UNIT CAPACITY FACTORS (MDC \& DER) ARE CALCULATED WITH WEIGHTED AVERAGES. 
METHOD

F: Forced

S: Scheduled

\section{A-Equipment Failure}

B-Maintenance or Test

C-Refuel ing

D-Regulatory Restriction

E-Operator Training \&

License Examination

F-Administrative

G-Operational Error

$H$-other

1-Manual
2-Manual Scram
3-Auto Scram
4-Cont inued
5-Reduced Load
9-0ther

\section{SYSTEM}

IEEE Standard

NUREG-0161 Exhibit F

\section{COMPONENT}

\section{IEEE Standard}

803A-1983 and/or

MUREG-0161 Exhibit 


\section{FACILITY DESCRIPTION}

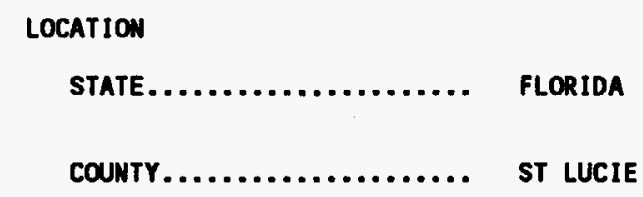

TYPE OF REACTOR.

PUR

DATE INITIAL CRITICALITY.

DATE INITIAL ELECTRICITY

DATE COMMERCIAL OPERATE.

CONDENSER COOLING METHOD

CONDENSER COOLING WATER

ELECTRIC RELIABILITY

COUNCIL.................. SOUTHEASTERN ELECTRIC

RELIABILITY COUNCIL

\section{UTILITY \& CONTRACTOR INFORMATION}

UTILITY

LICENSEE................ FLORIDA POWER \& LIGHT CO.

CORPORATE ADDRESS......... 9250 MEST FLAGLER STREET MIAMI, FLORIDA 33102

\section{CONTRACTOR}

ARCHITECT/ENGINEER.......... EBASCO

NUC STEAM SYS SUPPLIER...... COMBUSTION ENGINEERING

CONSTRUCTOR

EBAsco

TURBINE SUPPLIER

WEST INGHOUSE

\section{REGULATORY INFORMATION}

IE REGION RESPONSIBLE......... 2

IE RESIDENT INSPECTOR.......... RICHARD PREVATTE

LICENSING PROJ MAMAGER......... JAN ADAM NORRIS

DOCKET MUMBER............... 50-389

LICENSE \& DATE ISSUANCE........ NPF 016, JUNE 10, 1983 
:5270\%

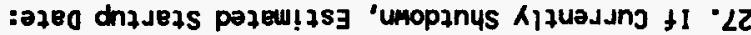

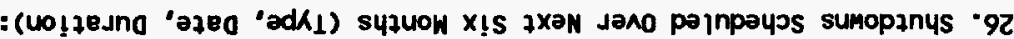

\begin{tabular}{|c|c|}
\hline $\mathbf{\Sigma} 06$ & IE \\
\hline 506 & OE \\
\hline 188 & 62 \\
\hline 658 & 82 \\
\hline 258 & $\angle 2$ \\
\hline SLL & 92 \\
\hline $0 \gg 2$ & $\mathbb{S 2}$ \\
\hline 109 & 72 \\
\hline 679 & $\Sigma 2$ \\
\hline 927 & 22 \\
\hline I8E & 12 \\
\hline I8E & 02 \\
\hline OBE & 61 \\
\hline$\angle 9 \varepsilon$ & 81 \\
\hline$S \angle 2$ & $\angle l$ \\
\hline S9l & $9 l$ \\
\hline
\end{tabular}

\begin{tabular}{|c|c|}
\hline$t \Sigma-$ & sl \\
\hline 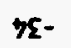 & t \\
\hline $7 \sqrt{2}-$ & El \\
\hline SE- & $\mathrm{Zl}$ \\
\hline OZ- & แ \\
\hline Sl- & 01 \\
\hline Sl- & 6 \\
\hline $21-$ & 8 \\
\hline $21-$ & $L$ \\
\hline S2- & 9 \\
\hline SE- & $\boldsymbol{5}$ \\
\hline SE- & $\eta$ \\
\hline ZE- & $\boldsymbol{\varepsilon}$ \\
\hline 81- & 2 \\
\hline LZ- & I \\
\hline yanod & $A \forall 0$ \\
\hline
\end{tabular}

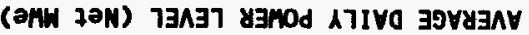

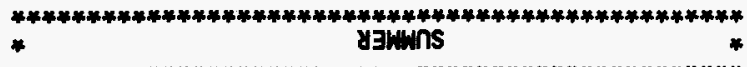

\begin{tabular}{|c|c|c|}
\hline $9^{\circ} 900^{\prime} \downarrow$ & 0.0 & 0.0 \\
\hline l.5 & 0.0 & 0.0 \\
\hline $0=i L$ & $\varepsilon=95$ & $L=2 \Sigma$ \\
\hline $2 \cdot 22$ & $\varepsilon 25$ & E. ES \\
\hline $1 \cdot 82$ & 8.89 & 5.15 \\
\hline $1-8 L$ & 8.89 & $\varepsilon^{\circ} 15$ \\
\hline 0.650'509'19 & 0-92ち‘85ち’† & $0.92 L^{\prime} 612$ \\
\hline$\overline{0.627^{\prime}+28^{\prime}+9}$ & $0.009^{\circ} 10 L^{\prime} t$ & $0^{\circ} 095^{\prime} 272$ \\
\hline $0^{\circ} 960^{\prime} 155^{\prime} 561$ & $0^{\circ} 50 l^{\prime} 252^{\prime} \mapsto \mathrm{l}$ & 0.9عl'92L \\
\hline 0.0 & 0.0 & 0.0 \\
\hline$S^{\prime} 20 \varepsilon^{\prime} S L$ & l. $220 ' 9$ & $8^{\circ} 185$ \\
\hline 0.0 & 0.0 & 0.0 \\
\hline $0.929^{\prime} 92$ & 5.060'9 & $5 \cdot 127$ \\
\hline 0.257'96 & $0.09 L^{\prime} 8$ & 0.572 \\
\hline כณII & y४ & HLHOW \\
\hline
\end{tabular}

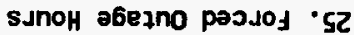

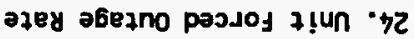
(zON 830) J0708g dej 7 ! (дәN JOW) JOzJeg dej z! Un 'Z2 jozogy j!eay 7 !un $\cdot 12$

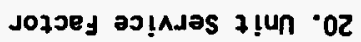
(HNW) Jaug 2013 7อN 61 (HNW) JaU गอ1] SSOJg - 81

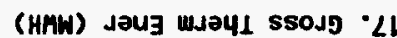

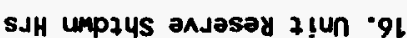
ou! 7-40 Jozejauag SJH S.H U4p74S әA.jesey $x y \cdot+1$

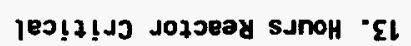

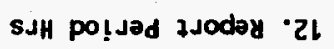

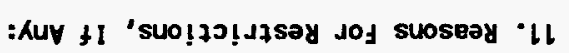

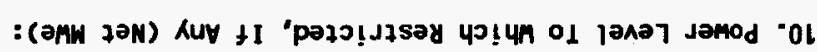

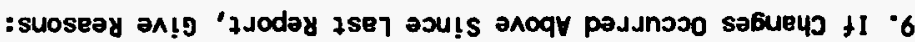

\begin{tabular}{l}
\hline 588 \\
\hline 226 \\
\hline 006 \\
\hline 006 \\
\hline $5 L 12$ \\
\hline
\end{tabular}

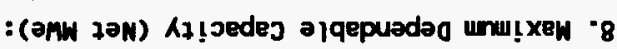

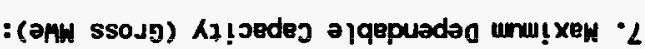

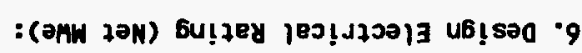
: (әHN sso.j) 6u!fey әfejdeuen 's : (7NK) Janod jewsayl pasuas!

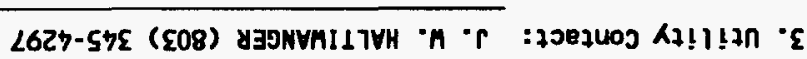

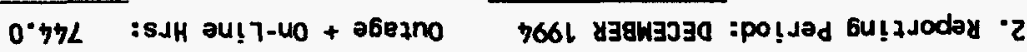

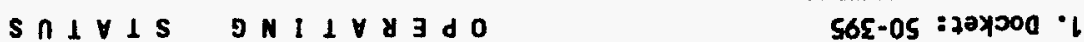




No. Date Type Hours Reason Method LER Number System Component Cause and Corrective Action To Prevent Recurrence

TYPE

F: Forced

S: Scheduled

\begin{tabular}{l} 
REASON \\
\hline A-Equipment Fai lure \\
B-Maintenance or Test \\
C-Refuel ing \\
D-Regulatory Restriction \\
E-Operator Training \& \\
L-icense Examination \\
F-Adninistrative \\
G-Operational Error \\
H-Other.
\end{tabular}

METHOD

1-Manua

2-Manual Scram

3-Auto Screm

3-Cont inued

5-Reduced Load

9-0ther
SYSTEM

IEEE Standard

NUREG-0161
COMPONENT

IEEE Standard

803A-1983 and/or

NUREG-0161 Exhibit H

F-Administrative

$H$-other 


\section{FACILITY OESCRIPTION}

\section{LOCATION}

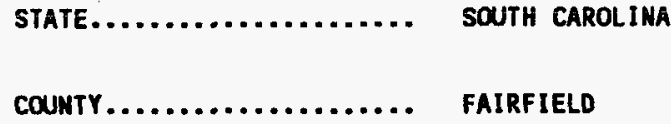

UTILITY \& CONTRACTOR INFORMATION

\section{UTILITY}

L.ICENSEE.

SOUTH CAROLINA ELECTRIC \& GAS CO.

CORPORATE ADDRESS

P. 0. BOX 764

COLUMBIA, SOUTH CAROLIMA 29218

\section{CONTRACTOR}

ARCHITECT/ENGINEER.

GILBERT ASSOCIATES

NUC STEAM SYS SUPPLIER...... VESTINGHOUSE

CONSTRUCTOR............... DANIEL INTERMATIONAL

TURBIME SUPPLIER........... GENERAL ELECTRIC

\section{REGULATORY INFORMATION}

IE REGION RESPONSIBLE........ 2

IE RESIDENT INSPECTOR......... ROBERT HAAE

LICENSING PROJ MANAGER......... GEORGE F. WUNDER

DOCKET MUMBER............. 50-395

LICENSE \& DATE ISSUANCE........ NPF 012, NOVEMBER 12, 1982 
1. Docket: $50-280$

OPERATING STATUS

2. Reporting Period: DECEMBER 1994

Outage + On-Line Hrs: 744.0

3. Utility Contact: D. MASON (804) 365-2459

4. Licensed Thermal Power (MWt):

5. Nameplate Rating (Gross MWe):

6. Design Electrical Rating (Net MWe):

7. Maximum Dependable Capacity (Gross MWe):

8. Maximum Dependable Capacity (Net MHe):

9. If Changes Occurred Above Since Last Report, Give Reasons:

10. Power Level To Which Restricted, If Any (Net MWe):

11. Reasons For Restrictions, If Any:

\begin{tabular}{|c|c|c|c|}
\hline & MONTH & YEAR & CUMULATIVE \\
\hline 12. Report Period Hrs & 744.0 & $8,760.0$ & $193,080.0$ \\
\hline 13. Hours Reactor Critical & 215.4 & $6,662.5$ & $130,469.7$ \\
\hline 14. Rx Reserve Shtdwn Hrs & 0.0 & 0.0 & $3,774.5$ \\
\hline 15. Hrs Generator On-Line & 152.3 & $6,562.0$ & $128,241.0$ \\
\hline 16. Unit Reserve Shtdun Hrs & 0.0 & 0.0 & $3,736.2$ \\
\hline 17. Gross Therm Ener (MUH) & $331,175.4$ & $15,285,994.1$ & $298,453,739.2$ \\
\hline 18. Gross Elec Ener (MWH) & $110,500.0$ & $5,074,285.0$ & $97,613,833.0$ \\
\hline 19. Net Elec Ener (MWH) & $106,379.0$ & $4,881,922.0$ & $92,709,023.0$ \\
\hline 20. Unit Service Factor & 20.5 & 74.9 & 66.4 \\
\hline 21. Unit Avail Factor & 20.5 & 74.9 & 68.4 \\
\hline 22. Unit Cap Factor (MDC Net) & 18.3 & 71.4 & 61.9 \\
\hline 23. Unit Cap Factor (DER Net) & 18.1 & 70.7 & 60.9 \\
\hline 24. Unit Forced Outage Rate & 0.0 & 0.4 & 16.7 \\
\hline 25. Forced Outage Hours & 0.0 & 26.4 & $25,731.2$ \\
\hline
\end{tabular}

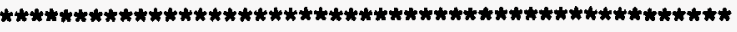

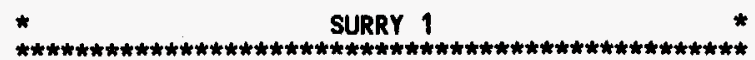

AVERAGE DAILY POWER LEVEL (Net MWe)

\begin{tabular}{lr} 
DAY & PONER \\
\hline 2 & 0 \\
\hline 3 & 0 \\
\hline 4 & 0 \\
\hline 5 & 0 \\
\hline 6 & 0 \\
\hline 7 & 0 \\
\hline 8 & 0 \\
\hline 9 & 0 \\
\hline 10 & 0 \\
\hline 11 & 0 \\
\hline 12 & 0 \\
\hline 13 & 0 \\
\hline 14 & 0 \\
\hline 15 & 0 \\
\hline
\end{tabular}

\begin{tabular}{cc} 
DAY & PONER \\
\hline 16 & 0 \\
\hline 17 & 0 \\
\hline 18 & 0 \\
\hline 19 & 0 \\
\hline 20 & 0 \\
\hline 21 & 0 \\
\hline 22 & 0 \\
\hline 23 & 0 \\
\hline 24 & 0 \\
\hline 25 & 70 \\
\hline 26 & 488 \\
\hline 27 & 767 \\
\hline 28 & 775 \\
\hline 29 & 742 \\
\hline 30 & 795 \\
\hline 31 & 795 \\
\hline
\end{tabular}

26. Shutdowns Scheduled Over Next six Months (Type, Date, Duration):

27. If Currently Shutdown, Estimated Startup Date:

Notes:

CUMULATIVE UNIT CAPACITY FACTOR (MDC NET) IS CALCULATED WITH A WEIGHTED AVERAGE. 


\begin{tabular}{|c|c|c|c|c|c|c|c|c|c|}
\hline Mo. & Date & Type & Hours & Reason & Method & LER Number & System & Component & Cause and Corrective Action To Prevent Recurrence \\
\hline & $11 / 28 / 94$ & $\mathbf{s}$ & 591.7 & B & 4 & & JB & SG & MAINTENANCE OUTAGE CONTINUED. \\
\hline & $12 / 29 / 94$ & $\mathbf{F}$ & 0.0 & A & 5 & & JD & RCT & $\begin{array}{l}\text { TURBINE RUNBACK INITIATED WHEN IRPI K-02 FAILED. } \\
\text { IRPI K-02 INSTRUMENTATION CABLE WAS REPLACEDD. }\end{array}$ \\
\hline
\end{tabular}

\section{TYPE}

F: Forced

S: Scheduled

\section{REASON}

A-Equipment failure B-Maintenance or Test C-Refuel ing

D-Regulatory Restriction

E-Operator Training \&

License Examination

F-Adninistrative

G-Operational Error

H-Other

\begin{tabular}{l} 
METHOD \\
\hline 1-Manual \\
2-Manual Scram \\
3-Auto Scran \\
4-Cont inued \\
5-Reduced Load \\
9-0ther
\end{tabular}

SYSTEM

IEEE Standard

NUREG-0161 Exhibit F
COMPONENT

IEEE Standard

803A-1983 and/or

MUREG-0161 Exhibit H 


\section{FACILITY DESCRIPTION}

\section{LOCATION}

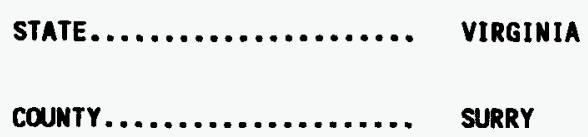
DIST AND DIRECTION FRON
NEAREST POPULATION CTR...

TYPE OF REACTOR

17 MI NH OF MEUPORT MEUS, VA

DATE IMITIAL CRITICALITY...... JULY 01, 1972

DATE INITIAL ELECTRICITY...... JULY 04, 1972

DATE COMMERCIAL OPERATE....... DECEMBER 22, 1972

CONDENSER COOLING METHOD...... ONCE THRU

CONDENSER COOLING WATER....... JAMES RIVER

ELECTRIC RELIABILITY

COUNCIL................. SOUTHEASTERN ELECTRIC RELIABILITY COUNCIL

\section{UTILITY \& CONTRACTOR INFORMATION}

UTILITY

LICENSEE

CORPORATE ADDRESS

VIRGINIA ELECTRIC \& POWER CO.

P.0. BOX 26666

RICHMOND, VIRGINIA 23261

\section{CONTRACTOR}

ARCHI TECT/ENGINEER.

STONE \& WEBSTER

NUC STEAM SYS SUPPLIER

WESTINGHOUSE

CONSTRUCTOR

STONE \& WEBSTER

TURBINE SUPPLIER

WEST INGHOUSE

RECULATORY INFORMATION

IE REGION RESPONSIBLE......... 2

IE RESIDENT INSPECTOR......... MORRIS BRANCH

LICEMSING PROJ MANAGER........ BARTHOLONEY C. BUCKLEY

DOCKET MUMBER.............. $50-280$

LICENSE \& DATE ISSUANCE........ DPR 032, MAY 25, 1972 
1. Docket: $50-281$

OPERATING STATUS

2. Reporting Period: DECEMBER 1994

Outage + On-Line Hrs: $\quad 744.0$

3. Utility Contact: D. MASON (804) 365-2459

4. Licensed Thermal Power (MUt):

5. Nameplate Rating (Gross MHe):

6. Design Electrical Rating (Net MHe):

7. Maximum Dependable Capacity (Gross MHe):

8. Maximum Dependable Capacity (Net MWe):

9. If Changes Occurred Above Since Last Report, Give Reasons:

10. Power Level To Which Restricted, If Any (Net MHe):

11. Reasons For Restrictions, If Any:

\begin{tabular}{|c|c|c|c|}
\hline & MONTH & YEAR & CUMULATIVE \\
\hline 12. Report Period Hrs & 744.0 & $8,760.0$ & $189,960.0$ \\
\hline 13. Hours Reactor Critical & 744.0 & $8,261.2$ & $128,337.5$ \\
\hline 14. Rx Reserve Shtohn Hrs & 0.0 & 0.0 & 328.1 \\
\hline 15. Hrs Generator On-Line & 744.0 & $8,251.5$ & $126,467.5$ \\
\hline 16. Unit Reserve Shtdwn Hrs & 0.0 & 0.0 & 0.0 \\
\hline 17. Gross Therm Ener (MUH) & $1,814,475.4$ & $19,592,721.9$ & $295,268,464.3$ \\
\hline 18. Gross Elec Ener (MWH) & $605,405.0$ & $6,496,670.0$ & $96,442,294.0$ \\
\hline 19. Net Elec Ener (MUH) & $584,305.0$ & $6,260,966.0$ & $91,593,036.0$ \\
\hline 20. Unit Service Factor & 100.0 & 94.2 & 66.6 \\
\hline 21. Unit Avail Factor & 100.0 & 94.2 & 66.6 \\
\hline 22. Unit Cap Factor (MDC Net) & 100.6 & 91.5 & 61.8 \\
\hline 23. Unit Cap Factor (DER Net) & 99.7 & 90.7 & 61.2 \\
\hline 24. Unit forced Outage Rate & 0.0 & 0.0 & 13.3 \\
\hline 25. Forced Outage Hours & 0.0 & 0.0 & 19.418 .8 \\
\hline
\end{tabular}

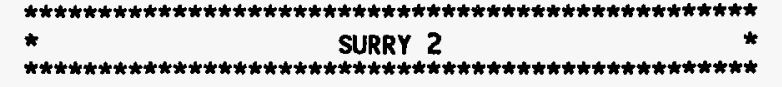

AVERAGe DAILY POWER LeVel (Net MWe)

\begin{tabular}{lc} 
DAY & POWER \\
\hline & 787 \\
\hline 2 & 787 \\
\hline 3 & 789 \\
\hline 4 & 790 \\
\hline 5 & 783 \\
\hline 6 & 783 \\
\hline 7 & 789 \\
\hline 8 & 790 \\
\hline 9 & 790 \\
\hline 10 & 789 \\
\hline 11 & 790 \\
\hline 12 & 785 \\
\hline 13 & 782 \\
\hline 14 & 786 \\
\hline 15 & 786 \\
\hline
\end{tabular}

\begin{tabular}{cc} 
DAY & POWER \\
\hline 16 & 787 \\
\hline 17 & 786 \\
\hline 18 & 786 \\
\hline 19 & 786 \\
\hline 20 & 785 \\
\hline 21 & 784 \\
\hline 22 & 784 \\
\hline 23 & 784 \\
\hline 24 & 785 \\
\hline 25 & 785 \\
\hline 26 & 785 \\
\hline 27 & 782 \\
\hline 28 & 763 \\
\hline 29 & 785 \\
\hline 30 & 786 \\
\hline 31 & 787 \\
\hline
\end{tabular}

26. Shutdowns Scheduled Over Next six Months (Type, Date, Duration): REFUELING OUTAGE, FEBRUARY 2, 1995, 53 DAYS.

27. If Currently Shutdown, Estimated Startup Date: Notes:

CUMULATIVE UNIT CAPACITY FACTOR (MDC NET) IS CALCULATED WITH A WEIGHTED AVERAGE. 


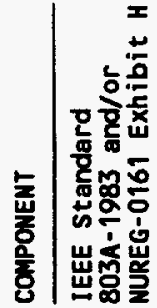

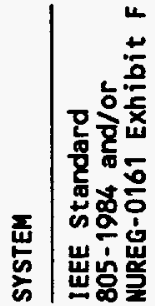

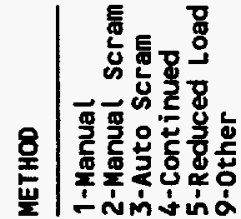

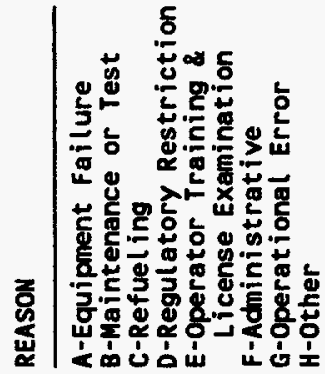

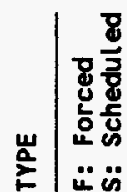




\section{FACILITY DESCRIPTION}

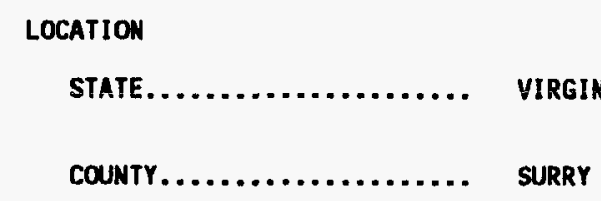

\section{DIST AND DIRECTION FRON} MEAREST POPULATION CTR.

TYPE OF REACTOR.

17 MI NH OF MEHPORT MEUS, VA

PWR

DATE INITIAL CRITICALITY...... MARCH 07, 1973

DATE INITIAL ELECTRICITY...... MARCH 10, 1973

DATE COMMERCIAL OPERATE....... maY 01, 1973

CONDENSER COOLING METHOD...... ONCE THRU

CONDENSER COOLING WATER....... JAMES RIVER

ELECTRIC RELIABILITY

COUNCIL................. SOUTHEASTERN ELECTRIC RELIABILITY COUNCIL

\section{UTILITY \& CONTRACTOR INFORMATION}

UTILITY

LICENSEE

CORPORATE ADDRESS.......... P.O. BOX 26666

RICHMOND, VIRGINIA 23261

\section{CONTRACTOR}

ARCHI TECT/ENGINEER.

STONE \& WEBSTER

NUC STEAM SYS SUPPLIER.

WESTIMGHOUSE

CONSTRUCTOR.

STONE \& MEBSTER

TURBINE SUPPLIER........... WESTINGHOUSE

\section{REGULATORY INFORMATION}

IE REGION RESPONSIBLE......... 2

IE RESIDENT INSPECTOR.......... MORRIS BRANCH

LICENSING PROJ MAMAGER......... BARTHOLOMEW C. BUCKLEY

DOCKET MUMBER.............. 50-281

LICENSE \& DATE ISSUANCE. 
1. Docket: $50-387$

DPERATING STATUS

2. Reporting Period: DECEMBER 1994

Outage + On-Line Hrs: $\quad 744.0$

3. Utility Contact: R. S. BALL (717) 542-3453

4. Licensed Thermal Power (MWt):

5. Nameplate Rating (Gross Mue):

6. Design Electrical Rating (Net MWe):

7. Maximum Dependable Capacity (Gross MWe):

8. Maximum Dependable Capacity (Net MHe):

\begin{tabular}{c}
\hline 3293 \\
\hline 1152 \\
\hline 1050 \\
\hline 1078 \\
\hline 1040
\end{tabular}

9. If Changes Occurred Above Since Last Report, Give Reasons:

10. Power Level To Which Restricted, If Any (Net MWe):

11. Reasons For Restrictions, If Any:

\begin{tabular}{|c|c|c|c|}
\hline & MONTH & YEAR & CUMULATIVE \\
\hline 12. Report Period Mrs & 744.0 & $8,760.0$ & $101,401.0$ \\
\hline 13. Hours Reactor Critical & 744.0 & $8,292.4$ & $79,240.9$ \\
\hline 14. Rx Reserve Shtchn Hrs & 0.0 & 0.0 & $1,032.0$ \\
\hline 15. Hrs Generator On-Line & 744.0 & $8,249.5$ & 77.754 .8 \\
\hline 16. Unit Reserve Shtdin Hrs & 0.0 & 0.0 & 0.0 \\
\hline 17. Gross Therm Ener (MUH) & $2,421,800.0$ & $26,671,352.0$ & $245,157,870.0$ \\
\hline 18. Gross Elec Ener (MUH) & $802,852.0$ & $8,728,886.0$ & $80,083,220.0$ \\
\hline 19. Net Elec Ener (NHH) & $775,577.0$ & $8,414,452.0$ & $76,976,960.0$ \\
\hline 20. Unit Service Factor & 100.0 & 94.2 & 76.7 \\
\hline 21. Unit Avail Factor & 100.0 & 94.2 & 76.7 \\
\hline 22. Unit Cap Factor (MDC Net) & 100.2 & 92.4 & 73.0 \\
\hline 23. Unit Cap Factor (DER Net) & 99.3 & 91.5 & 72.3 \\
\hline 24. Unit Forced Outage Rate & 0.0 & 0.0 & 7.7 \\
\hline 25. Forced Outage Hours & 0.0 & 0.0 & $6,441.1$ \\
\hline
\end{tabular}

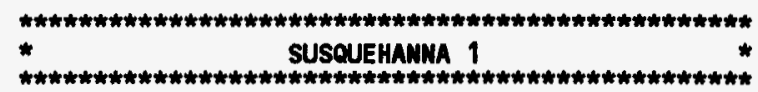

AVERAGE DAILY PONER LEVEL (Net MUe)

\begin{tabular}{ll} 
DAY & PONER \\
\hline 1 & 1054 \\
\hline 2 & 1058 \\
\hline 3 & 1058 \\
\hline 4 & 1055 \\
\hline 5 & 1048 \\
\hline 6 & 1049 \\
\hline 7 & 1056 \\
\hline 8 & 1061 \\
\hline 9 & 1059 \\
\hline 10 & 1057 \\
\hline 11 & 1057 \\
\hline 12 & 1060 \\
\hline 13 & 1056 \\
\hline 14 & 1056 \\
\hline 15 & 1058 \\
\hline
\end{tabular}

\begin{tabular}{cc} 
DAY & PONER \\
\hline 16 & 1007 \\
\hline 17 & 680 \\
\hline 18 & 1047 \\
\hline 19 & 1058 \\
\hline 20 & 1058 \\
\hline 21 & 1057 \\
\hline 22 & 1056 \\
\hline 23 & 1056 \\
\hline 24 & 1056 \\
\hline 25 & 1057 \\
\hline 26 & 1057 \\
\hline 27 & 1058 \\
\hline 28 & 1056 \\
\hline 29 & 1057 \\
\hline 30 & 1058 \\
\hline 31 & 1056 \\
\hline
\end{tabular}

26. Shutdowns Scheduled Over Mext Six Months (Type, Date, Duration): REFUELING OUTAGE, MARCH 25, 1995, 50 DAYS.

27. If Currently Shutdown, Estimated Startup Date:

Notes:

LICENSEE REVISED JANUARY 1994 GROSS THERMAL ENERGY FROM $582,578.0$ TO $503,515.0$ 


\begin{tabular}{|c|c|c|c|c|c|c|c|c|c|}
\hline No. & Date & Type & Hours & Reason & Method & LER Number & System & Component & Cause and Corrective Action To Prevent Recurrence \\
\hline 9 & $12 / 16 / 94$ & $\mathbf{s}$ & 0.0 & B & 5 & & $\mathbf{x x}$ & 222 & $\begin{array}{l}\text { PONER REDUCTION TO PERFORM A CONTROL ROD SEQUENCE } \\
\text { EXCHANGE. OTHER WORK PERFORMED DURING THE } \\
\text { DOWHPONER INCLUDED REACTOR RECIRC MG SET BRUSH } \\
\text { CHANGEOUT AND SCRAM TIME TESTING. }\end{array}$ \\
\hline
\end{tabular}

\begin{tabular}{l} 
TYPE \\
\hline F: Forced \\
S: Scheduled
\end{tabular}

\section{REASON}

A-Equipment Failure
B-Maintenance or Test

B-Maintenance
C-Refuel ing

D-Regulatory Restriction

E-Operator Training \&

License Examination

F-Administrative

G-Operational Error

$\mathrm{H}$-other

\section{SYSTEM}

1-Manual

2-Manual Scran

3-Auto Scram

4-Cont inued

5-Reduced Load

9-0ther
IEEE Standard

805-1984 and/or
NUREG-0161 Exhibit

\section{COMPONENT}

IEEE Standard

NUREG-0161 Exhibit H 


\section{FACILITY DESCRIPTION}

\section{LOCATION}

STATE

county

PENNSYlvania

LUZERNE

\section{DIST AND DIRECTION FROM}

MEAREST POPULATION CTR..... 7 MI NE OF BERHICK, PA

TYPE OF REACTOR.

BUR

DATE InITIAL CRITICALITY...... SEPTEMBER 10, 1982

DATE INITIAL ELECTRICITY...... NOVEMBER 16, 1982

date COMnERCIAL OPERATE....... JUNE 08, 1983

CONDENSER COOLING METHOD...... CC, HNDCT

CONDENSER COOLING WATER........ SUSQUEHANMA RIVER

ELECTRIC RELIABILITY

COUNCIL................. MID-ATLANTIC AREA COUNCIL

\section{UTILITY \& CONTRACTOR INFORMATION}

UTILITY

Licensee................. Pennsylvania POWER \& Light co.

CORPORATE ADDRESS......... 2 NORTH NINTH STREET ALLENTOWN, PENNSYLVANIA 18101

CONTRACTOR

ARCHI TECT/ENGINEER.......... BECHTEL

MUC STEAM SYS SUPPLIER...... GENERAL ELECTRIC

CONSTRUCTOR.............. BECHTEL

TURBINE SUPPLIER.......... GENERAL ELECTRIC

REGULATORY INFORMATION

IE REGION RESPONSIBLE......... 1

IE RESIDENT INSPECTOR......... MAITRI BAMERJEE

LICENSING PROJ MANAGER........ CHESTER POSLUSNY JR.

DOCKET MUMBER.............. 50-387

LICENSE \& DATE ISSUANCE........ MPF 014, NOVEMBER 12, 1982 


\section{Docket: $50-388$}

OPERATING STATUS

2. Reporting Period: DECEMBER 1994

outage + On-Line Hrs:

744.0

3. Utility Contact: R. S. BALL (717) 542-3453

4. Licensed Thermal Power (mut):

5. Nameplate Rating (Gross Me):

6. Design Electrical Rating (Net Mue):

7. Maximum Dependable Capacity (Gross MUe):

8. Maximum Dependable Capacity (Net MWe):

9. If Changes Occurred Above Since Last Report, Give Reasons:

10. Power Level To thich Restricted, If Any (Net MHe):

11. Reasons for Restrictions, If Any:

\begin{tabular}{|c|c|c|c|}
\hline & MONTH & YEAR & CUIMLAT IVE \\
\hline 12. Report Period Hrs & 744.0 & $8,760.0$ & $86,640.0$ \\
\hline 13. Hours Reactor Critical & 744.0 & $6,673.8$ & $72,187.0$ \\
\hline 14. RX Reserve Shtchn Hrs & 0.0 & 0.0 & 717.9 \\
\hline 15. Hrs Generator on-Line & 744.0 & $6,579.1$ & $70,790.8$ \\
\hline 16. Unit Reserve Shtdun Hrs & 0.0 & 0.0 & 0.0 \\
\hline 17. Gross Therm Ener (MUH) & $2,549,705.0$ & $21,715,080.0$ & $226,042,710.0$ \\
\hline 18. Gross Elec Ener (MUH) & $850,134.0$ & $7,165,017.0$ & $74,174,784.0$ \\
\hline 19. Net Elec Ener (MUH) & $821,586.0$ & $6,895,720.0$ & $71,400,895.0$ \\
\hline 20. Unit Service Factor & 100.0 & 75.1 & 81.7 \\
\hline 21. Unit Avail Factor & 100.0 & 75.1 & 81.7 \\
\hline 22. Unit Cap Factor (MDC Net) & 100.9 & 72.8 & 78.7 \\
\hline 23. Unit Cap Factor (DER Net) & 100.4 & 72.4 & 78.2 \\
\hline 24. Unit Forced Outage Rate & 0.0 & 0.8 & 5.3 \\
\hline 25. Forced Outage Hours & 0.0 & 56.0 & $3,940.6$ \\
\hline
\end{tabular}

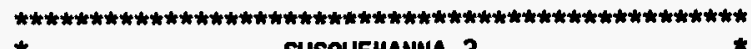

SUSQUEHANNA 2 P

AVERAGe DAILY PONER LEVEL (Net MWe)

\begin{tabular}{ll} 
DAY & PONER \\
\hline 1 & 1110 \\
\hline 2 & 1074 \\
\hline 3 & 1056 \\
\hline 4 & 1105 \\
\hline 5 & 1101 \\
\hline 6 & 1101 \\
\hline 7 & 1105 \\
\hline 8 & 1109 \\
\hline 9 & 1108 \\
\hline 10 & 1109 \\
\hline 11 & 1107 \\
\hline 12 & 1109 \\
\hline 13 & 1108 \\
\hline 14 & 1111 \\
\hline 15 & 1110 \\
\hline
\end{tabular}

\begin{tabular}{ll} 
DAY & PONER \\
\hline 16 & 1111 \\
\hline 17 & 1109 \\
\hline 18 & 1109 \\
\hline 19 & 1110 \\
\hline 20 & 1110 \\
\hline 21 & 1110 \\
\hline 22 & 1110 \\
\hline 23 & 1109 \\
\hline 24 & 1106 \\
\hline 25 & 1108 \\
\hline 26 & 1108 \\
\hline 27 & 1108 \\
\hline 28 & 1108 \\
\hline 29 & 1109 \\
\hline 30 & 1110 \\
\hline 31 & 1074 \\
\hline
\end{tabular}

26. Shutdowns Scheduled Over Mext Six Months (Type, Date, Duration):

27. If Currently Shutdown, Estimated Startup Date:

Notes:

YEAR-TO-DATE AMD CUMULATIVE UNIT CAPACITY FACTORS (MDC \& DER) ARE CALCULATED WITH WEIGHTED AVERAGES. LICENSEE REVISED JULY 1994 GROSS THERMAL EMERGY FROM 2,446,455.0 TO 2,556,408.0 AMD AUGUST 1994 GROSS THERMAL ENERGY FROM 2,411,674.0 TO $2,520,064.0$. 


$\frac{\text { TYPE }}{\text { F: Forced }}$

\section{REASON}

A-Equipment Failure

A-Equipment Failure

C-Refuel ing

D-Regulatory Restriction

E-Operator Training 8

License Examination

F-Administrat ive

G-Operational Error

H-other
METHOD

\begin{tabular}{l} 
METHOD \\
\hline 1-Manual \\
2-Manual Scram \\
3-Auto Scram \\
4-Cont inued \\
5-Reduced Load \\
9-0ther
\end{tabular}

SYSTEM

IEEE Standard

NUREG-0161 Exhibit $F$

COMPONENT

IEEE Standard

803A-1983 and/or

MUREG-0161 Exhibit 
FACILITY DESCRIPTION

LOCATION

STATE

COUNTY

PENMSYLVANIA

LUZERNE

DIST AND DIRECTION FROM

NEAREST POPULAT

7 MI NE OF BERHICK, PA

TYPE OF REACTOR

BWR

DATE INITIAL CRITICALITY...... MAY 08, 1984

DATE INITIAL ELECTRICITY...... JULY 03, 1984

DATE COMMERCIAL OPERATE....... FEBRUARY 12, 1985

CONDENSER COOLING METHOD....... CC, HNDCT

COMDENSER COOLING WATER....... SUSQUEHANNA RIVER

ELECTRIC RELIABILITY

COUNCIL.................... MID-ATLANTIC AREA COUHCIL

\section{UTILITY \& CONTRACTOR INFORMATION}

\section{UTILITY}

LICENSEE.

CORPORATE ADDRESS.

PENNSylvania PONER \& Light CO.

\section{NORTH NINTH STREET}

ALLENTON, PENNSYLVANIA 18101

CONTRACTOR

ARCHITECT/ENGINEER......... BECHTEL

NUC STEAM SYS SUPPLIER...... GENERAL ELECTRIC

CONSTRUCTOR.............. BECHTEL

TURBINE SUPPLIER.......... GEMERAL ELECTRIC

\section{REGULATORY INFORMATION}

IE REGION RESPONSIBLE......... 1

IE RESIDENT INSPECTOR........ MAITRI BAMERJEE

LICENSING PROJ MANAGER......... CHESTER POSLUSNY JR.

DOCKET MUMBER.............. 50-388

LICENSE \& DATE ISSUANCE........ MPF 022, JUNE 27, 1984 
1. Docket: $50-289$

OPERATING STATUS

2. Reporting Period: DECEMBER 1994

Outage + On-Line Hrs: $\quad 744.0$

3. Utility Contact: H. HEYSEK (717) $948-8191$

4. Licensed Thermal Power (MUt):

5. Nameplate Rating (Gross MWe):

6. Design Electrical Rating (Net MWe):

7. Maximm Dependable Capacity (Gross MHe):

8. Maximum Dependable Capacity (Net MWe):

9. If Changes Occurred Above Since Last Report, Give Reasons:

10. Power Level To Which Restricted, If Any (Net MWe):

11. Reasons for Restrictions, If Any:

MONTH YEAR CUMULATIVE

12. Report Period Hrs

13. Hours Reactor Critical

14. Rx Reserve Shtdwn Hrs

15. Hrs Generator On-Line

16. Unit Reserve Shtdwn Hrs

17. Gross Therm Ener (MHH)

18. Gross Elec Ener (MUH)

19. Net Elec Ener (MWH)

20. Unit Service Factor

21. Unit Avail factor

22. Unit Cap Factor (MDC Net)

23. Unit Cap Factor (DER Net)

24. Unit Forced Outage Rate

25. Forced Outage Hours

$\begin{array}{r}\text { MONTH } \\ \hline 744.0 \\ \hline 744.0 \\ \hline 0.0 \\ \hline 744.0 \\ \hline 0.0 \\ \hline 621,696.0 \\ \hline 586,496.0 \\ \hline 100.0 \\ \hline 100.0 \\ \hline 100.3 \\ \hline 96.3 \\ \hline 0.0 \\ \hline 0.0 \\ \hline\end{array}$

\begin{tabular}{|c|c|c|c|}
\hline & 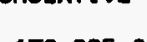 & 10 & 819 \\
\hline $8,100.0$ & $178,2<3.0$ & 11 & 818 \\
\hline $8,362.6$ & $101,588.8$ & 12 & 823 \\
\hline 0.0 & $2,283.8$ & 13 & 822 \\
\hline $8,350.5$ & $100,454.4$ & 14 & 821 \\
\hline 0.0 & 0.0 & 15 & 820 \\
\hline
\end{tabular}

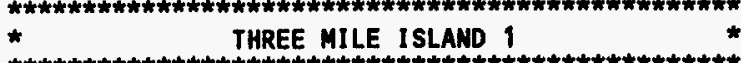

AVERAGE DAILY POWER LEVEL (Net MWe)

\begin{tabular}{ll} 
DAY & POWER \\
\hline & 816 \\
\hline 2 & 814 \\
\hline 3 & 744 \\
\hline 4 & 355 \\
\hline 5 & 429 \\
\hline 6 & 802 \\
\hline 7 & 808 \\
\hline 8 & 819 \\
\hline 9 & 821 \\
\hline 10 & 819 \\
\hline 11 & 818 \\
\hline 12 & 823 \\
\hline 13 & 822 \\
\hline 14 & 821 \\
\hline 15 & 820 \\
\hline
\end{tabular}

\begin{tabular}{ll} 
DAY & PONER \\
\hline 16 & 819 \\
\hline 17 & 818 \\
\hline 18 & 818 \\
\hline 19 & 819 \\
\hline 20 & 820 \\
\hline 21 & 818 \\
\hline 22 & 819 \\
\hline 23 & 816 \\
\hline 24 & 815 \\
\hline 25 & 815 \\
\hline 26 & 818 \\
\hline 27 & 820 \\
\hline 28 & 821 \\
\hline 29 & 821 \\
\hline 30 & 826 \\
\hline 31 & 825 \\
\hline
\end{tabular}

26. Shutdowns Scheduled Over Next six Months (Type, Date, Duration):

27. If Currently Shutdown, Estimated Startup Date:

Notes: 


\begin{tabular}{|c|c|c|c|c|c|c|c|c|c|}
\hline No. & Date & Type & Hours & Reason & Method & LER Number & system. & Component & Cause and Corrective Action To Prevent Recurrence \\
\hline $94-08$ & $12 / 03 / 94$ & $\mathbf{F}$ & 0.0 & A & 5 & & SG & COND & $\begin{array}{l}\text { A CHEMISTRY ANOMALY INDICATING A MAIN CONDENSER } \\
\text { TUBE LEAK CAUSED A REDUCTION IN POWER TO EFFECT } \\
\text { REPAIRS TO THE MAIN CONDEMSER. }\end{array}$ \\
\hline
\end{tabular}

\begin{tabular}{|c|c|}
\hline TYPE & REASON \\
\hline $\begin{array}{l}\text { F: Forced } \\
\text { S: Scheduled }\end{array}$ & $\begin{array}{l}\text { A-Equipment Failure } \\
\text { B-Maintenance or Test } \\
\text { C-Refuel ing } \\
\text { D-Regulatory Restriction } \\
\text { E-Operator Training \& } \\
\text { License Examination } \\
\text { F-Adninistrative } \\
\text { G-Operational Error } \\
\text { H-Other }\end{array}$ \\
\hline
\end{tabular}

\begin{tabular}{l} 
METHOD \\
\hline 1-Manual \\
2-Manual Scram \\
3-Auto Scram \\
4-Continued \\
5-Reduced Load \\
9-0ther
\end{tabular}

\section{SYSTEM}

IEEE Standard

$805-1984$ and/or
NUREG-0161 Exhibit F

\section{COMPONENT}

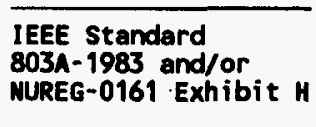

IEEE Standard
$803 A-1983$ and/o

MUREG-0161 Exhibit H

-Administrative

H-Other 


\section{FACILITY DESCRIPTION}

\section{LOCATION}

STATE

COUNTY.

PENnsylvania

DAUPHIK

\section{DIST AND DIRECTION FRON}

MEAREST POPULATION CTR.

TYPE OF REACTOR.

DATE IMITIAL CRITICALITY...... JUNE 05, 1974

DATE IMITIAL ELECTRICITY...... JUNE 19, 1974

date cominercial operate....... September 02, 1974

CONDENSER COOLING METHOD...... COOLING TONERS

CONDENSER COOLING WATER....... SUSQUEHAMNA RIVER

ELECTRIC RELIABILITY

COUNCIL.................. MID-ATLANTIC AREA COUNCIL

\section{UTILITY \& CONTRACTOR INFORMATION}

\section{UTILITY}

LICENSEE

CORPORATE ADDRESS

GPU NUCLEAR CORP.

100 INTERPACE PARKUAY

PARSIPPANY, NEW JERSEY 07054

\section{CONTRACTOR}

ARCHITECT/ENGINEER.

MUC STEAM SYS SUPPLIER

CONSTRUCTOR.

TURBINE SUPPLIER.

REGULATORY INFORMATION

IE REGION RESPONSIBLE.........

IE RESIDENT INSPECTOR......... MICHELLE EVANS

LICENSING PROJ MANAGER......... RONALO W. HERMAN

DOCKET MUMBER............... 50-289

LICENSE \& DATE ISSUANCE........ DPR 050, APRIL 19, 1974 
1. Docket: $50-250$

OPERATING STATUS

2. Reporting Period: DeCEMBER 1994

Outage + On-Line Hrs:

744.0

3. Utility Contact: J.E. KNORR (305) 246-6757

4. Licensed Thermal Power (NWt):

5. Nameplate Rating (Gross MHe):

6. Design Electrical Rating (Net MHe):

7. Maximum Dependable Capacity (Gross MHe):

8. Maximum Dependable Capacity (Net MWe):

9. If Changes Occurred Above Since Last Report, Give Reasons:

10. Power Level To Which Restricted, If Any (Net MHe):

11. Reasons for Restrictions, If Any:

\begin{tabular}{|c|c|c|c|}
\hline & MONTH & YEAR & CUMULATIVE \\
\hline 12. Report Period Hrs & 744.0 & $8,760.0$ & $193,497.6$ \\
\hline 13. Hours Reactor Critical & 717.5 & $7,718.1$ & $128,979.3$ \\
\hline 14. Rx Reserve Shtdwn Hrs & 0.0 & 0.0 & 844.4 \\
\hline 15. Hrs Generator On-Line & 662.0 & $7,515.3$ & $127,049.0$ \\
\hline 16. Unit Reserve Shtdwn Hrs & 0.0 & 0.0 & 121.8 \\
\hline 17. Gross Therm Ener (MLH) & $1,375,950.0$ & $16,026,099.0$ & $264,165,938.4$ \\
\hline 18. Gross Elec Ener (MHH) & $449,048.0$ & $5,179,647.0$ & $84,761,463.0$ \\
\hline 19. Net Elec Ener (MWH) & $425,419.0$ & $4,924,921.0$ & $80,194,426.0$ \\
\hline 20. Unit Service Factor & 89.0 & 85.8 & 65.7 \\
\hline 21. Unit Avail Factor & 89.0 & 85.8 & 65.7 \\
\hline 22. Unit Cap Factor (MDC Net) & 85.9 & 84.4 & 63.2 \\
\hline 23. Unit Cap Factor (DER Net) & 82.5 & 81.1 & 59.8 \\
\hline 24. Unit Forced Outage Rate & 11.0 & 1.9 & 11.3 \\
\hline 25. Forced Outage Hours & 82.0 & 149.4 & $16,169.0$ \\
\hline
\end{tabular}

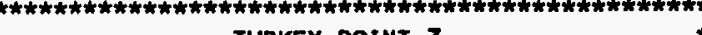 \\ * TURKEY POINT 3 .}

AVERAGE DAILY PONER LEVEL (Net MWe)

\begin{tabular}{ll} 
DAY & PONER \\
\hline & 672 \\
\hline 2 & 681 \\
\hline 3 & 690 \\
\hline 4 & 688 \\
\hline 5 & 684 \\
\hline 6 & 687 \\
\hline 7 & 684 \\
\hline 8 & 683 \\
\hline 9 & 680 \\
\hline 10 & 681 \\
\hline 11 & 684 \\
\hline 12 & 687 \\
\hline 13 & 689 \\
\hline 14 & 692 \\
\hline 15 & 690 \\
\hline
\end{tabular}

\begin{tabular}{cc} 
DAY & PONER \\
\hline 16 & 697 \\
\hline 17 & 622 \\
\hline 18 & 339 \\
\hline 19 & 633 \\
\hline 20 & 693 \\
\hline 21 & 699 \\
\hline 22 & 701 \\
\hline 23 & 672 \\
\hline 24 & 674 \\
\hline 25 & 707 \\
\hline 26 & 30 \\
\hline 27 & 0 \\
\hline 28 & 0 \\
\hline 29 & 48 \\
\hline 30 & 295 \\
\hline 31 & 692 \\
\hline
\end{tabular}

26. Shutdowns Scheduled Over Next Six Months (Type, Date, Duration):

27. If Currently Shutdown, Estimated Startup Date:

Notes:

CUMULATIVE UNIT CAPACITY FACTOR (MDC NET) IS CALCULATED WITH A WEIGHTED AVERAGE. 


\begin{tabular}{|c|c|c|c|c|c|c|c|c|c|}
\hline No. & Date & Type & Hours & Reason & Method & LER Number & System & Component & Cause and Corrective Action To Prevent Recurrence \\
\hline 20 & $12 / 16 / 94$ & $\mathbf{s}$ & 0.0 & B & 5 & & HA & VALVEX & TURBINE VALVE TEST. \\
\hline 21 & $12 / 26 / 94$ & $\mathbf{F}$ & 82.0 & A & 3 & 94006 & нн & INSTRU & AUTOMATIC TRIP CAUSED BY I/P TRANSDUCER FAILURE. \\
\hline
\end{tabular}

\begin{tabular}{|c|c|}
\hline TYPE & REASON \\
\hline $\begin{array}{l}\text { F: Forced } \\
\text { S: Scheduled }\end{array}$ & $\begin{array}{l}\text { A-Equipment Failure } \\
\text { B-Maintenance or Test } \\
\text { C-Refuel ing } \\
\text { D-Regulatory Restriction } \\
\text { E-Operator Training \& } \\
\text { License Examination } \\
\text { F-Administrat ive } \\
\text { G-Operat ional Error } \\
\text { H-Other }\end{array}$ \\
\hline
\end{tabular}

\begin{tabular}{l} 
METHOD \\
\hline 1-Manual \\
2-Manual Scram \\
3-Auto Scram \\
4-Cont inued \\
5-Reduced Load \\
9-0ther
\end{tabular}

SYSTEM

IEEE Standard

NUREG-0161 Exhibit
COMPONENT

IEEE Standard

803A-1983 and/or

MUREG-0161 Exhibit

- Admini strative

H-Other 


\section{FACILITY DESCRIPTION}

\section{LOCATION}

STATE

countr.

FLORIDA

DADE

\section{DIST AMD DIRECTION FROM}

NEAREST POPULATION CTR.....

25 MI S OF MIAMI, FL

TYPE OF REACTOR.

PUR

DATE INITIAL CRITICALITY...... OCTOBER 20, 1972

DATE INITIAL ELECTRICITY....... NOVEMBER 02, 1972

DATE COMMERCIAL OPERATE....... DECEMBER 14, 1972

CONDENSER COOLING METHOD...... CLOSED CAMAL

CONDEMSER COOLING WATER....... CLOSED CYCLE CAMAL

ELECTRIC RELIABILITY

COUNCIL.................... SOUTHEASTERM ELECTRIC RELIABILITY COUNCIL

\section{UTILITY}

LICENSEE..

CORPORATE ADDRESS

FLORIDA POUER \& LIGHT CO.

9250 WEST FLAGLER STREET

MIAMI, FLORIDA 33102

\section{CONTRACTOR}

ARCHITECT/ENGINEER.

BECHTEL

MUC STEAM SYS SUPPLIER...... WESTINGHOUSE

CONSTRUCTOR............... BECHTEL

TURBINE SUPPLIER.......... VESTIMGHOUSE

REGULATORY INFORMATION

IE REGION RESPONSIBLE......... 2

IE RESIDENT INSPECTOR......... THOMAS JOHMSON

LICENSING PROJ MANAGER......... RICHARD P. CROTEAU

DOCKET MUMBER.............. $50-250$

LICENSE \& DATE ISSUANCE........ OPR 031, JULY 19, 1972 


\section{Docket: $50-251$}

OPERATING STATUS

2. Reporting Period: DECEMBER 1994

Outage + On-Line Hrs: $\quad 744.0$

3. Utility Contact: J. E. KNORR (305) 246-6757

4. Licensed Thermal Power (Mut):

5. Nameplate Rating (Gross Mue):

6. Design Electrical Rating (Net MHe):

7. Maximum Dependable Capacity (Gross Mue):

8. Maximum Dependable Capacity (Net Mue):

\begin{tabular}{c}
\hline 2200 \\
\hline 760 \\
\hline 693 \\
\hline 699 \\
\hline 666
\end{tabular}

9. If Changes Occurred Above Since Last Report, Give Reasons:

10. Power Level To thich Restricted, If Any (Net Mue):

11. Reasons for Restrictions, If Any:

\begin{tabular}{|c|c|c|c|}
\hline 12. Report Period Hrs & $\begin{array}{l}\text { MONTH } \\
744.0\end{array}$ & $\begin{array}{l}\text { YEAR } \\
8,760.0 \\
\end{array}$ & $\begin{array}{l}\text { CUMULATIVE } \\
187,229.0\end{array}$ \\
\hline 13. Hours Reactor Critical & 727.1 & $7,567.5$ & $124,848.0$ \\
\hline 14. Rx Reserve Shtdwn Hrs & 0.0 & 0.0 & 166.6 \\
\hline 15. Hrs Generator On-Line & 710.4 & $7,438.6$ & $120,763.5$ \\
\hline 16. Unit Reserve Shtdwn Hrs & 0.0 & $\overline{0.0}$ & 577.3 \\
\hline 17. Gross Therm Ener (MWH) & $1,550,991.0$ & $15,864,754.0$ & $254,924,705.2$ \\
\hline 18. Gross Elec Ener (NWH) & $510,143.0$ & $5,094,680.0$ & $81,448,650.0$ \\
\hline 19. Net Elec Ener (MWH) & $485,850.0$ & $4,844,354.0$ & $77,079,586.0$ \\
\hline 20. Unit Service Factor & 95.5 & 84.9 & 64.5 \\
\hline 21. Unit Avail Factor & 95.5 & 84.9 & 64.8 \\
\hline 22. Unit Cap Factor (MDC Net) & 98.1 & 83.0 & 62.8 \\
\hline 23. Unit Cap Factor (DER Net) & 94.2 & 79.8 & 59.4 \\
\hline 24. Unit Forced Outage Rate & 4.5 & 3.9 & 11.0 \\
\hline 25. Forced Outage Hours & 33.6 & 298.6 & $14,906.7$ \\
\hline
\end{tabular}

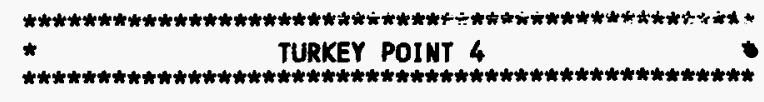

AVERAGE DAILY POWER LEVEL (Net MWe)

\begin{tabular}{|c|c|c|c|}
\hline DAY & PONER & DAY & PONER \\
\hline 1 & 0 & 16 & 700 \\
\hline 2 & 199 & 17 & 697 \\
\hline 3 & 688 & 18 & 694 \\
\hline 4 & 688 & 19 & 688 \\
\hline 5 & 685 & 20 & 694 \\
\hline 6 & 687 & 21 & 704 \\
\hline 7 & 689 & 22 & 696 \\
\hline 8 & 684 & 23 & 673 \\
\hline 9 & 684 & 24 & 675 \\
\hline 10 & 685 & 25 & 675 \\
\hline 11 & 688 & 26 & 707 \\
\hline 12 & 690 & 27 & 704 \\
\hline 13 & 692 & 28 & 709 \\
\hline 14 & 696 & 29 & 700 \\
\hline \multirow[t]{2}{*}{15} & 698 & 30 & 700 \\
\hline & & 31 & 696 \\
\hline
\end{tabular}

26. Shutdowns Scheduled Over Next Six Months (Type, Date, Duration):

27. If Currently Shutdown, Estimated Startup Date:

Notes:

CUMULATIVE UNIT CAPACITY FACTOR (MOC NET) IS CALCULATED WITH A WEIGHTED AVERAGE. 


\begin{tabular}{cccccccccc}
\hline No. & Date & Type & Hours & Reason & Method & LER Number & System & Component & Cause and Corrective Action To Prevent Recurrence \\
\hline 13 & $11 / 30 / 94$ & F & 33.6 & A & 4 & 94006 & EB & ELECON & REACTOR TRIP DUE TO TURBINE GEMERATOR GROUND.
\end{tabular}

$11 / 30 / 94$

A

94006

REACTOR TRIP DUE TO TURBINE GEMERATOR GROUND.

\begin{tabular}{ll} 
TYPE & REASON \\
\hline F: Forced & $\begin{array}{l}\text { A-Equipment Failure } \\
\text { B-Maintenance or Test } \\
\text { C-Refuel ing } \\
\text { D-Regulatory Restriction } \\
\text { E-Operator Iraining \& } \\
\text { License Examination } \\
\text { F-Administrative } \\
\text { G-Operational Error } \\
\text { H-Other }\end{array}$
\end{tabular}

\begin{tabular}{l} 
METHOD \\
\hline 1-Manual Scram \\
2-Manual Scram \\
3-Auto Scram \\
4-Cont inued \\
5-Reduced Load \\
9-0ther
\end{tabular}

\section{SYSTEM}

IEEE Standard

805-1984 and/or

MUREG-0161 Exhibit $F$

\section{COMPONENT}

IEEE Standard

803A-1983 and/or
NUREG-0161 Exhibit H

F-Adninistrative

H-other 


\section{FACILITY DESCRIPTION}

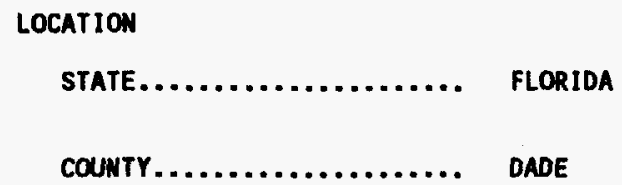

\section{UTILITY \& CONTRACTOR INFORMATION}

UTILITY

LICENSEE................. FLORIDA POUER \& LIGHT CO.

CORPORATE ADDRESS......... 9250 UEST FLAGLER STREET MIAMI, FLORIDA 33102

\section{CONTRACTOR}

ARCHI TECT/ENGIMEER.......... BECHTEL

MUC STEAM SYS SUPPLIER...... WESTIMGHOUSE

CONSTRUCTOR............... BECHTEL

TURBINE SUPPLIER........... WESTIMGHOUSE

REGULATORY INFORMATION

IE REGLON RESPONSIBLE......... 2

IE RESIDENT INSPECTOR......... THOMAS JOHMSON

LICENSING PROJ MANAGER......... RICHARD P. CROTEAU

DOCKET NUMBER............... 50-251

LICENSE \& DATE ISSUANCE........ DPR 041, APRIL 10, 1973 
1. Docket: $50-271$

OPERATING STATUS

2. Reporting Period: DECEMBER 1994

Outage + On-Line Hrs:

744.0

3. Utility Contact: G. A. WALLIN (802) 257-7711

4. Licensed Thermal Power (MUt):

5. Nameplate Rating (Gross MWe):

6. Design Electrical Rating (Net MWe):

7. Maximm Dependable Capacity (Gross MHe):

8. Maximum Dependable Capacity (Net MWe):

9. If Changes Occurred Above Since Last Report, Give Reasons:

10. Power Level To Which Restricted, If Any (Net MHe):

11. Reasons For Restrictions, If Any:

\begin{tabular}{|c|c|c|c|}
\hline & MONTH & YEAR & CUMULATIVE \\
\hline 12. Report Period Hrs & 744.0 & $8,760.0$ & $193,584.0$ \\
\hline 13. Hours Reactor Critical & 744.0 & $8,646.2$ & $159,863.6$ \\
\hline 14. Rx Reserve Shtdwn Hrs & 0.0 & 0.0 & 0.0 \\
\hline 15. Hrs Generator On-Line & 744.0 & $8,601.4$ & $156,717.1$ \\
\hline 16. Unit Reserve Shtdun Hrs & 0.0 & 0.0 & 0.0 \\
\hline 17. Gross Therm Ener (MWH) & $1,181,396.2$ & $13,464,204.5$ & $234,169,876.0$ \\
\hline 18. Gross Elec Ener (MUH) & $400,509.0$ & $4,517,970.0$ & $78,037,352.0$ \\
\hline 19. Net Elec Ener (MWH) & $384,825.0$ & $4,315,550.0$ & $74,162,574.0$ \\
\hline 20. Unit Service Factor & 100.0 & 98.2 & 81.0 \\
\hline 21. Unit Avail Factor & 100.0 & 98.2 & 81.0 \\
\hline 22. Unit Cap Factor (MDC Net) & 102.6 & 97.7 & 76.0 \\
\hline 23. Unit Cap Factor (DER Net) & 100.6 & 95.8 & 74.5 \\
\hline 24. Unit forced Outage Rate & 0.0 & 1.2 & 5.2 \\
\hline 25. Forced Outage Hours & 0.0 & 104.6 & $8,622.3$ \\
\hline
\end{tabular}

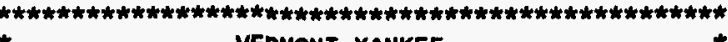 \\ * VERMONT YANKEE}

AVERAGE DAILY POWER LEVEL (Net MUe)

\begin{tabular}{lc} 
DAY & PONER \\
\hline 1 & 518 \\
\hline 2 & 486 \\
\hline 3 & 519 \\
\hline 4 & 518 \\
\hline 5 & 519 \\
\hline 6 & 517 \\
\hline 7 & 518 \\
\hline 8 & 518 \\
\hline 9 & 518 \\
\hline 10 & 518 \\
\hline 11 & 517 \\
\hline 12 & 518 \\
\hline 13 & 518 \\
\hline 14 & 518 \\
\hline 15 & 518 \\
\hline
\end{tabular}

\begin{tabular}{ll} 
DAY & PONER \\
\hline 16 & 519 \\
\hline 17 & 519 \\
\hline 18 & 516 \\
\hline 19 & 519 \\
\hline 20 & 521 \\
\hline 21 & 519 \\
\hline 22 & 519 \\
\hline 23 & 519 \\
\hline 24 & 519 \\
\hline 25 & 517 \\
\hline 26 & 519 \\
\hline 27 & 519 \\
\hline 28 & 519 \\
\hline 29 & 519 \\
\hline 30 & 515 \\
\hline 31 & 519 \\
\hline
\end{tabular}

26. Shutdowns Scheduled Over Next Six Months (Type, Date, Duration): REFUELING OUTAGE, MARCH 18, 1995, SIX WEEKS.

27. If Currently Shutdown, Estimated Startup Date: Notes:

CUMULATIVE VALUES FOR ITEMS 13, 15, AND 17-19 INCLUDE PRECOMMERCIAL DATA, WHILE CUMULATIVE VALUES FOR ITEMS 20-25 ARE CALCULATED SINCE COMNERCIAL OPERATION. 


\begin{tabular}{|c|c|c|c|c|c|c|c|c|c|}
\hline No. & Date & Type & Hours & Reason & Method & LER Number & System & Component & Cause and Corrective Action To Prevent Recurrence \\
\hline $94-14$ & $12 / 02 / 94$ & $\mathbf{s}$ & 0.0 & B & 5 & & RB & CONROD & MSIV CLOSURE AND BYPASS VALVE TESTS, AND A ROD \\
\hline
\end{tabular}

$\mathbf{s}$

B PATTERN EXCHANGE.

\begin{tabular}{l} 
TYPE \\
\hline F: Forced \\
S: Scheduled
\end{tabular}

\section{REASON}

A-Equipment Failure

B-Maintenance or Test

C-Refuel ing

D-Regulatory Restriction

E-Operator Training \&

License Examination

F-Administrative

G-Operational Error

H-Other

\section{METHOD}

1-Manual

2-Manual Scram

3-Auto Scram

4-Cont inued

S-Recuced Load

9-0ther

\begin{tabular}{l} 
SYSTEM \\
\hline IEEE Standard \\
$805-1984$ and/or \\
MUREG-0161 Exhibit
\end{tabular}

MUREG-0161 Exhibit $F$

\section{COMPONENT}

IEEE Standard

803A-1983 and/or

MUREG-0161 Exhibit $H$ 


\section{FACILITY DESCRIPTION}

\section{LOCATION}

STATE...........

COUNTY

VERMONT

WINDHAM

DIST AND DIRECTION FROH

MEAREST POPULATION CTR..... 5 MI S OF BRATTLEBORO, VT

TYPE OF REACTOR

DATE INITIAL CRITICALITY...... MARCH 24, 1972

DATE INITIAL ELECTRICITY...... SEPTEMBER 20, 1972

DATE COMMERCIAL OPERATE....... MOVEMBER 30,1972

CONDENSER COOLING METHOD...... COOLING TONER

COMDENSER COOLING WATER.

CONNECTICUT RIVER

ELECTRIC RELIABILITY

COUNCIL.................... MORTHEASTERN POWER COORDINATION COUNCIL
UTILITY \& CONTRACTOR INFORMATION

\section{UTILITY}

LICENSEE.

VERMONT YANKEE MUCLEAR PONER CORP.

CORPORATE ACDRESS.

\section{RD \#5, BOX 169 FERRY ROAD}

BRATTLEBORO, VÉRMONT 05301

\author{
CONTRACTOR \\ ARCHITECT/ENGINEER......... EBASCO \\ NUC STEAM SYS SUPPLIER...... GENERAL ELECTRIC \\ CONSTRUCTOR............... EBASCO \\ TURBINE SUPPLIER........... GENERAL ELECTRIC
}

\section{REGULATORY INFORMATION}

IE REGION RESPONSIBLE.........

IE RESIDENT INSPECTOR.

HAROLD EICHENHOLZ

LICENSING PROJ MANAGER

DANIEL H. DORMAN

DOCKET NUMBER

$50-271$

LICENSE \& DATE ISSUANCE

DPR 028, FEBRUARY 28, 1973 
1. Docket: $50-424$

OPERATING STATUS

2. Reporting Period: DECEMBER 1994

Outage + On-Line Hrs:

744.0

3. Utility Contact: G. L. HOOPER (706) 826-4104

4. Licensed Thermal Power (MUt):

5. Nameplate Rating (Gross Mue):

6. Design Electrical Rating (Net Mue):

7. Maximum Dependable Capacity (Gross MUe):

8. Maximum Dependable Capacity (Net MWe):

\begin{tabular}{c}
3565 \\
\hline 1215 \\
\hline 1169 \\
\hline 1223 \\
\hline 1169
\end{tabular}

9. If Changes Occurred Above Since Last Report, Give Reasons:

10. Power Level To Which Restricted, If Any (Net MHe):

11. Reasons For Restrictions, If Any:

\begin{tabular}{|c|c|c|c|}
\hline & MONTH & YEAR & CUMULATIVE \\
\hline 12. Report Period Hrs & 744.0 & $8,760.0$ & $66,505.0$ \\
\hline 13. Hours Reactor Critical & 744.0 & $7,889.5$ & $57,764.7$ \\
\hline 14. Rx Reserve Shtdun Hrs & 0.0 & 0.0 & 0.0 \\
\hline 15. Hrs Generator On-Line & 744.0 & $7,848.0$ & $56,720.9$ \\
\hline 16. Unit Reserve Shtdwn Hrs & 0.0 & 0.0 & $\overline{0.0}$ \\
\hline 17. Gross Therm Ener (ММH) & $2,631,652.0$ & $27,072,103.0$ & $190,793,301.0$ \\
\hline 18. Gross Elec Ener (MUK) & $905,180.0$ & $9,243,725.0$ & $64,226,687.0$ \\
\hline 19. Net Elec Ener (MUH) & $866,060.0$ & $8,817,155.0$ & $61,058,447.0$ \\
\hline 20. Unit Service Factor & 100.0 & 89.6 & 85.3 \\
\hline 21. Unit Avail Factor & 100.0 & 89.6 & 85.3 \\
\hline 22. Unit Cap Factor (MDC Net) & 99.6 & 86.1 & 83.0 \\
\hline 23. Unit Cap Factor (DER Net) & 99.6 & 86.1 & 82.3 \\
\hline 24. Unit forced Outage Rate & 0.0 & 0.6 & 4.9 \\
\hline 25. Forced Outage Hours & 0.0 & 49.0 & $2,913.2$ \\
\hline
\end{tabular}

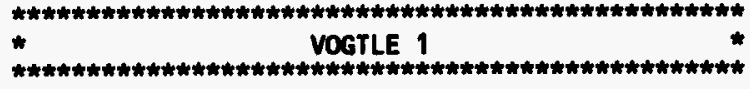

AVERAGE DAILY PONER LEVEL (Het me)

\begin{tabular}{ll} 
DAY & POUER \\
\hline 1 & 1179 \\
\hline 2 & 1176 \\
\hline 3 & 1171 \\
\hline 4 & 1164 \\
\hline 5 & 1166 \\
\hline 6 & 1171 \\
\hline 7 & 1172 \\
\hline 8 & 1173 \\
\hline 9 & 1175 \\
\hline 10 & 1172 \\
\hline 11 & 1178 \\
\hline 12 & 1180 \\
\hline 13 & 1180 \\
\hline 14 & 1179 \\
\hline 15 & 1177 \\
\hline
\end{tabular}

\begin{tabular}{cc} 
DAY & POUER \\
\hline 16 & 1178 \\
\hline 17 & 1179 \\
\hline 18 & 1176 \\
\hline 19 & 1182 \\
\hline 20 & 1181 \\
\hline 21 & 1178 \\
\hline 22 & 1177 \\
\hline 23 & 1175 \\
\hline 24 & 1176 \\
\hline 25 & 1180 \\
\hline 26 & 1097 \\
\hline 27 & 1058 \\
\hline 28 & 1056 \\
\hline 29 & 1121 \\
\hline 30 & 1178 \\
\hline 31 & 1181 \\
\hline
\end{tabular}

26. Shutdowns Scheduled Over Mext Six Months (Type, Date, Duration):

27. If Currently Shutdown, Estimated Startup Date:

Notes:

YEAR-TO-DATE AND CUMULATIVE UNIT CAPACITY FACTORS (MDC \& DER) ARE CALCULATED WITH . WEIGHTED AVERAGES. 


\begin{tabular}{|c|c|c|c|c|}
\hline TYPE & REASON & METHOD & SYSTEM & COMPONENT \\
\hline $\begin{array}{l}\text { F: Forced } \\
\text { S: Scheduled }\end{array}$ & $\begin{array}{l}\text { A-Equipment Failure } \\
\text { B-Maintenance or Test } \\
\text { C-Refuel ing } \\
\text { D-Regulatory Restriction } \\
\text { E-Operator Training \& } \\
\text { License Examination } \\
\text { F-Administrative } \\
\text { G-Operational Error } \\
\text { H-Other }\end{array}$ & $\begin{array}{l}\text { 1-Manual } \\
\text { 2-Manual Scram } \\
\text { 3-Auto Scram } \\
\text { 4-Cont inued } \\
\text { 5-Reduced Load } \\
\text { 9-0ther }\end{array}$ & $\begin{array}{l}\text { IEEE Standard } \\
805-1984 \text { and/or } \\
\text { NUREG-0161 Exhibit F }\end{array}$ & $\begin{array}{l}\text { IEEE standard } \\
803 \mathrm{~A}-1983 \text { and/or } \\
\text { NUREG-0161 Exhibit H }\end{array}$ \\
\hline
\end{tabular}




\section{FACILITY DESCRIPTION}

LOCATION

STATE.

COUNTY.

DIST AND DIRECTION FROM

NEAREST POPULATION CTR.

TYPE OF REACTOR

DATE INITIAL CRITICALITY...... MARCH 09, 1987

DATE INITIAL ELECTRICITY...... MARCH 27, 1987

DATE COMMERCIAL OPERATE........ JUME 01, 1987

CONDENSER COOLING METHOD...... CCCT

CONDENSER COOLING MATER....... SAVANMAH RIVER

ELECTRIC RELIABILITY

COUNCIL................. SOUTHEASTERN ELECTRIC RELIABILITY COUNCIL

\section{UTILITY \& CONTRACTOR INFORMATION}

UTILITY

LICENSEE.

CORPORATE ADDRESS

\section{CONTRACTOR}

ARCHITECT/ENGINEER

MUC STEAM SYS SUPPLIER...... MESTIMGHOUSE

CONSTRUCTOR

GEORGIA PONER CO.

TURBINE SUPPLIER.

GENERAL ELECTRIC

\section{REGULATORY INFORMATION}

IE REGION RESPONSIBLE........ 2

IE RESIDENT INSPECTOR.......... BRIAN R. BONSER

LICENSING PROJ MANAGER.......... DARL S. HOOD

DOCKET MUMBER.............. 50-424

LICENSE \& dATE ISSUANCE........ NPF 068, MARCH 16, 1987 


\section{Docket: $50-425$}

OPERATING STATUS

2. Reporting Period: DECEMBER 1994

Outage + On-Line Hrs:

744.0

3. Utility Contact: G. L. HOOPER (706) 826-4104

4. Licensed Thermal Power (MWt):

5. Naneplate Rating (Gross MWe):

6. Design Electrical Rating (Net MWe):

7. Maximum Dependable Capacity (Gross MWe):

8. Maximm Dependable Capacity (Net MHe):

\begin{tabular}{c}
3565 \\
\hline 1215 \\
\hline 1169 \\
\hline 1223 \\
\hline 1169
\end{tabular}

9. If Changes Occurred Above Since Last Report, Give Reasons:

10. Power Level To Which Restricted, If Any (Net Mue):

11. Reasons for Restrictions, If Any:

\begin{tabular}{|c|c|c|c|}
\hline & MONTH & YEAR & CUMULATIVE \\
\hline 12. Report Period Hrs & 744.0 & $8,760.0$ & 49.249 .0 \\
\hline 13. Hours Reactor Critical & 744.0 & $8,107.4$ & $44,147.9$ \\
\hline 14. Rx Reserve Shtdun Hrs & 0.0 & 0.0 & 0.0 \\
\hline 15. Hrs Generator On-Line & 744.0 & $8,063.8$ & $43,590.4$ \\
\hline 16. Unit Reserve Shtdwn Hrs & 0.0 & 0.0 & 0.0 \\
\hline 17. Gross Therm Ener (МUH) & $2,649,055.0$ & $28,479,130.0$ & $145,558,628.0$ \\
\hline 18. Gross Elec Ener (MHH) & $916,522.0$ & $9,766,659.0$ & $49,448,696.0$ \\
\hline 19. Net Elec Ener (MUH) & $878,362.0$ & $9,331,599.0$ & $47,079,076.0$ \\
\hline 20. Unit Service Factor & 100.0 & 92.1 & 88.5 \\
\hline 21. Unit Avail Factor & 100.0 & 92.1 & 88.5 \\
\hline 22. Unit Cap Factor (MDC Net) & 101.0 & 91.2 & 85.4 \\
\hline 23. Unit Cap Factor (DER Net) & $101=0$ & 91.1 & 85.4 \\
\hline 24. Unit Forced Outage Rate & 0.0 & 4.9 & 2.2 \\
\hline 25. Forced Outage Hours & 0.0 & 411.3 & 969.2 \\
\hline
\end{tabular}

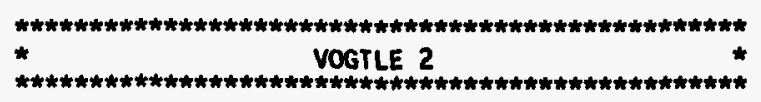

AVERAGE DAILY POWER LEVEL (Net MUe)

\begin{tabular}{ll} 
DAY & PONER \\
\hline 1 & 1183 \\
\hline 2 & 1174 \\
\hline 3 & 1167 \\
\hline 4 & 1161 \\
\hline 5 & 1165 \\
\hline 6 & 1170 \\
\hline 7 & 1175 \\
\hline 8 & 1179 \\
\hline 9 & 1183 \\
\hline 10 & 1180 \\
\hline 11 & 1184 \\
\hline 12 & 1187 \\
\hline 13 & 1187 \\
\hline 14 & 1186 \\
\hline 15 & 1183 \\
\hline
\end{tabular}

\begin{tabular}{cl} 
DAY & PONER \\
\hline 16 & 1184 \\
\hline 17 & 1184 \\
\hline 18 & 1182 \\
\hline 19 & 1184 \\
\hline 20 & 1184 \\
\hline 21 & 1185 \\
\hline 22 & 1184 \\
\hline 23 & 1183 \\
\hline 24 & 1184 \\
\hline 25 & 1185 \\
\hline 26 & 1183 \\
\hline 27 & 1184 \\
\hline 28 & 1182 \\
\hline 29 & 1182 \\
\hline 30 & 1184 \\
\hline 31 & 1183 \\
\hline
\end{tabular}

26. Shutdowns Scheduled Over Mext Six Months (Type, Date, Duration): REFUELING OUTAGE, FEBRUARY 25, 1995, 30 DAYS.

27. If Currently Shutdown, Estimated Startup Date:

Hotes:

YEAR-TO-DATE AND CUMULATIVE UNIT CAPACITY FACTORS (MDC \& DER) ARE CALCULATED WITH MEIGHTED AVERAGES. 


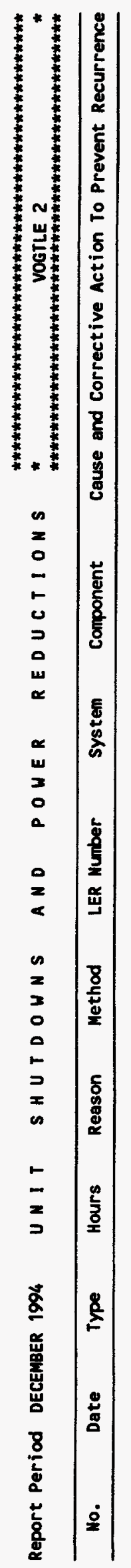

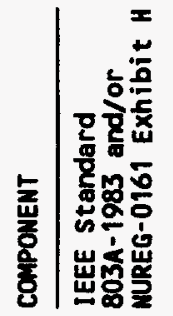

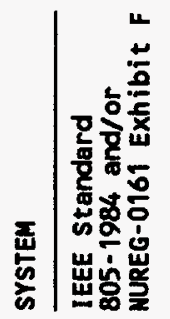

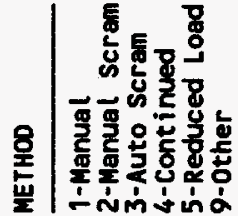

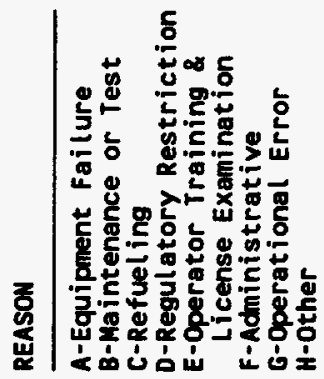

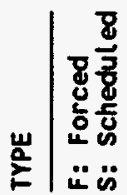




\section{FACILITY DESCRIPTION}

\section{LOCATION}

$$
\begin{aligned}
& \text { STATE.................. GEORGIA } \\
& \text { COUNTY................ BURKE }
\end{aligned}
$$

\section{DIST AMD DIRECTION FROM}

MEAREST POPULATION CTR..... 26 MI SE OF AUGUSTA, GA

TYPE OF REACTOR.

PUR

DATE INITIAL CRITICALITY...... MARCH 28, 1989

DATE INITIAL ELECTRICITY...... APRIL 10, 1989

DATE COMMERCIAL OPERATE...... MAY 20, 1989

CONDEMSER COOLING METHOD...... CCCT

CONDENSER COOLING HATER....... SAVAMNAH RIVER

ELECTRIC RELIABILITY

COUNCIL.................. S SOUTHEASTERN ELECTRIC

\section{UTILITY \& CONTRACTOR INFORMATION}

UTILITY

LICENSEE................... GEORGIA POWER CO.

CORPORATE ADDRESS.

P.O. BOX 1295

BirMinghaM, ALABAMA 35201

\section{CONTRACTOR}

ARCHITECT/ENGINEER.......... SOUTHERN SERVICES \& BECHTEL

NUC STEAM SYS SUPPLIER...... MESTINGHOUSE

CONSTRUCTOR.............. GEORGIA PONER CO.

TURBINE SUPPLIER.......... GENERAL ELECTRIC

\section{REGULATORY INFORMATION}

IE REGION RESPONSIBLE.........

IE RESIDENT INSPECTOR

BRIAN R. BONSER

LICENSING PROJ MANAGER

DARL S. HOOO

DOCKET NUMBER

$50-425$

LICENSE \& DATE ISSUANCE 
1. Docket: $50-397$

OPERATING STATUS

2. Reporting Period: DECEMBER 1994 outage + On-Line Hrs: $\mathbf{7 4 4 . 0}$

3. Utility Contact: DAVID G. EMBREE (509) $377-8448$

4. Licensed Thermal Power (MHT):

5. Nameplate Rating (Gross MHe):

6. Design Electrical Rating (Net MWe):

7. Maximm Dependable Capacity (Gross MMe):

8. Maximum Dependable Capacity (Net MHe):

9. If Changes Occurred Above Since Last Report, Give Reasons:

10. Power Level To Which Restricted, If Any (Net MWe):

11. Reasons for Restrictions, If Any:

\begin{tabular}{|c|c|c|c|}
\hline 12. Report Period Hrs & $\begin{array}{l}\text { MONTH } \\
744.0\end{array}$ & $\begin{array}{l}\text { YEAR } \\
8,760.0\end{array}$ & $\begin{array}{l}\text { CUMULATIVE } \\
88,088.0\end{array}$ \\
\hline 13. Hours Reactor Critical & 744.0 & $6,590.4$ & $62,700.6$ \\
\hline 14. Rx Reserve Shtdwn Hrs & 0.0 & $\overline{0.0}$ & 340.4 \\
\hline 15. Hrs Generator On-Line & 744.0 & $6,500.6$ & $60,567.8$ \\
\hline 16. Unit Reserve Shtdun Mrs & 0.0 & 0.0 & 381.7 \\
\hline 17. Gross Therm Ener (MUH) & $2,463,811.0$ & $20,501,534.0$ & $179,262,840.0$ \\
\hline 18. Gross Elec Ener (NWH) & $857,780.0$ & $7,041,290.0$ & $60,353,500.0$ \\
\hline 19. Net Elec Ener (MWH) & $826,508.0$ & $6,739,749.0$ & $57,825,132.0$ \\
\hline 20. Unit Service Factor & 100.0 & $\overline{74.2}$ & 68.8 \\
\hline 21. Unit Avail Factor & 100.0 & 74.2 & 69.2 \\
\hline 22. Unit Cap Factor (MDC Net) & 102.3 & 70.8 & 60.1 \\
\hline 23. Unit Cap Factor (DER Net) & 99.2 & 68.7 & 59.5 \\
\hline 24. Unit Forced Outage Rate & 0.0 & 1.3 & 11.6 \\
\hline 25. Forced Outage Hours & 0.0 & 85.8 & $7,971.1$ \\
\hline
\end{tabular}

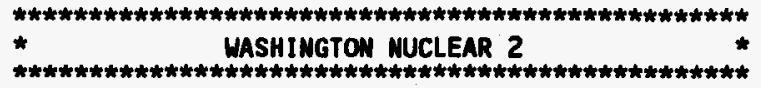

AVERAGe DAILY PONER LeVEL (Net MUe)

\begin{tabular}{ll} 
DAY & PONER \\
\hline 1 & 1106 \\
\hline 2 & 1108 \\
\hline 3 & 1120 \\
\hline 4 & 1118 \\
\hline 5 & 1117 \\
\hline 6 & 1119 \\
\hline 7 & 1119 \\
\hline 8 & 1114 \\
\hline 9 & 1114 \\
\hline 10 & 1118 \\
\hline 11 & 1118 \\
\hline 12 & 1114 \\
\hline 13 & 1115 \\
\hline 14 & 1117 \\
\hline 15 & 1116 \\
\hline
\end{tabular}

\begin{tabular}{cc} 
DAY & PONER \\
\hline 16 & 1111 \\
\hline 17 & 1032 \\
\hline 18 & 1107 \\
\hline 19 & 1105 \\
\hline 20 & 1089 \\
\hline 21 & 1106 \\
\hline 22 & 1118 \\
\hline 23 & 1116 \\
\hline 24 & 1117 \\
\hline 25 & 1119 \\
\hline 26 & 1110 \\
\hline 27 & 1112 \\
\hline 28 & 1109 \\
\hline 29 & 1121 \\
\hline 30 & 1118 \\
\hline 31 & 1119 \\
\hline
\end{tabular}

26. Shutdowns Scheduled Over Hext Six Months (Type, Date, Duration): REFUELING OUTAGE, APRIL 15, 1995, 42 DAYS.

27. If Currently Shutdown, Est imated Startup Date:

Notes:

CUMULATIVE UNIT CAPACITY FACTORS (MDC \& DER) ARE CALCULATED WITH WEIGHTED AVERAGES. 


No. Date Type Hours Reason Method LER Number System Component Cause and Corrective Action To Prevent Recurrence

\section{TYPE}

F: Forced

S: Scheduled

\section{REASON}

A-Equipment Failure

B-Maintenance or Test

C-Refuel ing

D-Regulatory Restriction

E-Operator Training \&

License Examination

r-Administrative

G-Operational Error

H-othe

\section{SYSTEM}

METHOD

1-Manual

2-Manual Scran

3-Auto Scram

4-Cont inued

9-Reduced Load

9-other
IEEE Standard

NUREG-0161 Exhibit F
COMPONENT

IEEE Standard

MUREG-0161 Exhibit H 


\section{FACILITY DESCRIPTION}

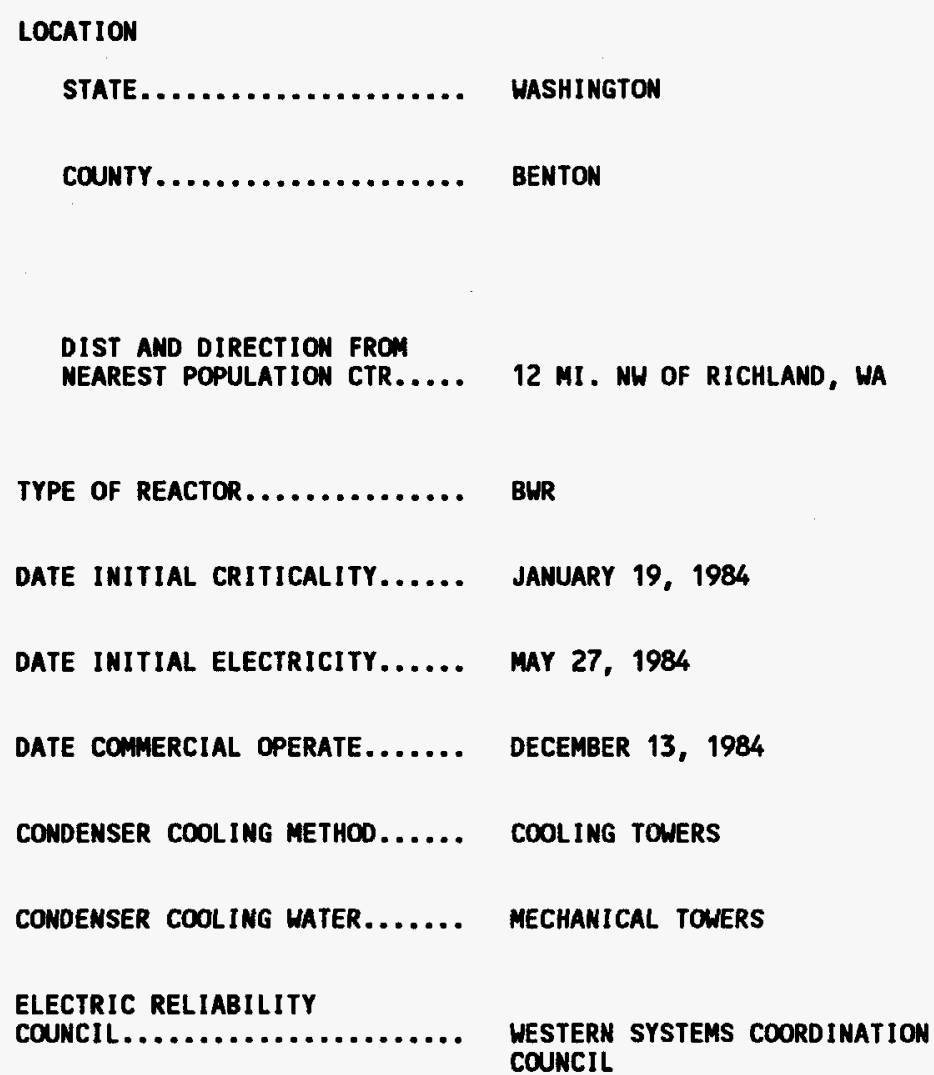

\section{UTILITY \& CONTRACTOR INFORMATION}

UTILITY

LICENSEE................ WASHINGTON PUBLIC PONER SUPPLY SYSTEM

CORPORATE ADDRESS

P.O. BOX 968
RICHLAND, WASHINGTON 99352

\section{CONTRACTOR}

ARCHITECT/ENGINEER......... BURNS \& ROE

NUC STEAM SYS SUPPLIER...... GENERAL ELECTRIC

CONSTRUCTOR............... BECHTEL

TURBINE SUPPLIER.......... WESTINGHOUSE

\section{REGULATORY INFORMATION}

IE REGION RESPONSIBLE........ 4

IE RESIDENT INSPECTOR .

ROBERT BARR

LICENSING PROJ MANAGER

JAMES W. CLIFFORD

DOCKET MUMBER

$50-397$

LICENSE \& DATE ISSUANCE. 
1. Docket: $50-382$

OPERATING STATUS

2. Reporting Period: DECEMBER 1994

Outage + On-Line Hrs:

744.0

3. Utility Contact: T. S. BECKER (504) 739-6683

4. Licensed Thermal Power (MWt):

5. Nameplate Rating (Gross MWe):

6. Design Electrical Rating (Wet MWe):

7. Maximm Dependable Capacity (Gross MWe):

8. Maximum Dependable Capacity (Net MWe):

9. If Changes Occurred Above Since Last Report, Give Reasons:

10. Power Level To Which Restricted, If Any (Net MHe):

11. Reasons For Restrictions, If Any:

MONTH YEAR CUMULATIVE

12. Report Period Hrs

13. Hours Reactor Critical

14. Rx Reserve Shtdun Hrs

15. Hrs Generator On-Line

16. Unit Reserve Shtdwn Hrs

17. Gross Therm Ener (MUH)

18. Gross Elec Ener (MUH)

19. Net Elec Ener (MUH)

20. Unit Service Factor

21. Unit Avail Factor

22. Unit Cap Factor (MDC Net)

23. Unit Cap Factor (DER Net)

24. Unit Forced Outage Rate

25. Forced Outage Hours

\section{MONTH}

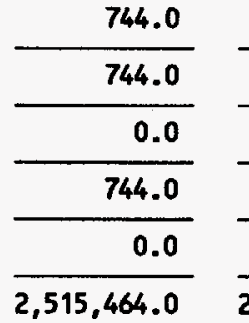

$835,620.0$

$\begin{array}{r}801,685.0 \\ \hline 100.0\end{array}$

$\begin{array}{r}100.0 \\ 100.0 \\ \hline 100.2\end{array}$

100.2

97.6

$\begin{array}{r}0.0 \\ \hline 0.0\end{array}$

\begin{tabular}{|c|c|}
\hline $8,760.0$ & $81,265.0$ \\
\hline 7.622 .8 & $68,722.8$ \\
\hline 0.0 & 0.0 \\
\hline $7,556.1$ & $67,844.3$ \\
\hline 0.0 & 0.0 \\
\hline $25,301,132.0$ & $224,329,609.0$ \\
\hline $8,313,680.0$ & $74,734,460.0$ \\
\hline $7,931,921.0$ & $71,242,364.0$ \\
\hline 86.3 & 83.5 \\
\hline 86.3 & 83.5 \\
\hline 84.2 & 81.6 \\
\hline 82.0 & 79.4 \\
\hline 0.4 & 3.2 \\
\hline 31.5 & $2,210.0$ \\
\hline
\end{tabular}

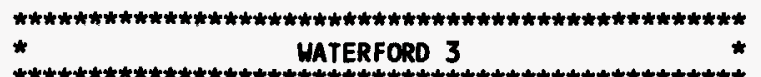

* MATERFORD 3 i

AVERAGE DAILY POWER LEVEL (Net MWe)

\begin{tabular}{ll} 
DAY & POWER \\
\hline 1 & 1075 \\
\hline 3 & 1077 \\
\hline 4 & 1001 \\
\hline 5 & 1077 \\
\hline 6 & 1077 \\
\hline 7 & 1077 \\
\hline 8 & 1078 \\
\hline 9 & 1078 \\
\hline 10 & 1079 \\
\hline 11 & 1079 \\
\hline 12 & 1079 \\
\hline 13 & 1077 \\
\hline 14 & 1079 \\
\hline 15 & 1081 \\
\hline
\end{tabular}

\begin{tabular}{cc} 
DAY & PONER \\
\hline 16 & 1080 \\
\hline 17 & 1081 \\
\hline 18 & 1081 \\
\hline 19 & 1082 \\
\hline 20 & 1082 \\
\hline 21 & 1083 \\
\hline 22 & 1083 \\
\hline 23 & 1082 \\
\hline 24 & 1083 \\
\hline 25 & 1083 \\
\hline 26 & 1082 \\
\hline 27 & 1080 \\
\hline 28 & 1081 \\
\hline 29 & 1083 \\
\hline 30 & 1082 \\
\hline 31 & 1083 \\
\hline
\end{tabular}

26. Shutdowns Scheduled Over Next Six Months (Type, Date, Duration):

27. If Currently Shutdown, Estimated Startup Date:

Motes: 


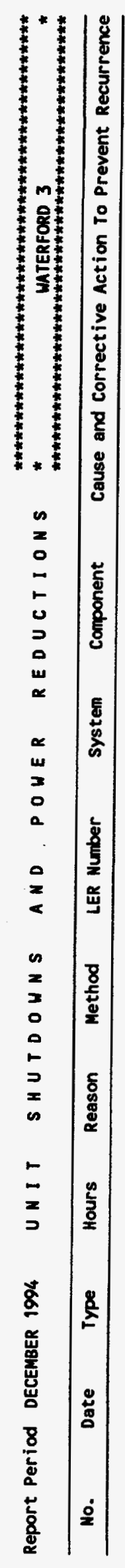

$n$
$\dot{n}$
$\dot{0}$
$\dot{8}$
0

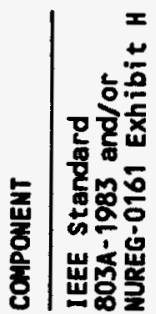

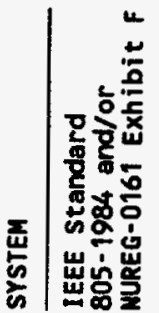

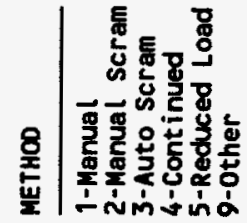

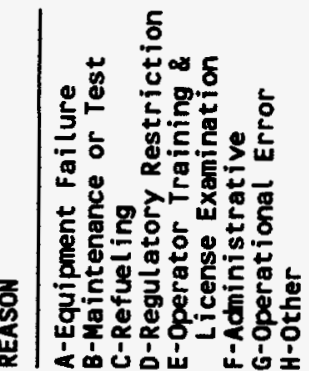

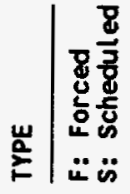




\section{FACILITY DESCRIPTION}

\section{LOCATION}

STATE

COUNTY.

\section{DIST AND DIRECTION FROM} NEAREST POPULATION CTR.....

TYPE OF REACTOR.

DATE INITIAL CRITICALITY.

DATE INITIAL ELECTRICITY......

DATE COMNERCIAL OPERATE.

CONDENSER COOLING METHOD

CONDENSER COOLING WATER.

ELECTRIC RELIABILITY

COUNCIL.
LOUISIANA

ST CHARLES

20 MI $\mathrm{H}$ OF MEH ORLEANS, LA

PUR

MARCH 04, 1985

MARCH 18, 1985

SEPTEMBER 24, 1985

ONCE THRU

MISSISSIPPI RIVER

SOUTHWEST POWER POOL

\section{UTILITY \& CONTRACTOR INFORMATION}

UTILITY

LICENSEE.

CORPORATE ADDRESS

CONTRACTOR

ARCHITECT/ENGINEER

MUC STEAM SYS SUPPLIER...... COMBUSTION ENGINEERING

CONSTRUCTOR.

TURBINE SUPPLIER

EBASCO

MESTINGHOUSE

REGULATORY INFORMATION

IE REGION RESPONSIBLE.........

IE RESIDENT INSPECTOR......... EDWARD FORD

LICENSING PROJ MANAGER.......... DAVID L. HIGGINTON

DOCKET MUMBER............. 50-382

LICENSE \& DATE ISSUANCE......... NPF 038, MARCH 16, 1985 
1. Docket: $50-482$

OPERATING STATUS

2. Reporting Period: DECEMBER 1994

Outage + On-Line Hrs: $\quad 744.0$

3. Utility Contact: M. MILLIAMS (316) 364-8831

4. Licensed Thermal Power (MWt):

5. Nameplate Rating (Gross MHe):

6. Design Electrical Rating (Net MWe):

7. Maximum Dependable Capacity (Gross MWe):

8. Maximum Dependable Capacity (Wet MWe):

9. If Changes Occurred Above Since Last Report, Give Reasons:

10. Power Level To Which Restricted, If Any (Net MMe):

11. Reasons For Restrictions, If Any:

\begin{tabular}{|c|c|c|c|}
\hline & MONTH & YEAR & CUMLATIVE \\
\hline 12. Report Period Hrs & 744.0 & $8,760.0$ & $81,768.0$ \\
\hline 13. Hours Reactor Critical & 744.0 & $7,605.7$ & $65,968.0$ \\
\hline 14. Rx Reserve Shtdwn Hrs & 0.0 & 0.0 & 339.8 \\
\hline 15. Hrs Generator On-Line & 744.0 & $7,501.2$ & $65,154.6$ \\
\hline 16. Unit Reserve Shtdun Hrs & 0.0 & 0.0 & 0.0 \\
\hline 17. Gross Therm Ener (MUH) & $2,637,320.0$ & $25,767,937.0$ & $215,032,350.0$ \\
\hline 18. Gross Elec Ener (MUH) & $915,452.0$ & $8,910,666.0$ & $74,764,215.0$ \\
\hline 19. Net Elec Ener (MUH) & $879,799.0$ & $8,529,002.0$ & $71,374,674.0$ \\
\hline 20. Unit Service Factor & 100.0 & 85.6 & 79.7 \\
\hline 21. Unit Avail Factor & 100.0 & 85.6 & 79.7 \\
\hline 22. Unit Cap factor (MDC Net) & 101.9 & 84.7 & 77.0 \\
\hline 23. Unit Cap Factor (DER Net) & 101.1 & 83.2 & 74.6 \\
\hline 24. Unit Forced Outage Rate & 0.0 & 0.8 & 4.2 \\
\hline 25. Forced Outage Hours & 0.0 & 59.2 & $2,865.0$ \\
\hline
\end{tabular}

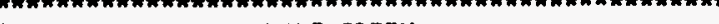 * NOLF CREEK}

WOLF CREEK

AVERAGE DAILY POWER LEVEL (Net MWe)

\begin{tabular}{ll} 
DAY & PONER \\
\hline 1 & 1183 \\
\hline 2 & 1168 \\
\hline 3 & 1180 \\
\hline 4 & 1188 \\
\hline 5 & 1186 \\
\hline 6 & 1168 \\
\hline 7 & 1177 \\
\hline 8 & 1185 \\
\hline 9 & 1182 \\
\hline 10 & 1186 \\
\hline 11 & 1186 \\
\hline 12 & 1186 \\
\hline 13 & 1185 \\
\hline 14 & 1185 \\
\hline 15 & 1184 \\
\hline
\end{tabular}

\begin{tabular}{ll} 
DAY & PONER \\
\hline 16 & 1179 \\
\hline 17 & 1186 \\
\hline 18 & 1186 \\
\hline 19 & 1185 \\
\hline 20 & 1185 \\
\hline 21 & 1185 \\
\hline 22 & 1170 \\
\hline 23 & 1182 \\
\hline 24 & 1186 \\
\hline 25 & 1186 \\
\hline 26 & 1186 \\
\hline 27 & 1186 \\
\hline 28 & 1186 \\
\hline 29 & 1186 \\
\hline 30 & 1174 \\
\hline 31 & 1185 \\
\hline
\end{tabular}

26. Shutdowns Scheduled Over Next six Months (Type, Date, Duration):

27. If Currently Shutdoun, Estimated Startup Date:

Notes:

YEAR-TO-DATE AND CUMULATIVE UNIT CAPACITY FACTORS (MDC NET) ARE CALCULATED WITH WEIGHTED AVERAGES. 


\begin{tabular}{|c|c|}
\hline TYPE & REASON \\
\hline $\begin{array}{l}\text { F: Forced } \\
\text { S: Scheduled }\end{array}$ & $\begin{array}{l}\text { A-Equipment Failure } \\
\text { B-Maintenance or Test } \\
\text { C-Refuel ing } \\
\text { D-Regulatory Restriction } \\
\text { E-Operator Training \& } \\
\text { License Examination } \\
\text { F-Administrative } \\
\text { G-Operat ional Error } \\
\text { H-Other }\end{array}$ \\
\hline
\end{tabular}

\begin{tabular}{l} 
METHCD \\
\hline 1-Manual \\
2-Manual Scram \\
3-Auto Scram \\
4-Continued \\
5-Reduced Load \\
9-0ther
\end{tabular}

SYSTEM

IEEE Standard 805-1984 and/or NUREG-0161 Exhibit $F$
COMPONENT

IEEE Standard

803A- 1983 and/or

NUREG-0161 Exhibit H

F-Adninistrative

H-Other 

.

\section{FACILITY DESCRIPTION}

\section{LOCATION}

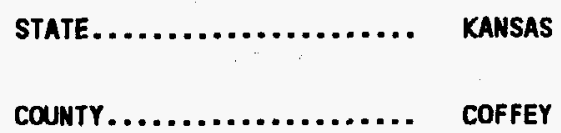

\section{DIST AND DIRECTION FROM}

NEAREST POPULATION CTR..... 3.5 MI NE OF BURLINGTON, KS

TYPE OF REACTOR

PUR

DATE INITIAL CRITICALITY...... MAY 22, 1985

DATE INITIAL ELECTRICITY....... JUNE 12, 1985

DATE COMMERCIAL OPERATE........ SEPTEMBER 03, 1985

CONDENSER COOLIMG METHOD...... COOLING LAKE

CONDENSER COOLING WATER....... WOLF CREEK CLHG LAKE

ELECTRIC RELIABILITY

COUNCIL.................... SOUTHUEST POWER POOL

\section{UTILITY \& CONTRACTOR INFORMATION}

UTILITY

LICENSEE.

WOLF CREEK MUCLEAR OPER. CORP.

CORPORATE ADDRESS.

P.0. BOX 411

BURLINGTON, KANSAS 66839

\section{CONTRACTOR}

ARCHITECT/ENGINEER

BECHTEL

NUC STEAM SYS SUPPLIER...... MESTINGHOUSE

CONSTRUCTOR

DAMIEL INTERMATIONAL

TURBINE SUPPLIER

GENERAL ELECTRIC

\section{REGULATORY IMFORMATION}
IE REGION RESPONSIBLE.........
IE RESIDENT INSPECTOR.......... JACOB F. RINGWALD
LICENSING PROJ MANAGER......... WILLIAM D. RECKLEY
DOCKET NUMBER............... 50-482
LICENSE \& DATE ISSUANCE........ NPF 042, JUME 04, 1985 
1. Docket: 50-295

OPERATING STATUS

2. Reporting Period: DECEMBER 1994

Outage + On-Line Hrs: 744.0

3. Utility Contact: J. CYGAN (708) 746-2084 EXT. 3169

4. Licensed Thermal Power (MUt):

5. Nameplate Rating (Gross Mue):

6. Design Electrical Rating (Net MWe):

7. Maximum Dependable Capacity (Gross MWe):

8. Maximum Dependable Capacity (Net MHe):

9. If Changes Occurred Above Since Last Report, Give Reasons:

10. Power Level To Which Restricted, If Any (Net MWe):

11. Reasons For Restrictions, If Any:

\begin{tabular}{|c|c|c|c|}
\hline & MONTH & YEAR & CUMULATIVE \\
\hline 12. Report Period Hrs & 744.0 & $8,760.0$ & $184,104.0$ \\
\hline 13. Hours Reactor Critical & 744.0 & $4,274.0$ & $123,693.7$ \\
\hline 14. Rx Reserve Shtdin Hrs & 0.0 & 0.0 & $2,621.8$ \\
\hline 15. Hrs Generator On-Line & 744.0 & $4,177.4$ & $120,100.5$ \\
\hline 16. Unit Reserve Shtdun Hrs & 0.0 & 0.0 & 0.0 \\
\hline 17. Gross Therm Ener (MUH) & $2,408,344.0$ & $12,998,973.0$ & $351,572,120.0$ \\
\hline 18. Gross Elec Ener (MWH) & $805,660.0$ & 4.319 .772 .0 & $114,179,332.0$ \\
\hline 19. Net Elec Ener (MUH) & $775,812.0$ & $4,147,265.0$ & $108,693,836.0$ \\
\hline 20. Unit Service Factor & 100.0 & 47.7 & 65.2 \\
\hline 21. Unit Avail Factor & 100.0 & 47.7 & 65.2 \\
\hline 22. Unit Cap factor (MDC Net) & 100.3 & 45.5 & 56.8 \\
\hline 23. Unit Cap Factor (DER Net) & 100.3 & 45.5 & 56.8 \\
\hline 24. Unit forced Outage Rate & 0.0 & 35.8 & 16.8 \\
\hline 25. Forced Outage Hours & 0.0 & 2327.7 & $24,277.2$ \\
\hline
\end{tabular}

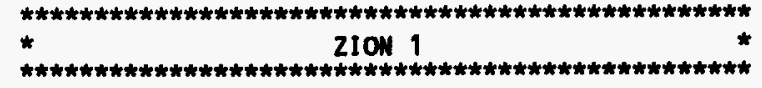

AVERAGe daILY PONER LEVEL (Net MHe)

\begin{tabular}{ll} 
DAY & PONER \\
\hline 1 & 1046 \\
\hline 3 & 1045 \\
\hline 4 & 1046 \\
\hline 5 & 1046 \\
\hline 6 & 1043 \\
\hline 7 & 1045 \\
\hline 8 & 1045 \\
\hline 9 & 1043 \\
\hline 10 & 1044 \\
\hline 11 & 1044 \\
\hline 12 & 1038 \\
\hline 13 & 1041 \\
\hline 14 & 1043 \\
\hline 15 & 1042 \\
\hline
\end{tabular}

\begin{tabular}{ll} 
DAY & PONER \\
\hline 16 & 1044 \\
\hline 17 & 1043 \\
\hline 18 & 1043 \\
\hline 19 & 1043 \\
\hline 20 & 1043 \\
\hline 21 & 1045 \\
\hline 22 & 1045 \\
\hline 23 & 1044 \\
\hline 24 & 1043 \\
\hline 25 & 1043 \\
\hline 26 & 1042 \\
\hline 27 & 1043 \\
\hline 28 & 1042 \\
\hline 29 & 1040 \\
\hline 30 & 1041 \\
\hline 31 & 1026 \\
\hline
\end{tabular}

26. Shutdowns Scheduled Over Next six Months (Type, Date, Duration):

27. If Currently Shutdown, Estimated Startup Date:

Notes: 

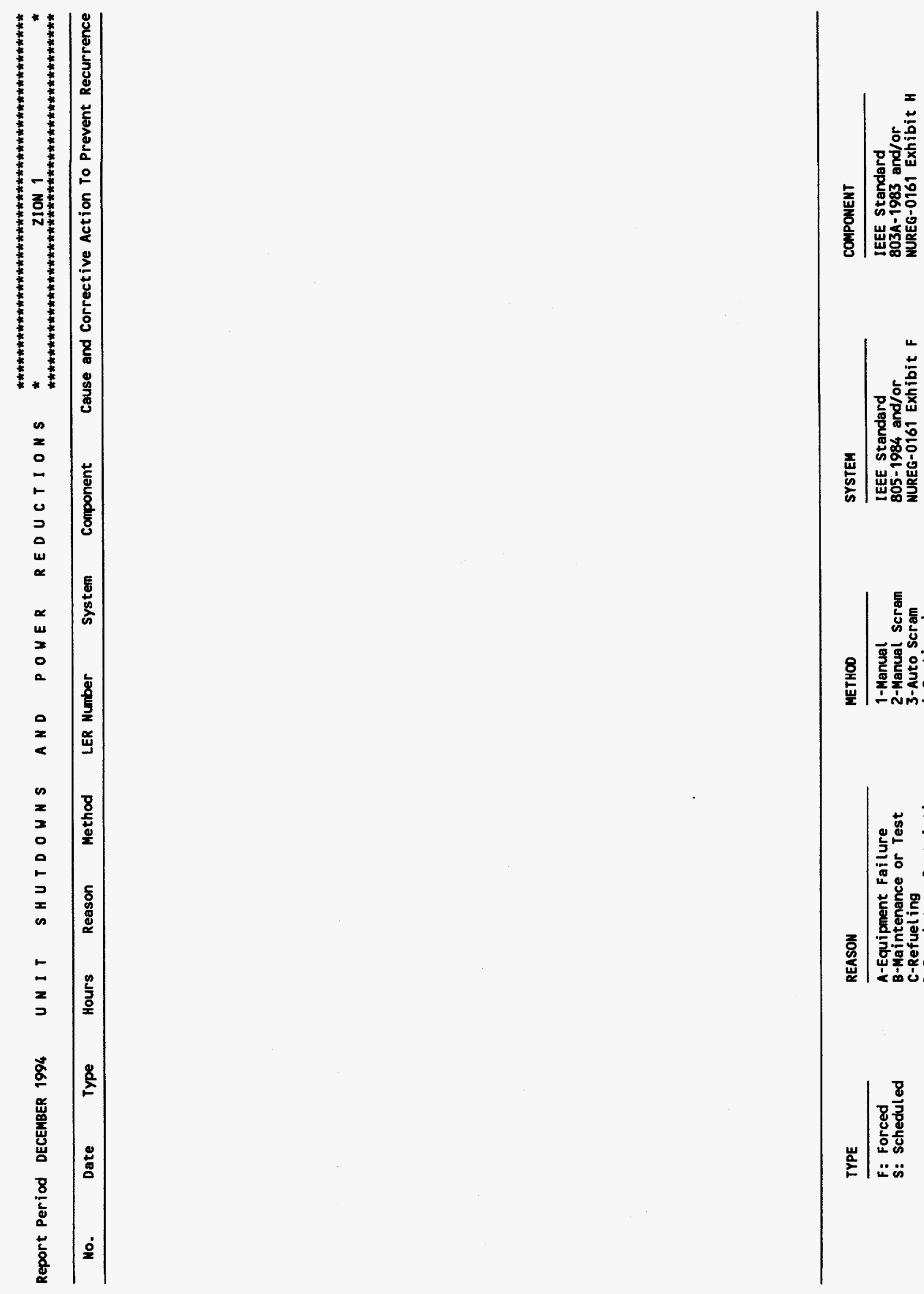

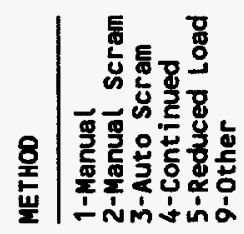

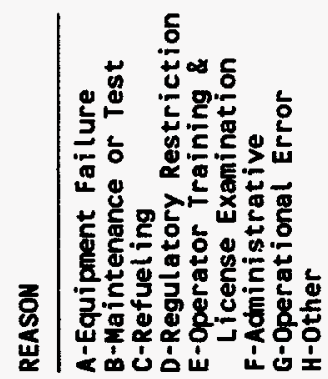

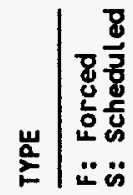




\section{FACILITY DESCRIPTION}

LOCATION

STATE.

COUNTY

ILLINOIS

LAKE

DIST AND DIRECTION FRON

MEAREST POPULATION CTR

40 MI N OF CHICAGO, IL

TYPE OF REACTOR.

PWR

DATE INITIAL CRITICALITY...... JUNE 19, 1973

DATE INITIAL ELECTRICITY...... JUNE 28, 1973

OATE COMmerCIAL OPERATE....... DECEMBER 31, 1973

CONDENSER COOLING METHOD...... ONCE THRU

CONDENSER COOLING WATER....... LAKE MICHIGAN

ELECTRIC RELIABILITY

COUNCIL.................. MID-AMERICA INTERPOOL NETHORK

\section{UTILITY \& CONTRACTOR INFORMATION}

\section{UTILITY}

LICENSEE.

CORPORATE ADDRESS.

COMMONWEALTH EDISON CO.

1400 OPUS PL., OPUS WEST III

SUITE 300

DOWNER'S GROVE, ILLIMOIS 60515

CONTRACTOR

ARCHITECT/ENGINEER.......... SARGENT \& LUMDY

MUC STEAM SYS SUPPLIER...... WESTIMGHOUSE

CONSTRUCTOR.............. COMMOMUEALTH EDISOM

TURBINE SUPPLIER.......... WESTINGHOUSE

\section{REGULATORY INFORMATION}

IE REGION RESPONSIBLE......... 3

IE RESIDENT INSPECTOR......... JAMES ROTON

LICENSING PROJ MANAGER......... CLYDE Y. SHIRAKI

DOCKET MUMBER.............. 50-295

LICENSE \& DATE ISSUANCE........ DPR 039, OCTOBER 19, 1973 
1. Docket: $50-304$

OPERATING STATUS

2. Reporting Period: DECEMBER 1994

Outage + On-Line Hrs: $\mathbf{7 4 4 . 0}$

3. Utility Contact: J. CYGAN (708) 746-2084 EXT. 3169

4. Licensed Thermal Power (mit):

5. Nameplate Rating (Gross MWe):

6. Design Electrical Rating (Net MWe):

7. Maximum Dependable Capacity (Gross MHe):

8. Maximum Dependable Capacity (Net MWe):

9. If Changes Occurred Above since Last Report, Give Reasons:

10. Power Level To Which Restricted, If Any (Net MWe):

11. Reasons For Restrictions, If Any:

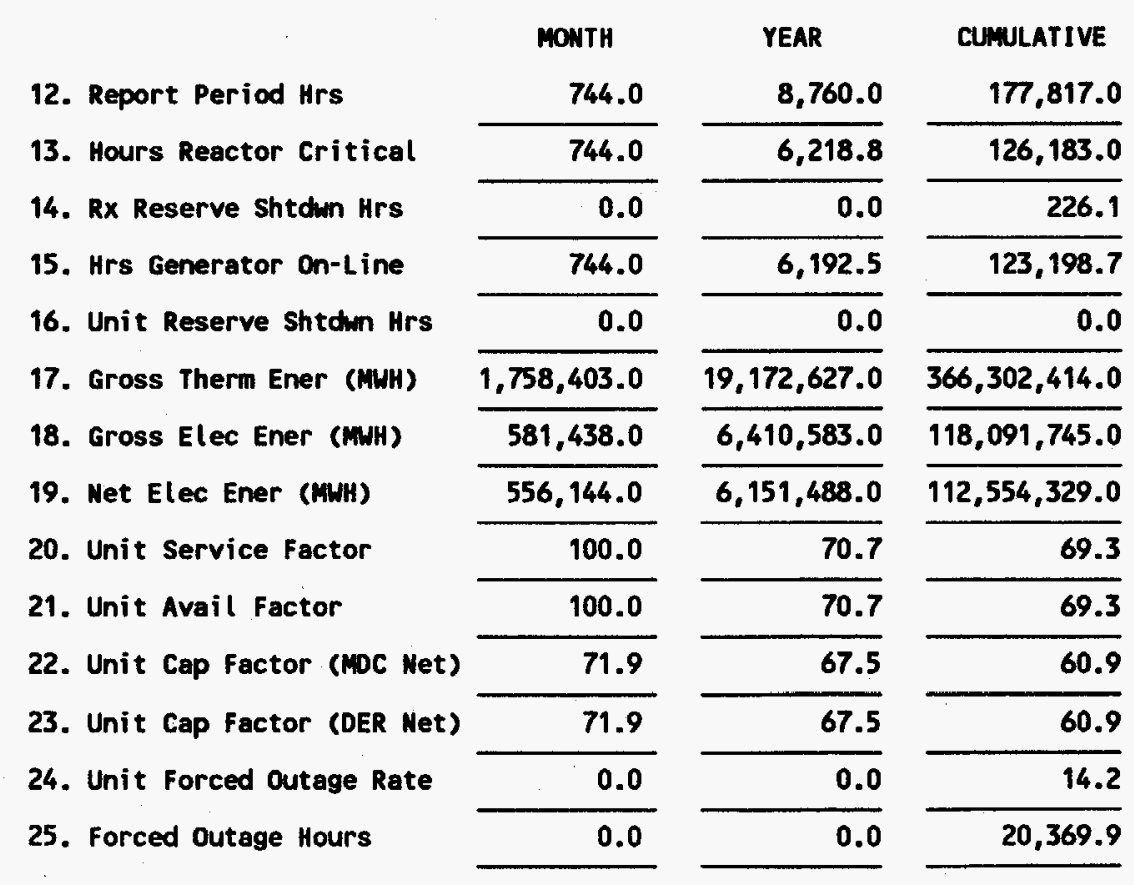

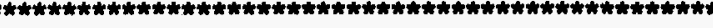

* ZION 2 *

AVERAGE DAILY PONER LEVEL (Net MNe)

\begin{tabular}{ll} 
DAY & PONER \\
\hline 1 & 898 \\
\hline 2 & 888 \\
\hline 3 & 878 \\
\hline 4 & 868 \\
\hline 5 & 851 \\
\hline 6 & 841 \\
\hline 7 & 830 \\
\hline 8 & 816 \\
\hline 9 & 810 \\
\hline 10 & 799 \\
\hline 11 & 787 \\
\hline 12 & 782 \\
\hline 13 & 772 \\
\hline 14 & 764 \\
\hline 15 & 750 \\
\hline
\end{tabular}

\begin{tabular}{ll} 
DAY & PONER \\
\hline 16 & 740 \\
\hline 17 & 731 \\
\hline 18 & 721 \\
\hline 19 & 713 \\
\hline 20 & 704 \\
\hline 21 & 699 \\
\hline 22 & 688 \\
\hline 23 & 682 \\
\hline 24 & 673 \\
\hline 25 & 668 \\
\hline 26 & 657 \\
\hline 27 & 647 \\
\hline 28 & 638 \\
\hline 29 & 637 \\
\hline 30 & 626 \\
\hline 31 & 616 \\
\hline
\end{tabular}

26. Shutdowns Scheduled Over Mext Six Months (Type, Date, Duration): REFUELING OUTAGE, JANUARY 6, 1995, 76 DAYS.

27. If Currently Shutdown, Estimated Startup Date: Notes: 
TYPE

F: Forced

S: Scheduled

\section{REASON}

A-Equipment Failure B-Maintenance or Test C-Refuel ing

D-Regulatory Restriction

-Operator Training \&

License Examination

F-Acministrative

G-Operational Error

-other

\section{METHOD}

1-Manua

2-Manual Scram

3-Auto Scram

4-Cont inued

5-Reduced Load

9-other

\section{SYSTEM}

IEEE Standard

NUREG-0161 Exhibit $F$

\section{COMPONENT}

IEEE Standard

803A-1983 and/or
MUREG-0161 Exhibit $h$ 
FACILITY DESCRIPTION

LOCATION

STATE.

COUNTY.

ILLIMOIS

LAKE

\section{DIST AND DIRECTION FROM}

NEAREST POPULATION CTR..... 40 MI N OF CHICAGO, IL

TYPE OF REACTOR.

DATE INITIAL CRITICALITY...... DECEMBER 24, 1973

DATE INITIAL ELECTRICITY...... DeCEMBER 26, 1973

date commercial operate...... September 17,1974

CONDENSER COOLING METHOD...... ONCE THRU

CONDENSER COOLIMG MATER....... LAKE MICHIGAN

ELECTRIC RELIABILITY

COUNCIL................... MID-AMERICA INTERPOOL NETWORK
UTILITY \& CONTRACTOR INFORMATION

\section{UTILITY}

LICENSEE................. COMMONMEALTH EDISON CO.

CORPORATE ADDRESS

1400 OPUS PL., OPUS VEST III

SUITE 300

DOWNER'S GROVE, ILLINOIS 60515

\section{CONTRACTOR}

ARCHITECT/ENGINEER......... SARGENT \& LUNDY

NUC STEAM SYS SUPPLIER...... MESTIMGHOUSE

CONSTRUCTOR............... COMHONUEALTH EDISON

TURBINE SUPPLIER........... WESTINGHOUSE

\section{REGULATORY INFORMATION}

IE REGION RESPONSIBLE........ 3

IE RESIDENT INSPECTOR......... JAMES ROTON

LICENSING PROJ MANAGER........ CLYDE Y. SHIRAKI

DOCKET NUMBER............. 50-304

LICENSE \& DATE ISSUANCE........ DPR 048, NOVEMBER 14, 1973 


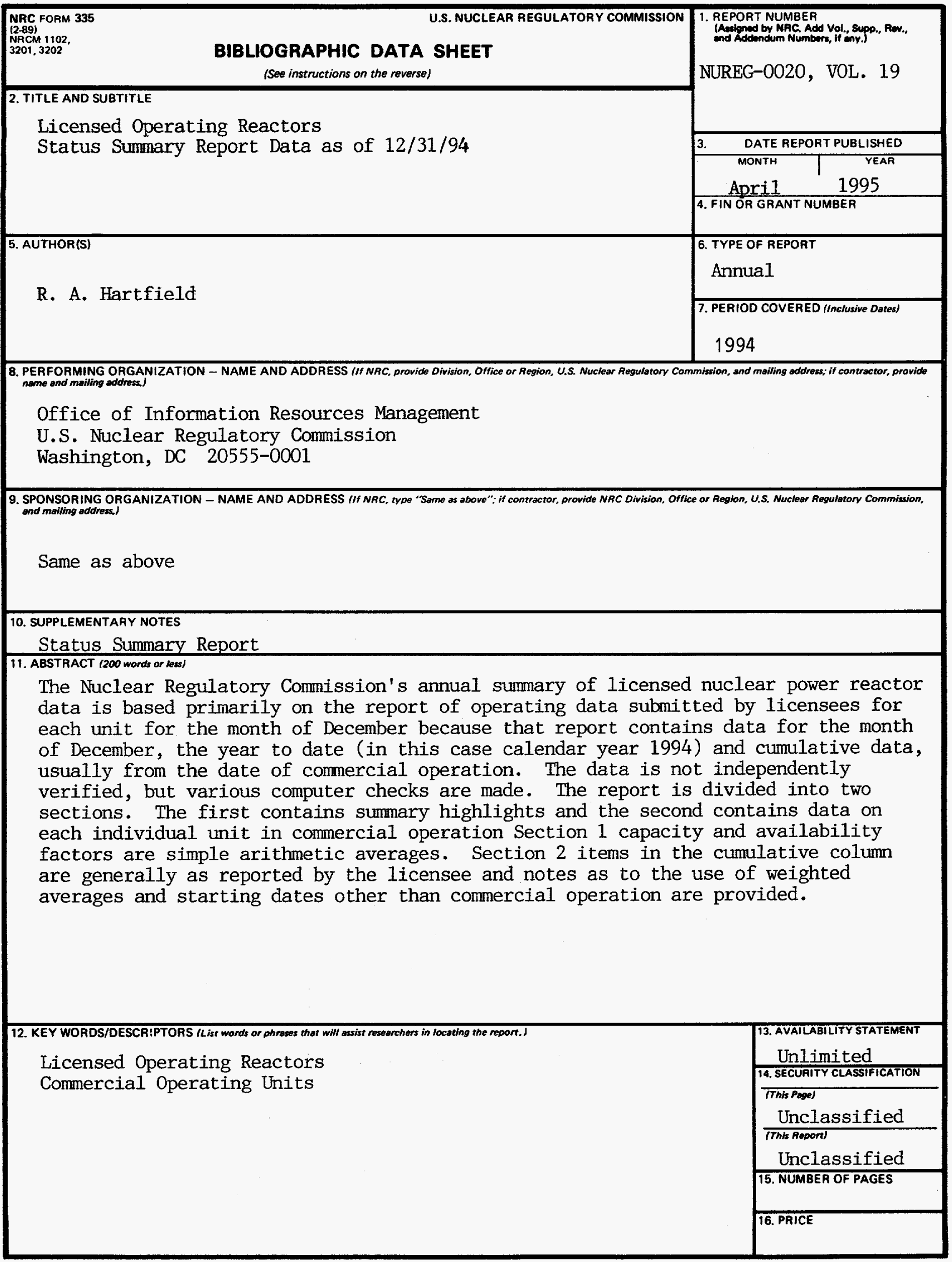

